\title{
IMPUTAÇÃO DE DADOS EM PAINÉIS PARA POPULAÇÕES FINITAS
}

\author{
Lúcia Pereira Barroso
}

TESE DEFENDIDA

NO

INSTITUTO DE MATEMÁTICA E ESTATÍSTICA

DA

UNIVERSIDADE DE SÃO PAULO

PARA OBTENÇÃO DO GRAU DE DOUTOR

EM

ESTATÍSTICA

Área de concentração: Estatística
Orientador: Prof. Dr. Wilton de Oliveira Bussab

Durante a elaboração deste trabalho, a autora recebeu apoio financeiro do CNPq

- São Paulo, abril de 1995 - 


\section{IMPUTAÇÃO DE DADOS EM PAINÉIS PARA POPULAÇÕES FINITAS}

Este exemplar corresponde à redação final da tese devidamente corrigida e defendida

por Lúcia Pereira Barroso e aprovada pela Comissão Julgadora

São Paulo, 05 de abril de 1995

\section{Banca Examinadora:}

- Prof. Dr. Wilton de Oliveira Bussab (Orientador) - IME-USP

- Prof. Dr. Heleno Bolfarine - IME-USP

- Prof. Dr. Josemar Rodrigues - ICMSC-USP

- Prof a. Dr a Édina S. Miazaki - UnB

- Prof. Dr. José Francisco Soares - UFMG 
$\grave{A}$

Luciana, Fernanda, Renata e Victoria 


\section{AGRADECIMENTOS}

O término desta tese acabou por me fazer desfrutar de sensações opostas: o final e o começo. Por um lado, tive a satisfação de ter concluído um trabalho, que é o resultado de alguns anos de pesquisa, durante os quais conheci sentimentos como paciência, persistência, cansaço, insegurança, segurança, apoio, incentivo e outros que acabaram culminando com a alegria da descoberta. Por outro lado, tenho a sensação de que isto é só o começo e que vou poder usar o aprendizado e amadurecimento profissional que adquiri nesse período.

Esta tese certamente não seria concluída sem a colaboração de algumas pessoas, a quem quero fazer o meu mais sincero agradecimento. Agradeço ao Professor Wilton de Oliveira Bussab, pela sugestão do tema e idéias e pela dedicação e paciência com que me orientou. A convivência com ele, além de me trazer um grande amigo, muito me ensinou e eu sempre me pergunto se um dia vou poder ter tanto conhecimento quanto ele. Agradeço também ao Professor Martin Knott pela paciência com que discutiu meu trabalho durante minha visita à London School of Economics and Political Science da University of London. Gostaria ainda de agradecer:

- aos Professores Heleno Bolfarine e Dalton Francisco de Andrade pela atenção, sugestões e esclarecimentos na parte final deste trabalho;

- aos amigos Pedro Luis do Nascimento Silva, Denise Britz do Nascimento Silva e Denise A parecida Botter pelo apoio, amizade e por termos partilhado tantos momentos na Inglaterra;

- aos amigos do IME-USP, pelo carinho, solidariedade e incentivo;

- ao CNPq pelo suporte financeiro durante minha estada em Londres.

Finalmente, gostaria de agradecer à minha família, que acompanhou, incentivou e torceu pelo sucesso desta tese. 


\section{RESUMO}

Um problema bastante comum em levantamentos amostrais é a falta de algumas das respostas. Uma das maneiras de tratar esse problema é a imputação, que consiste em prever as observações perdidas, completando o conjunto de dados para depois analisá-lo como se fosse completo. Estudamos, neste trabalho, a falta de respostas em conjuntos de dados longitudinais, considerando como amostra o painel sorteado de populações finitas.O principal objetivo desta tese é a obtenção do previsor linear não-viesado, de erro quadrático médio mínimo, conhecido na literatura como BLUP (Best Linear Unbiased Predictor), para os efeitos fixos e aleatórios e para as observações ausentes, sob o modelo linear misto. Consideramos o tempo como um fator fixo e os indivíduos como um fator aleatório, relaxando as suposições usuais de normalidade e independência. Estudamos os casos univariado e multivariado sob a suposição de variâncias conhecidas e desconhecidas, considerando os seguintes métodos de estimação: análise de variância, estimação de máxima verossimilhança restrita e estimação quadrática não-viesada de norma mínima. Apresentamos o erro quadrático médio dos previsores e a variância dos estimadores das componentes da variância. Fizemos ainda uma conexão com o caso de populações infinitas. Como resultado, obtivemos fórmulas interpretáveis e de fácil aplicação pois requerem apenas cálculos simples com matrizes. 


\section{ABSTRACT}

A common problem in sampling surveys is the occurrence of nonresponses. One of the ways to handle this problem is by means of imputation, which consists of predicting the missing observations to complete the data set which will be later analised. In this thesis we study the lack of responses in longitudinal data sets, considering as our sample a panel selected from finites populations. Our main objective is to obtain the best linear unbiased predictor (BLUP) to the fixed and random effects and the missing observations, under a mixed linear model. We consider time as a fixed effect and individuals as a random effect, relaxing the usual assumptions of normality and independence. We study the univariate and multivariate cases under the assumption of known and unknown variances, considering the following estimation methods: analysis of variance, restricted estimation of maximum likelihood (REML) and minimum norm quadratic unbiased estimation (MINQUE). We present the mean square error of the predictors and the variance of the variance components estimators. We have also made a conection with the case of infinite population. We have obtained as result formulas which can easily be interpreted and applied since they require simple matrix calculations. 


\section{ÍNDICE}

1. CAPÍtUlo 1: intRoduÇÃO . . . . . . . . . . . . . . . 1

2. CAPÍtUlO 2: BLUP (BEST LINEAR UNBIASED PREDICTOR) PARA O MODELO MISTO

2.1. Introdução . . . . . . . . . . . . . . . . . . . 15

2.2. Estimação e previsão com variâncias conhecidas . . . . . . . . . . . 25

2.3. Simplificação dos previsores . . . . . . . . . . . . . . . . . . 37

2.4. Erro quadrático médio dos previsores . . . . . . . . . . . . . 41

3. CAPÍTULO 3: CASO UNIVARIADO

3.1. Introdução . . . . . . . . . . . . . . . . . . . . . . 43

3.2. Caso 1: uma observação perdida no último instante . . . . . . . . . . 49

3.2.1. Conexão com o caso de populações infinitas . . . . . . . . 53

3.3. Caso 2: mais de uma observação perdida em um só instante . . . . . . 56

3.4. Caso 3: mais de uma observação perdida em qualquer instante . . . . . 58

3.5. Uma ilustração numérica . . . . . . . . . . . . . . . . . . 70

4. CAPÍTUlO 4: CASO MULTIVARIADO

4.1. Introdução . . . . . . . . . . . . . . . . . 73

4.2. Caso 1: uma observação perdida no último instante . . . . . . . . . 79

4.3. Caso $2: m$ observações perdidas em qualquer instante . . . . . . . . . 84

4.4. Uma ilustração numérica . . . . . . . . . . . . . . . . . . 87

5. CAPÍTULO 5: O CASO DE VARIÂNCIAS DESCONHECIDAS

5.1. Introdução 
5.2. Caso univariado . . . . . . . . . . . . . . . . . . . 94

5.2.1. O método de análise de variância - ANOVA . . . . . . . . . 94

5.2.2. O método de estimação de máxima verossimilhança restrita REML . . . . . . . . . . . . . . . 97

5.2.3. O método de estimação quadrática não-viesada de norma mínima - MINQUE . . . . . . . . . . . . . 101

5.2.4. Variância dos estimadores . . . . . . . . . . . . 107

5.3. Caso multivariado . . . . . . . . . . . . . . . . . . . . . . 110

5.4. Uma ilustração numérica . . . . . . . . . . . . . . . . . . . . 114

6. CAPÍTUlO 6: ILUSTRAÇÕES NUMÉRICAS

6.1. Introdução . . . . . . . . . . . . . . . . . . . 115

6.2. Exemplo 1: total de pessoal ocupado e horas trabalhadas na produção . . . . . . . . . . . . . . . . . . . . 115

6.3. Exemplo 2: total de salários nominais . . . . . . . . . . . . . 118

6.4. Exemplo 3: os dados de Potthoff e Roy . . . . . . . . . . . . . . . 119

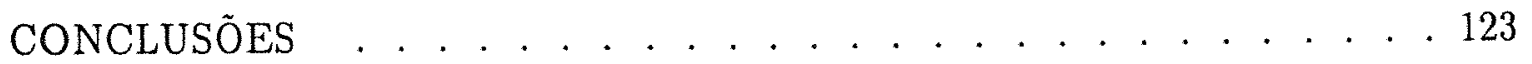

APÊNDICE A: RESULTADOS UTILIZADOS . . . . . . . . . . . . . . 126

APÊNDICE B: CASO UNIVARIADO . . . . . . . . . . . . . 130

APÊNDICE C: CASO MULTIVARIADO . . . . . . . . . . . . 146

APÊNDICE D: ESTIMAÇÃO DA VARIÂNCIA . . . . . . . . . . . . 155

REFERENCIAS . . . . . . . . . . . . . . . . 163 


\section{NOTAÇÃO}

$\mathrm{y}=\mathrm{X} \alpha+\mathrm{Zb}+\mathrm{e}$

onde

y: vetor de $d$ variáveis aleatórias observáveis;

$\alpha$ : vetor de $p$ parâmetros fixos desconhecidos (efeitos fixos);

b: vetor de $q$ variáveis aleatórias não observáveis (efeitos aleatórios);

$\mathrm{X}$ e Z: matrizes conhecidas;

e: vetor de variáveis aleatórias (erros)

$\mathbf{y}_{\mathbf{o}}:$ parte observada de $\mathrm{y}$

$\mathbf{y}_{\mathbf{a}}:$ parte ausente de $\mathrm{y}$

$\mathrm{X}_{o}$ : parte observada de $\mathrm{X}$

$\mathrm{X}_{a}$ : parte ausente de $\mathrm{X}$

$\mathbf{Z}_{o}$ : parte observada de $\mathbf{Z}$

$\mathbf{Z}_{a}$ : parte ausente de $\mathbf{Z}$

E: matriz indicadora dos dados ausentes

F: matriz indicadora dos dados presentes

$\mathrm{V}=\operatorname{Var}(\mathrm{y})$

$1_{n}$; vetor coluna de 1's de dimensão $n$;

$\mathrm{I}_{n}$ : matriz identidade de dimensão $n$;

$\mathbf{J}_{T}$ : matriz quadrada de 1's de dimensão $T$;

Q: produto de Kronecker

$N$ : tamanho da população 
$n:$ tamanho da amostra

$T$ : número de instantes observados

$m$ : número de observaçôes perdidas

\section{Caso Univariado}

$$
\begin{aligned}
& y_{t i}=\mu+\alpha_{t}+b_{i}+e_{t i} \quad t=1,2, \ldots, T \\
& i=1,2, \ldots, n \\
& \sigma^{2}=\operatorname{Var}\left(e_{t i}\right) \quad S^{2}=\frac{1}{N-1} \sum_{i=1}^{N} b_{i}^{2} \quad K=\frac{S^{2}}{\sigma^{2}} \\
& \mathrm{~V}=\operatorname{Var}(\mathrm{y})=\mathrm{W} \sigma^{2} \\
& \mathbf{W}=\left(\mathbf{I}_{n} \otimes \mathbf{I}_{T}\right)+K\left(\mathbf{I}_{n} \otimes \mathbf{J}_{T}\right)-\frac{K}{N}\left(\mathbf{J}_{n} \otimes \mathbf{J}_{T}\right) \\
& \mathbf{W}^{-1}=\left(\mathbf{I}_{n} \otimes \mathbf{I}_{T}\right)-a\left(\mathbf{I}_{n} \otimes \mathbf{J}_{T}\right)+b\left(\mathbf{J}_{n} \otimes \mathbf{J}_{T}\right) \\
& \mathbf{Q}=\frac{1}{n}\left(\mathbf{J}_{n} \otimes \mathrm{I}_{T}\right)+a\left(\mathrm{I}_{n} \otimes \mathbf{J}_{T}\right)-\frac{a}{n}\left(\mathbf{J}_{n} \otimes \mathbf{J}_{T}\right) \\
& \operatorname{com} a=\frac{K}{(T K+1)} \quad, \quad b=\frac{K}{N(T K+1)(T K(1-f)+1)} \quad \text { e } \quad f=\frac{n}{N} \text { (fração amostral). }
\end{aligned}
$$

\section{Caso Multivariado}

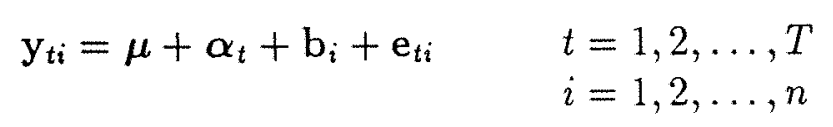

$p$ : número de variáveis

$$
\Sigma=\operatorname{Var}\left(\mathrm{e}_{t i}\right) \quad S_{j j^{\prime}}=\frac{1}{N-1} \sum_{i=1}^{N} b_{j i} b_{j^{\prime} i} \quad j, j^{\prime}=1,2, \ldots, p
$$

S: matriz $\left(S_{i j}\right)$

$$
\mathrm{V}=\left(\mathrm{I}_{n} \otimes \mathrm{I}_{T} \otimes \boldsymbol{\Sigma}\right)+\left(\mathrm{I}_{n} \otimes \mathrm{J}_{T} \otimes \mathrm{S}\right)-\frac{1}{N}\left(\mathbf{J}_{n} \otimes \mathbf{J}_{T} \otimes \mathrm{S}\right)
$$$$
\mathrm{V}^{-1}=\left(\mathrm{I}_{n} \otimes \mathrm{I}_{T} \otimes \mathbf{\Sigma}^{-1}\right)-\left(\mathrm{I}_{n} \otimes \mathrm{J}_{T} \otimes \mathrm{H}\right)+\left(\mathrm{J}_{n} \otimes \mathrm{J}_{T} \otimes \mathrm{G}\right)
$$$$
\mathrm{Q}=\left(\mathrm{I}_{n} \otimes \mathrm{I}_{T} \otimes \mathrm{I}_{p}\right)+\left(\frac{1}{n} \mathrm{~J}_{n}-\mathrm{I}_{n}\right) \otimes\left(\mathrm{I}_{T} \otimes \Sigma^{-1}-\mathrm{J}_{T} \otimes \mathrm{H}\right)
$$

onde

$$
\begin{aligned}
& \mathbf{H}=(T \mathbf{S}+\Sigma)^{-1} \mathbf{S} \Sigma^{-1} \\
& \mathbf{G}=\frac{1}{N}(T(1-f) \mathbf{S}+\Sigma)^{-1} \Sigma(T S+\Sigma)^{-1} \mathbf{S} \Sigma^{-1}
\end{aligned}
$$




\section{CAPÍTULO 1}

\section{INTRODUÇÃO}

Na maioria dos levantamentos amostrais é comum a ocorrência de dois tipos de erros: os erros amostrais e os erros não-amostrais. Os primeiros são devidos ao fato de que somente uma parte da população está sendo investigada. Mas, mesmo que o estudo seja feito através de censo, com a observação de toda a população, e nesse caso não teríamos o erro amostral, ainda estaríamos sujeitos a erro: ao erro não-amostral.

Esse tipo de erro pode ser devido a diversas causas, como por exemplo questões mal formuladas em um questionário, falhas de planejamento, falta de treinamento ou mesmo má vontade dos entrevistadores e/ou entrevistados, respostas falsas por parte dos respondentes, erros de transcrição ou digitação, etc.

Quando esses erros são detectados, na impossibilidade de corrigí-los, é comum descar- 
tar tais informações, o que gera lacunas ou erros de não-resposta no conjunto de dados.

Entretanto, não somente o descarte de informações pode causar essas falhas. Às vezes o próprio respondente se recusa ou está impossibilitado de fornecer os dados desejados.

A não-resposta pode ser total ou parcial: total, quando todas as informações são perdidas e parcial, quando são perdidas somente uma parte delas. Nesse caso, a nãoresposta também é conhecida como não-resposta de ítem. Little e Rubin (1983) fazem um histórico sobre o problema de dados incompletos.

Segundo Rubin (1976) e Laird (1988), se o mecanismo de não-resposta não depende da variável de interesse, dizemos que os dados são perdidos por um processo totalmente aleatório (MCAR - missing completely at random). Quando a probabilidade de nãoresposta depende dos dados presentes mas não dos ausentes, os dados são ditos perdidos por um processo aleatório (MAR - missing at random) e o mecanismo de não-resposta ignorável. O termo ignorável foi usado para indicar que não é necessário especificar um modelo de não-resposta. É importante salientar que é o processo de não-resposta que pode ser ignorado e não os dados ausentes ou as unidades com dados ausentes.

O processo de não-resposta pode ainda ser não-ignorável, quando a falta dos dados não é aleatória (NMAR - not missing at random). Esse é o caso em que a probabilidade de não-resposta depende dos dados ausentes. Esse tipo de não-resposta pode ocorrer, por exemplo, quando a variável de interesse é renda. A prática tem mostrado que a perda de informações é maior entre as pessoas de maior renda e assim, a não-resposta depende dos dados ausentes.

Através do exame dos dados não é possível detectar se a não-resposta é ignorável ou não. Neste trabalho, estamos considerando o caso em que o processo de não-resposta é ignorável. 
O tratamento de dados incompletos pode ser classificado, segundo Little e Rubin (1987), nas seguintes categorias:

- eliminação das unidades parcialmente observadas: nesse método as unidades com erro de não-resposta parcial são descartadas e a análise é feita com base nos dados completos. Esse pode ser um método satisfatório desde que seja válida a suposição de que a falta de resposta seja completamente aleatória, não dependendo da variável sujeita a não-resposta. Fora isso, pode-se ainda obter bons resultados se o número de não-respostas, e portanto o descarte de observações, for pequeno.

- procedimentos de ponderação: este procedimento é geralmente aplicado ao caso de não-resposta total, como sugerido por Kalton e Kasprzyk (1982) e Duncan e Kalton (1987). As unidades amostrais são reponderadas para levar em consideração a não-resposta e o planejamento amostral.

- procedimentos de imputação: outra maneira de tratar os dados incompletos é a utilização de métodos de imputação, principalmente para o caso de não-resposta parcial, para completar os dados ausentes ou substituir os dados rejeitados. Essa técnica consiste em prever os valores perdidos e então analisar os dados completados. Embora com muitas críticas (vide Bannister (1980)), os procedimentos de imputação também podem ser aplicados ao tratamento da não-resposta total.

Wang, Sedransk e Jinn (1992) assumem que o mecanismo de perda não pode ser considerado ignorável e apresentam uma interessante tabela sobre os tipos de análise de dados compreendendo: utilização somente dos dados observados; utilização dos dados observados mais imputados como se fossem observados; mesmo procedimento anterior 
para os parâmetros de locação com melhoria dos estimadores da variância e imputação múltipla (veja página 6 deste trabalho). Além disso, dão referências classificadas segundo essa tabela.

Neste trabalho nos deteremos na categoria dos procedimentos de imputação. A bibliografia sobre o assunto é ainda bastante restrita, principalmente se a abordagem for a clássica. Nas referências deste estudo, estamos assinalando com $(*)$ os trabalhos que tratam de imputação.

Por método de imputação entende-se todo procedimento de previsão de valores individuais, isto é, por qualquer método que atribua um valor específico a cada dado ausente ou rejeitado. Por exemplo, se $y_{i}$ indica a observação perdida da $i$-ésima unidade amostral, em um método de imputação, $y_{i}$ poderia ser descrito como função de variáveis auxiliares $\mathbf{z}$ como $y_{i}=f(\mathbf{z})+e_{i}$, onde $e_{i}$ é um resíduo aleatório.

Muitas vezes a imputação de valores é feita sem o objetivo específico de estimar qualquer parâmetro. A idéia é completar os dados para futuros estudos e diferentes finalidades. Em estudos censitários, a imputação, além de uma possível técnica, é uma necessidade.

A imputação de valores é atualmente um ramo de grande destaque e muitos esforços têm sido feitos para a obtenção de critérios adequados. Essa técnica, bastante utilizada na área de Agricultura, Melhoramento Animal, Economia e Dados Censitários, iniciouse na área de Agricultura e foi proposta por Allan e Wishart (1930), que introduziram fórmulas para imputar valores para uma única observação perdida em planejamentos de blocos aleatorizados e quadrados latinos.

Mais tarde, também na área de Agricultura, Yates (1933) estudou o caso da falta de várias observações e mostrou que o previsor por ele proposto pode ser obtido através da minimização da soma de quadrados de resíduos. 
Vários pesquisadores têm se dedicado ao assunto. Silva (1989) descreveu a metodologia proposta por Little e Smith (1987) e apresentou rotinas computacionais codificadas na linguagem do SAS - Statistical Analysis Systems, desenvolvidas para a aplicação da metodologia. Albieri (1989), com base no trabalho de Kalton e Kasprzyk (1982), apresentou vários métodos de imputação, dando os estimadores da média com seus respectivos vícios e variâncias.

Nesses trabalhos, os métodos de imputação foram classificados como determinísticos ou estocásticos, dependendo se os resíduos assumem sempre o valor zero ou não.

No caso dos métodos estocásticos, os resíduos podem ser escolhidos de uma suposta distribuição de probabilidade ou sorteados de um "banco de resíduos" construído da distribuição empírica dos dados completos.

Dos trabalhos citados acima pode-se extrair algumas comparações e limitações dos procedimentos determinísticos e estocásticos. Ambos reduzem o vício de estimativas quando se está interessado em estimar a média ou o total da população. Os métodos determinísticos são mais precisos, mas distorcem a distribuição original, tendendo a subestimar a variância da amostra. Os estocásticos, embora com maior erro padrão do estimador, preservam a variabilidade dos dados completos. Em ambos, quanto maior a taxa de não-resposta, piores os resultados obtidos após a imputação.

Bartlett (1937) sugeriu, para a previsão de valores ausentes, o uso de variáveis "dummy" em análise de covariância. Essa técnica, entretanto, tem sido considerada computacionalmente trabalhosa, mas John e Prescott (1975) tentaram enfatizar que ela pode ser preferivel a métodos iterativos.

Alguns autores trabalharam com o modelo linear de efeitos fixos ou o modelo de regressão $\mathrm{y}=\mathrm{X} \beta+\mathrm{e}$ onde $\mathrm{X}$ é fixado e conhecido. Todos eles supuseram independência 
entre os individuos. Nem todos usaram a técnica de imputação. Wilkinson (1958a, 1958b) considerou o estimador de mínimos quadrados e mais tarde Jarret (1978) mostrou que esse método é mais viável do que alguns métodos iterativos encontrados na literatura.

Basilevsky, Sabourin, Hum e Anderson (1985) deram uma relação de estimadores com dados incompletos, detalhadas anteriormente por Anderson, Basilevsky e Hum (1983), e fizeram comparações entre eles usando simulação estruturada e três diferentes critérios de regressão: $R^{2}$, quadrados médios e erro quadrático médio. Ali e Abu-Salih (1988) obtiveram um estimador do tipo James-Stein (1961) para o vetor de observações perdidas num modelo de regressão linear, supondo normalidade dos erros. Subramani e Ponnuswamy (1989) consideraram o planejamento por blocos aleatorizados e quadrado latino e compararam seus resultados com aqueles obtidos por Yates (1933).

Outros que também utilizaram o modelo com fatores fixos foram Hunt e Triggs (1989), que propuseram um método de imputação iterativo, Cooper, De Leeuw e Sogomonian (1991) e Singh e Pratap (1989), que obtiveram estimadores de mínimos quadrados para os efeitos fixos e as observações ausentes. O método proposto pelos últimos é não iterativo e depende da repetição de uma certa sub-rotina e da inversão de uma matriz de ordem igual ao número de observações perdidas.

Ainda sob o modelo de efeitos fixos, Subramani (1992) apresentou um método alternativo à ANOVA (analysis of variance), denominado ANON (analysis of means). Já Ibrahim (1990) considerou a classe de modelos lineares generalizados com covariadas discretas e estudou o caso em que as covariadas são parcialmente ausentes, usando o algoritmo EM (ver Dempster, Laird e Rubin (1977)).

Outra maneira de tratar a não-resposta é a imputação múltipla sugerida por Rubin (1987). Nesse procedimento, a imputação é repetida várias vezes, com a possibilidade 
da utilização de diferentes métodos. Os vários conjuntos de dados completados podem então ser analisados e essas análises comparadas. Uma crítica a esse método é a maior quantidade de trabalho requerida, a maior complexidade e a necessidade de mais espaço para arquivar os dados. O estudo desse critério não faz parte do escopo deste trabalho.

Uma outra situação a estudar é aquela em que aparecem observações perdidas em dados longitudinais, ou seja, alguns pesquisadores consideraram medidas feitas em vários instantes do tempo. Nesse caso, costuma-se utilizar um dos três tipos de amostras: amostra independente, painel fixo e painel rotativo, cujas definições damos abaixo:

- amostra independente: as amostras são selecionadas da população em várias ocasiões no tempo, independentemente umas das outras;

- painel fixo: uma amostra é selecionada num primeiro instante e depois os dados são coletados, dessa mesma amostra, para várias ocasiões. Esse tipo de amostra é também conhecido simplesmente como painel;

- painel rotativo: uma amostra é selecionada em um determinado instante e, na ocasião seguinte, uma parte dessa amostra é mantida e o restante é substituído por novos elementos, através de 'novo sorteio.

Cada um desses tipos de planos amostrais apresentam vantagens e desvantagens e algumas delas, tiradas de Cochran (1977) e de Duncan e Kalton (1987), serão apresentadas a seguir.

Como vantagem do painel, pode-se citar o seu maior potencial analítico em relação à amostra independente, porque é possível coletar um número maior de informações, divididas pelas várias ocasiões. Pode-se também detectar erros nas respostas com maior 
facilidade, uma vez que um informante pode fornecer a mesma informação mais de uma vez.

Além disso, a experiência tem mostrado que os informantes têm mais boa vontade e fornecem dados mais aprimorados na segunda entrevista, principalmente quando as informaçôes são técnicas ou confidenciais. Outra vantagem do painel é o custo mais baixo na obtenção de futuras observações.

Por outro lado, os principais problemas apresentados pelo painel é que os informantes podem ficar cansados de fornecer sempre o mesmo tipo de informação e, desse modo, aumentar o número de não-respostas, ou, com o passar do tempo, tornarem-se influenciados pelo pesquisador.

Ainda como desvantagem, o painel não leva em conta que a composição da população pode estar mudando e desconsidera a introdução de novos elementos na população.

Pode ainda ocorrer a dificuldade de se entrar em contato com os informantes periodicamente, uma vez que pode haver mudança de endereço ou mortalidade do informante. Além disso, pode ocorrer dificuldade em seguir com o painel quando há divisão da unidade observacional, como por exemplo família.

Em todos esses casos, o que é vantagem para o painel é desvantagem para a amostra independente e vice-versa.

Pode-se ainda acrescentar que a amostra independente torna os cálculos mais simples do que no caso do painel e possibilita o acúmulo de informações para o estudo de populações raras.

Algumas vezes o painel rotativo apresenta melhores resultados do que o painel ou a amostra independente. Na verdade, a escolha do plano amostral depende em parte do objetivo do problema em estudo. Duncan e Kalton (1987) listaram alguns objetivos e 
apresentaram, para cada um, qual o plano amostral mais adequado.

Neste trabalho nos restringiremos à utilização de imputação em painéis. O caso univariado corresponde àquele em que somente uma característica é medida. O painel multivariado é o painel onde várias características são medidas para cada indivíduo selecionado, em cada um dos instantes pesquisados.

A maioria dos trabalhos com medidas repetidas, como por exemplo o trabalho de Laird e Ware (1982), usam modelos lineares. Segundo Little (1988), dois modelos dominam a literatura: o primeiro, denominado por ele "multivariate repeated measures model" é dado por $\mathrm{Y}_{i}=\mathrm{X}_{i} \beta+\epsilon_{i}$, com $\beta$ fixo e $\epsilon_{i}$ independentes, com distribuição normal multivariada com média zero e matriz de covariâncias $\Sigma$ e supõe que valores de $\mathbf{Y}$ podem ser perdidos. Esse modelo foi discutido por Laird (1988) e Schluchter (1988) e permite que $\Sigma$ seja indexada por um $i$. O segundo, denominado por ele "random slopes model" assume que os $\beta_{i}$ 's são aleatórios, independentes e tem distribuição normal multivariada. Foi utilizado, por exemplo, por Wu e Bailey (1988).

O modelo misto, de efeitos fixos e aleatórios (ver Winer (1971)), tem sido intensamente utilizado. Entre os autores que trabalharam com esse modelo e dados completos podemos citar Henderson (1975), Wang (1983) e Jeske e Harville (1988). Outros, como Chi e Reinsel (1989) e Harville e Carriquiry (1992) consideraram dados não balanceados, que pode acomodar o caso de não-resposta. Esse modelo ainda foi usado por Box e Tiao (1968) sob o enfoque Bayesiano e por Lindstrom e Bates (1988), que implementaram os algoritmos de Newton-Raphson e EM.

Entre os autores que consideraram o painel univariado e modelos de curva de crescimento estão Khatri (1966), Rao $(1965,1967,1987)$, Grizzle e Allen (1969) e Kleimbaum (1973) que generalizou o modelo proposto por Potthoff e Roy (1964) para admitir da- 
dos incompletos. Seu método consiste em reduzir o modelo de curva de crescimento ao modelo de mínimos quadrados ponderados univariado com matriz de covariâncias $\Sigma$ desconhecida e a usual suposição de normalidade.

Reinsel $(1982,1984,1985)$ fez uma extensão do modelo de Potthoff e Roy (1964) para o caso multivariado em que $m$ características são medidas em $p$ ocasiões diferentes. Ele apresentou resultados de estimação e teste de hipóteses para os parâmetros fixos do modelo, estudou a previsão de efeitos aleatórios individuais e também a previsão de observações futuras dadas as observações passadas. Considerou normalidade dos efeitos aleatórios e independência entre os indivíduos.

Alguns trabalhos consideraram a função de verossimilhança sob a suposição de normalidade. Liski (1985) apresentou um método computacional para analisar modelos de curva de crescimento usando o algoritmo EM e análise de covariância. Srivastava (1985) obteve as equações de verossimilhança para vários modelos, estabelecendo a consistência dos estimadores e deduzindo explicitamente o teste da razão de verossimilhança. Jennrich e Schluchter (1986) descreveram os métodos de Newton-Raphson e Scoring de Fisher para computar o estimador de máxima verossimilhança e um algoritmo EM generalizado para computar o estimador de máxima verossimilhança restrito. Laird (1988) fez uma revisão sobre o uso de análises baseadas na função de verossimilhança para dados longitudinais com observações perdidas. Wansbeek e Kapteyn (1989) discutiram a estimação de efeitos fixos e apresentaram um estimador quadrático não-viesado e o estimador de máxima verossimilhança para o modelo de componentes de erro. Park e Woolson (1992) sugeriram dois métodos de estimação dos parâmetros do modelo com fator fixo, um de mínimos quadrados generalizados baseado num método não iterativo e o outro baseado no algoritmo EM. 
Outros modelos também foram estudados. Ware (1985) fez uma discussão sobre três famílias de modelos para dados longitudinais: modelos multivariados, autoregressivos e de efeitos aleatórios. Nesse trabalho, o autor supôs normalidade e dados completos, permitindo que os individuos pudessem ser observados em um número de instantes diferentes. Já Gilks, Wang, Yvonnet e Coursaget (1993) propuseram uma generalização do modelo de Laird e Ware (1982) para acomodar efeitos aleatórios múltiplos e mostraram como a amostragem de Gibbs pode ser usada para estimá-los. Alguns autores como Stiratelli, Laird e Ware (1984), Liang e Zeger (1986) e Zeger, Liang e Albert (1988) estenderam os resultados para modelos lineares generalizados, enquanto que Lindstrom e Bates (1990) usaram modelos não lineares e definiram estimadores dos parâmetros que são combinações lineares de estimadores de mínimos quadrados para modelos não lineares com estimadores de máxima verossimilhança (ou estimadores de máxima verossimilhança restritos) de modelos lineares, usando o método de Newton-Raphson.

Sob o enfoque clássico, no estudo de populações finitas, poucos trabalhos são encontrados na literatura. Bouza (1983) obteve um estimador não-viesado para a diferença das médias populacionais de duas variáveis com observações perdidas, usando amostra aleatória simples com reposição em um instante, Singh (1985) sugeriu um procedimento de estimação do total da população baseado em dados longitudinais incompletos e Chiu e Sedransk (1986) propuseram um método de imputação assumindo uma amostra de $n$ indivíduos sorteados com reposição de uma população de tamanho $N$, que pode assumir os valores $Y_{1}, Y_{2}, \ldots, Y_{D} . \mathrm{O}$ procedimento sugerido consiste em estimar o número de não-respondentes cuja resposta é $Y_{i}$, para $i=1,2, \ldots, D$. A restrição a esse método é que ele supõe que os valores que $Y$ pode assumir, ou seja, $Y_{1}, Y_{2}, \ldots, Y_{D}$, podem ser especificados antes da amostragem. 
Nosso objetivo neste trabalho é estudar a imputação de dados em painéis sorteados de populaçôes finitas, tratanto os casos univariado e multivariado. Especificamente, tentamos resolver o seguinte problema:

$i$. uma amostra de $n$ indivíduos é sorteada, sem reposição, de uma população finita de tamanho $N$;

ii . cada indivíduo é observado em $T$ instantes de tempo;

iii . a observação correspondente ao último indivíduo no último instante de tempo é perdida. Queremos prever essa informação.

O conjunto de dados pode ser representado pelo seguinte esquema:

\begin{tabular}{c|cccc|c} 
& \multicolumn{5}{|c|}{ tempo } \\
indiv. & 1 & 2 & $\cdots$ & $T-1$ & $T$ \\
\hline 1 & $y_{11}$ & $y_{21}$ & $\cdots$ & $y_{(T-1), 1}$ & $y_{T 1}$ \\
2 & $y_{12}$ & $y_{22}$ & $\cdots$ & $y_{(T-1), 2}$ & $y_{T 2}$ \\
$\vdots$ & $\vdots$ & $\vdots$ & $\cdots$ & $\vdots$ & $\vdots$ \\
$n-1$ & $y_{1,(n-1)}$ & $y_{2,(n-1)}$ & $\cdots$ & $y_{(T-1),(n-1)}$ & $y_{T,(n-1)}$ \\
\hline$n$ & $y_{1 n}$ & $y_{2 n}$ & $\cdots$ & $y_{(T-1), n}$ & $? ?$
\end{tabular}

Uma das maneiras de prever $y_{T_{n}}$ é imputar-lhe a média das $n-1$ observações no $T$-ésimo instante. Outra, a média das $T-1$ observações do $n$-ésimo indivíduo.

Exemplo 1.1. A tabela 3.1 a seguir mostra os dados de total de salários (em milhões de cruzeiros reais) de cinco indústrias do setor de Papel e Papelão do Estado de São Paulo, do período de janeiro a abril de 1994. Quatro das empresas têm os dados completos, enquanto que a empresa $n^{\circ} 5$ tem o dado de abril perdido. 
TABELA 1.1: DADOS

IND.PAPEL E PAPELÃO DO ESTADO DE SÃO PAULO

(Estrato: empresa com mais de 500 empregados)

TOTAL DE SALÁRIOS NOMINAIS (em milhões)

\begin{tabular}{|l|r|r|r|r|}
\hline & \multicolumn{4}{|c|}{ MESES/94 } \\
\cline { 2 - 5 } & JAN & FEV & MAR & ABR \\
\hline EMPRESA 1 & 1.072 & 1.661 & 1.797 & 1.837 \\
EMPRESA 2 & 667 & 1.250 & 1.482 & 1.987 \\
EMPRESA 3 & 687 & 1.113 & 1.919 & 2.283 \\
EMPRESA 4 & 442 & 765 & 1.238 & 1.432 \\
EMPRESA 5 & 157 & 242 & 426 & \\
\hline
\end{tabular}

Fonte: FIESP

A média no $4^{4}$ instante é 1885 enquanto que a média da $5 \underline{a}$ empresa, 275.

Nosso objetivo é obter o "melhor" previsor das observações ausentes. O conceito de "melhor" será definido mais adiante.

Adotamos o modelo misto de efeitos fixos e aleatórios e a nossa principal contribuição é apresentar o BLUP (Best Linear Unbiased Predictor) para os efeitos fixos, aleatórios e para as observações perdidas, relaxando as suposições usuais de normalidade e independência dos efeitos.

No modelo que adotamos, consideramos o tempo como um fator fixo e os indivíduos como um fator aleatório sorteado de uma população finita.

No capítulo 2 trabalhamos com um modelo misto mais geral e obtemos o BLUP para o caso de variâncias conhecidas. No capítulo 3, estudamos o caso univariado, aplicando os resultados obtidos no capítulo 2 ao nosso modelo, primeiro para o caso de uma observação perdida, depois para o caso de várias não-respostas em um só instante de tempo e por último, para o caso de várias observações perdidas em qualquer instante de tempo. Apresentamos o erro quadrático médio dos previsores e fazemos uma conexão com o caso 
de populações infinitas.

No capítulo 4 , fazemos a extensão para o caso multivariado. No capítulo 5 , fazemos a extensão para o caso de variâncias desconhecidas, considerando os seguintes métodos de estimação das componentes de variância: ANOVA (Analysis of Variance), REML (Restricted Maximum Likelihood) e MINQUE (Minimum Norm Quadratic Unbiased Estimation).

Para finalizar ilustramos com uma aplicação numérica, apresentando nossas conclusões e possiveis tópicos para pesquisas futuras. 


\section{CAPÍTULO 2}

\section{BLUP (BEST LINEAR UNBIASED PREDICTOR) PARA O MODELO MISTO}

\subsection{INTRODUÇÃO}

O objetivo neste capítulo é obter o BLUP (Best Linear Unbiased Predictor) para as observações perdidas, os efeitos fixos e os efeitos aleatórios, para o caso de variâncias conhecidas e a adoção do modelo linear misto.

Segundo Wang (1983) as principais abordagens para a análise de dados de medidas repetidas são o modelo misto e o modelo multivariado. Estatisticamente, a diferença entre eles reside nas suposições feitas, com o modelo multivariado não assumindo conhecimento a priori da estrutura de covariância das medidas repetidas. Segundo ele, o modelo multivariado é menos poderoso, principalmente quando o número de observações é pequeno. 
A estimação e previsão de efeitos fixos e aleatórios tem sido tratadas por vários autores. Scheffé (1956) e Searle (1988) contam um pouco da história dos modelos de efeitos aleatórios, também conhecidos como modelos de componentes de variância. Os primeiros trabalhos sobre estimação de efeitos fixos foram os de Legendre (1806) e de Gauss (1809) e apareceram em livros de Astronomia. Os modelos de componentes de variância também tiveram sua primeira aparição na área de Astronomia com os trabalhos de Airy (1861) e Chauvenet (1863). Em ciências biológicas, o primeiro a usá-lo foi Fisher (1918), na área de genética. A especificação clara de modelos mistos começou com Eisenhart (1947). Atualmente existe uma vasta literatura sobre o assunto. Khuri e Sahai (1985) apresentam uma bibliografia a respeito e umas das principais referências são os livros de Searle (1971, 1987), Searle, Casella e McCulloch (1992) e de Winer (1971).

Outros que justificam o uso do modelo misto são Harville e Carriquiry (1992). Segundo eles "If (as in a typical animal breeding application) the variance ratio is small and the data are limited in extent or are highly unbalanced, the results produced by BLUP can be markedly superior to those obtained by treating the random effects as fixed and employing ordinary least squares methods".

A principal diferença entre o modelo com fator aleatório e o modelo com fator fixo é que, no último, as conclusões referem-se somente aos níveis incluídos no experimento, enquanto que no modelo aleatório, as conclusões devem ser estendidas a toda a população de níveis da qual a amostra foi retirada.

Para medidas repetidas, os modelos mistos tornaram-se populares porque permitem o uso de uma estrutura de covariância repetida flexível. A noção de que respostas individuais seguem uma função similar com parâmetros variando individualmente parece apropriada para muitas situações. De qualquer forma, o sucesso de qualquer método depende da 
validade do modelo assumido.

Ainda como referência sobre a utilização de modelos mistos, podemos citar Henderson (1950, 1963, 1965, 1975), Goldberger (1962), Box e Tiao (1968), Lindley e Smith (1972), Harville (1976, 1977, 1990), Demplle (1977), Fellner (1986, 1987), Jeske e Harville (1988), Lindstrom e Bates (1988), Chi e Reinsel (1989), Schall (1991), Robinson (1991) e Harville e Carriquiry (1992).

Em geral, o modelo adotado tem sido o seguinte:

$$
\mathrm{y}=\mathrm{X} \alpha+\mathrm{Zb}+\mathrm{e}
$$

onde

y: vetor de $d$ variáveis aleatórias observáveis;

$\alpha$ : vetor de $p$ parâmetros fixos desconhecidos (efeitos fixos);

b: vetor de $q$ variáveis aleatórias não observáveis (efeitos aleatórios);

$\mathrm{X}$ e Z: matrizes conhecidas;

e: vetor de variáveis aleatórias (erros),

com as seguintes suposições:

$$
\begin{array}{ll}
\mathbb{E}(\mathrm{b})=0 & \mathbb{E}(\mathrm{e})=0 \\
\operatorname{Var}(\mathrm{b})=\mathrm{G} \sigma^{2} & \operatorname{Var}(\mathrm{e})=\mathrm{R} \sigma^{2} \\
\operatorname{Cov}(\mathrm{b}, \mathrm{e})=0 &
\end{array}
$$

onde $\mathbf{G}$ e $\mathbf{R}$ são matrizes positivas definidas de dimensão $(q \times q)$ e $(d \times d)$, respectivamente. Além disso, estamos supondo que $\mathbf{G}$ e $\mathbf{R}$ são matrizes de posto completo.

Esse modelo engloba uma série de modelos encontrados na literatura. Como ilustração, damos os seguintes exemplos: 
Exemplo 2.1. Modelo simples de efeitos fixos e aleatórios

$$
y_{t i}=\mu+\alpha_{t}+b_{i}+e_{t i}
$$

onde

$y_{t i}$ : observação do $i$-ésimo indivíduo no t-ésimo instante;

$\mu$ : média geral;

$\alpha_{t}$ : fator fixo medindo o efeito do tempo;

$b_{i}$ : fator aleatório medindo o efeito do indivíduo;

$e_{t i}$ : erro associado ao i-ésimo indivíduo no t-ésimo instante.

Este modelo será tratado com detalhes nos capítulos 3 e 4 .

Exemplo 2.2. Modelos de curvas de crescimento

Suponha que dois indivíduos são observados em três instantes de tempo $\left(t_{1}, t_{2}\right.$ e $\left.t_{3}\right)$, e estamos ajustando uma reta no tempo para cada indivíduo. O modelo será dado por:

$$
y_{t i}=(a+b t)+\left(a_{i}+b_{i} t\right)+e_{t i}
$$

ou seja,

$$
\mathrm{y}=\mathrm{X} \alpha+\mathrm{Zb}+\mathrm{e}
$$

com 


$$
\mathbf{y}=\left(\begin{array}{l}
y_{11} \\
y_{21} \\
y_{31} \\
y_{12} \\
y_{22} \\
y_{32}
\end{array}\right) \quad \mathbf{X}=\left(\begin{array}{ll}
1 & t_{1} \\
1 & t_{2} \\
1 & t_{3} \\
1 & t_{1} \\
1 & t_{2} \\
1 & t_{3}
\end{array}\right) \quad \alpha=\left(\begin{array}{l}
a \\
b
\end{array}\right)
$$

e

$$
\mathrm{Z}=\left(\begin{array}{cccc}
1 & t_{1} & 0 & 0 \\
1 & t_{2} & 0 & 0 \\
1 & t_{3} & 0 & 0 \\
0 & 0 & 1 & t_{1} \\
0 & 0 & 1 & t_{2} \\
0 & 0 & 1 & t_{3}
\end{array}\right) \quad \mathbf{b}=\left(\begin{array}{c}
a_{1} \\
b_{1} \\
a_{2} \\
b_{2}
\end{array}\right) \quad \mathrm{e}=\left(\begin{array}{l}
e_{11} \\
e_{21} \\
e_{31} \\
e_{12} \\
e_{22} \\
e_{32}
\end{array}\right)
$$

Com a suposição de erros independentes e mesma matriz de covariâncias para os efeitos individuais, $\mathbf{R}$ e $\mathbf{G}$ são:

$$
\mathbf{R}=\operatorname{Var}(\mathbf{e})=\mathbf{I} \quad \text { e } \quad \mathbf{G}=\operatorname{Var}(\mathbf{b})=\left(\begin{array}{cccc}
S_{a}^{2} & S_{a b} & S_{a a} & S_{a b} \\
S_{a b} & S_{b}^{2} & S_{a b} & S_{b b} \\
S_{a a} & S_{a b} & S_{a}^{2} & S_{a b} \\
S_{a b} & S_{b b} & S_{a b} & S_{b}^{2}
\end{array}\right)
$$

onde

$$
\begin{array}{ll}
S_{a}^{2}=\operatorname{Var}\left(a_{i}\right), & i=1,2 \\
S_{b}^{2}=\operatorname{Var}\left(b_{i}\right), & i=1,2 \\
S_{a a}=\operatorname{Cov}\left(a_{i}, a_{j}\right), & i, j=1,2, i \neq j \\
S_{b b}=\operatorname{Cov}\left(b_{i}, b_{j}\right), & i, j=1,2, i \neq j \\
S_{a b}=\operatorname{Cov}\left(a_{i}, b_{j}\right), & i, j=1,2 .
\end{array}
$$

Alguns pesquisadores fazem suposições sobre a distribuição dos erros e dos efeitos aleatórios utilizando, em geral, a distribuição normal. Outros, usam métodos que requerem somente suposições sobre os dois primeiros momentos. 
Dessas suposições tem-se que:

$$
\operatorname{Var}(\mathrm{y})=(\mathbf{R}+\mathbf{Z G Z}) \sigma^{2}=\mathrm{W} \sigma^{2}
$$

onde

$$
\mathrm{W}=\mathrm{R}+\mathrm{ZGZ} \text {. }
$$

Geralmente é assumido que, com exceção do parâmetro $\sigma^{2}$, a estrutura de covariâncias, isto é, $\mathrm{G}$ e $\mathrm{R}$, é conhecida.

Alguns autores fazem distinção entre os termos estimador e previsor, sendo estimador utilizado para efeitos fixos e previsor para efeitos aleatórios. Dentro do possível, faremos esta distinção mas quando nos referirmos a ambos os efeitos ou a uma combinação linear deles, usaremos a palavra previsor.

As definições a seguir introduzem o conceito de previsor não-viesado, erro quadrático médio e o critério de comparação entre dois previsores.

Definição 2.1 - Previsor não-viesado

$O$ previsor $\hat{\theta}$ de $\theta$ é dito ser não-viesado para $\theta$ se

$$
\mathbb{I E}(\hat{\theta}-\theta)=0
$$

\section{Definição 2.2 - Erro Quadrático Médio (EQM)}

$O$ erro quadrático médio de $\hat{\theta}$, previsor de $\theta$,é definido como

$$
E Q M(\hat{\theta})=\mathbb{E}\left[(\hat{\theta}-\theta)(\hat{\theta}-\theta)^{\prime}\right]
$$




\section{Definição 2.3}

Sejam $\mathbf{A}$ e $\mathbf{B}$ duas matrizes de mesma dimensão. Dizemos que $\mathbf{A}$ é maior do que $\mathbf{B}$ se $\mathbf{A}-\mathbf{B}$ for uma matriz definida não-negativa.

A definição 2.1 é similar à definição de previsor não-viesado utilizada sob o enfoque da Teoria de Superpopulações uma vez que prever b significa prever uma realização da variável $b$. A diferença é que, neste caso, a esperança está sendo calculada sob a abordagem clássica, segundo a distribuição gerada pelo planejamento amostral. Para o estimador $\hat{\alpha}$ de $\alpha$, dizer que $\hat{\alpha}$ é não-viesado para $\alpha$ equivale a dizer que $\mathbb{E}(\hat{\alpha})=\alpha$, pois $\alpha$ é fixo.

O erro quadrático médio é uma medida tradicionalmente usada na teoria de previsão para avaliar o comportamento de previsores ou fazer comparações entre eles.

Os principais critérios utilizados para comparar estimadores de vetores são: M-otimalidade, T-otimalidade e D-otimalidade. Kale e Chandrasekar (1983) fazem uma discussão sobre a equivalência desses critérios e Subramani (1991) as define.

O critério que escolhemos para cada um dos previsores foi o de M-otimalidade e corresponde ao seguinte.

M-otimalidade: se $\hat{\theta}_{1}$ e $\hat{\theta}_{2}$ são dois previsores não-viesados de $\theta$ e $\mathbf{A}_{1}$ e $\mathbf{A}_{2}$ as respectivas matrizes de erro quadrático médio, dizemos que $\hat{\theta}_{1}$ é "melhor" do que $\hat{\theta}_{2}$ se $\mathbf{A}_{2}$ é maior do que $\mathrm{A}_{1}$ no sentido da definição 2.3.

Isto significa que o erro quadrático médio de qualquer combinação linear dos componentes de $\hat{\theta}_{1}$ é menor ou igual ao erro quadrático médio dessa mesma combinação linear aplicada às componentes de $\hat{\theta}_{2}$. Esse critério é equivalente ao utilizado por Bolfarine e 
Zacks (1992) e Elian (1992), com base no conceito de erro quadrático médio generalizado proposto por Tam (1986).

Os critérios de T-otimalidade e D-otimalidade são baseados, respectivamente, no traço e no determinante das matrizes de erro quadrático médio. O "melhor" previsor é aquele que tem o menor traço (T-otimalidade) ou o menor determinante (D-otimalidade).

O BLUP (Best Linear Unbiased Predictor) utilizado neste contexto, é linear porque é uma função linear dos dados observados, é não-viesado no sentido da definição 2.1 , isto é, a esperança do previsor é igual à esperança da quantidade prevista e é o melhor no sentido de que é aquele que tem o menor erro quadrático médio (M-otimalidade) na classe dos previsores lineares não-viesados.

O BLUP pode ser deduzido de várias maneiras diferentes. Matematicamente, os BLUP's de $\alpha$ e b são as soluções das seguintes equações, chamadas de equações do modelo misto, dadas por Henderson (1950),

$$
\begin{aligned}
& X^{\prime} R^{-1} X \hat{\alpha}+X^{\prime} R^{-1} Z \hat{b}=X^{\prime} R^{-1} y \\
& Z^{\prime} R^{-1} X \hat{\alpha}+\left(Z^{\prime} R^{-1} Z+G^{-1}\right) \hat{b}=Z^{\prime} R^{-1} y
\end{aligned}
$$

As soluções, conhecidas como soluções do modelo misto, são:

$$
\begin{gathered}
\hat{\alpha}=\left(\mathrm{X}^{\prime} \mathrm{W}^{-1} \mathrm{X}\right)^{-1} \mathrm{X}^{\prime} \mathrm{W}^{-1} \mathrm{y} \\
\hat{\mathrm{b}}=\left(\mathrm{Z}^{\prime} \mathrm{R}^{-1} \mathrm{Z}+\mathrm{G}^{-1}\right)^{-1}\left[\mathrm{Z}^{\prime} \mathrm{R}^{-1}-\mathrm{Z}^{\prime} \mathrm{R}^{-1} \mathrm{X}\left(\mathrm{X}^{\prime} \mathrm{W}^{-1} \mathrm{X}\right)^{-1} \mathrm{X}^{\prime} \mathrm{W}^{-1}\right] \mathrm{y} .
\end{gathered}
$$

Harville (1977) mostrou que o BLUP de uma combinação linear de efeitos fixos e aleatórios é a mesma combinação linear aplicada aos previsores dos efeitos, dados por 
(2.5) e (2.6)

Henderson (1950) obteve o BLUP através da maximização da função densidade conjunta de $\mathrm{b}$ e y com respeito a $\alpha$ e b e sugeriu o nome de estimador de máxima verossimilhança conjunta, uma vez que a função que ele maximizou não é a função de verossimilhança no sentido convencional porque se baseia na variável b não-observável. A função a ser maximizada é

$$
\begin{array}{r}
\left(2 \pi \sigma^{2}\right)^{-\frac{1}{2} d-\frac{1}{2} q}\left(\operatorname{det}\left[\begin{array}{cc}
\mathrm{G} & 0 \\
0 & \mathrm{R}
\end{array}\right]\right)^{-\frac{1}{2}} \exp \left\{-\frac{1}{2 \sigma^{2}}\left(\begin{array}{c}
\mathrm{b} \\
\mathrm{y}-\mathrm{X} \alpha-\mathrm{Zb}
\end{array}\right)^{\prime}\left(\begin{array}{cc}
\mathrm{G} & 0 \\
0 & \mathrm{R}
\end{array}\right)^{-1}\right. \\
\left.\cdot\left(\begin{array}{c}
\mathrm{b} \\
\mathrm{y}-\mathrm{X} \alpha-\mathrm{Zb}
\end{array}\right)\right\},
\end{array}
$$

que equivale a mininizar

$$
\left(\begin{array}{c}
\mathrm{b} \\
\mathrm{y}-\mathrm{X} \alpha-\mathrm{Zb}
\end{array}\right)^{\prime}\left(\begin{array}{cc}
\mathrm{G} & 0 \\
0 & \mathrm{R}
\end{array}\right)^{-1}\left(\begin{array}{c}
\mathrm{b} \\
\mathrm{y}-\mathrm{X} \alpha-\mathrm{Zb}
\end{array}\right)
$$

Sob o ponto de vista Bayesiano, os mesmos resultados são obtidos quando consideramos para $\alpha$ uma densidade a priori uniforme e para $\mathrm{b}$ uma densidade a priori com média zero e variância $\mathbf{G} \sigma^{2}$, independente de $\alpha$. Sob esse enfoque, Box e Tiao (1968) estudaram o problema de estimação da média considerando independência e normalidade para erros e efeitos.

Goldberger (1962) e Henderson (1963) utilizaram multiplicadores de Lagrange para obter o BLUP de combinações lineares dos efeitos. Potthoff e Roy (1964), Rao (1965, 1967) e Grizzle e Allen (1969) consideraram dados balanceados. Laird e Ware (1982) concentraram-se no caso de erros independentes enquanto que Henderson (1975) relaxou 
as condições de normalidade e independência dos efeitos, trabalhando entretanto com dados completos. Chi e Reinsel (1989) consideraram erros autocorrelacionados sob uma estrutura auto-regressiva $(\mathrm{AR}(1))$ e desenvolveram um teste score para autocorrelação no modelo de efeitos aleatórios condicionalmente independentes, dando um procedimento de estimação de máxima verossimilhança. Também consideraram estimação de Bayes empírica e previsão de observações futuras.

Harville (1976) considerou o estimador de combinações lineares de $\alpha$ e $\beta, \lambda_{1} \alpha+\lambda_{2} \beta$, onde $\beta$ é uma realização de b e $\lambda_{1} \alpha$ é estimável e estendeu o teorema de Gauss-Markov para incluir a previsão de efeitos aleatórios, considerando o caso de posto incompleto. Posteriormente, Harville e Carriquiry (1992) consideraram procedimentos de previsão por ponto e intervalo para combinações lineares de $\alpha$ e $\beta$ em um modelo misto com duas componentes de variância. Nesse trabalho eles descreveram a abordagem clássica e a Bayesiana, usando na clássica o estimador de máxima verossimilhança restrito para a razão entre as variâncias e supuseram normalidade para efeitos e erros.

Fellner $(1986,1987)$ propôs um algoritmo para encontrar os estimadores de máxima verossimilhança dos efeitos e das componentes de variância para o modelo normal. Schall (1991) estendeu esse algoritmo para modelos lineares generalizados.

Ainda sob o ponto de vista computacional, Lindstrom e Bates (1988) desenvolveram uma implementação do algoritmo de Newton-Raphson para estimar os parâmetros do modelo misto para medidas repetidas. Eles formularam as derivadas para a estimação de máxima verossimilhança e para a estimação de máxima verossimilhança restrita, implementaram os algoritmos de Newton-Raphson e EM, compararam os dois métodos em dois conjuntos de dados e concluíram que, em muitas situações, um bem-implementado Newton-Raphson é melhor do que o EM. Tudo isso foi desenvolvido sob a suposição de 
normalidade.

Jeske e Harville (1988) apresentaram uma abordagem mais geral para obter intervalos de previsão aproximados para uma variável aleatória não-observável $W$ baseada em uma variável observável $Y$. Para isso, assumiram que a esperança de $W$ e $Y$ são combinações lineares de parâmetros desconhecidos e que a distribuição conjunta é simétrica. Esse seria o caso do modelo linear misto com suposição de normalidade para efeitos e erros e $W$ seria uma combinação linear de efeitos fixos e aleatórios.

O enfoque mais intuitivo foi dado por Harville (1990). Ele considerou a classe de previsores lineares e mostrou, através do cálculo direto das esperanças envolvidas, que os previsores dados por (2.5) e (2.6) são BLUP.

Robinson (1991) fez uma discussão muito interessante sobre o assunto. Ele descreveu quatro maneiras diferentes de obter o BLUP, duas delas assumindo suposição de normalidade e duas não. Além disso, ele fez uma conexão com outras teorias e mostrou algumas aplicações de previsão de efeitos aleatórios.

Em todos esses trabalhos, o BLUP foi obtido para dados completos. Para dados com observações incompletas, podemos citar o trabalho de Singh e Pratap (1989), onde eles obtém os estimadores de mínimos quadrados para os efeitos fixos e a observação perdida.

\subsection{ESTIMAÇÃO E PREVISÃO COM VARIÂNCIAS CONHECIDAS}

Nesta seção deduzimos o BLUP para efeitos fixos e aleatórios e para as observações perdidas, quando a razão entre as componentes da variância é conhecida. Estudamos o caso em que $m$ observações são perdidas e os erros correspondentes às observações 
presentes e ausentes são não correlacionados. $\mathrm{Na}$ dedução, combinamos as idéias de Henderson (1950), Singh e Pratap (1989) e Harville (1990).

O modelo adotado é o modelo misto dado por (2.1). Estamos supondo que $m$ das $d$ observações são ausentes e, sem perda de generalidades, supomos que estas sejam as últimas observações do vetor y. Assim, podemos partir o modelo nas partes observada e ausente, de modo que:

$$
\mathrm{y}=\left(\begin{array}{c}
\mathrm{y}_{o} \\
\mathrm{y}_{a}
\end{array}\right)=\left(\begin{array}{c}
\mathrm{X}_{o} \\
\mathrm{X}_{a}
\end{array}\right) \alpha+\left(\begin{array}{c}
\mathrm{Z}_{o} \\
\mathrm{Z}_{a}
\end{array}\right) \mathrm{b}+\left(\begin{array}{c}
\mathrm{e}_{o} \\
\mathrm{e}_{a}
\end{array}\right) .
$$

Seguindo as idéias de Henderson (1950), queremos obter $\alpha$, b e y que minimize a seguinte expressão:

$$
\left(\begin{array}{c}
\mathrm{b} \\
\mathrm{y}_{o}-\mathrm{X}_{o} \alpha-\mathrm{Z}_{o} \mathrm{~b} \\
\mathrm{y}_{a}-\mathrm{X}_{a} \alpha-\mathrm{Z}_{a} \mathrm{~b}
\end{array}\right)^{\prime}\left(\begin{array}{ccc}
\mathrm{G}^{-1} & 0 & 0 \\
0 & \mathrm{R}_{o}^{-1} & 0 \\
0 & 0 & \mathrm{R}_{a}^{-1}
\end{array}\right)\left(\begin{array}{c}
\mathrm{b} \\
\mathrm{y}_{o}-\mathrm{X}_{o} \alpha-\mathrm{Z}_{o} \mathrm{~b} \\
\mathrm{y}_{a}-\mathrm{X}_{a} \alpha-\mathrm{Z}_{a} \mathrm{~b}
\end{array}\right)
$$

A idéia de minimizar essa expressão é a mesma que aparece na obtenção do estimador de mínimos quadrados generalizados e também a mesma para obter o estimador de máxima verossimilhança quando b e e são independentes e têm distribuição normal multivariada com média zero e matriz de covariâncias $\mathrm{G} \sigma^{2}$ e $\mathrm{R} \sigma^{2}$ para b e e, respectivamente.

Posteriormente mostraremos que $\hat{\alpha}, \hat{\mathrm{b}}$ e $\hat{\mathrm{y}}_{a}$ que minimizam a expressão (2.9) são os BLUP's para os correspondentes parâmetros, mesmo que não tenhamos a suposição de normalidade.

Minimizar (2.9) é equivalente a minimizar

$$
\begin{aligned}
f=\mathrm{b}^{\prime} \mathrm{G}^{-1} \mathrm{~b} & +\left(\mathrm{y}_{\circ}-\mathrm{X}_{\circ} \alpha-\mathrm{Z}_{\circ} \mathrm{b}\right)^{\prime} \mathbf{R}_{o}^{-1}\left(\mathrm{y}_{\circ}-\mathrm{X}_{o} \alpha-\mathrm{Z}_{o} \mathrm{~b}\right) \\
& +\left(\mathrm{y}_{a}-\mathrm{X}_{a} \alpha-\mathrm{Z}_{a} \mathrm{~b}\right)^{\prime} \mathbf{R}_{a}^{-1}\left(\mathrm{y}_{a}-\mathrm{X}_{a} \alpha-\mathrm{Z}_{a} \mathrm{~b}\right) .
\end{aligned}
$$


Derivando (2.10) com relação a $\alpha$, b e $\mathrm{y}_{a}$ (ver apêndice (A.5) e Magnus e Neudecker (1988)), temos:

$$
\begin{gathered}
\frac{\partial f}{\partial \alpha}=-2 \mathrm{X}_{o}^{\prime} \mathbf{R}_{o}^{-1}\left(\mathrm{y}_{o}-\mathrm{X}_{\circ} \hat{\alpha}-\mathrm{Z}_{o} \hat{\mathrm{b}}\right)-2 \mathrm{X}_{a}^{\prime} \mathbf{R}_{a}^{-1}\left(\hat{\mathrm{Y}}_{a}-\mathrm{X}_{a} \hat{\alpha}-\mathrm{Z}_{a} \hat{\mathrm{b}}\right)=0 \\
\frac{\partial f}{\partial \mathrm{b}}=2 \mathrm{G}^{-1} \hat{\mathrm{b}}-2 \mathrm{Z}_{o}^{\prime} \mathrm{R}_{o}^{-1}\left(\mathrm{y}_{o}-\mathrm{X}_{o} \hat{\alpha}-\mathrm{Z}_{o} \hat{\mathrm{b}}\right)-2 \mathrm{Z}_{a}^{\prime} \mathbf{R}_{a}^{-1}\left(\hat{\mathrm{y}}_{a}-\mathrm{X}_{a} \hat{\alpha}-\mathrm{Z}_{a} \hat{\mathrm{b}}\right)=0 \\
\frac{\partial f}{\partial \mathrm{y}_{a}}=2 \mathrm{R}_{a}^{-1}\left(\hat{\mathrm{y}}_{a}-\mathrm{X}_{a} \hat{\alpha}-\mathrm{Z}_{a} \hat{\mathrm{b}}\right)=0 .
\end{gathered}
$$

De (2.12) temos que:

$$
\mathrm{G}^{-1} \hat{\mathrm{b}}+\mathrm{Z}_{o}^{\prime} \mathbf{R}_{o}^{-1} \mathrm{Z}_{o} \hat{\mathrm{b}}+\mathrm{Z}_{a}^{\prime} \mathrm{R}_{a}^{-1} \mathrm{Z}_{a} \hat{\mathrm{b}}=\left(-\mathrm{Z}_{o}^{\prime} \mathrm{R}_{o}^{-1} \mathrm{X}_{o}-\mathrm{Z}_{a}^{\prime} \mathbf{R}_{a}^{-1} \mathrm{X}_{a}\right) \hat{\alpha}+\mathrm{Z}_{o}^{\prime} \mathbf{R}_{o}^{-1} \mathrm{y}_{o}+\mathrm{Z}_{a}^{\prime} \mathbf{R}_{a}^{-1} \hat{\mathbf{y}}_{a}
$$

ou seja,

$$
\hat{\mathrm{b}}=\left(\mathrm{Z}^{\prime} \mathrm{R}^{-1} \mathrm{Z}+\mathrm{G}^{-1}\right)^{-1}\left(\mathrm{Z}_{o}^{\prime} \mathrm{R}_{o}^{-1} \mathrm{y}_{o}+\mathrm{Z}_{a}^{\prime} \mathrm{R}_{a}^{-1} \hat{\mathrm{Y}}_{a}-\mathrm{Z}^{\prime} \mathrm{R}^{-1} \mathrm{X} \hat{\alpha}\right)
$$

Substituindo (2.14) em (2.11) temos

$$
\begin{aligned}
& \mathrm{X}^{\prime}\left(\mathrm{R}^{-1}-\mathrm{R}^{-1} \mathrm{Z}\left(\mathrm{Z}^{\prime} \mathrm{R}^{-1} \mathrm{Z}+\mathrm{G}^{-1}\right)^{-1} \mathrm{Z}^{\prime} \mathrm{R}^{-1}\right) \mathrm{X} \hat{\alpha} \\
& =\left(\mathrm{X}_{o}^{\prime}-\mathrm{X}^{\prime} \mathrm{R}^{-1} \mathrm{Z}\left(\mathrm{Z}^{\prime} \mathrm{R}^{-1} \mathrm{Z}+\mathrm{G}^{-1}\right)^{-1} \mathrm{Z}_{o}^{\prime}\right) \mathrm{R}_{o}^{-1} \mathrm{y}_{o} \\
& +\left(\mathrm{X}_{a}^{\prime}-\mathrm{X}^{\prime} \mathrm{R}^{-1} \mathrm{Z}\left(\mathrm{Z}^{\prime} \mathrm{R}^{-1} \mathrm{Z}+\mathrm{G}^{-1}\right)^{-1} \mathrm{Z}_{a}^{\prime}\right) \mathrm{R}_{a}^{-1} \hat{\mathrm{y}}_{a}
\end{aligned}
$$

mas, usando o fato que (ver Rao (1973) e apêndice (A.4)),

$$
\mathrm{W}^{-1}=\left(\mathrm{R}+\mathrm{ZGZ} \mathrm{Z}^{\prime}\right)^{-1}=\mathrm{R}^{-1}-\mathrm{R}^{-1} \mathrm{Z}\left(\mathrm{Z}^{\prime} \mathrm{R}^{-1} \mathrm{Z}+\mathrm{G}^{-1}\right)^{-1} \mathrm{Z}^{\prime} \mathrm{R}^{-1}
$$

temos:

$$
\begin{aligned}
\hat{\alpha}= & \left(X^{\prime} W^{-1} X\right)^{-1}\left(X_{o}^{\prime}-X^{\prime} R^{-1} Z\left(Z^{\prime} R^{-1} Z+G^{-1}\right)^{-1} Z_{o}^{\prime}\right) R_{o}^{-1} y_{o} \\
& +\left(X^{\prime} W^{-1} X\right)^{-1}\left(X_{a}^{\prime}-X^{\prime} R^{-1} Z\left(Z^{\prime} R^{-1} Z+G^{-1}\right)^{-1} Z_{a}^{\prime}\right) R_{a}^{-1} \hat{y}_{a}
\end{aligned}
$$


ou definindo $\hat{\mathrm{y}}=\left(\begin{array}{c}\mathrm{y}_{o} \\ \hat{\mathrm{y}}_{a}\end{array}\right)$

$$
\hat{\alpha}=\left(\mathrm{X}^{\prime} \mathrm{T}^{-1} \mathrm{X}\right)^{-1} \mathrm{X}^{\prime} \mathrm{W}^{-1} \hat{\mathrm{y}}
$$

Substituindo (2.16) em (2.14) temos

$$
\begin{aligned}
\hat{\mathrm{b}}= & \left(\mathrm{Z}^{\prime} \mathrm{R}^{-1} \mathrm{Z}+\mathrm{G}^{-1}\right)^{-1}\left[\mathrm{Z}_{o}^{\prime}-\mathrm{Z}^{\prime} \mathrm{R}^{-1} \mathrm{X}\left(\mathrm{X}^{\prime} \mathrm{W}^{-1} \mathrm{X}\right)^{-1}\left(\mathrm{X}_{o}^{\prime}-\mathrm{X}^{\prime} \mathrm{R}^{-1} \mathrm{Z}\left(\mathrm{Z}^{\prime} \mathrm{R}^{-1} \mathrm{Z}\right.\right.\right. \\
& \left.\left.\left.+\mathrm{G}^{-1}\right)^{-1} \mathrm{Z}_{o}^{\prime}\right)\right] \mathrm{R}_{o}^{-1} \mathrm{Y}_{o}+\left(\mathrm{Z}^{\prime} \mathrm{R}^{-1} \mathrm{Z}+\mathrm{G}^{-1}\right)^{-1}\left[\mathrm{Z}_{a}^{\prime}-\mathrm{Z}^{\prime} \mathrm{R}^{-1} \mathrm{X}\left(\mathrm{X}^{\prime} \mathrm{W}^{-1} \mathrm{X}\right)^{-1}\right. \\
& \left.\cdot\left(\mathrm{X}_{a}^{\prime}-\mathrm{X}^{\prime} \mathrm{R}^{-1} \mathrm{Z}\left(\mathrm{Z}^{\prime} \mathrm{R}^{-1} \mathrm{Z}+\mathrm{G}^{-1}\right)^{-1} \mathrm{Z}_{a}^{\prime}\right)\right] \mathrm{R}_{a}^{-1} \hat{\mathrm{Y}}_{a}
\end{aligned}
$$

ou

$$
\hat{b}=\left(Z^{\prime} R^{-1} Z+G^{-1}\right)^{-1}\left(Z^{\prime} R^{-1}-Z^{\prime} R^{-1} X\left(X^{\prime} W^{-1} X\right)^{-1} X^{\prime} W^{-1}\right) \hat{y}
$$

ou ainda, usando a igualdade dada por (2.20) abaixo (ver Rao (1973) e apêndice (A.4))

$$
\begin{gathered}
A=\left(Z^{\prime} R^{-1} Z+G^{-1}\right)^{-1}=G\left(I_{q}-Z^{\prime}\left(Z G Z^{\prime}+R\right)^{-1} Z G\right) \\
\hat{b}=G\left(I_{q}-Z^{\prime} W^{-1} Z G\right) Z^{\prime} R^{-1}\left[I_{d}-X\left(X^{\prime} W^{-1} X\right)^{-1} X^{\prime} W^{-1}\right] \hat{y}
\end{gathered}
$$

Agora, usando (2.13), (2.16), (2.18) e (2.20), temos que

$$
\begin{aligned}
\hat{y}_{a}= & \left\{I_{m}-X_{a}\left(X^{\prime} W^{-1} X\right)^{-1}\left[X_{a}^{\prime}-X^{\prime} R^{-1} Z A Z_{a}^{\prime}\right] R_{a}^{-1}-Z_{a} A\left[Z_{a}^{\prime}-Z^{\prime} R^{-1} X\left(X^{\prime} W^{-1} X\right)^{-1}\right.\right. \\
& \left.\left.\cdot\left(X_{a}^{\prime}-X^{\prime} R^{-1} Z A Z_{a}^{\prime}\right)\right] R_{a}^{-1}\right\}^{-1}\left\{X_{a}\left(X^{\prime} W^{-1} X\right)^{-1}\left[X_{o}^{\prime}-X^{\prime} R^{-1} Z A Z_{o}^{\prime}\right]\right. \\
& \left.+Z_{a} A\left[Z_{o}^{\prime}-Z^{\prime} R^{-1} X\left(X^{\prime} W^{-1} X\right)^{-1}\left(X_{o}^{\prime}-X^{\prime} R^{-1} Z A Z_{o}^{\prime}\right)\right]\right\} R_{o}^{-1} y_{o}
\end{aligned}
$$


Note que os previsores de $\alpha(2.16)$ e de b (2.18) são funções do previsor (2.22) da observação perdida $\mathrm{y}_{a}$. Isto sugere primeiro prevermos $\mathrm{y}_{a}$, completando os dados, e depois prevermos $\alpha$ e b com os dados completados. Note ainda que as expressões (2.17) e (2.19), dos previsores de $\alpha$ e b , são as mesmas obtidas para dados completos (expressões (2.5) e (2.6)), só que aplicada aos dados completados.

O caminho inverso é equivalente, isto é, poderíamos prever $\alpha$ e b usando os dados incompletos e depois, prever $\mathrm{y}_{a}$ através da combinação linear dos previsores de $\alpha$ e b. Esta maneira, apesar de mais intuitiva, nem sempre é a melhor, pois para prever $\alpha$ e b teríamos que inverter a matriz de covariâncias dos dados incompletos, o que às vezes é bastante complicado.

Teorema 2.1: Suponha o modelo dado por (2.1) com m observaçôes perdidas. Os previsores lineares não-viesados de erro quadrático médio mínimo (BLUP) de $\boldsymbol{\alpha}, \mathrm{b}$ e $\mathrm{y}_{a}$ são dados pelas expressões (2.16), (2.18) e (2.22), respectivamente.

\section{Prova}

\section{i. Linear}

Que os previsores são lineares é óbvio pois todos são da forma $l^{\prime} \mathrm{y}_{0}$. 
ii. Não-viesado

Seja

$$
\begin{aligned}
\mathrm{D}= & \mathrm{I}_{m}-\mathrm{X}_{a}\left(\mathrm{X}^{\prime} \mathrm{W}^{-1} \mathrm{X}\right)^{-1}\left(\mathrm{X}_{a}^{\prime}-\mathrm{X}^{\prime} \mathrm{R}^{-1} \mathrm{ZAZ}_{a}^{\prime}\right) \mathrm{R}_{a}^{-1}-\mathrm{Z}_{a} \mathrm{~A}\left[\mathrm{Z}_{a}^{\prime}\right. \\
& \left.-\mathrm{Z}^{\prime} \mathrm{R}^{-1} \mathrm{X}\left(\mathrm{X}^{\prime} \mathrm{W}^{-1} \mathrm{X}\right)^{-1}\left(\mathrm{X}_{a}^{\prime}-\mathrm{X}^{\prime} \mathrm{R}^{-1} \mathrm{ZAZ}_{a}^{\prime}\right)\right] \mathrm{R}_{a}^{-1}
\end{aligned}
$$

De (2.22) temos

$$
\begin{aligned}
\mathbb{E}\left(\hat{\mathrm{y}}_{a}\right) & =\mathrm{D}^{-1}\left\{\mathrm{X}_{a}\left(\mathrm{X}^{\prime} \mathrm{W}^{-1} \mathrm{X}\right)^{-1}\left[\mathrm{X}_{o}^{\prime}-\mathrm{X}^{\prime} \mathrm{R}^{-1} \mathrm{ZAZ}_{o}^{\prime}\right]+\mathrm{Z}_{a} \mathrm{~A}\left[\mathrm{Z}_{o}^{\prime}-\mathrm{Z}^{\prime} \mathrm{R}^{-1} \mathrm{X}\right.\right. \\
& \left.\left.\cdot\left(\mathrm{X}^{\prime} \mathrm{W}^{-1} \mathrm{X}\right)^{-1}\left(\mathrm{X}_{o}^{\prime}-\mathrm{X}^{\prime} \mathrm{R}^{-1} \mathrm{Z} \mathrm{AZ} \mathrm{Z}_{o}^{\prime}\right)\right]\right\} \mathrm{R}_{o}^{-1} \mathrm{X}_{\circ} \alpha \\
& =\mathrm{D}^{-1}\left\{\mathrm{X}_{a}\left(\mathrm{X}^{\prime} \mathrm{W}^{-1} \mathrm{X}\right)^{-1}\left(\mathrm{X}_{o}^{\prime} \mathrm{R}_{o}^{-1} \mathrm{X}_{o}-\mathrm{X}^{\prime} \mathrm{R}^{-1} \mathrm{Z} \mathrm{A} \mathrm{Z}_{o}^{\prime} \mathrm{R}_{o}^{-1} \mathrm{X}_{o}\right)+\mathrm{Z}_{a} \mathrm{~A}\left[\mathrm{Z}_{o}^{\prime}\right.\right. \\
& \left.\left.\cdot \mathrm{R}_{o}^{-1} \mathrm{X}_{o}-\mathrm{Z}^{\prime} \mathrm{R}^{-1} \mathrm{X}\left(\mathrm{X}^{\prime} \mathrm{W}^{-1} \mathrm{X}\right)^{-1}\left(\mathrm{X}_{o}^{\prime} \mathrm{R}_{o}^{-1} \mathrm{X}_{o}-\mathrm{X}^{\prime} \mathrm{R}^{-1} \mathrm{ZA} \mathrm{Z}_{o}^{\prime} \mathrm{R}_{o}^{-1} \mathrm{X}_{o}\right)\right]\right\} \alpha
\end{aligned}
$$

Mas $\mathrm{X}_{o}^{\prime} \mathbf{R}_{o}^{-1} \mathbf{X}_{o}=\mathrm{X}^{\prime} \mathrm{R}^{-1} \mathrm{X}-\mathrm{X}_{a}^{\prime} \mathrm{R}_{a}^{-1} \mathbf{X}_{a}$ e $\mathrm{Z}_{o}^{\prime} \mathbf{R}_{o}^{-1} \mathbf{X}_{o}=\mathrm{Z}^{\prime} \mathbf{R}^{-1} \mathrm{X}-\mathbf{Z}_{a}^{\prime} \mathbf{R}_{a}^{-1} \mathbf{X}_{a}$ e então

$$
\begin{aligned}
& \mathbb{E}\left(\hat{\mathrm{y}}_{a}\right)=\mathrm{D}^{-1}\left\{\mathrm { X } _ { a } ( \mathrm { X } ^ { \prime } \mathrm { W } ^ { - 1 } \mathrm { X } ) ^ { - 1 } \left(\mathrm{X}^{\prime} \mathrm{R}^{-1} \mathrm{X}-\mathrm{X}_{a}^{\prime} \mathrm{R}_{a}^{-1} \mathrm{X}_{a}-\mathrm{X}^{\prime} \mathrm{R}^{-1} \mathrm{Z} \mathrm{AZ}^{\prime} \mathrm{R}^{-1} \mathrm{X}\right.\right. \\
& \left.+\mathrm{X}^{\prime} \mathrm{R}^{-1} \mathrm{ZA} \mathrm{Z}_{a}^{\prime} \mathrm{R}_{a}^{-1} \mathrm{X}_{a}\right)+\mathrm{Z}_{a} \mathrm{~A}\left[\mathrm{Z}^{\prime} \mathrm{R}^{-1} \mathrm{X}-\mathrm{Z}_{a}^{\prime} \mathrm{R}_{a}^{-1} \mathrm{X}_{a}-\mathrm{Z}^{\prime} \mathrm{R}^{-1} \mathrm{X}\left(\mathrm{X}^{\prime} \mathrm{W}^{-1} \mathrm{X}\right)^{-1}\right. \\
& \left.\left.\cdot\left(\mathrm{X}^{\prime} \mathrm{R}^{-1} \mathrm{X}-\mathrm{X}_{a}^{\prime} \mathrm{R}_{a}^{-1} \mathrm{X}_{a}-\mathrm{X}^{\prime} \mathrm{R}^{-1} \mathrm{ZAZ}^{\prime} \mathrm{R}^{-1} \mathrm{X}+\mathrm{X}^{\prime} \mathrm{R}^{-1} \mathrm{ZAZ}_{a}^{\prime} \mathrm{R}_{a}^{-1} \mathrm{X}_{a}\right)\right]\right\} \alpha \\
& =\mathrm{D}^{-1}\left\{\mathrm { X } _ { a } ( \mathrm { X } ^ { \prime } \mathrm { W } ^ { - 1 } \mathrm { X } ) ^ { - 1 } \left(\mathrm{X}^{\prime}\left(\mathrm{R}^{-1}-\mathrm{R}^{-1} \mathrm{ZAZ}^{\prime} \mathrm{R}^{-1}\right) \mathrm{X}-\mathrm{X}_{a}^{\prime} \mathrm{R}_{a}^{-1} \mathrm{X}_{a}\right.\right. \\
& \left.+\mathrm{X}^{\prime} \mathrm{R}^{-1} \mathrm{ZAZ}_{a}^{\prime} \mathrm{R}_{a}^{-1} \mathrm{X}_{a}\right)+\mathrm{Z}_{a} \mathrm{~A}\left(\mathrm { Z } ^ { \prime } \left(\mathrm{R}^{-1} \mathrm{X}-\mathrm{R}^{-1} \mathrm{X}\left(\mathrm{X}^{\prime} \mathrm{W}^{-1} \mathrm{X}\right)^{-1}\left(\mathrm{X}^{\prime} \mathrm{R}^{-1} \mathrm{X}\right.\right.\right. \\
& \left.\left.-\mathrm{X}^{\prime} \mathrm{R}^{-1} \mathrm{ZAZ} \mathrm{Z}^{\prime} \mathrm{R}^{-1} \mathrm{X}\right)\right)-\mathrm{Z}_{a}^{\prime} \mathrm{R}_{a}^{-1} \mathrm{X}_{a}+\mathrm{Z}^{\prime} \mathrm{R}^{-1} \mathrm{X}\left(\mathrm{X}^{\prime} \mathrm{W}^{-1} \mathrm{X}\right)^{-1} \\
& \left.\left.\cdot \mathrm{X}_{a}^{\prime} \mathrm{R}_{a}^{-1} \mathrm{X}_{a}-\mathrm{Z}^{\prime} \mathrm{R}^{-1} \mathrm{X}\left(\mathrm{X}^{\prime} \mathrm{W}^{-1} \mathrm{X}\right)^{-1} \mathrm{X}^{\prime} \mathrm{R}^{-1} \mathrm{ZAZ}_{a}^{\prime} \mathrm{R}_{a}^{-1} \mathrm{X}_{a}\right)\right\} \alpha
\end{aligned}
$$


Usando a expressão (2.20), ou seja, $A=\left(Z^{\prime} R^{-1} Z+G^{-1}\right)^{-1}$, temos da expressão (2.15) que $\mathrm{W}^{-1}=\mathrm{R}^{-1}-\mathrm{R}^{-1} \mathrm{ZAZ}^{\prime} \mathrm{R}^{-1}$ e assim

$$
\begin{aligned}
& \mathbb{E}\left(\hat{\mathrm{y}}_{a}\right)=\mathrm{D}^{-1}\left\{\mathrm{X}_{a}\left(\mathrm{X}^{\prime} \mathrm{W}^{-1} \mathrm{X}\right)^{-1}\left[\mathrm{X}^{\prime} \mathrm{W}^{-1} \mathrm{X}-\mathrm{X}_{a}^{\prime} \mathrm{R}_{a}^{-1} \mathrm{X}_{a}+\mathrm{X}^{\prime} \mathrm{R}^{-1} \mathrm{ZAZ}_{a}^{\prime} \mathrm{R}_{a}^{-1} \mathrm{X}_{a}\right]\right. \\
& +\mathrm{Z}_{a} \mathrm{~A}\left[\mathrm{Z}^{\prime} \mathrm{R}^{-1}\left(\mathrm{X}-\mathrm{X}\left(\mathrm{X}^{\prime} \mathrm{W}^{-1} \mathrm{X}\right)^{-1} \mathrm{X}^{\prime} \mathrm{W}^{-1} \mathrm{X}\right)-\left(\mathrm{Z}_{a}^{\prime}-\mathrm{Z}^{\prime} \mathrm{R}^{-1} \mathrm{X}\left(\mathrm{X}^{\prime} \mathrm{W}^{-1} \mathrm{X}\right)^{-1} \mathrm{X}_{a}^{\prime}\right.\right. \\
& \left.\left.\left.+\mathrm{Z}^{\prime} \mathrm{R}^{-1} \mathrm{X}\left(\mathrm{X}^{\prime} \mathrm{W}^{-1} \mathrm{X}\right)^{-1} \mathrm{X}^{\prime} \mathrm{R}^{-1} \mathrm{ZAZ}_{a}^{\prime}\right) \mathrm{R}_{a}^{-1} \mathrm{X}_{a}\right]\right\} \alpha \\
& =\mathrm{D}^{-1}\left\{\mathrm{X}_{a}-\mathrm{X}_{a}\left(\mathrm{X}^{\prime} \mathrm{W}^{-1} \mathrm{X}\right)^{-1} \mathrm{X}_{a}^{\prime} \mathrm{R}_{a}^{-1} \mathrm{X}_{a}+\mathrm{X}_{a}\left(\mathrm{X}^{\prime} \mathrm{W}^{-1} \mathrm{X}\right)^{-1} \mathrm{X}^{\prime} \mathrm{R}^{-1} \mathrm{ZAZ}_{a}^{\prime} \mathrm{R}_{a}^{-1} \mathrm{X}_{a}\right. \\
& \left.+\mathrm{Z}_{a} \mathrm{~A}\left[-\mathrm{Z}_{a}^{\prime}+\mathrm{Z}^{\prime} \mathrm{R}^{-1} \mathrm{X}\left(\mathrm{X}^{\prime} \mathrm{W}^{-1} \mathrm{X}\right)^{-1}\left(\mathrm{X}_{a}^{\prime}-\mathrm{X}^{\prime} \mathrm{R}^{-1} \mathrm{ZAZ}_{a}^{\prime}\right)\right] \mathrm{R}_{a}^{-1} \mathrm{X}_{a}\right\} \alpha \\
& =\mathrm{D}^{-1}\left\{\mathrm{I}_{m}-\mathrm{X}_{a}\left(\mathrm{X}^{\prime} \mathrm{W}^{-1} \mathrm{X}\right)^{-1}\left(\mathrm{X}_{a}^{\prime}-\mathrm{X}^{\prime} \mathrm{R}^{-1} \mathrm{Z} \mathrm{A} \mathrm{Z}_{a}^{\prime}\right) \mathrm{R}_{a}^{-1}-\mathrm{Z}_{a} \mathrm{~A}\left[\mathrm{Z}_{a}^{\prime}-\mathrm{Z}^{\prime} \mathrm{R}^{-1} \mathrm{X}\right.\right. \\
& \left.\left.\cdot\left(\mathrm{X}^{\prime} \mathrm{W}^{-1} \mathrm{X}\right)^{-1}\left(\mathrm{X}_{a}^{\prime}-\mathrm{X}^{\prime} \mathrm{R}^{-1} \mathrm{ZAZ}_{a}^{\prime}\right)\right] \mathrm{R}_{a}^{-1}\right\} \mathrm{X}_{a} \alpha \\
& =\mathrm{D}^{-1} \mathrm{DX}_{a} \alpha=\mathrm{X}_{a} \alpha=\mathbb{E}\left(\mathrm{y}_{a}\right)
\end{aligned}
$$

Portanto, $\hat{\mathbf{y}}_{a}$ é não-viesado para $\mathrm{y}_{a}$.

Partindo da expressão (2.17), tem-se que

$$
\begin{aligned}
\mathbb{E}(\hat{\alpha}) & =\left(\mathrm{X}^{\prime} \mathrm{W}^{-1} \mathrm{X}\right)^{-1} \mathrm{X}^{\prime} \mathrm{W}^{-1} \mathbb{E}(\hat{\mathrm{y}}) \\
& =\left(\mathrm{X}^{\prime} \mathrm{W}^{-1} \mathrm{X}\right)^{-1} \mathrm{X}^{\prime} \mathrm{W}^{-1} \mathbb{E}\left(\begin{array}{c}
\mathrm{y}_{o} \\
\hat{\mathrm{y}}_{a}
\end{array}\right) \\
& =\left(\mathrm{X}^{\prime} \mathrm{W}^{-1} \mathrm{X}\right)^{-1} \mathrm{X}^{\prime} \mathrm{W}^{-1}\left(\begin{array}{c}
\mathrm{X}_{o} \\
\mathrm{X}_{a}
\end{array}\right) \alpha \\
& =\left(\mathrm{X}^{\prime} \mathrm{W}^{-1} \mathrm{X}\right)^{-1} \mathrm{X}^{\prime} \mathrm{W}^{-1} \mathrm{X} \alpha=\alpha .
\end{aligned}
$$

Logo, $\hat{\alpha}$ é não-viesado para $\alpha$. 
Partindo agora de (2.19),

$$
\begin{aligned}
\mathbb{E}(\hat{\mathrm{b}}) & =\left(\mathrm{Z}^{\prime} \mathrm{R}^{-1} \mathrm{Z}+\mathrm{G}^{-1}\right)^{-1}\left(\mathrm{Z}^{\prime} \mathrm{R}^{-1}-\mathrm{Z}^{\prime} \mathrm{R}^{-1} \mathrm{X}\left(\mathrm{X}^{\prime} \mathrm{W}^{-1} \mathrm{X}\right)^{-1} \mathrm{X}^{\prime} \mathrm{W}^{-1}\right) \mathbb{E}(\hat{\mathrm{y}}) \\
& =\left(\mathrm{Z}^{\prime} \mathrm{R}^{-1} \mathrm{Z}+\mathrm{G}^{-1}\right)^{-1}\left(\mathrm{Z}^{\prime} \mathrm{R}^{-1}-\mathrm{Z}^{\prime} \mathrm{R}^{-1} \mathrm{X}\left(\mathrm{X}^{\prime} \mathrm{W}^{-1} \mathrm{X}\right)^{-1} \mathrm{X}^{\prime} \mathrm{W}^{-1}\right)\left(\begin{array}{c}
\mathrm{X}_{\circ} \\
\mathrm{X}_{a}
\end{array}\right) \alpha \\
& =\left(\mathrm{Z}^{\prime} \mathrm{R}^{-1} \mathrm{Z}+\mathrm{G}^{-1}\right)^{-1}\left(\mathrm{Z}^{\prime} \mathrm{R}^{-1}-\mathrm{Z}^{\prime} \mathrm{R}^{-1} \mathrm{X}\left(\mathrm{X}^{\prime} \mathrm{W}^{-1} \mathrm{X}\right)^{-1} \mathrm{X}^{\prime} \mathrm{W}^{-1}\right) \mathrm{X} \alpha \\
& =\left(\mathrm{Z}^{\prime} \mathrm{R}^{-1} \mathrm{Z}+\mathrm{G}^{-1}\right)^{-1}\left(\mathrm{Z}^{\prime} \mathrm{R}^{-1} \mathrm{X}-\mathrm{Z}^{\prime} \mathrm{R}^{-1} \mathrm{X}\right) \alpha \\
& =0=\mathbb{E}(\mathrm{b})
\end{aligned}
$$

Assim, $\hat{b}$ é não-viesado para b.

\section{iii. EQM mínimo}

Primeiro, note que:

$$
\begin{aligned}
& \mathbb{E}\left(\mathrm{y}_{o} \mathrm{y}_{o}^{\prime}\right)=\mathrm{X}_{o} \alpha \alpha^{\prime} \mathrm{X}_{o}^{\prime}+\mathrm{Z}_{o} \mathbb{E}\left(\mathrm{bb}^{\prime}\right) \mathrm{Z}_{o}^{\prime}+\mathbb{E}\left(\mathrm{e}_{o} \mathrm{e}_{o}^{\prime}\right)=\mathrm{X}_{o} \alpha \alpha^{\prime} \mathrm{X}_{o}^{\prime}+\mathrm{Z}_{o} \mathrm{GZ} \mathrm{Z}_{o}^{\prime} \sigma^{2}+\mathrm{R}_{o} \sigma^{2} \\
& \mathbb{E}\left(\mathrm{y}_{a} \mathrm{y}_{o}^{\prime}\right)=\mathrm{X}_{a} \alpha \alpha^{\prime} \mathrm{X}_{o}^{\prime}+\mathrm{Z}_{a} \mathbb{E}\left(\mathrm{bb}^{\prime}\right) \mathrm{Z}_{o}^{\prime}+\mathbb{E}\left(\mathrm{e}_{a} \mathrm{e}_{o}^{\prime}\right)=\mathrm{X}_{a} \alpha \alpha^{\prime} \mathrm{X}_{o}^{\prime}+\mathrm{Z}_{a} \mathrm{GZ}_{o}^{\prime} \sigma^{2}
\end{aligned}
$$

e

$$
\mathbb{E}\left(\mathrm{by}_{o}^{\prime}\right)=\mathbb{E}\left(\mathrm{bb}^{\prime}\right) \mathrm{Z}_{o}^{\prime}=\mathrm{GZ}_{o}^{\prime} \sigma^{2}
$$

Note também que quaisquer previsores lineares de $\alpha, \mathrm{b}$ e $\mathrm{y}_{a}$ são da forma $\hat{\alpha}+\mathrm{l}^{\prime} \mathrm{y}_{o}$, $\hat{\mathrm{b}}+\mathrm{l}^{\prime} \mathrm{y}_{o}$ e $\hat{\mathrm{y}}_{a}+\mathrm{l}^{\prime} \mathrm{y}_{o}$, respectivamente e, para que sejam não-viesados é necessário que $\mathrm{X}_{0}^{\prime} \mathrm{l}=0$ ( l'yó um estimador não-viesado de zero).

$\mathrm{O}$ erro quadrático médio de qualquer previsor linear não-viesado de $\mathrm{y}_{a}$ é dado por:

$$
\begin{aligned}
& \mathbb{E}\left[\left(\hat{\mathrm{y}}_{a}+\mathrm{l}^{\prime} \mathrm{y}_{o}-\mathrm{y}_{a}\right)\left(\hat{\mathrm{y}}_{a}+\mathrm{l}^{\prime} \mathrm{y}_{o}-\mathrm{y}_{a}\right)^{\prime}\right] \\
& \quad=\mathbb{E}\left[\left(\hat{\mathrm{y}}_{a}-\mathrm{y}_{a}\right)\left(\hat{\mathrm{y}}_{a}-\mathrm{y}_{a}\right)^{\prime}\right]+\mathbb{E}\left[\left(\hat{\mathrm{y}}_{a}-\mathrm{y}_{a}\right) \mathrm{y}_{o}^{\prime} \mathrm{l}\right]+\mathbb{E}\left[\mathrm{l}^{\prime} \mathrm{y}_{o}\left(\hat{\mathrm{y}}_{a}-\mathrm{y}_{a}\right)^{\prime}\right]+\mathbb{E}\left[\mathrm{l}^{\prime} \mathrm{y}_{o} \mathrm{y}_{o}^{\prime} \mathrm{l}\right] .
\end{aligned}
$$


Agora, vamos mostrar que $\mathbb{E}\left[\left(\hat{\mathrm{y}}_{a}-\mathrm{y}_{a}\right) \mathrm{y}_{o}^{\prime} 1\right]=0$.

Note que

$$
\mathbb{E}\left[\left(\hat{\mathrm{y}}_{a}-\mathrm{y}_{a}\right) \mathrm{y}_{o}^{\prime} \mathrm{l}\right]=\mathbb{E}\left[\left(\mathrm{D}^{-1} \mathrm{By}_{\circ}-\mathrm{y}_{a}\right) \mathrm{y}_{o}^{\prime} \mathrm{l}\right],
$$

onde $\mathrm{D}$ é dado por (2.23) e

$$
\begin{aligned}
& \mathbf{B}=\left[\mathrm{X}_{a}\left(\mathrm{X}^{\prime} \mathbf{W}^{-1} \mathrm{X}\right)^{-1}\left(\mathrm{X}_{o}^{\prime}-\mathrm{X}^{\prime} \mathbf{R}^{-1} \mathrm{ZAZ} \mathrm{Z}_{o}^{\prime}\right)+\mathrm{Z}_{a} \mathrm{~A}\left(\mathrm{Z}_{o}^{\prime}-\mathrm{Z}^{\prime} \mathrm{R}^{-1} \mathrm{X}\left(\mathrm{X}^{\prime} \mathbf{W}^{-1} \mathrm{X}\right)^{-1}\right.\right. \\
& \left.\left.\cdot\left(\mathrm{X}_{o}^{\prime}-\mathrm{X}^{\prime} \mathrm{R}^{-1} \mathrm{ZAZ} \mathrm{Z}_{o}^{\prime}\right)\right)\right] \mathrm{R}_{o}^{-1} \\
& \mathbb{E}\left[\left(\hat{\mathrm{y}}_{a}-\mathrm{y}_{a}\right) \mathrm{y}_{o}^{\prime} \mathrm{l}\right]=\mathrm{D}^{-1} \mathrm{~B} \mathbb{E}\left(\mathrm{y}_{o} \mathrm{y}_{o}^{\prime}\right) \mathrm{l}-\mathbb{E}\left(\mathrm{y}_{a} \mathrm{y}_{o}^{\prime}\right) \mathrm{l} \\
& =\mathrm{D}^{-1} \mathrm{~B}\left[\mathrm{X}_{\circ} \alpha \alpha^{\prime} \mathrm{X}_{\circ}^{\prime}+\mathrm{Z}_{\circ} \mathrm{GZ}_{\circ}^{\prime} \sigma^{2}+\mathrm{R}_{o} \sigma^{2}\right] 1-\left[\mathrm{X}_{a} \alpha \alpha^{\prime} \mathrm{X}_{o}^{\prime}+\mathrm{Z}_{a} \mathrm{GZ}_{o}^{\prime} \sigma^{2}\right] 1 \text {. } \\
& \text { Como } \mathrm{X}_{0}^{\prime} \mathrm{l}=0 \text {, } \\
& \mathbb{E}\left[\left(\hat{\mathrm{y}}_{a}-\mathrm{y}_{a}\right) \mathrm{y}_{0}^{\prime} \mathrm{l}\right] \\
& =\mathrm{D}^{-1}\left[\left(\mathrm{BZ}_{o}-\mathrm{DZ}_{a}\right) \mathrm{GZ}_{\circ}^{\prime}+\mathrm{BR}_{o}\right] l \sigma^{2} \\
& =\mathrm{D}^{-1}\left\{\left[\mathrm{X}_{a}\left(\mathrm{X}^{\prime} \mathrm{W}^{-1} \mathrm{X}\right)^{-1}\left(\mathrm{X}_{o}^{\prime} \mathrm{R}_{o}^{-1} \mathrm{Z}_{o}-\mathrm{X}^{\prime} \mathrm{R}^{-1} \mathrm{ZAZ}_{o}^{\prime} \mathrm{R}_{o}^{-1} \mathrm{Z}_{o}\right)+\mathrm{Z}_{a} \mathrm{~A}\left(\mathrm{Z}_{o}^{\prime} \mathrm{R}_{o}^{-1} \mathrm{Z}_{o}\right.\right.\right. \\
& \left.\left.-\mathrm{Z}^{\prime} \mathrm{R}^{-1} \mathrm{X}\left(\mathrm{X}^{\prime} \mathrm{W}^{-1} \mathrm{X}\right)^{-1}\left(\mathrm{X}_{\circ}^{\prime} \mathrm{R}_{o}^{-1} \mathrm{Z}_{o}-\mathrm{X}^{\prime} \mathrm{R}^{-1} \mathrm{ZAZ}_{o}^{\prime} \mathrm{R}_{o}^{-1} \mathrm{Z}_{o}\right)\right)\right] \mathrm{GZ} \mathrm{Z}_{o}^{\prime} \mathrm{l} \sigma^{2} \\
& +\left[-\mathrm{Z}_{a}+\mathrm{X}_{a}\left(\mathrm{X}^{\prime} \mathrm{W}^{-1} \mathrm{X}\right)^{-1}\left(\mathrm{X}_{a}^{\prime} \mathrm{R}_{a}^{-1} \mathrm{Z}_{a}-\mathrm{X}^{\prime} \mathrm{R}^{-1} \mathrm{ZAZ}_{a}^{\prime} \mathrm{R}_{a}^{-1} \mathrm{Z}_{a}\right)+\mathrm{Z}_{a} \mathrm{~A}\left(\mathrm{Z}_{a}^{\prime} \mathrm{R}_{a}^{-1} \mathrm{Z}_{a}\right.\right. \\
& \left.\left.-\mathrm{Z}^{\prime} \mathrm{R}^{-1} \mathrm{X}\left(\mathrm{X}^{\prime} \mathrm{W}^{-1} \mathrm{X}\right)^{-1}\left(\mathrm{X}_{a}^{\prime} \mathrm{R}_{a}^{-1} \mathrm{Z}_{a}-\mathrm{X}^{\prime} \mathrm{R}^{-1} \mathrm{ZAZ}_{a}^{\prime} \mathrm{R}_{a}^{-1} \mathrm{Z}_{a}\right)\right)\right] \mathrm{G} \mathrm{Z}_{o}^{\prime} l \sigma^{2}-\mathrm{X}_{a}\left(\mathrm{X}^{\prime} \mathrm{W}^{-1} \mathrm{X}\right)^{-1} \\
& \left.\cdot \mathbf{X}^{\prime} \mathbf{R}^{-1} \mathbf{Z} \mathbf{A} Z_{o}^{\prime} l \sigma^{2}+\mathbf{Z}_{a} \mathbf{A}\left(\mathbf{Z}_{o}^{\prime} l \sigma^{2}+\mathbf{Z}^{\prime} \mathbf{R}^{-1} \mathbf{X}\left(\mathbf{X}^{\prime} \mathbf{W}^{-1} \mathbf{X}\right)^{-1} \mathbf{X}^{\prime} \mathbf{R}^{-1} \mathbf{Z} \mathbf{A} \mathbf{Z}_{o}^{\prime} l \sigma^{2}\right)\right\}
\end{aligned}
$$




$$
\begin{aligned}
& =\mathrm{D}^{-1}\left\{\left[\mathrm{X}_{a}\left(\mathrm{X}^{\prime} \mathrm{W}^{-1} \mathrm{X}\right)^{-1}\left(\mathrm{X}^{\prime} \mathrm{R}^{-1} \mathrm{Z}-\mathrm{X}^{\prime} \mathrm{R}^{-1} \mathrm{ZA} \mathrm{Z}^{\prime} \mathrm{R}^{-1} \mathrm{Z}\right)+\mathrm{Z}_{a} \mathrm{~A}\left(\mathrm{Z}^{\prime} \mathrm{R}^{-1} \mathrm{Z}-\mathrm{Z}^{\prime} \mathrm{R}^{-1} \mathrm{X}\right.\right.\right. \\
& \left.\left.\cdot\left(\mathrm{X}^{\prime} \mathrm{W}^{-1} \mathrm{X}\right)^{-1}\left(\mathrm{X}^{\prime} \mathrm{R}^{-1} \mathrm{Z}-\mathrm{X}^{\prime} \mathrm{R}^{-1} \mathrm{ZAZ}^{\prime} \mathrm{R}^{-1} \mathrm{Z}\right)\right)\right] \mathrm{GZ} \mathrm{Z}_{0}^{\prime} l \sigma^{2}-\mathrm{Z}_{a} \mathrm{G} \mathrm{Z}_{0}^{\prime} l \sigma^{2}-\mathrm{X}_{a}\left(\mathrm{X}^{\prime} \mathrm{W}^{-1} \mathrm{X}\right)^{-1} \\
& \left.\cdot \mathrm{X}^{\prime} \mathrm{R}^{-1} \mathrm{Z} \mathrm{AZ} \mathbf{Z}_{o}^{\prime} l \sigma^{2}+\mathrm{Z}_{a} \mathrm{~A}\left(\mathrm{Z}_{0}^{\prime} \mathrm{l} \sigma^{2}+\mathrm{Z}^{\prime} \mathrm{R}^{-1} \mathrm{X}\left(\mathrm{X}^{\prime} \mathrm{W}^{-1} \mathrm{X}\right)^{-1} \mathrm{X}^{\prime} \mathrm{R}^{-1} \mathrm{ZAZ} \mathbf{Z}_{o}^{\prime} l \sigma^{2}\right)\right\} \\
& =\mathrm{D}^{-1}\left\{\mathrm{X}_{a}\left(\mathrm{X}^{\prime} \mathrm{W}^{-1} \mathrm{X}\right)^{-1} \mathrm{X}^{\prime} \mathrm{R}^{-1} \mathrm{Z}\left(\mathrm{G}-\mathrm{A} \mathrm{Z}^{\prime} \mathrm{R}^{-1} \mathrm{ZG}-\mathrm{A}\right) \mathrm{Z}_{o}^{\prime} l \sigma^{2}+\mathrm{Z}_{a} \mathrm{~A}\left(\mathrm{Z}^{\prime} \mathrm{R}^{-1} \mathrm{ZG}+\mathrm{I}_{q}\right)\right. \\
& \left.\cdot \mathrm{Z}_{o}^{\prime} l \sigma^{2}-\mathrm{Z}_{a} \mathrm{~A} \mathrm{Z}^{\prime} \mathrm{R}^{-1} \mathrm{X}\left(\mathrm{X}^{\prime} \mathrm{W}^{-1} \mathrm{X}\right)^{-1} \mathrm{X}^{\prime} \mathrm{R}^{-1} \mathrm{Z}\left(\mathrm{G}-\mathrm{A} \mathrm{Z}^{\prime} \mathrm{R}^{-1} \mathrm{ZG}-\mathrm{A}\right) \mathrm{Z}_{o}^{\prime} l \sigma^{2}-\mathrm{Z}_{a} \mathrm{GZ} Z_{o}^{\prime} l \sigma^{2}\right\} \\
& =\mathrm{D}^{-1}\left[\mathrm{X}_{a}\left(\mathrm{X}^{\prime} \mathrm{W}^{-1} \mathrm{X}\right)^{-1} \mathrm{X}^{\prime} \mathrm{R}^{-1} \mathrm{Z}-\mathrm{Z}_{a}-\mathrm{Z}_{a} \mathrm{~A} \mathrm{Z}^{\prime} \mathrm{R}^{-1} \mathrm{X}\left(\mathrm{X}^{\prime} \mathrm{W}^{-1} \mathrm{X}\right)^{-1} \mathrm{X}^{\prime} \mathrm{R}^{-1} \mathrm{Z}\right] \\
& \cdot\left(\mathrm{G}-\mathrm{A} \mathbf{Z}^{\prime} \mathbf{R}^{-1} \mathrm{ZG}-\mathrm{A}\right) \mathrm{Z}_{\circ}^{\prime} l \sigma^{2} \text {. }
\end{aligned}
$$

Mas,

$$
\mathrm{G}-\mathrm{A} \mathrm{Z}^{\prime} \mathrm{R}^{-1} \mathrm{ZG}-\mathrm{A}=\mathrm{G}-\mathrm{A}\left(\mathrm{Z}^{\prime} \mathrm{R}^{-1} \mathrm{ZG}+\mathrm{I}_{q}\right)
$$$$
=G-A\left(Z^{\prime} R^{-1} Z G+G^{-1} G\right)=G-A\left(Z^{\prime} R^{-1} Z+G^{-1}\right) G=G-A A^{-1} G=0
$$

e então

$$
\mathbb{E}\left[\left(\hat{\mathbf{y}}_{a}-\mathrm{y}_{a}\right) \mathrm{y}_{o}^{\prime} \mathrm{l}\right]=0 .
$$

Voltando à expressão (2.26), o erro quadrático médio de qualquer previsor linear nãoviesado de $\mathrm{y}_{a}$ é

$$
\begin{aligned}
& \mathbb{E}\left[\left(\hat{\mathrm{y}}_{a}+\mathrm{l}^{\prime} \mathrm{y}_{o}-\mathrm{y}_{a}\right)\left(\hat{\mathrm{y}}_{a}+\mathrm{l}^{\prime} \mathrm{y}_{o}-\mathrm{y}_{a}\right)^{\prime}\right] \\
= & \mathbb{E}\left[\left(\hat{\mathrm{y}}_{a}-\mathrm{y}_{a}\right)\left(\hat{\mathrm{y}}_{a}-\mathrm{y}_{a}\right)^{\prime}\right]+\mathbb{E}\left[\mathrm{l}^{\prime} \mathrm{y}_{o} \mathrm{y}_{o}^{\prime} \mathrm{l}\right] \geq \mathbb{E}\left[\left(\hat{\mathrm{y}}_{a}-\mathrm{y}_{a}\right)\left(\hat{\mathrm{y}}_{a}-\mathrm{y}_{a}\right)^{\prime}\right]
\end{aligned}
$$

pois $\mathbb{E}\left[\mathrm{l}^{\prime} \mathrm{y}_{o} \mathrm{y}_{o}^{\prime} \mathrm{l}\right]$ é uma matriz definida não-negativa e então $\hat{\mathbf{y}}_{a}$ é o BLUP de $\mathbf{y}_{a}$.

Para mostrar que $\hat{\alpha}$ e b são de erro quadrático médio mínimo, o caminho é análogo, basta verificar que: 


$$
\mathbb{E}\left[(\hat{\alpha}-\alpha) \mathrm{y}_{0}^{\prime} 1\right]=\mathbb{E}\left[\left(\mathrm{L} \mathrm{y}_{0}+\mathrm{H} \hat{y}_{a}-\alpha\right) \mathrm{y}_{o}^{\prime} \mathrm{l}\right]
$$

onde $\mathrm{L}=\left(\mathrm{X}^{\prime} \mathrm{W}^{-1} \mathrm{X}\right)^{-1}\left(\mathrm{X}_{\circ}^{\prime}-\mathrm{X}^{\prime} \mathrm{R}^{-1} \mathrm{Z}\left(\mathrm{Z}^{\prime} \mathrm{R}^{-1} \mathrm{Z}+\mathrm{G}^{-1}\right)^{-1} \mathrm{Z}_{\circ}^{\prime}\right) \mathrm{R}_{o}^{-1} \quad \mathrm{e}$

$$
\mathrm{H}=\left(\mathrm{X}^{\prime} \mathrm{W}^{-1} \mathrm{X}\right)^{-1}\left(\mathrm{X}_{a}^{\prime}-\mathrm{X}^{\prime} \mathrm{R}^{-1} \mathrm{Z}\left(\mathrm{Z}^{\prime} \mathrm{R}^{-1} \mathrm{Z}+\mathrm{G}^{-1}\right)^{-1} \mathrm{Z}_{a}^{\prime}\right) \mathrm{R}_{a}^{-1} .
$$

Assim,

$$
\begin{aligned}
& \mathbb{E}\left[(\hat{\alpha}-\alpha) \mathrm{y}_{0}^{\prime} \mathrm{l}\right]=\mathbb{E}\left[\left(\mathrm{Ly}_{0}+\mathrm{H} \hat{\mathrm{y}}_{a}-\mathrm{Hy}_{a}+\mathrm{Hy}_{a}-\alpha\right) \mathrm{y}_{0}^{\prime} \mathrm{l}\right] \\
& \left.=\mathrm{L} \mathbb{E}\left[\mathrm{y}_{o} \mathrm{y}_{o}^{\prime}\right] 1+\mathrm{H} \mathbb{E}\left[\left(\hat{\mathrm{y}}_{a}-\mathrm{y}_{a}\right) \mathrm{y}_{o}^{\prime} \mathrm{l}\right]+\mathrm{H} \mathbb{E}\left[\mathrm{y}_{a} \mathrm{y}_{o}^{\prime}\right] 1-\mathbb{E}\left[\alpha \mathrm{y}_{o}^{\prime}\right]\right] \\
& =\mathrm{L}\left(\mathrm{X}_{o} \alpha \alpha^{\prime} \mathrm{X}_{o}^{\prime}+\mathrm{Z}_{o} \mathrm{G} Z_{o}^{\prime} \sigma^{2}+\mathrm{R}_{o} \sigma^{2}\right) \mathrm{l}+\mathrm{H}\left(\mathrm{X}_{a} \alpha \alpha^{\prime} \mathrm{X}_{o}^{\prime}+\mathrm{Z}_{a} \mathrm{G} \mathrm{Z}_{o}^{\prime} \sigma^{2}\right) \mathrm{l}-\alpha \alpha^{\prime} \mathrm{X}_{o}^{\prime} \mathrm{l} \\
& =\left(\mathrm{X}^{\prime} \mathrm{W}^{-1} \mathrm{X}\right)^{-1}\left[\mathrm{X}_{\circ}^{\prime} \mathrm{R}_{0}^{-1} \mathrm{Z}_{\circ} \mathrm{G} \mathrm{Z}_{\circ}^{\prime} \sigma^{2}-\mathrm{X}^{\prime} \mathrm{R}^{-1} \mathrm{Z}\left(\mathrm{Z}^{\prime} \mathrm{R}^{-1} \mathrm{Z}+\mathrm{G}^{-1}\right)^{-1} \mathrm{Z}_{\circ}^{\prime} \mathrm{R}_{0}^{-1} \mathrm{Z}_{\circ} \mathrm{G} Z_{\circ}^{\prime} \sigma^{2}\right. \\
& +\mathrm{X}_{o}^{\prime} \sigma^{2}-\mathrm{X}^{\prime} \mathrm{R}^{-1} \mathrm{Z}\left(\mathrm{Z}^{\prime} \mathrm{R}^{-1} \mathrm{Z}+\mathrm{G}^{-1}\right)^{-1} \mathrm{Z}_{o}^{\prime} \sigma^{2} \\
& \left.+\mathrm{X}_{a}^{\prime} \mathrm{R}_{a}^{-1} \mathrm{Z}_{a} \mathrm{GZ} \mathrm{Z}_{o}^{\prime} \sigma^{2}-\mathrm{X}^{\prime} \mathrm{R}^{-1} \mathrm{Z}\left(\mathrm{Z}^{\prime} \mathrm{R}^{-1} \mathrm{Z}+\mathrm{G}^{-1}\right)^{-1} \mathrm{Z}_{a}^{\prime} \mathrm{R}_{a}^{-1} \mathrm{Z}_{a} \mathrm{GZ}_{o}^{\prime} \sigma^{2}\right] \mathrm{l} \\
& =\left(\mathrm{X}^{\prime} \mathrm{W}^{-1} \mathrm{X}\right)^{-1}\left[\mathrm{X}^{\prime} \mathrm{R}^{-1} \mathrm{ZGZ^{ \prime }}-\mathrm{X}^{\prime} \mathrm{R}^{-1} \mathrm{Z}\left(\mathrm{Z}^{\prime} \mathrm{R}^{-1} \mathrm{Z}+\mathrm{G}^{-1}\right)^{-1}\right. \\
& \left.\cdot\left(\mathbf{Z}^{\prime} \mathrm{R}^{-1} \mathrm{ZG}+\mathrm{I}_{q}\right) \mathrm{Z}_{o}^{\prime}\right] \sigma^{2} \mathrm{I} \\
& =\left(\mathrm{X}^{\prime} \mathrm{W}^{-1} \mathrm{X}\right)^{-1}\left(\mathrm{X}^{\prime} \mathrm{R}^{-1} \mathrm{ZGZ}_{\circ}^{\prime}-\mathrm{X}^{\prime} \mathrm{R}^{-1} \mathrm{ZGZ}_{o}^{\prime}\right) \sigma^{2} \mathrm{l}=0
\end{aligned}
$$

e que

$$
\mathbb{E}\left[(\hat{\mathrm{b}}-\mathrm{b}) \mathrm{y}_{0}^{\prime} \mathrm{l}\right]=\mathrm{K} \mathbb{E}\left[\mathrm{y}_{o} \mathrm{y}_{o}^{\prime}\right] \mathrm{l}+\mathrm{M} \mathbb{E}\left[\mathrm{y}_{a} \mathrm{y}_{o}^{\prime}\right] \mathrm{l}-\mathbb{E}\left[\mathrm{by}_{o}^{\prime}\right] \mathrm{l}
$$

onde 


$$
\begin{aligned}
& K=\left(Z^{\prime} R^{-1} Z+G^{-1}\right)^{-1}\left[Z_{o}^{\prime}-Z^{\prime} R^{-1} X\left(X^{\prime} W^{-1} X\right)^{-1}\left(X_{o}^{\prime}-X^{\prime} R^{-1} Z\left(Z^{\prime} R^{-1} Z+G^{-1}\right)^{-1} Z_{o}^{\prime}\right)\right] R_{o}^{-1} \\
& \mathrm{e}=\left(Z^{\prime} R^{-1} Z+G^{-1}\right)^{-1}\left[Z_{a}^{\prime}-Z^{\prime} R^{-1} X\left(X^{\prime} W^{-1} X\right)^{-1}\left(X_{a}^{\prime}-X^{\prime} R^{-1} Z\left(Z^{\prime} R^{-1} Z+G^{-1}\right)^{-1} Z_{a}^{\prime}\right)\right] R_{a}^{-1}
\end{aligned}
$$

e portanto

$$
\begin{aligned}
& \mathbb{E}\left[(\hat{\mathrm{b}}-\mathrm{b}) \mathrm{y}_{o}^{\prime} \mathrm{l}\right] \\
& =\left[( Z ^ { \prime } R ^ { - 1 } Z + G ^ { - 1 } ) ^ { - 1 } \left(Z_{o}^{\prime} R_{o}^{-1} Z_{\circ} G Z_{o}^{\prime}-Z^{\prime} R^{-1} X\left(X^{\prime} W^{-1} X\right)^{-1} X_{o}^{\prime} R_{o}^{-1} Z_{o} G Z_{o}^{\prime}\right.\right. \\
& +\mathrm{Z}^{\prime} \mathrm{R}^{-1} \mathrm{X}\left(\mathrm{X}^{\prime} \mathrm{W}^{-1} \mathrm{X}\right)^{-1} \mathrm{X}^{\prime} \mathrm{R}^{-1} \mathrm{Z}\left(\mathrm{Z}^{\prime} \mathrm{R}^{-1} \mathrm{Z}+\mathrm{G}^{-1}\right)^{-1} \mathrm{Z}_{\circ}^{\prime} \mathrm{R}_{o}^{-1} \mathrm{Z}_{\circ} \mathrm{GZ}_{\circ}^{\prime}+\mathrm{Z}_{\circ}^{\prime} \\
& +\mathrm{Z}^{\prime} \mathrm{R}^{-1} \mathrm{X}\left(\mathrm{X}^{\prime} \mathrm{W}^{-1} \mathrm{X}\right)^{-1} \mathrm{X}^{\prime} \mathrm{R}^{-1} \mathrm{Z}\left(\mathrm{Z}^{\prime} \mathrm{R}^{-1} \mathrm{Z}+\mathrm{G}^{-1}\right)^{-1} \mathrm{Z}^{\prime} \\
& +\mathrm{Z}_{a}^{\prime} \mathrm{R}_{a}^{-1} \mathrm{Z}_{a} \mathrm{G} \mathrm{Z}_{o}^{\prime}-\mathrm{Z}^{\prime} \mathrm{R}^{-1} \mathrm{X}\left(\mathrm{X}^{\prime} \mathrm{W}^{-1} \mathrm{X}\right)^{-1} \mathrm{X}_{a}^{\prime} \mathrm{R}_{a}^{-1} \mathrm{Z}_{a} \mathrm{GZ} \mathrm{Z}_{o}^{\prime}+\mathrm{Z}^{\prime} \mathrm{R}^{-1} \mathrm{X} \\
& \left.\left.\cdot\left(\mathrm{X}^{\prime} \mathrm{W}^{-1} \mathrm{X}\right)^{-1} \mathrm{X}^{\prime} \mathrm{R}^{-1} \mathrm{Z}\left(\mathrm{Z}^{\prime} \mathrm{R}^{-1} \mathrm{Z}+\mathrm{G}^{-1}\right)^{-1} \mathrm{Z}_{a}^{\prime} \mathrm{R}_{a}^{-1} \mathrm{Z}_{a} \mathrm{GZ} \mathrm{Z}_{o}^{\prime}\right)-\mathrm{GZ}_{o}^{\prime}\right] \sigma^{2} \mathrm{I} \\
& =\left[( Z ^ { \prime } R ^ { - 1 } Z + G ^ { - 1 } ) ^ { - 1 } \left(Z^{\prime} R^{-1} Z_{G} Z_{o}^{\prime}-Z^{\prime} R^{-1} X\left(X^{\prime} W^{-1} X\right)^{-1} X^{\prime} R^{-1} Z G Z_{o}^{\prime}\right.\right. \\
& +\mathrm{Z}^{\prime} \mathrm{R}^{-1} \mathrm{X}\left(\mathrm{X}^{\prime} \mathrm{W}^{-1} \mathrm{X}\right)^{-1} \mathrm{X}^{\prime} \mathrm{R}^{-1} \mathrm{Z}\left(\mathrm{Z}^{\prime} \mathrm{R}^{-1} \mathrm{Z}+\mathrm{G}^{-1}\right)^{-1} \mathrm{Z}^{\prime} \mathrm{R}^{-1} \mathrm{ZGZ} \mathrm{Z}_{\circ}^{\prime}+\mathrm{Z}_{\circ}^{\prime} \\
& \left.\left.+\mathrm{Z}^{\prime} \mathrm{R}^{-1} \mathrm{X}\left(\mathrm{X}^{\prime} \mathrm{W}^{-1} \mathrm{X}\right)^{-1} \mathrm{X}^{\prime} \mathrm{R}^{-1} \mathrm{Z}\left(\mathrm{Z}^{\prime} \mathrm{R}^{-1} \mathrm{Z}+\mathrm{G}^{-1}\right)^{-1} \mathrm{Z}_{o}^{\prime}\right)-\mathrm{GZ}_{o}^{\prime}\right] \sigma^{2} \mathrm{l} \\
& =\left[( Z ^ { \prime } R ^ { - 1 } Z + G ^ { - 1 } ) ^ { - 1 } \left(Z^{\prime} R^{-1} Z G Z_{\circ}^{\prime}+Z_{\circ}^{\prime}-Z^{\prime} R^{-1} X\left(X^{\prime} W^{-1} X\right)^{-1} X^{\prime} R^{-1}\right.\right. \\
& \cdot Z G Z_{\circ}^{\prime}+Z^{\prime} R^{-1} X\left(X^{\prime} W^{-1} X\right)^{-1} X^{\prime} R^{-1} Z\left(Z^{\prime} R^{-1} Z+G^{-1}\right)^{-1}\left(Z^{\prime} R^{-1} Z+G^{-1}\right) \\
& \left.\left.\cdot \mathrm{GZ}_{\circ}^{\prime}\right)-\mathrm{GZ}_{\circ}^{\prime}\right] \sigma^{2} \mathrm{l} \\
& =\left[\left(\mathbf{Z}^{\prime} \mathrm{R}^{-1} \mathrm{Z}+\mathrm{G}^{-1}\right)^{-1}\left(\mathbf{Z}^{\prime} \mathrm{R}^{-1} \mathrm{ZG}+\mathbf{I}_{q}\right) \mathrm{Z}_{o}^{\prime}-\mathrm{GZ}_{o}^{\prime}\right] \sigma^{2} \mathrm{l}=0
\end{aligned}
$$




\subsection{SIMPLIFICAÇÃO DOS PREVISORES}

Na seção 2.2 deduzimos o BLUP dos efeitos fixos e aleatórios e das observações perdidas, dados pelas expressões (2.16), (2.18) e (2.22), respectivamente. Aqui estamos simplificando essas expressões, usando matrizes indicadoras dos dados presentes e ausentes.

Seja $\mathbf{E}$ a matriz indicadora dos dados ausentes e $\mathbf{F}$ a matriz indicadora dos dados presentes. E é uma matriz de dimensão $(d \times m)$, com cada coluna correspondendo a um dos dados perdidos. Cada coluna de $\mathbf{E}$ é composta por zero's e por um 1, localizado na linha cuja ordem é a mesma do dado perdido a que a coluna corresponde. A matriz $\mathrm{F}$ tem dimensão $(d \times(d-m))$ e é análoga a $\mathbf{E}$, só que indica os dados observados.

Para ilustrar a construção de $\mathbf{E}$ e $\mathbf{F}$, damos o seguinte exemplo.

Exemplo 2.3. Suponha que $d=5$ e que as duas últimas observações são perdidas. Assim,

$$
\mathrm{y}=\left(\begin{array}{l}
y_{1} \\
y_{2} \\
y_{3} \\
y_{4} \\
y_{5}
\end{array}\right) \quad \mathrm{y}_{0}=\left(\begin{array}{l}
y_{1} \\
y_{2} \\
y_{3}
\end{array}\right) \quad \mathrm{y}_{a}=\left(\begin{array}{l}
y_{4} \\
y_{5}
\end{array}\right)
$$

Nesse caso,

$$
\mathrm{E}=\left(\begin{array}{ll}
0 & 0 \\
0 & 0 \\
0 & 0 \\
1 & 0 \\
0 & 1
\end{array}\right) \quad \mathrm{F}=\left(\begin{array}{lll}
1 & 0 & 0 \\
0 & 1 & 0 \\
0 & 0 & 1 \\
0 & 0 & 0 \\
0 & 0 & 0
\end{array}\right)
$$

Usando essa notação, podemos observar que: 


$$
\begin{aligned}
\mathrm{y}_{o}=\mathrm{F}^{\prime} \mathrm{y} & \mathrm{y}_{a}=\mathrm{E}^{\prime} \mathrm{y} \\
\mathrm{X}_{o}=\mathrm{F}^{\prime} \mathrm{X} & \mathrm{X}_{a}=\mathrm{E}^{\prime} \mathrm{X} \\
\mathrm{Z}_{o}=\mathrm{F}^{\prime} \mathrm{Z} & \mathrm{Z}_{a}=\mathrm{E}^{\prime} \mathrm{Z} \\
\mathrm{R}_{o}^{-1}=\mathrm{F}^{\prime} \mathrm{R}^{-1} \mathrm{~F} & \mathrm{R}_{a}^{-1}=\mathrm{E}^{\prime} \mathrm{R}^{-1} \mathrm{E}
\end{aligned}
$$

e que

$$
\begin{array}{cr}
\mathrm{E}^{\prime} \mathrm{E}=\mathrm{I}_{m} & \mathrm{~F}^{\prime} \mathrm{F}=\mathrm{I}_{d-m} \\
\mathrm{E}^{\prime} \mathrm{F}=0 & \mathrm{EE}^{\prime}+\mathrm{FF}^{\prime}=\mathrm{I}_{d} \\
\mathrm{FF}^{\prime} \mathrm{R}^{-1} \mathrm{FF}^{\prime}+\mathrm{EE}^{\prime} \mathrm{R}^{-1} \mathrm{EE}^{\prime}=\mathrm{R}^{-1}
\end{array}
$$

Note que, se as observações perdidas fossem outras que não as últimas, ainda assim poderíamos utilizar as matrizes indicadoras $\mathbf{E}$ e F.

Exemplo 2.4. Considere o exemplo 2.3, só que agora os dados perdidos são o segundo e o quarto. Teríamos:

$$
\mathrm{y}=\left(\begin{array}{l}
y_{1} \\
y_{2} \\
y_{3} \\
y_{4} \\
y_{5}
\end{array}\right) \quad \mathrm{y}_{o}=\left(\begin{array}{l}
y_{1} \\
y_{3} \\
y_{5}
\end{array}\right) \quad \mathbf{y}_{a}=\left(\begin{array}{l}
y_{2} \\
y_{4}
\end{array}\right)
$$

com

$$
\mathrm{E}=\left(\begin{array}{ll}
0 & 0 \\
1 & 0 \\
0 & 0 \\
0 & 1 \\
0 & 0
\end{array}\right) \quad \mathrm{F}=\left(\begin{array}{lll}
1 & 0 & 0 \\
0 & 0 & 0 \\
0 & 1 & 0 \\
0 & 0 & 0 \\
0 & 0 & 1
\end{array}\right)
$$

e as igualdades dadas por (2.28) a (2.34) ainda seriam válidas. 
Substituindo as expressões (2.28) a (2.33) na expressão (2.22) temos,

$$
\begin{aligned}
& \hat{y}_{a}=\left\{I_{m}-E^{\prime} X\left(X^{\prime} W^{-1} X\right)^{-1}\left[X^{\prime} E-X^{\prime} R^{-1} Z A Z^{\prime} E\right] E^{\prime} R^{-1} E-E^{\prime} Z A\left[Z^{\prime} E-Z^{\prime} R^{-1} X\right.\right. \\
& \left.\left.\cdot\left(X^{\prime} W^{-1} X\right)^{-1}\left(X^{\prime} E-X^{\prime} R^{-1} Z A Z^{\prime} E\right)\right] E^{\prime} R^{-1} E\right\}^{-1}\left\{E^{\prime} X\left(X^{\prime} W^{-1} X\right)^{-1}\right. \\
& \cdot\left[\mathrm{X}^{\prime} \mathrm{F}-\mathrm{X}^{\prime} \mathrm{R}^{-1} \mathrm{ZAZ} \mathrm{Z}^{\prime} \mathrm{F}\right]+\mathrm{E}^{\prime} \mathrm{ZA}\left[\mathrm{Z}^{\prime} \mathrm{F}-\mathrm{Z}^{\prime} \mathrm{R}^{-1} \mathrm{X}\left(\mathrm{X}^{\prime} \mathrm{W}^{-1} \mathrm{X}\right)^{-1}\left(\mathrm{X}^{\prime} \mathrm{F}\right.\right. \\
& \left.\left.\left.-\mathrm{X}^{\prime} \mathrm{R}^{-1} \mathrm{ZAZ} \mathrm{F}\right)\right]\right\} \mathrm{F}^{\prime} \mathrm{R}^{-1} \mathrm{FF}^{\prime} \mathrm{y} \\
& =\left\{I_{m}-E^{\prime} X\left(X^{\prime} W^{-1} X\right)^{-1} X^{\prime} W^{-1} R E E^{\prime} R^{-1} E-E^{\prime} Z A\left[Z^{\prime} E-Z^{\prime} R^{-1} X\left(X^{\prime} W^{-1} X\right)^{-1}\right.\right. \\
& \left.\left.\cdot \mathrm{X}^{\prime} \mathrm{W}^{-1} \mathrm{RE}\right] \mathrm{E}^{\prime} \mathrm{R}^{-1} \mathrm{E}\right\}^{-1}\left\{\mathrm{E}^{\prime} \mathrm{X}\left(\mathrm{X}^{\prime} \mathrm{W}^{-1} \mathrm{X}\right)^{-1} \mathrm{X}^{\prime} \mathrm{W}^{-1} \mathrm{RFF} \mathrm{R}^{-1} \mathrm{~F}+\mathrm{E}^{\prime} \mathrm{ZA}\left[\mathrm{Z}^{\prime} \mathrm{FF}^{\prime} \mathrm{R}^{-1} \mathrm{~F}\right.\right. \\
& \left.\left.-Z^{\prime} R^{-1} X\left(X^{\prime} W^{-1} X\right)^{-1} X^{\prime} W^{-1} R F F^{\prime} R^{-1} F\right]\right\} F^{\prime} y
\end{aligned}
$$

Mas, usando o fato que $A=\left(Z^{\prime} R^{-1} Z+G^{-1}\right)^{-1}, W=Z G Z^{\prime}+R$ e a igualdade dada por (2.15), temos que,

$$
\begin{aligned}
& \hat{\mathrm{y}}_{a}=\left\{\mathrm{I}_{m}-\mathrm{E}^{\prime}\left(\mathrm{I}_{d}-\mathrm{ZAZ} \mathrm{Z}^{\prime} \mathrm{R}^{-1}\right) \mathrm{X}\left(\mathrm{X}^{\prime} \mathrm{W}^{-1} \mathrm{X}\right)^{-1} \mathrm{X}^{\prime} \mathrm{W}^{-1} \mathrm{REE} \mathrm{R}^{-1} \mathrm{E}-\mathrm{E}^{\prime} \mathrm{ZAZ} \mathrm{Z}^{\prime} \mathrm{EE}^{\prime} \mathrm{R}^{-1}\right. \\
& \cdot \mathrm{E}\}^{-1}\left\{\mathrm{E}^{\prime}\left(\mathrm{I}_{d}-\mathrm{ZAZ} \mathrm{Z}^{\prime} \mathrm{R}^{-1}\right) \mathrm{X}\left(\mathrm{X}^{\prime} \mathrm{W}^{-1} \mathrm{X}\right)^{-1} \mathrm{X}^{\prime} \mathrm{W}^{-1} \mathrm{RFF}^{\prime} \mathrm{R}^{-1} \mathrm{~F}+\mathrm{E}^{\prime} \mathrm{ZAZ} \mathrm{Z}^{\prime} \mathrm{F}\right\} \mathrm{F}^{\prime} \mathrm{y} \\
& =\left\{I_{m}-E^{\prime}\left[R W^{-1} X\left(X^{\prime} W^{-1} X\right)^{-1} X^{\prime} W^{-1} R+Z A Z^{\prime}\right] E E^{\prime} R^{-1} E\right\}^{-1} \\
& \cdot\left\{E^{\prime}\left[R W^{-1} X\left(X^{\prime} W^{-1} X\right)^{-1} X^{\prime} W^{-1} R+Z A Z^{\prime}\right] F F^{\prime} R^{-1} F\right\} F^{\prime} y \\
& =\left\{\mathrm{I}_{m}-\mathrm{E}^{\prime} \mathrm{R}\left[\mathrm{W}^{-1} \mathrm{X}\left(\mathrm{X}^{\prime} \mathrm{W}^{-1} \mathrm{X}\right)^{-1} \mathrm{X}^{\prime} \mathrm{W}^{-1}+\mathrm{R}^{-1}-\mathrm{W}^{-1}\right] \mathbf{R E} \mathrm{E}^{\prime} \mathbf{R}^{-1} \mathrm{E}\right\}^{-1} \\
& \cdot\left\{E^{\prime} R\left[W^{-1} X\left(X^{\prime} W^{-1} X\right)^{-1} X^{\prime} W^{-1}+R^{-1}-W^{-1}\right] R F F^{\prime} R^{-1} F\right\} F^{\prime} y
\end{aligned}
$$

Assim, $\hat{\mathrm{y}}_{a}$ é dado por

$$
\hat{\mathrm{y}}_{a}=\left(\mathrm{I}_{m}-\mathrm{E}^{\prime} \mathrm{QEE} \mathrm{R}^{-1} \mathrm{E}\right)^{-1} \mathrm{E}^{\prime} \mathrm{QFF} \mathrm{R}^{-1} \mathrm{FF}^{\prime} \mathrm{y}
$$


onde

$$
\mathrm{Q}=\mathrm{R}\left[\mathrm{W}^{-1} \mathrm{X}\left(\mathrm{X}^{\prime} \mathrm{W}^{-1} \mathrm{X}\right)^{-1} \mathrm{X}^{\prime} \mathrm{W}^{-1}+\mathrm{R}^{-1}-\mathrm{W}^{-1}\right] \mathrm{R} .
$$

É interessante salientar que o BLUP das observações ausentes, dado por (2.35) e (2.36), não depende de $\mathrm{Z}$, a matriz de planejamento dos efeitos aleatórios.

Para simplificar $\hat{\alpha}$ e $\hat{b}$, dados por (2.16) e (2.18) respectivamente, basta substituir nessas expressões, as igualdades dadas por (2.28) a (2.33) e então

$$
\hat{\alpha}=\left(\mathrm{X}^{\prime} \mathrm{W}^{-1} \mathrm{X}\right)^{-1} \mathrm{X}^{\prime} \mathrm{W}^{-1} \mathrm{R}\left(\mathrm{FF}^{\prime} \mathrm{R}^{-1} \mathrm{FF}^{\prime} \mathrm{y}+\mathrm{EE}^{\prime} \mathrm{R}^{-1} \mathrm{E} \hat{\mathrm{y}}_{a}\right)
$$

$\mathrm{e}$

$$
\hat{\mathrm{b}}=\mathrm{AZ} \mathrm{Z}^{\prime}\left[\mathrm{I}_{d}-\mathrm{R}^{-1} \mathrm{X}\left(\mathrm{X}^{\prime} \mathrm{W}^{-1} \mathrm{X}\right)^{-1} \mathrm{X}^{\prime} \mathrm{W}^{-1} \mathrm{R}\right]\left[\mathrm{FF}^{\prime} \mathrm{R}^{-1} \mathrm{FF}^{\prime} \mathrm{y}+\mathrm{EE}^{\prime} \mathrm{R}^{-1} \mathrm{E} \hat{\mathrm{y}}_{a}\right]
$$

Se os erros são independentes, ou seja, se $\mathrm{R}=\mathrm{I}_{d}$, os BLUP's de $\mathrm{y}_{a}, \alpha$ e b serão:

$$
\hat{\mathrm{y}}_{a}=\left(\mathrm{I}_{m}-\mathrm{E}^{\prime} \mathrm{QE}\right)^{-1} \mathrm{E}^{\prime} \mathrm{QFF} \mathrm{F}^{\prime} \mathrm{y}
$$

onde

$$
\begin{gathered}
\mathrm{Q}=\mathrm{W}^{-1} \mathrm{X}\left(\mathrm{X}^{\prime} \mathrm{W}^{-1} \mathrm{X}\right)^{-1} \mathrm{X}^{\prime} \mathrm{W}^{-1}+\mathrm{I}_{d}-\mathrm{W}^{-1}, \\
\hat{\alpha}=\left(\mathrm{X}^{\prime} \mathrm{W}^{-1} \mathrm{X}\right)^{-1} \mathrm{X}^{\prime} \mathrm{W}^{-1}\left(\mathrm{FF}^{\prime} \mathrm{y}+\mathrm{E} \hat{\mathrm{Y}}_{a}\right)
\end{gathered}
$$

e 


$$
\hat{\mathrm{b}}=\mathrm{AZ} \mathrm{Z}^{\prime}\left[\mathrm{I}_{d}-\mathrm{X}\left(\mathrm{X}^{\prime} \mathrm{W}^{-1} \mathrm{X}\right)^{-1} \mathrm{X}^{\prime} \mathrm{W}^{-1}\right]\left[\mathrm{FF}^{\prime} \mathrm{y}+\mathrm{E} \hat{y}_{a}\right]
$$

\subsection{ERRO QUADRÁTRICO MÉDIO DOS PREVISORES}

Nesta seção calculamos o erro quadrático médio dos previsores obtidos anteriormente. i. EQM de $\hat{\mathrm{y}}_{a}$

$$
\begin{gathered}
\operatorname{EQM}\left(\hat{\mathrm{y}}_{a}\right)=\mathbb{E}\left[\left(\hat{\mathrm{y}}_{a}-\mathrm{y}_{a}\right)\left(\hat{\mathrm{y}}_{a}-\mathrm{y}_{a}\right)^{\prime}\right] \\
=\mathbb{E}\left[( \mathrm { I } _ { m } - \mathrm { E } ^ { \prime } \mathrm { QEE } \mathrm { E } ^ { - 1 } \mathrm { E } ) ^ { - 1 } \mathrm { E } ^ { \prime } \mathrm { Q } \mathrm { FF } ^ { \prime } \mathrm { R } ^ { - 1 } \mathrm { FF } ^ { \prime } \mathrm { yy } ^ { \prime } \mathrm { FF } ^ { \prime } \mathrm { R } ^ { - 1 } \mathrm { FF } ^ { \prime } \mathrm { QE } \left(\mathrm{I}_{m}\right.\right. \\
\left.\left.-\mathrm{E}^{\prime} \mathrm{R}^{-1} \mathrm{EE}^{\prime} \mathrm{QE}\right)^{-1}\right]-\mathbb{E}\left[\left(\mathrm{I}_{m}-\mathrm{E}^{\prime} \mathrm{QEE} \mathrm{E}^{\prime} \mathrm{R}^{-1} \mathrm{E}\right)^{-1} \mathrm{E}^{\prime} \mathrm{QFF} \mathrm{F}^{\prime} \mathrm{R}^{-1} \mathrm{FF}^{\prime} \mathrm{y}_{a}^{\prime}\right] \\
-\mathbb{E}\left[\mathrm{y}_{a} \mathrm{y}^{\prime} \mathrm{FF}^{\prime} \mathrm{R}^{-1} \mathrm{FF}^{\prime} \mathrm{QE}\left(\mathrm{I}_{m}-\mathrm{E}^{\prime} \mathrm{R}^{-1} \mathrm{EE}^{\prime} \mathrm{QE}\right)^{-1}\right]+\mathbb{E}\left(\mathrm{y}_{a} \mathrm{y}_{a}^{\prime}\right) .
\end{gathered}
$$

Isso leva a

$$
\begin{aligned}
E Q M\left(\hat{\mathrm{y}}_{a}\right) & =\left[\left(\mathrm{I}_{m}-\mathrm{E}^{\prime} \mathrm{QEE} \mathrm{E}^{\prime} \mathrm{R}^{-1} \mathrm{E}\right)^{-1} \mathrm{E}^{\prime} \mathrm{QFF} \mathrm{F}^{\prime} \mathrm{R}^{-1} \mathrm{FF}^{\prime}-\mathrm{E}^{\prime}\right] \mathrm{W} \\
& \cdot\left[\mathrm{FF}^{\prime} \mathrm{R}^{-1} \mathrm{FF}^{\prime} \mathrm{QE}\left(\mathrm{I}_{m}-\mathrm{E}^{\prime} \mathrm{R}^{-1} \mathrm{EE}^{\prime} \mathrm{QE}\right)^{-1}-\mathrm{E}\right] \sigma^{2}
\end{aligned}
$$

Se os erros são independentes,

$$
\begin{aligned}
\operatorname{EQM}\left(\hat{\mathrm{y}}_{a}\right) & =\left[\left(\mathrm{I}_{m}-\mathrm{E}^{\prime} \mathrm{QE}\right)^{-1} \mathrm{E}^{\prime} \mathrm{QFF} F^{\prime}-\mathrm{E}^{\prime}\right] \mathrm{W} \\
& \cdot\left[\mathrm{FF}^{\prime} \mathrm{QE}\left(\mathrm{I}_{m}-\mathrm{E}^{\prime} \mathrm{QE}\right)^{-1}-\mathrm{E}\right] \sigma^{2}
\end{aligned}
$$

ii. EQM de $\hat{\alpha}$

Seja 
$\mathbf{B}=\left(\mathrm{X}^{\prime} \mathrm{W}^{-1} \mathrm{X}\right)^{-1} \mathrm{X}^{\prime} \mathrm{W}^{-1} \mathrm{R}\left[\mathrm{I}_{d}+\mathrm{EE}^{\prime} \mathrm{R}^{-1} \mathrm{E}\left(\mathrm{I}_{m}-\mathrm{E}^{\prime} \mathrm{QEE} \mathrm{E}^{-1} \mathrm{E}\right)^{-1} \mathrm{E}^{\prime} \mathrm{Q}\right] \mathrm{FF}^{\prime} \mathrm{R}^{-1} \mathrm{FF}^{\prime}$

O erro quadrático médio de $\hat{\alpha}$ é dado por:

$$
E Q M(\hat{\alpha})=\mathrm{BWB}^{\prime} \sigma^{2}
$$

Se os erros são independentes,

$$
\mathrm{B}=\left(\mathrm{X}^{\prime} \mathrm{W}^{-1} \mathrm{X}\right)^{-1} \mathrm{X}^{\prime} \mathrm{W}^{-1}\left[\mathrm{I}_{d}+\mathrm{E}\left(\mathrm{I}_{m}-\mathrm{E}^{\prime} \mathrm{QE}\right)^{-1} \mathrm{E}^{\prime} \mathrm{Q}\right] \mathrm{FF}^{\prime} .
$$

iii. EQM de $\hat{b}$

Seja

$$
\begin{aligned}
\mathrm{C}= & \mathrm{AZ}\left[\mathrm{Z}_{d}-\mathrm{R}^{-1} \mathrm{X}\left(\mathrm{X}^{\prime} \mathrm{W}^{-1} \mathrm{X}\right)^{-1} \mathrm{X}^{\prime} \mathrm{W}^{-1} \mathrm{R}\right]\left[\mathrm{I}_{d}+\mathrm{EE}^{\prime} \mathrm{R}^{-1} \mathrm{E}\left(\mathrm{I}_{m}-\mathrm{E}^{\prime} \mathrm{Q} \mathrm{EE}^{\prime} \mathrm{R}^{-1} \mathrm{E}\right)^{-1} \mathrm{E}^{\prime} \mathrm{Q}\right] \\
& \cdot \mathrm{FF} \mathrm{F}^{\prime} \mathbf{R}^{-1} \mathrm{FF}^{\prime} .
\end{aligned}
$$

O erro quadrático médio de $\hat{b}$ é dado por:

$$
E Q M(\hat{\mathrm{b}})=\left(\mathrm{CWC} \mathrm{C}^{\prime}-\mathrm{CZG}-\mathrm{GZ}^{\prime} \mathrm{C}^{\prime}+\mathrm{G}\right) \sigma^{2}
$$

Se os erros são independentes,

$$
\mathrm{C}=\mathrm{AZ}\left[\mathrm{I}_{d}-\mathrm{X}\left(\mathrm{X}^{\prime} \mathrm{W}^{-1} \mathrm{X}\right)^{-1} \mathrm{X}^{\prime} \mathrm{W}^{-1}\right]\left[\mathrm{I}_{d}+\mathrm{E}\left(\mathrm{I}_{m}-\mathrm{E}^{\prime} \mathrm{QE}\right)^{-1} \mathrm{E}^{\prime} \mathrm{Q}\right] \mathrm{FF}^{\prime}
$$




\section{CAPÍTULO 3}

\section{CASO UNIVARIADO}

\subsection{INTRODUÇÃO}

Neste capitulo estamos aplicando a técnica desenvolvida no capítulo anterior ao nosso problema específico, isto é, estamos fazendo a imputação das observações perdidas pelo previsor linear não-viesado de erro quadrático médio mínimo (BLUP).

O problema: para $n$ indivíduos, estamos medindo uma variável em $T$ ocasiões diferentes, isto é, estamos considerando um painel. Os $n$ indivíduos foram sorteados, sem reposição, de uma população finita de tamanho $N$ e algumas das observações são perdidas. O nosso problema é obter o BLUP para essas observações.

Adotamos o modelo usual para medidas repetidas, no qual o tempo é um fator fixo e 
o indivíduo um fator aleatório.

Nos livros e trabalhos tradicionais é comum supor que os efeitos aleatórios são extraídos de uma população infinita. Para populações finitas, os modelos foram estudados por alguns autores, entre os quais podemos citar Bennett e Franklin (1954), Cornfield e Tukey (1956), Tukey (1956a, 1957a e 1957b), Gaylor e Hartwell (1969), Searle e Fawcett (1970) e Bussab e Barroso (1992).

Searle e Fawcett (1970) desenvolveram uma regra para converter esperanças no modelo com população infinita para esperanças no modelo com população finita e apresentaram estimadores da componente de variância entre classes, para os dois modelos, no caso univariado. Bennett e Franklin (1954) trabalharam com um modelo misto, no sentido que o fator aleatório provinha de uma população finita, enquanto que a componente de erro mantinha a suposição de normalidade. Bussab e Barroso (1992) estenderam a regra desenvolvida por Searle e Fawcett (1970) para o caso multivariado, considerando o modelo misto com dois fatores, um fixo e um aleatório e considerando também, como Bennett e Franklin (1954) uma mistura de população finita com população infinita. É interessante salientar que esse modelo é misto sob dois pontos de vista: um, porque mistura efeito fixo com aleatório; outro, porque mistura população finita com infinita.

Adotamos o modelo com dois fatores sem interação pois, conforme Searle (1971), quando há somente uma observação por casela, o modelo com interação torna-se indistinguível do modelo sem interação. Assim, consideramos o tempo como um fator fixo e o indivíduo como um fator aleatório, ou seja, os dados seguem o seguinte modelo linear:

$$
y_{t i}=\mu+\alpha_{t}+b_{i}+e_{t i} \quad \begin{aligned}
t & =1,2, \ldots, T \\
i & =1,2, \ldots, n
\end{aligned}
$$


onde

$y_{t i}:$ observação do indivíduo $i$ no instante $t$;

$\mu$ : média geral;

$\alpha_{t}$ : fator fixo medindo o efeito do tempo;

$b_{i}$ : fator aleatório medindo o efeito do indivíduo;

$e_{t i}$ : erro associado ao indivíduo $i$ no instante $t$.

Podemos também escrever o modelo como:

$$
y_{t i}=\mu_{t}+b_{i}+e_{t i}
$$

onde $\mu_{t}$ é a média no $t$-ésimo instante.

As suposições adotadas são:

$$
\begin{aligned}
& e_{t i} \sim N\left(0, \sigma^{2}\right) \\
& e_{t i}^{\prime} \text { ' independentes; } \\
& e_{t i}^{\prime} \text { s e } b_{i} \text { 's independentes. }
\end{aligned}
$$

Definimos

$$
\sum_{t=1}^{T} \alpha_{t}=0, \quad \sum_{i=1}^{N} b_{i}=0 \quad \text { e } \quad S^{2}=\frac{1}{N-1} \sum_{i=1}^{N} b_{i}^{2} .
$$

Seja y o vetor de todas as observações, dado por:

$$
\mathrm{y}^{\prime}=\left(y_{11}, y_{21}, \ldots, y_{T 1}, y_{12}, y_{22}, \ldots, y_{T 2}, \ldots, y_{1 n}, y_{2 n}, \ldots, y_{T n}\right)
$$

Com base nas suposições acima e observando que:

i)

$$
0=\left(\sum_{i=1}^{N} b_{i}\right)\left(\sum_{i^{\prime}=1}^{N} b_{i^{\prime}}\right)=\sum_{i=1}^{N} b_{i}^{2}+\sum_{i=1}^{N} \sum_{\substack{i^{\prime}=1 \\ i^{\prime} \neq i}}^{N} b_{i} b_{i^{\prime}}=(N-1) S^{2}+\sum_{i=1}^{N} \sum_{\substack{i^{\prime}=1 \\ i^{\prime} \neq i}}^{N} b_{i} b_{i^{\prime}}
$$




$$
\Longrightarrow \sum_{i=1}^{N} \sum_{\substack{i^{\prime}=1 \\ i^{\prime} \neq i}}^{N} b_{i} b_{i^{\prime}}=-(N-1) S^{2}
$$

ii) Para quaisquer $j, j^{\prime}, j, j^{\prime}=1,2, \ldots, N$

$$
\begin{aligned}
& \mathbb{E}\left(b_{j}\right)=\frac{1}{N} \sum_{i=1}^{N} b_{i}=0 \\
& \operatorname{Var}\left(b_{j}\right)=\mathbb{E}\left(b_{j}^{2}\right)=\frac{1}{N} \sum_{i=1}^{N} b_{i}^{2}=\left(1-N^{-1}\right) S^{2} \\
& \operatorname{Cov}\left(b_{j}, b_{j^{\prime}}\right)=\mathbb{E}\left(b_{j^{\prime}} b_{j^{\prime}}\right)=\frac{1}{N(N-1)} \sum_{\substack{i=1 \\
i^{\prime}=1 \\
i^{\prime} \neq i}}^{N} b_{i} b_{i^{\prime}}=-\frac{S^{2}}{N},
\end{aligned}
$$

podemos calcular as covariâncias entre os elementos do vetor y. Assim:

dentro do indivíduo

no mesmo tempo

$$
\operatorname{Var}\left(y_{t i}\right)=\operatorname{Var}\left(b_{i}\right)+\operatorname{Var}\left(e_{t i}\right)=\left(1-N^{-1}\right) S^{2}+\sigma^{2}
$$

entre tempos

$$
\operatorname{Cov}\left(y_{t i}, y_{t^{\prime} i}\right)=\operatorname{Cov}\left(\mu_{t}+b_{i}+e_{t i}, \mu_{t^{\prime}}+b_{i}+e_{t^{\prime} i}\right)=\left(1-N^{-1}\right) S^{2}
$$

entre indivíduos

$$
\operatorname{Cov}\left(y_{t i}, y_{t^{\prime} i^{\prime}}\right)=\operatorname{Cov}\left(\mu_{t}+b_{i}+e_{t i}, \mu_{t^{\prime}}+b_{i^{\prime}}+e_{t^{\prime} i^{\prime}}\right)=-\frac{S^{2}}{N} .
$$

É importante salientar que este modelo é limitado já que impõe a mesma estrutura de covariâncias entre tempos diferentes, quer eles sejam próximos ou não.

Assim,

$$
\mathrm{V}=\operatorname{Var}(\mathbf{y})=\mathbf{W} \sigma^{2}=\left[\left(\mathbf{I}_{n} \otimes \mathbf{I}_{T}\right)+K\left(\mathbf{I}_{n} \otimes \mathbf{J}_{T}\right)-\frac{K}{N}\left(\mathbf{J}_{n} \otimes \mathbf{J}_{T}\right)\right] \sigma^{2}
$$


onde

$\mathbf{I}_{n}$ : matriz identidade de dimensão $n$;

$\mathfrak{J}_{T}$ : matriz quadrada de 1's de dimensão $T$;

Q: produto de Kronecker (veja definição e propriedades no apêndice A.1).

$K=\frac{S^{2}}{\sigma^{2}}:$ razão entre as componentes da variância,

ou seja,

$$
\mathrm{W}=\left(\begin{array}{cccc}
\mathrm{C}+\mathrm{B} & \mathrm{C} & \cdots & \mathrm{C} \\
\mathrm{C} & \mathrm{C}+\mathrm{B} & \cdots & \mathrm{C} \\
\vdots & \vdots & \vdots & \vdots \\
\mathrm{C} & \mathrm{C} & \cdots & \mathrm{C}+\mathrm{B}
\end{array}\right)
$$

onde

$$
\mathrm{C}=-\frac{K}{N} \mathbf{J}_{T} \quad \text { e } \quad \mathrm{B}=\mathrm{I}_{T}+K \mathrm{~J}_{T}
$$

isto é

$$
\mathrm{C}=-\frac{K}{N}\left(\begin{array}{cccc}
1 & 1 & \cdots & 1 \\
1 & 1 & \cdots & 1 \\
\vdots & \vdots & \vdots & \vdots \\
1 & 1 & \cdots & 1
\end{array}\right) \quad \text { e } \mathrm{B}=\left(\begin{array}{cccc}
1+K & K & \cdots & K \\
K & 1+K & \cdots & K \\
\vdots & \vdots & \vdots & \vdots \\
K & K & \cdots & 1+K
\end{array}\right)
$$

A matriz $\mathbf{C}+\mathbf{B}$ reflete as covariâncias "dentro do indivíduo", enquanto que a matriz C reflete as covariâncias "entre indivíduos", devidas ao sorteio sem reposição.

É interessante notar que, no modelo usualmente utilizado para populações infinitas, onde se faz a suposição de que os $b_{i}$ 's são independentes, a matriz de covariâncias de y é dada por:

$$
\mathbf{V}_{\infty}=\mathbf{W}_{\infty} \sigma^{2}=\left[\left(\mathbf{I}_{n} \otimes \mathbf{I}_{T}\right)+K\left(\mathbf{I}_{n} \otimes \mathbf{J}_{T}\right)\right] \sigma^{2}
$$

ou seja, 


$$
\mathrm{W}_{\infty}=\left(\begin{array}{cccc}
\mathrm{B} & 0 & \cdots & 0 \\
0 & \mathrm{~B} & \cdots & 0 \\
\vdots & \vdots & \vdots & \vdots \\
0 & 0 & \cdots & \mathrm{B}
\end{array}\right) .
$$

Enquanto $\mathrm{V}_{\infty}$ é bloco-diagonal, em geral $\mathrm{V}$ é uma matriz "cheia", com todos os elementos diferentes de zero. A comparação entre essas matrizes leva à mesma idéia que aparece em amostragem, quando da multiplicação pelo fator de correção para populações finitas no cálculo das variâncias dos estimadores (ver Cochran(1977) ou Kish (1965)).

No modelo adotado, as matrizes $\mathrm{X}$ e $\mathrm{Z}$ são dadas por:

$$
\mathrm{X}=\left(\begin{array}{cccc}
1 & 0 & \cdots & 0 \\
0 & 1 & \cdots & 0 \\
\vdots & \vdots & \vdots & \vdots \\
0 & 0 & \cdots & 1 \\
1 & 0 & \cdots & 0 \\
0 & 1 & \cdots & 0 \\
\vdots & \vdots & \vdots & \vdots \\
0 & 0 & \cdots & 1 \\
\vdots & \vdots & \vdots & \vdots \\
1 & 0 & \cdots & 0 \\
0 & 1 & \cdots & 0 \\
\vdots & \vdots & \vdots & \vdots \\
0 & 0 & \cdots & 1
\end{array}\right) \quad \mathrm{Z}=\left(\begin{array}{cccc}
1 & 0 & \cdots & 0 \\
1 & 0 & \cdots & 0 \\
\vdots & \vdots & \vdots & \vdots \\
1 & 0 & \cdots & 0 \\
0 & 1 & \cdots & 0 \\
0 & 1 & \cdots & 0 \\
\vdots & \vdots & \vdots & \vdots \\
0 & 1 & \cdots & 0 \\
\vdots & \vdots & \vdots & \vdots \\
0 & 0 & \cdots & 1 \\
0 & 0 & \cdots & 1 \\
\vdots & \vdots & \vdots & \vdots \\
0 & 0 & \cdots & 1
\end{array}\right)
$$

isto é,

$$
\mathrm{X}=\mathrm{I}_{n} \otimes \mathrm{I}_{T} \quad \text { e } \quad \mathrm{Z}=\mathrm{I}_{n} \otimes \mathrm{I}_{T},
$$

onde $1_{n}$ indica o vetor coluna de 1 's de dimensão $n$.

A dedução do BLUP das observações ausentes é a aplicação direta da expressão (2.39), onde 


$$
\begin{aligned}
& \mathrm{W}^{-1}=\left(\mathrm{I}_{n} \otimes \mathbf{I}_{T}\right)-a\left(\mathrm{I}_{n} \otimes \mathbf{J}_{T}\right)+b\left(\mathbf{J}_{n} \otimes \mathbf{J}_{T}\right), \\
& \left(\mathrm{X}^{\prime} \mathbf{W}^{-1} \mathrm{X}\right)^{-1}=\frac{1}{n}\left[\mathbf{I}_{T}-\frac{(n b-a)}{(T(n b-a)+1)} \mathbf{J}_{T}\right], \\
& \mathbf{Q}=\frac{1}{n}\left(\mathbf{J}_{n} \otimes \mathbf{I}_{T}\right)+a\left(\mathrm{I}_{n} \otimes \mathbf{J}_{T}\right)-\frac{a}{n}\left(\mathbf{J}_{n} \otimes \mathbf{J}_{T}\right),
\end{aligned}
$$

com $a=\frac{K}{(T K+1)} \quad, \quad b=\frac{K}{N(T K+1)(T K(1-f)+1)} \quad$ e $\quad f=\frac{n}{N}$ (fração amostral) .

\subsection{CASO 1: UMA OBSERVAÇÃO PERDIDA NO ÚLTIMO INSTANTE}

O primeiro caso que estudamos é aquele em que uma única observação é ausente. Sem perda de generalidade, supomos que essa observação corresponde ao $n$-ésimo indivíduo no instante $T$. Assim, queremos prever $y_{T n}$, no seguinte esquema de dados:

\begin{tabular}{c|cccc|c} 
& \multicolumn{5}{|c|}{ tempo } \\
indiv. & 1 & 2 & $\cdots$ & $T-1$ & $T$ \\
\hline 1 & $y_{11}$ & $y_{21}$ & $\cdots$ & $y_{(T-1), 1}$ & $y_{T 1}$ \\
2 & $y_{12}$ & $y_{22}$ & $\cdots$ & $y_{(T-1), 2}$ & $y_{T 2}$ \\
$\vdots$ & $\vdots$ & $\vdots$ & $\cdots$ & $\vdots$ & $\vdots$ \\
$n-1$ & $y_{1,(n-1)}$ & $y_{2,(n-1)}$ & $\cdots$ & $y_{(T-1),(n-1)}$ & $y_{T,(n-1)}$ \\
\hline$n$ & $y_{1 n}$ & $y_{2 n}$ & $\cdots$ & $y_{(T-1), n}$ & $? ?$
\end{tabular}

Para obter o BLUP, basta identificar as matrizes $\mathrm{F}$ e $\mathrm{E}$, indicadoras dos dados presentes e ausentes, e aplicar a expressão (2.39), com $\mathrm{W}^{-1}$ e $\left(\mathrm{X}^{\prime} \mathrm{W}^{-1} \mathrm{X}\right)^{-1}$ dados por (3.6) e (3.7), respectivamente. 
Neste caso, as matrizes $\mathbf{F}$ e $\mathbf{E}$ são obtidas da partição da matriz identidade de dimensão $n T, \mathbf{F}$ correspondendo às $(n T-1)$ primeiras colunas de $\mathbf{I}_{n T}$ e $\mathbf{E}$ correspondendo à última coluna. Assim,

e

$$
\mathrm{E}=\left(\begin{array}{c}
0 \\
0 \\
\vdots \\
0 \\
1
\end{array}\right) \quad \mathbf{F}=\left(\begin{array}{cccc}
1 & 0 & \cdots & 0 \\
0 & 1 & \cdots & 0 \\
\vdots & \vdots & \vdots & \vdots \\
0 & 0 & \cdots & 1 \\
0 & 0 & \cdots & 0
\end{array}\right)
$$

$$
\hat{y}_{T n}=\bar{y}_{T}+\left(\frac{K(T-1)}{K(T-1)+1}\right)\left(\bar{y}_{. n}-\bar{y}_{c}\right)
$$

onde

$K=\frac{S^{2}}{\sigma^{2}}:$ razão entre as componentes da variância;

$\bar{y}_{c}=\frac{1}{(n-1)(T-1)} \sum_{t=1}^{T-1} \sum_{i=1}^{n-1} y_{t i}:$ média da parte completa dos dados, ou seja, média das observações dos $n-1$ indivíduos completos nos $T-1$ primeiros instantes;

$\bar{y}_{T .}=\frac{1}{n-1} \sum_{i=1}^{n-1} y_{T i}:$ média das observações no instante $T$;

$\bar{y}_{. n}=\frac{1}{T-1} \sum_{t=1}^{T-1} y_{t n}:$ média das observações do indivíduo $n$.

A demonstração destes resultados está feita em detalhes no apêndice B.1.

É interessante verificar que o previsor, dado por (3.9) é a média no instante $T$ mais uma correção que envolve a diferença entre a média do indivíduo com o dado ausente e a média dos demais. Se não há efeito de indivíduos, então $S^{2}=0, K=0$ e essa correção desaparece. Nesse caso, o BLUP é a média no último instante, $\bar{y}_{T}$.

Se a variância do efeito de indivíduos é muito maior do que a variância dos erros, isto é, $K \longrightarrow \infty$, entâo o previsor tende a $\bar{y}_{T}+\bar{y}_{. n}-\bar{y}_{c}$. Nesse caso, se não há efeito do tempo, $\bar{y}_{T}$. se aproxima de $\bar{y}_{c}$ e o BLUP é a média do $n$-ésimo indivíduo $\bar{y}_{. n}$. 
Se os dados são observados em um só instante, $T=1$, a correção não existe e o BLUP se reduz à média nesse instante. Se um único indivíduo é observado em $T$ instantes, $n=1$ e o BLUP não existe.

Outra observação importante é que para se obter o BLUP não é necessário conhecer cada uma das componentes da variância, basta conhecer a razão entre elas. Essa situação aparece comumente na prática, no estudo da variação de processos industriais.

Damos abaixo um exemplo numérico, como ilustração do comportamento do previsor dado por (3.9).

Exemplo 3.1. Voltando ao exemplo 1.1, o gráfico abaixo mostra os dados de total de salários (em milhões de cruzeiros reais) de cinco indústrias do setor de Papel e Papelão do Estado de São Paulo, do período de janeiro a abril de 1994.

\section{Total de Salários}

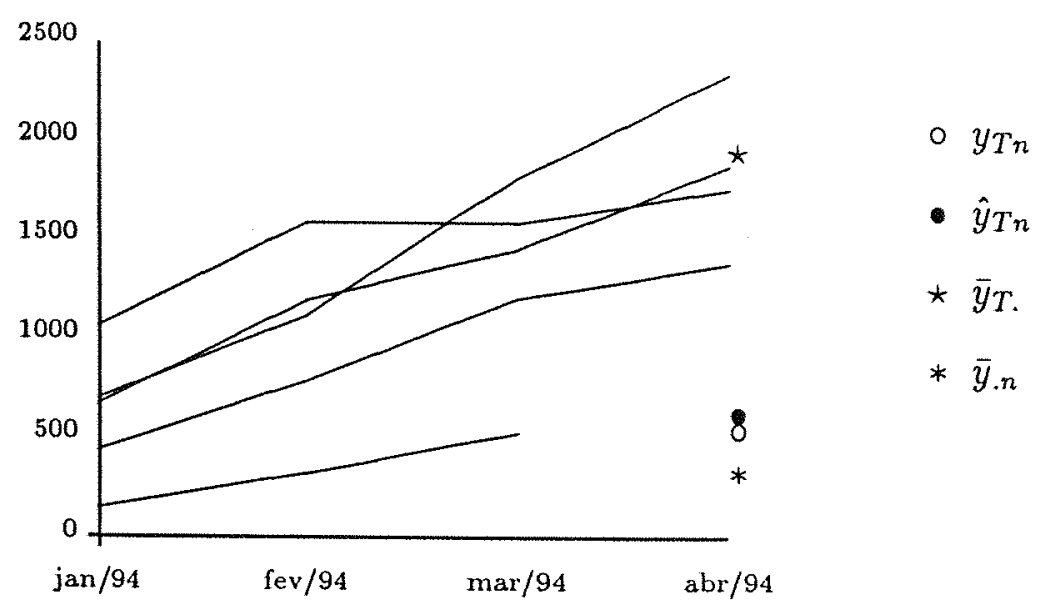


Esse gráfico ilustra a idéia de como atua o previsor aqui sugerido, ao corrigir a média no instante $T$.

O erro quadrático médio de $\hat{y}_{T n}$, cuja dedução encontra-se no apêndice B.6, é dado por:

$$
E Q M\left(\hat{y}_{T_{n}}\right)=\frac{n}{n-1}\left(\frac{K T+1}{K(T-1)+1}\right) \sigma^{2} .
$$

Se considerarmos a média no instante $T$, o erro quadrático médio do previsor é:

$$
\operatorname{EQM}\left(\bar{y}_{T .}\right)=\frac{n}{n-1}(K+1) \sigma^{2} .
$$

Comparando-se as duas expressões, temos que

$$
\frac{K T+1}{K(T-1)+1}=1+\frac{K}{K(T-1)+1} \leq K+1
$$

e portanto

$$
\operatorname{EQM}\left(\hat{y}_{T_{n}}\right) \leq \operatorname{EQM}\left(\bar{y}_{T .}\right)
$$

Quanto maior for o valor de $K$, maior será o ganho em se usar o BLUP no lugar da média no último instante.

Com relação à média do $n$-ésimo indivíduo, esse previsor é viesado e portanto não estamos comparando os correspondentes erros quadráticos médios. Se não existe efeito do tempo, $\bar{y}_{. n}$ é não-viesado, com erro quadrático médio igual a

$$
\operatorname{EQM}\left(\bar{y}_{. n}\right)=\frac{T}{T-1} \sigma^{2}
$$




\subsubsection{CONEXÃO COM O CASO DE POPULAÇÕES INFINITAS}

Para fazer a conexão com o caso de populações infinitas, recalculamos o BLUP de $y_{T n}$, utilizando os multiplicadores de Lagrange. Escrevendo o modelo como $y_{t i}=\mu_{t}+b_{i}+e_{t i}$, o problema consiste em obter uma combinação linear dos elementos do vetor $y, c^{\prime} y$, que minimize $\mathbb{E}\left[\mathbf{c}^{\prime} \mathbf{y}-\left(\mu_{T}+b_{n}+e_{T n}\right)\right]^{2}$, sujeita às seguintes condições:

$$
\begin{gathered}
c_{T n}=0 \\
\mathbb{E}\left(\mathrm{c}^{\prime} \mathrm{y}\right)=\mu_{T} .
\end{gathered}
$$

Essas condições implicam em:

$$
\left\{\begin{array}{l}
\text { para } t=T, \quad \sum_{i=1}^{n-1} c_{T i}=1 \text { e } \sum_{i=1}^{n} c_{T i}=1, \\
\text { para qualquer } t \neq T, \quad \sum_{i=1}^{n} c_{t i}=0 .
\end{array}\right.
$$

pois

$$
\mathbb{E}\left(\mathrm{c}^{\prime} \mathbf{y}\right)=\mathbb{E}\left(\sum_{i=1}^{n} \sum_{t=1}^{T} c_{t i} y_{t i}\right)=\sum_{t=1}^{T} \mu_{t} \sum_{i=1}^{n} c_{t i} .
$$

Assim,

$$
\begin{gathered}
\mathbb{E}\left[\mathbf{c}^{\prime} \mathbf{y}-\left(\mu_{T}+b_{n}+e_{T n}\right)\right]^{2} \\
=\mathbb{E}\left[\left(\sum_{i=1}^{n} \sum_{t=1}^{T} c_{t i}\left(\mu_{t}+b_{i}+e_{t i}\right)-\left(\mu_{T}+b_{n}+e_{T n}\right)\right]^{2}\right. \\
=\mathbb{E}\left[\left(\left(\sum_{i=1}^{n} \sum_{t=1}^{T} c_{t i} b_{i}-b_{n}\right)+\left(\sum_{i=1}^{n} \sum_{t=1}^{T} c_{t i} e_{t i}-e_{T n}\right)\right)^{2}\right]
\end{gathered}
$$




$$
\begin{aligned}
& =\mathbb{E}\left[\left(\sum_{i=1}^{n} \sum_{t=1}^{T} c_{t i} b_{i}-b_{n}\right)^{2}+\left(\sum_{i=1}^{n} \sum_{t=1}^{T} c_{t i} e_{t i}-e_{T n}\right)^{2}\right] \\
& =\mathbb{E}\left[\left(\sum_{i=1}^{n} c_{. i} b_{i}-b_{n}\right)^{2}+\left(\sum_{i=1}^{n} \sum_{t=1}^{T} c_{t i} e_{t i}-e_{T n}\right)^{2}\right]
\end{aligned}
$$

onde $c_{. i}=\sum_{t=1}^{T} c_{t i}$

Seja $\quad \tilde{c}_{. i}=c_{. i} \quad$ para $i=1,2, \ldots, n-1$

$$
\begin{aligned}
& \tilde{c}_{. n}=c_{. n}-1 \\
& \bar{c}_{t i}=c_{t i} \quad \quad \text { para } t=1,2, \ldots, T \text { e } i=1,2, \ldots, n-1 \\
& \bar{c}_{T n}=1 .
\end{aligned}
$$

Com essa notação, (3.11) é igual a

$$
\begin{gathered}
\mathbb{E}\left[\left(\sum_{i=1}^{n} \tilde{c}_{. i} b_{i}\right)^{2}\right]+\mathbb{E}\left[\left(\sum_{i=1}^{n} \sum_{t=1}^{T} \bar{c}_{t i} e_{t i}\right)^{2}\right] \\
=\sum_{i=1}^{n} \tilde{c}_{. i}^{2} \operatorname{Var}\left(b_{i}\right)+\sum_{i=1}^{n} \sum_{j=1}^{n} \tilde{c}_{. i} \tilde{c}_{. j} \operatorname{Cov}\left(b_{i}, b_{j}\right)+\sum_{i=1}^{n} \sum_{t=1}^{T} \bar{c}_{t i}^{2} \operatorname{Var}\left(e_{t i}\right) \\
=\frac{(N-1)}{N} S^{2} \sum_{i=1}^{n} \tilde{c}_{. i}^{2}-\frac{S^{2}}{N} \sum_{i=1}^{n} \sum_{j=1}^{n} \tilde{c}_{. i} \tilde{c}_{. j}+\sigma^{2} \sum_{i=1}^{n} \sum_{t=1}^{T} \bar{c}_{t i}^{2} \\
j \neq i \\
=S^{2} \sum_{i=1}^{n} \tilde{c}_{. i}^{2}-\frac{S^{2}}{N}\left(\sum_{i=1}^{n} \tilde{c}_{. i}^{2}+\sum_{i=1}^{n} \sum_{j=1}^{n} \tilde{c}_{. i} \tilde{c}_{. j}\right)+\sigma^{2} \sum_{i=1}^{n} \sum_{t=1}^{T} \bar{c}_{t i}^{2}
\end{gathered}
$$




$$
=S^{2} \sum_{i=1}^{n} \tilde{c}_{. i}^{2}-\frac{S^{2}}{N}\left(\sum_{i=1}^{n} \tilde{c}_{. i}\right)^{2}+\sigma^{2} \sum_{i=1}^{n} \sum_{t=1}^{T} \tilde{c}_{t i}^{2} .
$$

Mas

$$
\begin{gathered}
\sum_{i=1}^{n} \tilde{c}_{. i}=\sum_{i=1}^{n-1} c_{. i}+c_{. n}-1=\sum_{i=1}^{n-1} \sum_{t=1}^{T} c_{t i}+\sum_{t=1}^{T} c_{t n}-1 \\
=\sum_{i=1}^{n} \sum_{t=1}^{T} c_{t i}-1=\sum_{i=1}^{n} \sum_{t=1}^{T-1} c_{t i}+\sum_{i=1}^{n} c_{T i}-1=0
\end{gathered}
$$

e então

$$
\mathbb{E}\left[\mathbf{c}^{\prime} \mathbf{y}-\left(\mu_{T}+b_{n}+e_{T n}\right)\right]^{2}=S^{2} \sum_{i=1}^{n} \tilde{c}_{. i}^{2}+\sigma^{2} \sum_{i=1}^{n} \sum_{t=1}^{T} \bar{c}_{t i}^{2} .
$$

No caso do modelo usualmente utilizado para populações infinitas, onde os $b_{i}$ 's provém de uma distribuição normal e são considerados independentes, a solução do problema levaria à mesma expressão dada por (3.13), com a diferença que $S^{2}$ seria a variância de $b_{i}$. Para populações finitas, de acordo com as definições que adotamos, $\operatorname{Var}\left(b_{i}\right)=\frac{N-1}{N} S^{2}$. Assim, podemos concluir que o resultado que minimizaria (3.13) nos casos finito e infinito é o mesmo, com uma correção no fator $K$. Para populações infinitas, $K=\frac{\operatorname{Var}\left(b_{i}\right)}{\operatorname{Var}\left(e_{t i}\right)}$ enquanto que para populações finitas $K=\frac{N}{N-1} \frac{\operatorname{Var}\left(b_{i}\right)}{\operatorname{Var}\left(e_{t i}\right)}$.

Continuando com estes cálculos, chegaremos aos mesmos resultados já obtidos anteriormente e que estão apresentados na seção B.2 do apêndice B. 


\subsection{CASO 2: MAIS DE UMA OBSERVAÇÃO PERDIDA EM UM SÓ INSTANTE}

Neste seção, estamos estudando o caso em que $m$ observações são perdidas em um dos instantes. Sem perda de generalidade, estamos supondo que os $n-m$ primeiros indivíduos são os que tem os dados completos, e que os últimos $m$ são aqueles que tiveram perdidas suas observações no instante $\mathrm{T}$.

As matrizes $\mathrm{E}$ e $\mathrm{F}$, indicadoras dos dados ausentes e presentes, são compostas por colunas da matriz identidade de dimensão $n T$, adequadamente escolhidas.

Exemplo 3.2 Três indivíduos são observados em três ocasiões e, para dois deles, a última observação é perdida. Então, $n=3, T=3$ e $m=2$

$$
\mathrm{E}=\left(\begin{array}{cc}
0 & 0 \\
0 & 0 \\
0 & 0 \\
\hline 0 & 0 \\
0 & 0 \\
1 & 0 \\
\hline 0 & 0 \\
0 & 0 \\
0 & 1
\end{array}\right) \quad \mathrm{F}=\left(\begin{array}{ccc|cc|cc}
1 & 0 & 0 & 0 & 0 & 0 & 0 \\
0 & 1 & 0 & 0 & 0 & 0 & 0 \\
0 & 0 & 1 & 0 & 0 & 0 & 0 \\
\hline 0 & 0 & 0 & 1 & 0 & 0 & 0 \\
0 & 0 & 0 & 0 & 1 & 0 & 0 \\
0 & 0 & 0 & 0 & 0 & 0 & 0 \\
\hline 0 & 0 & 0 & 0 & 0 & 1 & 0 \\
0 & 0 & 0 & 0 & 0 & 0 & 1 \\
0 & 0 & 0 & 0 & 0 & 0 & 0
\end{array}\right) .
$$

Os resultados completos para o exemplo 3.2 estão no apêndice B.4.

O esquema para o conjunto de dados é: 


\begin{tabular}{|c|c|c|c|}
\hline \multirow[b]{2}{*}{ indiv. } & \multicolumn{2}{|r|}{ tempo } & \multirow[b]{2}{*}{$T$} \\
\hline & 12 & $\ldots T-1$ & \\
\hline 1 & \multirow{4}{*}{\multicolumn{2}{|c|}{$\bar{y}_{c}$}} & \multirow{4}{*}{$\bar{y}_{T}$} \\
\hline 2 & & & \\
\hline$\vdots$ & & & \\
\hline$n-m$ & & & \\
\hline$n-m+1$ & & $(n-m+1)$ & $?$ \\
\hline$n-m+2$ & & $(n-m+2)$ & $?$ \\
\hline$\vdots$ & & $\vdots$ & $\vdots$ \\
\hline$n$ & & $\bar{y}_{n}$ & $?$ \\
\hline
\end{tabular}

onde,

$\bar{y}_{c}=\frac{1}{(n-m)(T-1)} \sum_{t=1}^{T-1} \sum_{i=1}^{n-m} y_{t i}:$ média da parte completa, isto é, média das observações dos $n-m$ indivíduos com dados completos, nos $T-1$ primeiros instantes;

$\bar{y}_{T .}=\frac{1}{n-m} \sum_{i=1}^{n-m} y_{T i}:$ média das observações no instante $T ;$

$\bar{y}_{. i}=\frac{1}{T-1} \sum_{t=1}^{T-1} y_{t i}$, para $i=n-m+1, \ldots, n:$ média das observações do $i-$ ésimo indivíduo (incompleto), nos $T-1$ primeiros instantes.

O BLUP para as observações perdidas, cuja derivação encontra-se no apêndice B.3, é dado por:

$$
\hat{y}_{T i}=\bar{y}_{T .}+\left(\frac{K(T-1)}{K(T-1)+1}\right)\left(\bar{y}_{. i}-\bar{y}_{c}\right), \text { para } i=n-m+1, \ldots, n .
$$

Esse resultado é exatamente igual ao caso de uma única observação ausente, ou seja, é a média no instante $T$ mais uma correção, que depende da parte completa e da média do próprio indivíduo cuja observação está sendo imputada. Este previsor não depende das observações dos outros indivíduos incompletos, o que parece razoável, uma vez que, se a média no instante $T$ depende somente dos indivíduos completos, a correção também deverá depender só deles. 


\subsection{CASO 3: MAIS DE UMA OBSERVAÇÃO PERDIDA EM QUALQUER INSTANTE}

O caso mais geral que estudamos foi o de $m$ observações perdidas em qualquer instante de tempo. Exporemos as idéias através de um exemplo, que iremos mesclando com a formulação do caso geral.

Exemplo 3.3. Consideremos o caso em que quatro indivíduos são observados em quatro instantes, sendo que um deles teve perdidas as observações do segundo e quarto instantes e outro, a observação do primeiro instante, ou seja, teríamos o seguinte esquema de dados:

\begin{tabular}{c|cccc} 
& \multicolumn{5}{|c}{ tempo } \\
indiv. & 1 & 2 & 3 & 4 \\
\hline 1 & $y_{11}$ & $y_{21}$ & $y_{31}$ & $y_{41}$ \\
2 & $y_{12}$ & $y_{22}$ & $y_{32}$ & $y_{42}$ \\
3 & $y_{13}$ & $?$ & $y_{33}$ & $?$ \\
4 & $?$ & $y_{24}$ & $y_{34}$ & $y_{44}$
\end{tabular}

Seja $\mathrm{y}_{a}$ o vetor de dimensão $(m \times 1)$ composto pelos dados ausentes. No exemplo 3.3, $\mathbf{y}_{a}^{\prime}=\left(y_{23}, y_{43}, y_{14}\right)$.

Nosso problema é prever $\mathrm{y}_{a}$, encontrando $\hat{\mathrm{y}}_{a}=\mathrm{Cy}$, onde $\mathrm{C}$ é uma matriz de dimensão $(m \times n T)$.

No exemplo 3.3, 


$$
\mathbf{C}=\left(\begin{array}{llllllllllllll}
c_{111} & c_{121} & c_{131} & c_{141} & c_{112} & c_{122} & c_{132} & c_{142} & c_{113} & c_{123} & c_{133} & c_{143} & c_{114} & c_{124} \\
c_{211} & c_{221} & c_{231} & c_{241} & c_{212} & c_{222} & c_{232} & c_{242} & c_{213} & c_{223} & c_{233} & c_{243} & c_{214} & c_{224} \\
c_{311} & c_{321} & c_{331} & c_{341} & c_{312} & c_{322} & c_{332} & c_{342} & c_{313} & c_{323} & c_{333} & c_{343} & c_{314} & c_{324}
\end{array}\right.
$$

$\left.\begin{array}{ll}c_{134} & c_{144} \\ c_{234} & c_{244} \\ c_{334} & c_{344}\end{array}\right)$

e

$\mathbf{y}^{\prime}=\left(\begin{array}{llllllllllllllll}y_{11} & y_{21} & y_{31} & y_{41} & y_{12} & y_{22} & y_{32} & y_{42} & y_{13} & y_{23} & y_{33} & y_{43} & y_{14} & y_{24} & y_{34} & y_{44}\end{array}\right)$

Queremos encontrar $\mathrm{C}$ que minimize o erro quadrático médio de qualquer combinação linear dos elementos de $\hat{\mathrm{y}}_{a}$, ou seja, queremos determinar $\mathrm{C}$ que minimize

$$
\mathbb{E}\left[\mathrm{l}^{\prime} \mathrm{Cy}-\mathrm{l}^{\prime} \mathrm{y}_{\mathrm{a}}\right]^{2}
$$

para qualquer vetor 1 de constantes de dimensão $(m \times 1)$, sujeito às seguintes restrições:

$$
\begin{gathered}
C E=0 \\
C X=E^{\prime} X
\end{gathered}
$$

e E é a matriz indicadora dos dados ausentes. No exemplo 3.3, estas restrições são:

$$
\left(\begin{array}{lll}
c_{123} & c_{143} & c_{114} \\
c_{223} & c_{243} & c_{214} \\
c_{323} & c_{343} & c_{314}
\end{array}\right)=0 \quad \text { ou } \quad \mathrm{CE}=0
$$


ou seja, as três colunas de $\mathrm{C}$ representadas pelos índices 23,43 e 14 são iguais a zero.

Além disso,

$$
\mathrm{CX}=\left(\begin{array}{cccc}
0 & 1 & 0 & 0 \\
0 & 0 & 0 & 1 \\
1 & 0 & 0 & 0
\end{array}\right)=\mathbf{E}^{\prime} \mathbf{X}
$$

onde

$$
\mathrm{X}=\left(\begin{array}{llll}
1 & 0 & 0 & 0 \\
0 & 1 & 0 & 0 \\
0 & 0 & 1 & 0 \\
0 & 0 & 0 & 1 \\
1 & 0 & 0 & 0 \\
0 & 1 & 0 & 0 \\
0 & 0 & 1 & 0 \\
0 & 0 & 0 & 1 \\
1 & 0 & 0 & 0 \\
0 & 1 & 0 & 0 \\
0 & 0 & 1 & 0 \\
0 & 0 & 0 & 1 \\
1 & 0 & 0 & 0 \\
0 & 1 & 0 & 0 \\
0 & 0 & 1 & 0 \\
0 & 0 & 0 & 1
\end{array}\right) \quad \text { E } \quad \quad \quad\left(\begin{array}{lll}
0 & 0 & 0 \\
0 & 0 & 0 \\
0 & 0 & 0 \\
0 & 0 & 0 \\
0 & 0 & 0 \\
0 & 0 & 0 \\
0 & 0 & 0 \\
0 & 0 & 0 \\
0 & 0 & 0 \\
1 & 0 & 0 \\
0 & 0 & 0 \\
0 & 1 & 0 \\
0 & 0 & 1 \\
0 & 0 & 0 \\
0 & 0 & 0 \\
0 & 0 & 0
\end{array}\right)
$$

Como notação adicional, seja $\operatorname{Cov}\left(\mathrm{y}, \mathrm{y}_{a}^{\prime}\right)=\tilde{\mathrm{W}} \sigma^{2}$ e $\operatorname{Var}\left(\mathrm{y}_{a}\right)=\mathrm{W}_{a} \sigma^{2}$.

As matrizes $\tilde{W}$ e $W_{a}$ são partes da matriz $W$, dada pela expressão (3.4). É complicado obter uma forma fechada para $\tilde{W}$ e $W_{a}$ e por isso as ilustramos através do exemplo. 
Assim, no exemplo 3.3,

$$
\tilde{\mathbf{W}}=\left(\begin{array}{ccc}
-K / N & -K / N & -K / N \\
-K / N & -K / N & -K / N \\
-K / N & -K / N & -K / N \\
-K / N & -K / N & -K / N \\
\hline-K / N & -K / N & -K / N \\
-K / N & -K / N & -K / N \\
-K / N & -K / N & -K / N \\
-K / N & -K / N & -K / N \\
\hline K-K / N & K-K / N & -K / N \\
1+K-K / N & K-K / N & -K / N \\
K-K / N & K-K / N & -K / N \\
K-K / N & 1+K-K / N & -K / N \\
\hline-K / N & -K / N & 1+K-K / N \\
-K / N & -K / N & K-K / N \\
-K / N & -K / N & K-K / N \\
-K / N & -K / N & K-K / N
\end{array}\right)
$$

e

$$
\mathrm{W}_{a}=\left(\begin{array}{ccc}
1+K-K / N & K-K / N & -K / N \\
K-K / N & 1+K-K / N & -K / N \\
-K / N & -K / N & 1+K-K / N
\end{array}\right)
$$

Podemos escrever $\tilde{W}$ e $W_{a}$ em função de W e E. Assim,

$$
\tilde{\mathrm{W}}=\mathrm{WE} \quad \text { e } \quad \mathrm{W}_{a}=\mathrm{E}^{\prime} \mathrm{WE} .
$$

Queremos minimizar (3.15), que é igual a

$$
\begin{aligned}
& \mathbb{E}\left[\mathrm{l}^{\prime} \mathrm{Cy}-\mathrm{l}^{\prime} \mathrm{y}_{a}\right]^{2}=\mathrm{l}^{\prime} \mathbb{E}\left[\left(\mathrm{Cy}-\mathrm{y}_{a}\right)\left(\mathrm{y}^{\prime} \mathrm{C}^{\prime}-\mathrm{y}_{a}^{\prime}\right)\right] \mathrm{l} \\
& =\mathrm{l}^{\prime} \mathrm{CW} \sigma^{2} \mathrm{C}^{\prime} l-\mathrm{l}^{\prime} \mathrm{CW} \sigma^{2} \mathrm{l}-\mathrm{l}^{\prime} \tilde{\mathrm{W}}^{\prime} \sigma^{2} \mathrm{C}^{\prime} l+\mathrm{l}^{\prime} \mathrm{W}_{a} \sigma^{2} \mathrm{l} \\
& =\mathrm{l}^{\prime} \mathrm{CW} \sigma^{2} \mathrm{C}^{\prime} l-\mathrm{l}^{\prime} \mathrm{CWE} \sigma^{2} \mathrm{l}-\mathrm{l}^{\prime} \mathrm{E}^{\prime} \mathrm{W} \sigma^{2} \mathrm{C}^{\prime} l+\mathrm{l}^{\prime} \mathrm{E}^{\prime} \mathrm{WE} \sigma^{2} \mathrm{l}
\end{aligned}
$$


sujeito às restrições (3.16) e (3.17), ou seja, queremos minimizar $f$ dada por:

$f=\mathrm{l}^{\prime} \mathrm{CWC} \mathrm{C}^{\prime} \sigma^{2}-\mathrm{l}^{\prime} \mathrm{CWEl} \sigma^{2}-\mathrm{l}^{\prime} \mathrm{E}^{\prime} \mathrm{WC}^{\prime} \mathrm{l} \sigma^{2}+\mathrm{l}^{\prime} \mathrm{E}^{\prime} \mathrm{WEl} \sigma^{2}+\operatorname{tr}\left(\Lambda_{1} \mathrm{CE}\right)+\operatorname{tr}\left(\Lambda_{2}\left(\mathrm{CX}-\mathrm{E}^{\prime} \mathrm{X}\right)\right)$,

onde $\Lambda_{1}$ e $\Lambda_{2}$ são as matrizes dos multiplicadores de Lagrange, de dimensões $(m \times m)$ e $(T \times m)$, respectivamente.

Usando multiplicadores de Lagrange e os resultados de derivadas de matrizes (ver apêndice A.5), a matriz $\mathrm{C}$ que queremos encontrar é solução do seguinte sistema de equações:

$$
\begin{gathered}
\frac{\partial f}{\partial \mathrm{C}}=21 \mathrm{l}^{\prime} \mathrm{CW} \sigma^{2}-2 \mathrm{ll} \mathrm{E}^{\prime} \mathrm{W} \sigma^{2}+\Lambda_{1}^{\prime} \mathrm{E}^{\prime}+\Lambda_{2}^{\prime} \mathrm{X}^{\prime}=0 \\
\frac{\partial f}{\partial \Lambda_{1}}=\mathrm{E}^{\prime} \mathrm{C}^{\prime}=0 \\
\frac{\partial f}{\partial \Lambda_{2}}=\mathrm{X}^{\prime} \mathrm{C}^{\prime}-\mathrm{X}^{\prime} \mathrm{E}=0 .
\end{gathered}
$$

A matriz C, solução desse sistema, já foi dada no capítulo 2, isto é,

$$
\mathrm{C}=\left\{\mathrm{I}_{m}-\mathrm{E}^{\prime} \mathrm{QE}\right\}^{-1} \mathrm{E}^{\prime} \mathrm{QFF}
$$

onde

$$
\mathrm{Q}=\mathrm{W}^{-1} \mathrm{X}\left(\mathrm{X}^{\prime} \mathrm{W}^{-1} \mathrm{X}\right)^{-1} \mathrm{X}^{\prime} \mathrm{W}^{-1}+\mathrm{I}_{n T}-\mathrm{W}^{-1}
$$

e F é a matriz indicadora dos dados presentes. 
C satisfaz a equação (3.19) pois,

$$
\left.\mathrm{E}^{\prime} \mathrm{C}^{\prime}=\mathrm{E}^{\prime} \mathrm{FF} \mathrm{F}^{\prime} \mathrm{QE} / \mathrm{I}_{m}-\mathrm{E}^{\prime} \mathrm{QE}\right\}^{-1}
$$

Como $\mathrm{E}^{\prime} \mathrm{F}=0$, vale que $\mathrm{E}^{\prime} \mathrm{C}^{\prime}=0$.

Para verificar que $\mathbf{C}$ satisfaz a equação (3.20), basta observar que

$$
\begin{aligned}
\mathrm{CX} & =\left\{\mathrm{I}_{m}-\mathrm{E}^{\prime} \mathrm{QE}\right\}^{-1} \mathrm{E}^{\prime} \mathrm{QFF} \mathrm{F}^{\prime} \mathrm{X} \\
& =\left\{\mathrm{I}_{m}-\mathrm{E}^{\prime} \mathrm{QE}\right\}^{-1} \mathrm{E}^{\prime} \mathrm{Q}\left(\mathrm{I}_{n T}-\mathrm{EE}^{\prime}\right) \mathrm{X} \\
& =\left\{\mathrm{I}_{m}-\mathrm{E}^{\prime} \mathrm{QE}\right\}^{-1}\left(\mathrm{E}^{\prime} \mathrm{QX}-\mathrm{E}^{\prime} \mathrm{QEE} \mathrm{E}^{\prime} \mathrm{X}\right) \\
& =\left\{\mathrm{I}_{m}-\mathrm{E}^{\prime} \mathrm{QE}\right\}^{-1}\left(\mathrm{E}^{\prime} \mathrm{W}^{-1} \mathrm{X}\left(\mathrm{X}^{\prime} \mathbf{W}^{-1} \mathrm{X}\right)^{-1} \mathrm{X}^{\prime} \mathrm{W}^{-1} \mathrm{X}+\mathrm{E}^{\prime} \mathrm{X}-\mathrm{E}^{\prime} \mathrm{W}^{-1} \mathrm{X}-\mathrm{E}^{\prime} \mathrm{QEE} \mathrm{E}^{\prime} \mathrm{X}\right) \\
& =\left\{\mathrm{I}_{m}-\mathrm{E}^{\prime} \mathrm{QE}\right\}^{-1}\left(\mathrm{E}^{\prime} \mathrm{X}-\mathrm{E}^{\prime} \mathrm{QEE} \mathrm{E}^{\prime} \mathrm{X}\right) \\
& =\left\{\mathrm{I}_{m}-\mathrm{E}^{\prime} \mathrm{QE}\right\}^{-1}\left\{\mathrm{I}_{m}-\mathrm{E}^{\prime} \mathrm{QE}\right\} \mathrm{E}^{\prime} \mathrm{X}=\mathrm{E}^{\prime} \mathrm{X}
\end{aligned}
$$

É possível encontrar as matrizes $\Lambda_{1}$ e $\Lambda_{2}$ que satisfazem a equação (3.18). Uma ilustração desse procedimento é dada no apêndice B.5.

Voltando ao exemplo 3.3,

$$
\mathbf{F}=\left(\begin{array}{lllllllllllll}
1 & 0 & 0 & 0 & 0 & 0 & 0 & 0 & 0 & 0 & 0 & 0 & 0 \\
0 & 1 & 0 & 0 & 0 & 0 & 0 & 0 & 0 & 0 & 0 & 0 & 0 \\
0 & 0 & 1 & 0 & 0 & 0 & 0 & 0 & 0 & 0 & 0 & 0 & 0 \\
0 & 0 & 0 & 1 & 0 & 0 & 0 & 0 & 0 & 0 & 0 & 0 & 0 \\
0 & 0 & 0 & 0 & 1 & 0 & 0 & 0 & 0 & 0 & 0 & 0 & 0 \\
0 & 0 & 0 & 0 & 0 & 1 & 0 & 0 & 0 & 0 & 0 & 0 & 0 \\
0 & 0 & 0 & 0 & 0 & 0 & 1 & 0 & 0 & 0 & 0 & 0 & 0 \\
0 & 0 & 0 & 0 & 0 & 0 & 0 & 1 & 0 & 0 & 0 & 0 & 0 \\
0 & 0 & 0 & 0 & 0 & 0 & 0 & 0 & 1 & 0 & 0 & 0 & 0 \\
0 & 0 & 0 & 0 & 0 & 0 & 0 & 0 & 0 & 0 & 0 & 0 & 0 \\
0 & 0 & 0 & 0 & 0 & 0 & 0 & 0 & 0 & 1 & 0 & 0 & 0 \\
0 & 0 & 0 & 0 & 0 & 0 & 0 & 0 & 0 & 0 & 0 & 0 & 0 \\
0 & 0 & 0 & 0 & 0 & 0 & 0 & 0 & 0 & 0 & 0 & 0 & 0 \\
0 & 0 & 0 & 0 & 0 & 0 & 0 & 0 & 0 & 0 & 1 & 0 & 0 \\
0 & 0 & 0 & 0 & 0 & 0 & 0 & 0 & 0 & 0 & 0 & 1 & 0 \\
0 & 0 & 0 & 0 & 0 & 0 & 0 & 0 & 0 & 0 & 0 & 0 & 1
\end{array}\right)
$$




$$
\begin{gathered}
\mathrm{Q}=\frac{1}{n}\left(\mathbf{J}_{n} \otimes \mathbf{I}_{T}\right)+a\left(\mathbf{I}_{n} \otimes \mathbf{J}_{T}\right)-\frac{a}{n}\left(\mathbf{J}_{n} \otimes \mathbf{J}_{T}\right), \\
\text { com } a=\frac{K}{T K+1} \\
\mathbf{E}^{\prime} \mathrm{Q}=\left(\begin{array}{cccc|cccc|cccc|cccc}
a_{1} & a_{2} & a_{1} & a_{1} & a_{1} & a_{2} & a_{1} & a_{1} \\
a_{1} & a_{1} & a_{1} & a_{2} & a_{3} & a_{4} & a_{3} & a_{3} \\
a_{1} & a_{1} & a_{1} & a_{2} & a_{1} & a_{3} & a_{3} & a_{3} & a_{4} & a_{1} \\
a_{2} & a_{1} & a_{1} & a_{1} & a_{2} & a_{1} & a_{1} & a_{1} & a_{1} & a_{1} & a_{2} \\
a_{2} & a_{1} & a_{1} & a_{1} & a_{4} & a_{3} & a_{3} & a_{3}
\end{array}\right)
\end{gathered}
$$

onde $a_{1}=-\frac{a}{n}, \quad a_{2}=\frac{1-a}{n}, \quad a_{3}=\frac{a(n-1)}{n}$ e $a_{4}=\frac{a(n-1)}{n}+\frac{1}{n}$.

$$
\begin{aligned}
& \mathrm{E}^{\prime} \mathrm{QE}=\left(\begin{array}{cc|c}
\frac{a(n-1)}{n}+\frac{1}{n} & \frac{a(n-1)}{n} & -\frac{a}{n} \\
\frac{a(n-1)}{n} & \frac{a(n-1)}{n}+\frac{1}{n} & -\frac{a}{n} \\
-\frac{a}{n} & -\frac{a}{n} & \frac{a(n-1)}{n}+\frac{1}{n}
\end{array}\right) \\
& \mathrm{I}_{3}-\mathrm{E}^{\prime} \mathrm{Q} \mathrm{E}=\frac{n-1}{n}\left(\begin{array}{cc|c}
(1-a) & -a & \frac{a}{n-1} \\
-a & (1-a) & \frac{a}{n-1} \\
\hline \frac{a}{n-1} & \frac{a}{n-1} & (1-a)
\end{array}\right)
\end{aligned}
$$

$A$ inversa de $\mathrm{I}_{3}-\mathrm{E}^{\prime} \mathrm{QE}$ é dada por:

$$
\frac{d}{(n-1)^{2}}\left(\begin{array}{ccc}
(1-a)^{2}(n-1)^{2}-a^{2} & a^{2}+a(1-a)(n-1)^{2} & -a(n-1) \\
a^{2}+a(1-a)(n-1)^{2} & (1-a)^{2}(n-1)^{2}-a^{2} & -a(n-1) \\
-a(n-1) & -a(n-1) & (1-2 a)(n-1)^{2}
\end{array}\right)
$$


onde $d=\frac{n}{n-1}\left[(1-a)^{3}-\frac{2 a^{3}}{(n-1)^{2}}-\frac{2 a^{2}(1-a)}{(n-1)^{2}}-a^{2}(1-a)\right]^{-1}$, ou seja,

$$
d=\frac{12(4 K+1)^{2}}{52 K^{2}+45 K+9}
$$

$\mathrm{E}^{\prime} \mathrm{QFF}=\left(\begin{array}{cccc|cccc|cccc|cccc}a_{1} & a_{2} & a_{1} & a_{1} & a_{1} & a_{2} & a_{1} & a_{1} & a_{3} & 0 & a_{3} & 0 & 0 & a_{2} & a_{1} & a_{1} \\ a_{1} & a_{1} & a_{1} & a_{2} & a_{1} & a_{1} & a_{1} & a_{2} & a_{3} & 0 & a_{3} & 0 & 0 & a_{1} & a_{1} & a_{2} \\ a_{2} & a_{1} & a_{1} & a_{1} & a_{2} & a_{1} & a_{1} & a_{1} & a_{2} & 0 & a_{1} & 0 & 0 & a_{3} & a_{3} & a_{3}\end{array}\right)$

O previsor (BLUP) de $\mathrm{y}_{a}$ é dado por combinações lineares dos dados presentes, cujos coeficientes são apresentados a seguir.

Seja

$$
h=\frac{1}{52 K^{2}+45 K^{\prime}+9}
$$

i) para prever $y_{23}$

$$
\begin{array}{ll}
c_{111}=c_{112}=-4 K(3 K+1) h & c_{113}=8 K(3 K+1) h \\
c_{121}=c_{122}=\frac{1}{3}\left(56 K^{2}+45 K+9\right) h & c_{124}=\frac{1}{3}\left(44 K^{2}+45 K+9\right) h \\
c_{131}=c_{132}=-K\left(8 K^{\prime}+3\right) h & c_{133}=K\left(28 K^{\prime}+9\right) h \\
c_{134}=-3 K(4 K+1) h & c_{141}=c_{142}=\frac{4}{3} K^{2} h \\
c_{144}=-\frac{8}{3} K^{2} h &
\end{array}
$$


ii) para prever $y_{43}$

$$
\begin{array}{ll}
c_{211}=c_{212}=-4 K^{\prime}(3 K+1) h & c_{213}=8 K(3 K+1) h \\
c_{221}=c_{222}=\frac{4}{3} K^{2} h & c_{224}=-\frac{8}{3} K h \\
c_{231}=c_{232}=-K^{\prime}(8 K+3) h & c_{233}=K(28 K+9) h \\
c_{234}=-3 K(4 K+1) h & c_{241}=c_{242}=\frac{1}{3}\left(56 K^{2}+45 K+9\right) h \\
c_{244}=\frac{1}{3}\left(44 K^{2}+45 K+9\right) h &
\end{array}
$$

iii) para prever $y_{14}$

$$
\begin{array}{lll}
c_{311}=c_{312}=\left(20 K^{2}+15 K+3\right) h & c_{313}=3\left(4 K^{2}+5 K^{\prime}+1\right) h \\
c_{321}=c_{322}=-4 K(2 K+1) h & c_{324}=8 K^{\prime}(2 K+1) h \\
c_{331}=c_{332}=-K(4 K+3) h & c_{333}=-3 K^{\prime}\left(4 K^{\prime}+1\right) h \\
c_{334}=K(20 K+9) h & c_{341}=c_{342}=-4 K(2 K+1) h \\
c_{344}=8 K(2 K+1) h &
\end{array}
$$

É difícil obter uma interpretação e uma forma geral para esses coeficientes, uma vez que eles dependem da inversa de $I_{m}-E^{\prime} \mathrm{QE}$.

Para efeitos computacionais, a matriz $\mathrm{C}$ dos coeficientes do previsor (expressão 3.21) pode ser simplificada. Usaremos o seguinte exemplo para explicar como fazê-lo.

Exemplo 3.4. Considere $n=3, T=3$ e $m=5$, de acordo com o seguinte esquema:

\begin{tabular}{c|rcc} 
& \multicolumn{3}{|c}{ tempo } \\
indiv. & 1 & 2 & 3 \\
\hline 1 & $y_{11}$ & $?$ & $y_{31}$ \\
2 & $?$ & $?$ & $y_{32}$ \\
3 & $?$ & $y_{23}$ & $?$
\end{tabular}


Nesse caso,

$$
\mathbf{E}=\left(\begin{array}{lllll}
0 & 0 & 0 & 0 & 0 \\
1 & 0 & 0 & 0 & 0 \\
0 & 0 & 0 & 0 & 0 \\
\hline 0 & 1 & 0 & 0 & 0 \\
0 & 0 & 1 & 0 & 0 \\
0 & 0 & 0 & 0 & 0 \\
\hline 0 & 0 & 0 & 1 & 0 \\
0 & 0 & 0 & 0 & 0 \\
0 & 0 & 0 & 0 & 1
\end{array}\right) \quad \mathbf{F}\left(\begin{array}{llll}
1 & 0 & 0 & 0 \\
0 & 0 & 0 & 0 \\
0 & 1 & 0 & 0 \\
\hline 0 & 0 & 0 & 0 \\
0 & 0 & 0 & 0 \\
0 & 0 & 1 & 0 \\
\hline 0 & 0 & 0 & 0 \\
0 & 0 & 0 & 1 \\
0 & 0 & 0 & 0
\end{array}\right)
$$

No caso geral, a matriz $Q$ pode ser escrita como a soma

$$
\mathrm{Q}=\mathrm{I}_{n T}+\mathrm{P}
$$

Assim,

$$
\begin{gathered}
\mathrm{C}=\left(\mathbf{I}_{m}-\mathrm{E}^{\prime} \mathrm{QE}\right)^{-1} \mathrm{E}^{\prime} \mathrm{QFF} F^{\prime} \\
=\left(\mathbf{I}_{m}-\mathbf{E}^{\prime}\left(\mathbf{I}_{n T}+\mathrm{P}\right) \mathrm{E}\right)^{-1} \mathrm{E}^{\prime}\left(\mathbf{I}_{n T}+\mathbf{P}\right) \mathbf{F F}^{\prime} \\
=\left(-\mathbf{E}^{\prime} \mathrm{PE}\right)^{-1} \mathrm{E}^{\prime} \mathbf{P} \mathbf{F} F^{\prime}
\end{gathered}
$$

pois $E^{\prime} E=I_{m}$ e $E^{\prime} F=0$.

No exemplo 3.4, a matriz -E'PE é dada por

$$
\left(\begin{array}{c|cc|cc}
(n-1)(1-a) & a & -(1-a) & a & a \\
\hline a & (n-1)(1-a) & -(n-1) a & -(1-a) & a \\
-(1-a) & -(n-1) a & (n-1)(1-a) & a & a \\
\hline a & -(1-a) & a & (n-1)(1-a) & -(n-1) a \\
a & a & a & -(n-1) a & (n-1)(1-a)
\end{array}\right)
$$

onde $a=\frac{K}{T K+1}$, 
ou seja, no caso geral, a matriz - E'PE, de dimensão $(m \times m)$, é composta por blocos, cada bloco correspondendo a um dos indivíduos com dado perdido, onde

\section{1) na diagonal}

os blocos da diagonal são matrizes do tipo

$$
(n-1)\left(\begin{array}{cccc}
(1-a) & -a & \cdots & -a \\
-a & (1-a) & \cdots & -a \\
\vdots & \vdots & \vdots & \vdots \\
-a & -a & \cdots & (1-a)
\end{array}\right)
$$

cuja dimensão é igual ao número de observações perdidas daquele indivíduo;

\section{2) fora da diagonal}

fora da diagonal, os elementos são

$$
\begin{aligned}
& -(1-a) \text { se o cruzamento da linha e coluna da matriz - E'PE corresponde } \\
& \text { a tempos iguais } \\
& \text { a se o cruzamento da linha e coluna da matriz - E'PE corresponde } \\
& \text { a tempos diferentes }
\end{aligned}
$$

Assim, no exemplo 3.4, o primeiro bloco da diagonal, correspondente ao primeiro indivíduo, tem dimensão 1 porque esse indivíduo só tem um dado perdido; os segundo e terceiro blocos têm dimensão $(2 \times 2)$, pois cada um dos indivíduos tem dois dados perdidos.

Fora da diagonal, a primeira linha da matriz (3.26) corresponde à observação $y_{21}$ que é uma observação perdida no segundo instante. Quando é cruzada com a terceira coluna de $-\mathbf{E}^{\prime} \mathbf{P E}$, o valor é $-(1-a)$, pois a terceira coluna também corresponde a uma observação perdida no segundo instante; as outras são a, pois essas colunas correspondem 
às observações perdidas em instantes diferentes (no caso, primeiro e terceiro instantes). Por outro lado, no exemplo $3.4, \mathrm{E}^{\prime} \mathrm{PFF}^{\prime}$ tem a seguinte forma:

$$
\mathbf{E}^{\prime} \mathbf{P} \mathbf{F} \mathbf{F}^{\prime}=\left(\begin{array}{ccc|ccc|ccc}
(n-1) a & 0 & (n-1) a & 0 & 0 & -a & 0 & (1-a) & 0 \\
\hline(1-a) & 0 & -a & 0 & 0 & (n-1) a & 0 & -a & 0 \\
-a & 0 & -a & 0 & 0 & (n-1) a & 0 & (1-a) & 0 \\
\hline(1-a) & 0 & -a & 0 & 0 & -a & 0 & (n-1) a & 0 \\
-a & 0 & (1-a) & 0 & 0 & (1-a) & 0 & (n-1) a & 0
\end{array}\right)
$$

ou, na forma geral, cada linha, que corresponde a um dos dados perdidos, será composta por:

\section{1) para os dados presentes}

$(n-1) a, \quad$ se o indivíduo corresponde àquela linha

$(1-a), \quad$ se o indivíduo é diferente do daquela linha; mesmo instante de tempo

$-a, \quad$ se o indivíduo é diferente do daquela linha; instantes de tempo diferentes;

\section{2) para dados ausentes}

0, para qualquer indivíduo e qualquer instante de tempo.

Considerar os coeficientes dados por $\mathrm{E}^{\prime} \mathrm{PFF}^{\prime}$, onde os coeficientes das observações perdidas são zero, nos leva aos mesmos resultados se considerarmos, também para os dados ausentes, o esquema de dados presentes (3.28) e assumirmos que as observações perdidas são iguais a zero.

Essa última alternativa facilita os cálculos computacionais, pois ao invés de trabalharmos com matrizes de dimensão $n T$, passamos a trabalhar com matrizes de dimensão $m$, em geral, muito menores. 
Assim, o BLUP das observações ausentes é dado por:

$$
\left(-\mathrm{E}^{\prime} \mathrm{PE}\right)^{-1} \mathrm{u}
$$

onde u é um vetor de dimensão $(m \times 1)$, cada linha correspondendo a uma das $m$ observações ausentes.

Para a observação perdida do $k$-ésimo indivíduo no $j$-ésimo instante, o elemento de $u$ é dado por

$$
u_{j k}=(1-a) \sum_{i=1}^{n} y_{j i}+a\left((n-1) \sum_{t=1}^{T} y_{t k}-\sum_{\substack{i=1 \\ i \neq k}}^{n} \sum_{t=1}^{t} y_{t i}\right)
$$

onde $a=\frac{K}{T K+1}$,

e que pode ser interpretado como a média no instante com dado perdido mais uma correção que depende da diferença entre a média do indivíduo cujo dado foi perdido e a média dos outros indivíduos nos outros instantes.

\subsection{UMA ILUSTRAÇÃO NUMÉRICA}

Os dados que utilizamos como ilustração são dados reais da pesquisa realizada mensalmente pela Federação das Indústrias do Estado de São Paulo, denominada "Levantamento de Conjuntura - Índices FIESP".

A FIESP conta com um painel fixo de indústrias, que respondem mensalmente a um questionário com dados como total de vendas, número de pessoas ocupadas, número de horas trabalhadas na produção e total de salários. Os questionários são respondidos por 
vários setores produtivos da indústria. Os dados que utilizamos para ilustração são os de número de horas trabalhadas na produção da Indústria de Papel e Papelão do Estado de São Paulo, para empresas com mais de 500 empregados.

Segundo informações da RAIS (Relação Anual de Informações Sociais), esse estrato contém 31 empresas, 13 das quais pertencem ao painel da FIESP.

A tabela 3.1 abaixo mostra os dados dos meses de abril a julho de 1994, disponíveis em 20/08/94, que é a data limite para o fechamento dos resultados de julho. Como pode-se verificar, a empresa 8 não havia fornecido a informação de julho e a empresa 13 , as de abril e julho. Posteriormente, os dados da empresa 13 foram recuperados. Nosso objetivo é obter o BLUP desses dados perdidos.

TABELA 3.1: DADOS

IND.PAPEL E PAPELÃO DO ESTADO DE SÃO PAULO

(Estrato: empresa com mais de 500 empregados)

HORAS TRABALHADAS NA PRODUÇÃO (em milhares)

\begin{tabular}{|l|r|r|r|r|}
\hline & \multicolumn{4}{|c|}{ MESES/94 } \\
\cline { 2 - 5 } & ABR & MAI & JUN & JUL \\
\hline EMPRESA 1 & 327,4 & 374,2 & 349,2 & 359,1 \\
EMPRESA 2 & 304,1 & 305,8 & 302,4 & 297,7 \\
EMPRESA 3 & 235,1 & 218,7 & 221,1 & 233,0 \\
EMPRESA 4 & 243,0 & 253,6 & 244,2 & 245,3 \\
EMPRESA 5 & 235,9 & 229,5 & 218,9 & 211,0 \\
EMPRESA 6 & 129,0 & 144,6 & 132,0 & 130,4 \\
EMPRESA 7 & 164,6 & 113,6 & 113,7 & 146,1 \\
EMPRESA 8 & 171,4 & 167,5 & 169,9 & \\
EMPRESA 9 & 80,0 & 79,8 & 82,1 & 86,1 \\
EMPRESA 10 & 127,3 & 138,8 & 144,7 & 145,1 \\
EMPRESA 11 & 85,4 & 88,5 & 86,3 & 82,9 \\
EMPRESA 12 & 61,2 & 65,7 & 67,6 & 65,7 \\
EMPRESA 13 & & 85,7 & 81,9 & \\
\hline
\end{tabular}

Fonte: FIESP 
Admitimos, neste capítulo, que as componentes da variância são conhecidas. No capítulo 5 tratamos da estimação da variância e mostramos como os valores abaixo propostos podem ser obtidos.

Assim,

$$
\begin{gathered}
\sigma_{H}^{2}=134,611 \quad S_{H}^{2}=9052,093 \\
K=\frac{S_{H}^{2}}{\sigma_{H}^{2}}=67,246 \quad a=\frac{K}{T K^{\prime}+1}=0,249 .
\end{gathered}
$$

-E'PE, dada por (3.26) é:

$$
-\mathrm{E}^{\prime} \mathrm{PE}=\left(\begin{array}{rrr}
9,012 & 0,249 & -0,751 \\
0,249 & 9,012 & -2,988 \\
-0,751 & -2,988 & 9,012
\end{array}\right)
$$

e u, dado por (3.31),

$$
u=\left(\begin{array}{r}
1496,332 \\
553,868 \\
391,868
\end{array}\right)
$$

A previsão para as observaçôes perdidas, dada por (3.30), ou seja, $\left(-\mathrm{E}^{\prime} \mathrm{PE}\right)^{-1} \mathrm{u}$ é igual a

$$
\left(\begin{array}{r}
170,8 \\
85,2 \\
86,0
\end{array}\right)
$$

O valor 170,8 é a previsão do dado da empresa 8 em julho. 85,2 e 86,0 são as previsões da empresa 13 em abril e julho, respectivamente.

No capítulo 6 voltaremos a este exemplo, onde faremos maiores comentários. 


\section{CAPÍTULO 4}

\section{CASO MULTIVARIADO}

\subsection{INTRODUÇÃO}

As idéias desenvolvidas no capítulo 3 podem ser estendidas para o caso multivariado, onde $p$ características são medidas para $n$ indivíduos sorteados de uma população finita de tamanho $N$, em $T$ ocasiões diferentes.

Estamos considerando somente o caso de não-resposta total, isto é, o caso em que as $p$ observações do questionário foram perdidas. A não-resposta parcial (perda de parte do questionário) não faz parte do escopo deste trabalho.

O modelo que utilizamos é a extensão, para $p$ variáveis, do modelo univariado, isto é, 


$$
\mathrm{y}_{t i}=\mu+\alpha_{t}+\mathrm{b}_{i}+\mathrm{e}_{t i} \quad \begin{aligned}
& t=1,2, \ldots, T \\
& i=1,2, \ldots, n
\end{aligned}
$$

onde

$\mathrm{y}_{t i}^{\prime}=\left(y_{1 t i}, y_{2 t i}, \ldots, y_{p t i}\right):$ vetor de observações do indivíduo $i$ no instante $t$

$\boldsymbol{\mu}^{\prime}=\left(\mu_{1}, \mu_{2}, \ldots, \mu_{p}\right)$ : média geral ;

$\alpha_{t}^{\prime}=\left(\alpha_{1 t}, \alpha_{2 t}, \ldots, \alpha_{p t}\right)$ : vetor fixo medindo o efeito do tempo ;

$\mathbf{b}_{i}^{\prime}=\left(\mathrm{b}_{1 i}, \mathrm{~b}_{2 i}, \ldots, \mathrm{b}_{p i}\right)$ : vetor aleatório medindo o efeito do indivíduo ;

$\mathbf{e}_{t i}^{\prime}=\left(\mathbf{e}_{1 t i}, \mathbf{e}_{2 t i}, \ldots, \mathbf{e}_{p t i}\right):$ vetor de erros associado ao individuo $i$ no instante $t$.

As suposições são as mesmas,

$$
\begin{gathered}
\mathrm{e}_{t i} \sim N_{p}(0, \Sigma) \\
\operatorname{com} \quad \Sigma=\left(\begin{array}{cccc}
\sigma_{11} & \sigma_{12} & \cdots & \sigma_{1 p} \\
\sigma_{21} & \sigma_{22} & \cdots & \sigma_{2 p} \\
\vdots & \vdots & \vdots & \vdots \\
\sigma_{p 1} & \sigma_{p 2} & \cdots & \sigma_{p p}
\end{array}\right)
\end{gathered}
$$

e

$\mathrm{e}_{t i}$ 's independentes;

$\mathbf{e}_{t i}$ 's e $\mathrm{b}_{i}$ 's independentes.

Definimos

$$
\begin{aligned}
& \sum_{i=1}^{T} \alpha_{j t}=0 \quad \text { para qualquer } j=1,2, \ldots, p \\
& \sum_{i=1}^{N} b_{j i}=0 \quad \text { para qualquer } j=1,2, \ldots, p
\end{aligned}
$$

e 


$$
S_{j j^{\prime}}=\frac{1}{N-1} \sum_{i=1}^{N} b_{j i} b_{j^{\prime} i} \quad \text { para quaisquer } j, j^{\prime}=1,2, \ldots, p .
$$

O vetor y é o vetor composto por todas as observações, isto é,

$$
\mathrm{y}^{\prime}=\left(y_{111}, y_{211}, \ldots, y_{p 11}, \ldots, y_{1 T 1}, y_{2 T 1}, \ldots, y_{p T 1}, \ldots, y_{11 n}, y_{21 n}, \ldots\right.
$$

$$
\left.\ldots, y_{p 1 n}, \ldots, y_{1 T n}, y_{2 T n}, \ldots, y_{p T n}\right)
$$

$\operatorname{com} \mathbb{E}(\mathrm{y})=\boldsymbol{\mu} \quad$ e $\operatorname{Var}(\mathrm{y})=\mathrm{V}$.

Seja

$$
\mathbf{S}=\left(\begin{array}{cccc}
S_{11} & S_{12} & \cdots & S_{1 p} \\
S_{21} & S_{22} & \cdots & S_{2 p} \\
\vdots & \vdots & \vdots & \vdots \\
S_{p 1} & S_{p 2} & \cdots & S_{p p}
\end{array}\right)
$$

Estamos supondo que $\Sigma$ e S são de posto completo. Observando que, para quaisquer $j, j^{\prime}=1,2, \ldots, p$,

i)

$$
\begin{aligned}
0=\left(\sum_{i=1}^{N} b_{j i}\right)\left(\sum_{i^{\prime}=1}^{N} b_{j^{\prime} i^{\prime}}\right) & =\sum_{i=1}^{N} b_{j i} b_{j^{\prime} i}+\sum_{i=1}^{N} \sum_{\substack{i^{\prime}=1 \\
i^{\prime} \neq i}}^{N} b_{j i} b_{j^{\prime} i^{\prime}}=(N-1) S_{j j^{\prime}}+\sum_{\substack { i=1 \\
\begin{subarray}{c}{i^{\prime}=1 \\
i^{\prime} \neq i{ i = 1 \\
\begin{subarray} { c } { i ^ { \prime } = 1 \\
i ^ { \prime } \neq i } }\end{subarray}}^{N} \sum_{j i} b_{j^{\prime} i^{\prime}} \\
& \Longrightarrow \sum_{\substack { i=1 \\
\begin{subarray}{c}{i^{\prime}=1 \\
i^{\prime} \neq i{ i = 1 \\
\begin{subarray} { c } { i ^ { \prime } = 1 \\
i ^ { \prime } \neq i } }\end{subarray}}^{N} \sum_{j i} b_{j^{\prime} i^{\prime}}=-(N-1) S_{j j^{\prime}}
\end{aligned}
$$

ii)

$$
\mathbb{E}\left(b_{j i}\right)=\frac{1}{N} \sum_{i=1}^{N} b_{j i}=0
$$




$$
\begin{aligned}
& \operatorname{Cov}\left(b_{j i}, b_{j^{\prime} i}\right)=\mathbb{E}\left(b_{j i} b_{j^{\prime} i}\right)=\frac{1}{N} \sum_{i=1}^{N} b_{j i} b_{j^{\prime} i}=\left(1-N^{-1}\right) S_{j j^{\prime}} \\
& \operatorname{Cov}\left(b_{j i}, b_{j^{\prime} i^{\prime}}\right)=\mathbb{E}\left(b_{j i} b_{j^{\prime} i^{\prime}}\right)=\frac{1}{N(N-1)} \sum_{i=1}^{N} \sum_{\substack{i^{\prime}=1 \\
i^{\prime} \neq i}}^{N} b_{j i} b_{j^{\prime} i^{\prime}}=-\frac{S_{j j^{\prime}}}{N},
\end{aligned}
$$

podemos calcular as covariâncias entre os elementos do vetor y. Assim,

$$
\mathrm{V}=\operatorname{Var}(\mathrm{y})=\left(\mathrm{I}_{n} \otimes \mathbf{I}_{T} \otimes \mathbf{\Sigma}\right)+\left(\mathbf{I}_{n} \otimes \mathrm{J}_{T} \otimes \mathrm{S}\right)-\frac{1}{N}\left(\mathbf{J}_{n} \otimes \mathbf{J}_{T} \otimes \mathbf{S}\right)
$$

ou seja,

$$
\mathrm{V}=\left(\begin{array}{cccc}
\mathrm{C}+\mathrm{B} & \mathrm{C} & \cdots & \mathrm{C} \\
\mathrm{C} & \mathrm{C}+\mathrm{B} & \cdots & \mathrm{C} \\
\vdots & \vdots & \vdots & \vdots \\
\mathrm{C} & \mathrm{C} & \cdots & \mathrm{C}+\mathrm{B}
\end{array}\right)
$$

onde $\quad \mathrm{C}=-\frac{1}{N}\left(\begin{array}{cccc}\mathrm{S} & \mathrm{S} & \cdots & \mathrm{S} \\ \mathrm{S} & \mathrm{S} & \cdots & \mathrm{S} \\ \vdots & \vdots & \vdots & \vdots \\ \mathrm{S} & \mathrm{S} & \cdots & \mathrm{S}\end{array}\right)$ e $\mathrm{B}=\left(\begin{array}{cccc}\mathrm{S}+\Sigma & \mathrm{S} & \cdots & \mathrm{S} \\ \mathrm{S} & \mathrm{S}+\Sigma & \cdots & \mathrm{S} \\ \vdots & \vdots & \vdots & \vdots \\ \mathrm{S} & \mathrm{S} & \cdots & \mathrm{S}+\Sigma\end{array}\right)$.

Como no caso univariado, a matriz $\mathrm{C}+\mathrm{B}$ reflete as covariâncias "dentro do indivíduo", enquanto que a matriz $\mathrm{C}$ reflete as covariâncias "entre indivíduos", devidas ao sorteio sem reposição.

Note que três tipos de covariâncias estão envolvidas neste processo:

- a covariância entre as $p$ variáveis;

- a covariância devida ao fato das medidas serem repetidas no tempo, por tratar-se de um painel e

- a covariância devida ao plano amostral para a população finita. 
No modelo para populações infinitas, a matriz $\mathrm{V}$ é dada por:

$$
\mathrm{V}_{\infty}=\left(\mathrm{I}_{n} \otimes \mathrm{J}_{T} \otimes \mathrm{S}\right)+\left(\mathrm{I}_{n} \otimes \mathrm{I}_{T} \otimes \mathbf{\Sigma}\right)
$$

ou seja,

$$
\mathrm{V}_{\infty}=\left(\begin{array}{cccc}
\mathrm{B} & 0 & \cdots & 0 \\
0 & \mathrm{~B} & \cdots & 0 \\
\vdots & \vdots & \vdots & \vdots \\
0 & 0 & \cdots & \mathrm{B}
\end{array}\right)
$$

A matriz inversa de $\mathrm{V}$ é:

$$
\mathrm{V}^{-1}=\left(\mathrm{I}_{n} \otimes \mathrm{I}_{T} \otimes \mathrm{\Sigma}^{-1}\right)-\left(\mathrm{I}_{n} \otimes \mathrm{J}_{T} \otimes \mathrm{H}\right)+\left(\mathrm{J}_{n} \otimes \mathrm{J}_{T} \otimes \mathrm{G}\right)
$$

onde

$$
\begin{gathered}
\mathrm{H}=(T \mathrm{~S}+\Sigma)^{-1} \mathbf{S} \Sigma^{-1} \\
\mathbf{G}=\frac{1}{N}(T(1-f) \mathrm{S}+\Sigma)^{-1} \Sigma(T \mathrm{~S}+\Sigma)^{-1} \mathbf{S} \Sigma^{-1}
\end{gathered}
$$

e $f=\frac{n}{N} \quad$ (fração amostral).

A demonstração desse resultado encontra-se na seção C.1 do apêndice C.

Para esse modelo, as matrizes $\mathrm{X}$ e $\mathrm{Z}$ são dadas por: 


$$
\mathrm{X}=\left(\begin{array}{ccc}
\mathrm{I}_{p} & 0 & 0 \\
0 & \mathrm{I}_{p} & 0 \\
\vdots & \vdots & \vdots \\
0 & 0 & \mathrm{I}_{p} \\
\mathrm{I}_{p} & 0 & 0 \\
0 & \mathrm{I}_{p} & 0 \\
\vdots & \vdots & \vdots \\
0 & 0 & \mathrm{I}_{p} \\
\mathrm{I}_{p} & 0 & 0 \\
0 & \mathrm{I}_{p} & 0 \\
\vdots & \vdots & \vdots \\
0 & 0 & \mathrm{I}_{p}
\end{array}\right) \quad \mathrm{Z}=\left(\begin{array}{ccc}
\mathrm{I}_{p} & 0 & 0 \\
\mathrm{I}_{p} & 0 & 0 \\
\vdots & \vdots & \vdots \\
\mathrm{I}_{p} & 0 & 0 \\
0 & \mathrm{I}_{p} & 0 \\
0 & \mathrm{I}_{p} & 0 \\
\vdots & \vdots & \vdots \\
0 & \mathrm{I}_{p} & 0 \\
0 & 0 & \mathrm{I}_{p} \\
0 & 0 & \mathrm{I}_{p} \\
\vdots & \vdots & \vdots \\
0 & 0 & \mathrm{I}_{p}
\end{array}\right)
$$

ou

$$
\mathrm{X}=\mathbf{1}_{n} \otimes \mathrm{I}_{T} \otimes \mathrm{I}_{p} \quad \text { e } \quad \mathrm{Z}=\mathrm{I}_{n} \otimes \mathrm{I}_{T} \otimes \mathrm{I}_{p}
$$

e

$$
\left(\mathrm{X}^{\prime} \mathrm{V}^{-1} \mathrm{X}\right)^{-1}=\frac{1}{n}\left[\left(\mathrm{I}_{T} \otimes \Sigma\right)-\left(\mathrm{J}_{T} \otimes\left(T n^{2} \mathrm{G}-n T \mathrm{H}+n \Sigma^{-1}\right)^{-1}\left(n^{2} \mathrm{G}-n \mathrm{H}\right) \Sigma\right]\right.
$$

onde $\mathbf{H}$ e $\mathbf{G}$ são dados pelas expressões (4.6) e (4.7), respectivamente.

A matriz Q, dada por,

$$
\mathrm{Q}=\mathrm{V}^{-1} \mathrm{X}\left(\mathrm{X}^{\prime} \mathrm{V}^{-1} \mathrm{X}\right)^{-1} \mathrm{X}^{\prime} \mathrm{V}^{-1}+\mathrm{I}_{n} T_{p}-\mathrm{V}^{-1}
$$

é igual a

$$
\mathrm{Q}=\left(\mathrm{I}_{n} \otimes \mathrm{I}_{T} \otimes \mathrm{I}_{p}\right)+\left(\frac{1}{n} \mathrm{~J}_{n}-\mathrm{I}_{n}\right) \otimes\left(\mathrm{I}_{T} \otimes \Sigma^{-1}-\mathrm{J}_{T} \otimes \mathrm{H}\right)
$$


Note que Q não depende de $N$, o tamanho da população, ou de $f$, a fração amostral. A demonstração das expressões (4.8) e (4.9) podem ser encontradas na seção C.2 do apêndice C.

\subsection{CASO 1: UMA OBSERVAÇÃO PERDIDA NO ÚLTIMO INSTANTE}

Uma única observação perdida significa, neste trabalho, a perda de um questionário completo e não a perda da observação de uma das variáveis do questionário. Assim, podemos supor, sem perda de generalidade, que a observação perdida corresponde ao último indivíduo no último instante e teríamos a seguinte situaçào no conjunto de dados:

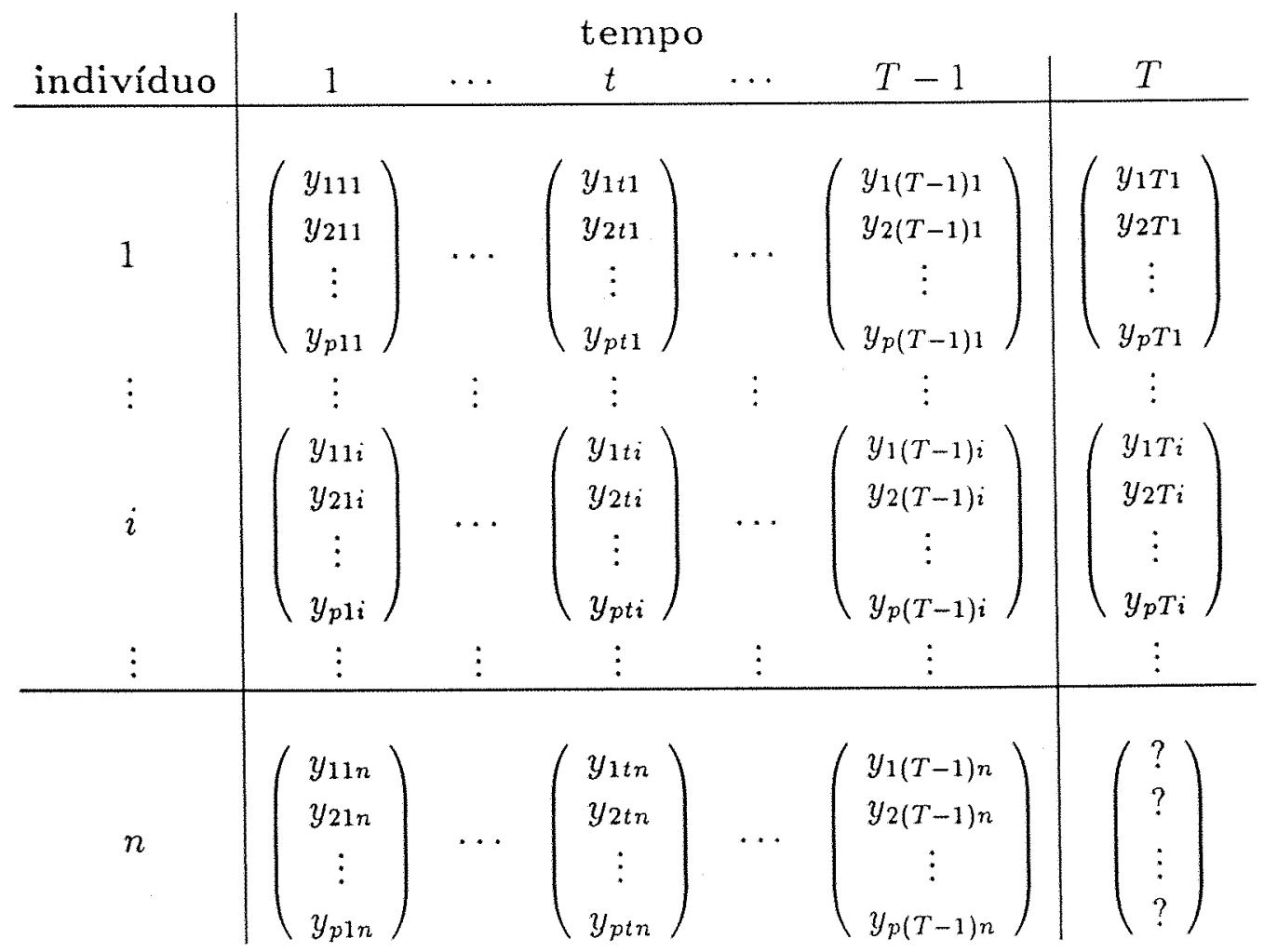

$\mathrm{O}$ nosso problema é prever o vetor $\mathrm{y}_{T n}$ de dimensão $(p \times 1)$. Utilizamos os multipli- 
cadores de Lagrange pois os cálculos são mais simples do que se usássemos a expressão (2.39). Queremos obter $p$ combinações lineares dos elementos do vetor $\mathrm{y}$, isto é, queremos obter

$$
\sum_{t=1}^{T} \sum_{i=1}^{n} \mathrm{C}_{t i} \mathrm{y}_{t i}
$$

onde $\mathrm{C}_{t i}$ é uma matriz de constantes de dimensão $(p \times p)$, com $\mathrm{C}_{T n}=0$, tal que

$$
\mathbb{E}\left[\sum_{t=1}^{T} \sum_{i=1}^{n} \mathrm{C}_{t i} \mathrm{y}_{t i}\right]=\boldsymbol{\mu}_{T}
$$

o que implica em

i) para $t=1,2, \ldots, T-1, \quad \sum_{i=1}^{n} \mathrm{C}_{t i}=0$

ii) $\sum_{i=1}^{n-1} \mathrm{C}_{T i}=\mathrm{I}_{p} \quad$ e $\quad \sum_{i=1}^{n} \mathrm{C}_{T i}=\mathrm{I}_{p}$

e tal que minimize

$$
\mathbb{E}\left[\left(\mathrm{l}^{\prime} \sum_{t=1}^{T} \sum_{i=1}^{n} \mathrm{C}_{t i} \mathrm{y}_{t i}-\mathrm{l}^{\prime}\left(\mu_{T}+\mathrm{b}_{n}+\mathbf{e}_{T n}\right)\right)^{2}\right],
$$

para qualquer vetor 1 de constantes de dimensão $(p \times 1)$.

$$
\begin{array}{rlr}
\text { Seja } & \tilde{\mathrm{C}}_{. i}=\mathrm{C}_{. i}=\sum_{t=1}^{T} \mathrm{C}_{t i} & \text { para } i=1,2, \ldots, n-1 \\
\tilde{\mathrm{C}}_{. n} & =\mathrm{C}_{. n}-\mathrm{I}_{p}=\sum_{t=1}^{T} \mathrm{C}_{t n}-\mathrm{I}_{p} & \\
\overline{\mathrm{C}}_{t i} & =\mathrm{C}_{t i} & \text { para } t=1,2, \ldots, T \text { e } i=1,2, \ldots, n-1 \\
\overline{\mathrm{C}}_{T n} & =\mathrm{I}_{p} . & \text { ou para } t=1,2, \ldots, T-1 \mathrm{e} i=1,2, \ldots, n
\end{array}
$$


Minimizar (4.10) é equivalente a minimizar

$$
\mathrm{l}^{\prime}\left[\sum_{i=1}^{n} \tilde{\mathrm{C}}_{. i} \mathrm{~S} \tilde{\mathrm{C}}_{. i}^{\prime}-\frac{1}{N}\left(\sum_{i=1}^{n} \tilde{\mathrm{C}}_{. i}\right) \mathrm{S}\left(\sum_{i=1}^{n} \tilde{\mathrm{C}}_{. i}\right)^{\prime}+\sum_{i=1}^{n} \sum_{t=1}^{T} \overline{\mathrm{C}}_{t i} \Sigma \overline{\mathrm{C}}_{t i}^{\prime}\right] \mathrm{l}
$$

(ver seção C.3 do apêndice C).

Mas,

$$
\begin{gathered}
\sum_{i=1}^{n} \tilde{\mathrm{C}}_{. i}=\sum_{i=1}^{n-1} \mathrm{C}_{. i}+\mathrm{C}_{. n}-\mathrm{I}_{p}=\sum_{i=1}^{n-1} \sum_{t=1}^{T} \mathrm{C}_{t i}+\sum_{t=1}^{T} \mathrm{C}_{t n}-\mathrm{I}_{p} \\
=\sum_{i=1}^{n} \sum_{t=1}^{T} \mathrm{C}_{t i}-\mathrm{I}_{p}=\sum_{i=1}^{n} \sum_{t=1}^{T-1} \mathrm{C}_{t i}+\sum_{i=1}^{n} \mathrm{C}_{T i}-\mathrm{I}_{p}=0
\end{gathered}
$$

Assim, queremos minimizar

$$
\mathrm{l}^{\prime} \sum_{i=1}^{n} \tilde{\mathrm{C}}_{. i} \mathrm{~S} \tilde{\mathrm{C}}_{. i}^{\prime} 1+\mathrm{l}^{\prime} \sum_{i=1}^{n} \sum_{t=1}^{T} \overline{\mathrm{C}}_{t i} \Sigma \overline{\mathrm{C}}_{t i}^{\prime} \mathrm{l}
$$

que leva, como no caso univariado, ao mesmo resultado para populações infinitas, com uma pequena correção.

Voltando à notação original, queremos minimizar $f$,

$$
\begin{aligned}
f= & \mathrm{l}^{\prime} \sum_{i=1}^{n}\left(\sum_{t=1}^{T} \mathrm{C}_{t i}\right) \mathrm{S}\left(\sum_{t=1}^{T} \mathrm{C}_{t i}\right)^{\prime} \mathrm{l}-\mathrm{l}^{\prime}\left(\sum_{t=1}^{T} \mathrm{C}_{t n}\right) \mathrm{Sl} \\
& -\mathrm{l}^{\prime} \mathrm{S}\left(\sum_{t=1}^{T} \mathrm{C}_{t n}\right)^{\prime} \mathrm{l}+\mathrm{l}^{\prime} \mathrm{S} \mathrm{l}+\mathrm{l}^{\prime} \sum_{i=1}^{n-1} \sum_{t=1}^{T-1} \mathrm{C}_{t i} \Sigma \mathrm{C}_{t i}^{\prime} \mathrm{l} \\
& +\mathrm{l}^{\prime} \sum_{i=1}^{n-1} \mathrm{C}_{T i} \Sigma \mathrm{C}_{T i}^{\prime} \mathrm{l}+\mathrm{l}^{\prime} \sum_{t=1}^{T-1} \mathrm{C}_{t n} \Sigma \mathrm{C}_{t n}^{\prime} \mathrm{l}+\mathrm{l}^{\prime} \Sigma \mathrm{l}
\end{aligned}
$$




$$
+\sum_{t=1}^{T-1} \operatorname{tr}\left(\Lambda_{t} \sum_{i=1}^{n} \mathrm{C}_{t i}\right)+\operatorname{tr}\left(\Lambda_{T}\left(\sum_{i=1}^{n-1} \mathrm{C}_{T i}-\mathrm{I}_{p}\right)\right)+\operatorname{tr}\left(\Lambda_{T+1} \mathrm{C}_{T n}\right)
$$

onde as matrizes $\Lambda_{t}, t=1,2, \ldots, T+1$ são as matrizes, de dimensão $(p \times p)$, dos multiplicadores de Lagrange.

O sistema de equações, gerado pelas derivadas de $f$, é formado pelas seguintes equações:

1) $\frac{\partial f}{\partial \mathrm{C}_{T j}}=21 \mathrm{l}^{\prime} \mathrm{C}_{T j} \mathrm{~S}+2 \mathrm{ll} \mathrm{l}^{\prime}\left(\sum_{t=1}^{T-1} \mathrm{C}_{t j}\right) \mathrm{S}+21 \mathrm{l}^{\prime} \mathrm{C}_{T j} \Sigma+\Lambda_{T}^{\prime}$

$$
=211^{\prime} \sum_{t=1}^{T} \mathrm{C}_{t j} \mathrm{~S}+21 \mathrm{l}^{\prime} \mathrm{C}_{T j} \Sigma+\Lambda_{T}^{\prime}=0
$$

$(n-1$ equações correspondentes a $t=T$ e $j=1,2, \ldots, n-1)$

2) $\frac{\partial f}{\partial \mathrm{C}_{v n}}=21 \mathrm{l}^{\prime} \mathrm{C}_{v n} \mathrm{~S}+\mathrm{ll}^{\prime}\left(\sum_{\substack{t=1 \\ t \neq v}}^{T} \mathrm{C}_{t n}\right) \mathrm{S}+1 \mathrm{l}^{\prime}\left(\sum_{\substack{t=1 \\ t \neq v}}^{T} \mathrm{C}_{t n}\right) \mathrm{S}$

$$
-1 l^{\prime} \mathrm{S}-1 \mathrm{ll}^{\prime} \mathrm{S}+21 \mathrm{l}^{\prime} \mathrm{C}_{v n} \Sigma+\Lambda_{v}^{\prime}
$$$$
=21 l^{\prime} \mathrm{C}_{v n} \mathrm{~S}+2 \mathrm{ll}^{\prime}\left(\sum_{\substack{t=1 \\ t \neq v}}^{T} \mathrm{C}_{t n}\right) \mathrm{S}-2 \mathrm{ll}^{\prime} \mathrm{S}+2 \mathrm{ll}^{\prime} \mathrm{C}_{v n} \Sigma+\Lambda_{v}^{\prime}
$$$$
=21 l^{\prime}\left(\sum_{t=1}^{T} \mathrm{C}_{t n}\right) \mathrm{S}-21 \mathrm{l}^{\prime} \mathrm{S}+21 \mathrm{l}^{\prime} \mathrm{C}_{v n} \Sigma+\Lambda_{v}^{\prime}=0
$$

$(T-1$ equações correspondentes a $i=n$ e $v=1,2, \ldots, T-1)$ 
3) $\frac{\partial f}{\partial \mathrm{C}_{v j}}=211^{\prime} \mathrm{C}_{v j} \mathrm{~S}+2 \mathrm{ll}^{\prime}\left(\sum_{\substack{t=1 \\ t \neq v}}^{T} \mathrm{C}_{t j}\right) \mathrm{S}+211^{\prime} \mathrm{C}_{v j} \Sigma+\Lambda_{v}^{\prime}$

$$
=2 \mathrm{Il}^{\prime}\left(\sum_{t=1}^{T} \mathrm{C}_{t j}\right) \mathrm{S}+2 \mathrm{Il}^{\prime} \mathrm{C}_{v j} \Sigma+\Lambda_{v}^{\prime}=0
$$

$((n-1)(T-1)$ equações correspondentes a $j=1,2, \ldots, n-1$ e $v=1,2, \ldots, T-1)$

4) $\frac{\partial f}{\partial \Lambda_{v}}=\left(\sum_{i=1}^{n} \mathrm{C}_{v i}\right)^{\prime}=0$

$(T-1)$ equações correspondentes a $v=1,2, \ldots, T-1)$

5) $\frac{\partial f}{\partial \Lambda_{T}}=\left(\sum_{i=1}^{n-1} \mathrm{C}_{T_{i}}-\mathrm{I}_{p}\right)^{\prime}=0$

6) $\frac{\partial f}{\partial \Lambda_{T+1}}=\mathrm{C}_{T_{n}}^{\prime}=0$.

A solução desse sistema, cuja verificação encontra-se na seção C.4 do apêndice C, é a seguinte:

$$
\begin{aligned}
& \mathrm{C}_{T i}=\frac{1}{n-1} \mathrm{I}_{p}, \quad i=1,2, \ldots, n-1 \\
& \mathrm{C}_{t n}=\mathrm{S}(\mathrm{S}(T-1)+\Sigma)^{-1}, \quad t=1,2, \ldots, T-1 \\
& \mathrm{C}_{t i}=-\frac{1}{n-1} \mathrm{~S}(\mathrm{~S}(T-1)+\Sigma)^{-1}, \quad i=1,2, \ldots, n-1 \text { e } t=1,2, \ldots, T-1 \\
& \mathrm{C}_{T_{n}}=0, \\
& \Lambda_{t}=0, \quad t=1,2, \ldots, T-1
\end{aligned}
$$




$$
\begin{aligned}
& \Lambda_{T}=-\frac{2}{n-1} \mathrm{ll}^{\prime}\left(-(T-1) \mathrm{S}(\mathrm{S}(T-1)+\Sigma)^{-1} \mathrm{~S}+\mathrm{S}+\Sigma\right), \\
& \boldsymbol{\Lambda}_{T+1}=0 .
\end{aligned}
$$

Assim, o BLUP de $y_{T n}$ é

$$
\hat{\mathrm{y}}_{T_{n}}=\overline{\mathrm{y}}_{T .}+(T-1) \mathrm{S}(\mathrm{S}(T-1)+\Sigma)^{-1}\left(\overline{\mathrm{y}}_{. n}-\overline{\mathrm{y}}_{c}\right)
$$

onde

$$
\begin{aligned}
\overline{\mathrm{y}}_{c}=\frac{1}{(n-1)(T-1)} \sum_{t=1}^{T-1} \sum_{i=1}^{n-1} \mathrm{y}_{t i}: & \text { vetor de médias da parte completa dos dados (média } \\
& \text { das observações dos } n-1 \text { indivíduos completos } \\
& \text { nos } T-1 \text { primeiros instantes) }
\end{aligned}
$$

$\bar{y}_{T .}=\frac{1}{n-1} \sum_{i=1}^{n-1} y_{T i}:$ vetor de médias das observações no instante $\mathrm{T}$;

$\overline{\mathrm{y}}_{. n}=\frac{1}{T-1} \sum_{t=1}^{T-1} \mathrm{y}_{t n}:$ vetor de médias das observações do indivíduo $\mathrm{n}$.

\subsection{CASO 2: $m$ OBSERVAÇÕES PERDIDAS EM QUALQUER INSTANTE}

Para o caso multivariado, $m$ observações perdidas significa a perda de $m$ questionários completos. Estamos considerando que essas $m$ observações podem ser de qualquer indivíduo, em qualquer instante. A obtenção do BLUP é análoga ao caso univariado, isto é, 


$$
\mathrm{C}=\left\{\mathrm{I}_{m p}-\mathrm{E}^{\prime} \mathrm{QE}\right\}^{-1} \mathrm{E}^{\prime} \mathrm{QFF}
$$

onde Q é dado pela expressão (4.9) e E e F são as matrizes indicadoras dos dados ausentes e presentes.

Para exemplificar, consideraremos um exemplo análogo ao exemplo 3.4 do capítulo 3 , só que neste caso $\mathrm{y}_{t i}$ é um vetor de dimensão $(p \times 1)$, correspondente às $p$ variáveis observadas.

Exemplo 4.1. Considere $n=3, T=3$ e $m=5$, de acordo com o seguinte esquema:

\begin{tabular}{c|ccc} 
& \multicolumn{3}{|c}{ tempo } \\
indiv. & 1 & 2 & 3 \\
\hline 1 & $\mathrm{y}_{11}$ & $?$ & $\mathrm{y}_{31}$ \\
2 & $?$ & $?$ & $\mathrm{y}_{32}$ \\
3 & $?$ & $\mathrm{y}_{23}$ & $?$
\end{tabular}

Neste caso,

$$
\mathrm{E}=\left(\begin{array}{ccccc}
0 & 0 & 0 & 0 & 0 \\
\mathrm{I}_{p} & 0 & 0 & 0 & 0 \\
0 & 0 & 0 & 0 & 0 \\
\hline 0 & \mathrm{I}_{p} & 0 & 0 & 0 \\
0 & 0 & \mathrm{I}_{p} & 0 & 0 \\
0 & 0 & 0 & 0 & 0 \\
\hline 0 & 0 & 0 & \mathrm{I}_{p} & 0 \\
0 & 0 & 0 & 0 & 0 \\
0 & 0 & 0 & 0 & \mathrm{I}_{p}
\end{array}\right) \quad \mathrm{F}=\left(\begin{array}{cccc}
\mathrm{I}_{p} & 0 & 0 & 0 \\
0 & 0 & 0 & 0 \\
0 & \mathrm{I}_{p} & 0 & 0 \\
\hline 0 & 0 & 0 & 0 \\
0 & 0 & 0 & 0 \\
0 & 0 & \mathrm{I}_{p} & 0 \\
\hline 0 & 0 & 0 & 0 \\
0 & 0 & 0 & \mathrm{I}_{p} \\
0 & 0 & 0 & 0
\end{array}\right)
$$


No caso geral,

$$
\mathrm{Q}=\mathrm{I}_{n T p}+\mathrm{P}
$$

onde

$$
\mathbf{P}=\left(\begin{array}{cccc}
-(n-1) & 1 & \cdots & 1 \\
1 & -(n-1) & \cdots & 1 \\
\vdots & \vdots & \vdots & \vdots \\
1 & 1 & \cdots & -(n-1)
\end{array}\right) \otimes\left(\begin{array}{cccc}
\mathrm{A} & \mathrm{B} & \cdots & \mathrm{B} \\
\mathrm{B} & \mathrm{A} & \cdots & \mathrm{B} \\
\vdots & \vdots & \vdots & \vdots \\
\mathrm{B} & \mathrm{B} & \cdots & \mathrm{A}
\end{array}\right)
$$

$\mathbf{A}=\frac{1}{n}\left(\Sigma^{-1}-(T S+\Sigma)^{-1} S \Sigma^{-1}\right)$

e $\mathbf{B}=\frac{1}{n}(T S+\Sigma)^{-1} \mathbf{S} \Sigma^{-1}$.

No exemplo 4.1,

$-\mathbf{E}^{\prime} \mathbf{P E}=\left(\begin{array}{r|rr|rr}(n-1) \mathbf{A} & \mathbf{B} & -\mathbf{A} & \mathbf{B} & \mathbf{B} \\ \hline \mathbf{B} & \begin{array}{r}(n-1) \mathbf{A} \\ -(n-1) \mathbf{B}\end{array} & -\mathbf{A} & \mathbf{B} \\ -\mathbf{A} & -(n-1) \mathbf{B} & (n-1) \mathbf{A} & \mathbf{B} & \mathbf{B} \\ \hline \mathbf{B} & -\mathbf{A} & \mathbf{B} & (n-1) \mathbf{A} & -(n-1) \mathbf{B} \\ \mathbf{B} & \mathbf{B} & \mathbf{B} & -(n-1) \mathbf{B} & (n-1) \mathbf{A}\end{array}\right)$

e

$$
\mathbf{E}^{\prime} \mathbf{P F F}^{\prime}=\left(\begin{array}{rrr|rrr|rrr}
(n-1) \mathbf{B} & 0 & (n-1) \mathbf{B} & 0 & 0 & -\mathbf{B} & 0 & \mathbf{A} & 0 \\
\hline \mathbf{A} & 0 & -\mathbf{B} & 0 & 0 & (n-1) \mathbf{B} & 0 & -\mathbf{B} & 0 \\
-\mathbf{B} & 0 & -\mathbf{B} & 0 & 0 & (n-1) \mathbf{B} & 0 & \mathbf{A} & 0 \\
\hline \mathbf{A} & 0 & -\mathbf{B} & 0 & 0 & -\mathbf{B} & 0 & (n-1) \mathbf{B} & 0 \\
-\mathbf{B} & 0 & \mathbf{A} & 0 & 0 & \mathbf{A} & 0 & (n-1) \mathbf{B} & 0
\end{array}\right) .
$$


As regras para construção das matrizes $-E^{\prime} \mathrm{PE}$ e $\mathrm{E}^{\prime} \mathbf{P} \mathrm{FF}^{\prime}$ são análogas às regras do caso univariado, basta substituir $(1-a)$ e a (caso univariado) por A e B (caso multivariado), respectivamente.

Como no caso univariado, o BLUP das observações perdidas é dado por:

$$
\left(-\mathrm{E}^{\prime} \mathrm{PE}\right)^{-1} \mathrm{u}
$$

onde $\mathbf{E}^{\prime} \mathrm{PE}$ tem dimensão $(m p \times m p)$ e u é um vetor de dimensão $(m p \times 1)$, tal que, para a observação ausente do $k$-ésimo indivíduo no $j$-ésimo instante, o elemento de u é

$$
\mathrm{u}_{j k}=\mathrm{A} \sum_{i=1}^{n} \mathrm{y}_{j i}+\mathrm{B}\left((n-1) \sum_{t=1}^{T} \mathrm{y}_{t k}-\sum_{\substack{i=1 \\ i \neq k}}^{n} \sum_{\substack{t=1 \\ i \neq j}}^{T} \mathrm{y}_{t i}\right)
$$

\subsection{UMA ILUSTRAÇÃO NUMÉRICA}

Para ilustrar a obtenção do BLUP no caso multivariado, voltamos ao exemplo numérico exposto na seção 3.5 do capítulo 3. Nesta seção estamos utilizando os dados de horas trabalhadas na produção, já apresentados na tabela 3.1, e os de total de pessoal ocupado, apresentados na tabela 4.1 a seguir. 
TABELA 4.1: DADOS

IND.PAPEL E PAPELÃO DO ESTADO DE SÃO PAULO

(Estrato: empresa com mais de 500 empregados)

TOTAL DE PESSOAL OCUPADO

\begin{tabular}{|l|r|r|r|r|}
\hline & \multicolumn{4}{|c|}{ MESES/94 } \\
\cline { 2 - 5 } & ABR & MAI & JUN & JUL \\
\hline EMPRESA 1 & 2703 & 2691 & 2670 & 2648 \\
EMPRESA 2 & 2330 & 2323 & 2312 & 2280 \\
EMPRESA 3 & 2089 & 2083 & 2086 & 2080 \\
EMPRESA 4 & 1761 & 1757 & 1737 & 1655 \\
EMPRESA 5 & 1729 & 1698 & 1654 & 1652 \\
EMPRESA 6 & 1095 & 1089 & 1075 & 1044 \\
EMPRESA 7 & 970 & 969 & 963 & 938 \\
EMPRESA 8 & 864 & 861 & 854 & \\
EMPRESA 9 & 815 & 818 & 822 & 848 \\
EMPRESA 10 & 766 & 761 & 770 & 767 \\
EMPRESA 11 & 722 & 723 & 719 & 732 \\
EMPRESA 12 & 518 & 508 & 516 & 513 \\
EMPRESA 13 & & 548 & 544 & 28 \\
\hline
\end{tabular}

Fonte: FIESP

Novamente, admitimos que as variâncias e covariâncias são conhecidas. Posteriormente, no capítulo 5 , mostraremos como os valores abaixo propostos podem ser obtidos. Então,

$$
\begin{gathered}
\Sigma=\left(\begin{array}{rr}
134,611 & 36,378 \\
36,378 & 399,084
\end{array}\right) \\
S=\left(\begin{array}{rr}
9052,093 & 68.116,493 \\
68.116,493 & 539.133,254
\end{array}\right) .
\end{gathered}
$$

$-E^{\prime} \mathrm{PE}$, dada por (4.18), 


$$
-\mathrm{E}^{\prime} \mathrm{PE}=10^{-6}\left(\begin{array}{rrrrrr}
1781 & -496 & 49 & -12 & -148 & 41 \\
-496 & 5393 & -12 & 136 & 41 & -449 \\
49 & -12 & 1781 & -496 & -591 & 145 \\
-12 & 136 & -496 & 5393 & 145 & -1637 \\
-148 & 41 & -591 & 145 & 1781 & -496 \\
41 & -449 & 145 & -1637 & -496 & 5393
\end{array}\right)
$$

u, dado por (4.21),

$$
\mathbf{u}=\left(\begin{array}{c}
1.359880 \\
0,467555 \\
0,691186 \\
0,126537 \\
0,461709 \\
0,095978
\end{array}\right)
$$

e a previsão dos dados ausentes

$$
\left(\begin{array}{r}
837,0 \\
165,7 \\
557,4 \\
83,7 \\
526,8 \\
84,1
\end{array}\right)
$$

A tabela 4.2 abaixo mostra os dados das empresas, com os valores já imputados.

TABELA 4.2: DADOS COMPLETADOS

IND.PAPEL E PAPELÃO DO ESTADO DE SÃO PAULO

(Estrato: empresa com mais de 500 empregados)

\begin{tabular}{|c|l|r|r|r|r|}
\hline \multicolumn{2}{|c|}{} & \multicolumn{4}{|c|}{ MESES/94 } \\
\cline { 3 - 6 } \multicolumn{2}{|c|}{ EMPRESA 8 8} & ABR & MAI & JUN & JUL \\
& & & & & \\
& PESSOAL & 864 & 861 & 854 & $\mathbf{8 3 7 , 0}$ \\
& HORAS & 171,4 & 167,5 & 169,9 & $\mathbf{1 6 5 , 7}$ \\
EMPRESA 13 13 & PESSOAL & $\mathbf{5 5 7 , 4}$ & 548 & 544 & $\mathbf{5 2 6 , 8}$ \\
& HORAS & $\mathbf{8 3 , 7}$ & 85,7 & 81,9 & $\mathbf{8 4 , 1}$ \\
\hline
\end{tabular}

Fonte: FIESP

No capítulo 6 voltaremos a este exemplo e mostraremos uma tabela com os resultados deste previsor e de mais outros dois. 


\section{CAPÍTULO 5}

\section{O CASO DE VARIÂNCIAS DESCONHECIDAS}

\subsection{INTRODUÇÃO}

Nos capítulos anteriores obtivemos o BLUP dos efeitos fixos, aleatórios e das observações perdidas, supondo que as variâncias fossem conhecidas. No caso univariado, não era necessário conhecer cada uma das componentes de variância, mas somente a razão entre elas. No caso multivariado, supusemos conhecidas as matrizes de covariâncias dos efeitos aleatórios e erros.

Quando esses valores são desconhecidos, o método tradicional é primeiro estimar as componentes de variância e então proceder como se estes valores fossem os verdadeiros. Esse procedimento é conhecido como BLUP empírico e foi usado, por exemplo, por Ware (1985). Kackar e Harville (1981) mostraram que esse procedimento resulta em previso- 
res não-viesados das combinações lineares dos efeitos, desde que o vetor de dados seja simetricamente distribuido em torno de seu valor esperado e desde que o estimador das componentes de variância sejam invariantes por translação e sejam funções pares do vetor de dados.

Esse procedimento pode apresentar problemas quando a estimativa da razão das variâncias é próxima de zero. Segundo Harville e Carriquiry (1992), " empirical BLUP is satisfactory - or can be made satisfactory by introducing appropriate modifications - unless the estimate of the variance ratio is imprecise and is close to zero, in which case more sensible point and interval predictions can be obtained by adopting a Bayesian approach".

Quando as componentes de variância são desconhecidas e substituídas por valores estimados, o erro quadrático médio dos previsores dos efeitos cresce. Kackar e Harville (1984) estudaram a magnitude desse crescimento e propuseram uma aproximação geral sob a suposição de nomalidade de erros e efeitos.

Há considerável literatura sobre a estimação das componentes de variância. Uma vasta bibliografia sobre o assunto é dada por Sahai, Khuri e Kapadia (1985).

Até 1970, basicamente dois procedimentos eram utilizados: um deles considerando funções quadráticas, sem fazer qualquer suposição sobre a distribuição das variáveis, e usando técnicas de Análise de Variância; outro usando técnicas de estimação de máxima verossimilhança, assumindo a distribuição normal. Uma variante do estimador de máxima verossimilhança é o estimador de máxima verossimilhança restrito, conhecido como REML (Restricted Estimator Maximum Likelihood).

No início da década de 70, C.R. Rao propôs um método mais geral para obter o estimador quadrático não-viesado de norma mínima. Esse método é conhecido como Teoria MINQUE (Minimum Norm Quadratic Unbiased Estimation). Adiante, dedicaremos uma 
seção ao REML e ao MINQUE, onde daremos referências a respeito.

O problema das técnicas que utilizam a ANOVA é que podem ser encontradas estimativas negativas para valores essencialmente positivos. La Motte (1973b) caracterizou as combinações lineares das componentes da variância para as quais existe estimador quadrático não-viesado não-negativo. Ele mostrou que a componente correspondente ao erro, no modelo misto da ANOVA, é a única que pode ter esse tipo de estimador, ou seja, não existe estimador quadrático não-viesado não-negativo da variância dos efeitos aleatórios.

Vários métodos têm sido sugeridos. Alguns autores estudaram estimadores nãonegativos, que em geral são estimadores truncados e viesados. Hartung (1981) considerou estimadores invariantes com respeito à translação da média, com vício mínimo e caracterizou, entre eles, o estimador de norma mínima, que sempre existe. Chow e Shao (1988) desenvolveram estimadores não-negativos para o modelo de componentes de variância com um fator aleatório, assumindo normalidade e independência dos erros e efeitos e observaram que seu método pode ser usado para modelos de efeitos aleatórios mais complexos. Mathew, Sinha e Sutradhar (1992) consideraram o modelo misto com duas componentes de variância e obtiveram condições suficientes sob as quais existe um estimador quadrático invariante não-negativo para a variância do fator aleatório, tendo um erro quadrático médio uniformemente menor do que os invariantes quadráticos não-viesados.

As propriedades assintóticas de estimadores foram estudadas por Das (1989), que fez um pequeno estudo de simulação de Monte Carlo para o modelo misto nâo balanceado com dois fatores e interação. Ele considerou distribuição normal e independência para fatores, interação e erro.

Do ponto de vista Bayesiano, o problema foi estudado por Harville (1974), que obteve 
uma representação conveniente para a verossimilhança dada por Patterson e Thompson (1971) no estimador de máxima verossimilhança restrito, Gnot e Kleffe (1983), que obtiveram estimadores quadráticos invariantes para uma combinação linear das componentes de variância e Lee e Kapadia (1992), que, usando a regra de Jeffrey, obtiveram estimadores para o modelo misto de blocos incompletos, comparando-os com os estimadores usuais da ANOVA.

Rao, Kaplan e Cochran (1981) consideraram um modelo de efeitos aleatórios com um fator, com a suposição de normalidade para efeitos e erros, e estudaram oito métodos para estimar as variâncias, todos eles sendo variaçòes do ANOVA, MINQUE e máxima verossimilhança.

Outros métodos considerados na literatura são os métodos introduzidos por Henderson (1953), usados por Wesolowska e Janczarek (1984) e conhecidos como métodos I, II e III de Henderson.

Esses trabalhos consideraram o conjunto de dados completos. A estimação da matriz de covariâncias na presença de dados ausentes é um problema bastante complicado. Jennrich e Schluchter (1986) e Laird, Lange e Stram (1987) propuseram o algoritmo EM para estimar parâmetros em modelos de dados longitudinais. Calvin (1993) propôs uma modificação do EM para o modelo misto geral multivariado e Mensah, Elswick e Chinchilli (1993) construíram quatro estimadores consistentes. Todos eles assumiram a normalidade dos dados.

Apesar de toda essa variedade de métodos considerados na literatura, segundo Searle (1988) as principais maneiras de estimar as variâncias em modelos mistos ou de efeitos aleatórios são: estimador da ANOVA, máxima verossimilhança, máxima verossimilhança restrito e MINQUE. 
Por motivos já citados anteriormente, não usaremos o enfoque de estimação de máxima verossimilhança. Consideraremos a estimação das componentes de variância com base na parte completa dos dados. O estudo da estimação da variância com dados incompletos não é objeto deste trabalho, mas faz parte de planos de futuras pesquisas. Na próxima seção faremos algumas considerações sobre os estimadores da ANOVA, REML e MINQUE para o caso univariado.

\subsection{CASO UNIVARIADO}

\subsubsection{O MÉTODO DE ANÁLISE DE VARIÂNCIA - ANOVA}

A estimação das componentes de variância baseada na Análise de Variância é um dos métodos mais utilizados quando o conjunto de dados é balanceado. Esse método se baseia nos quadrados médios da tabela da Análise de Variância do correspondente modelo de efeitos fixos. O procedimento consiste em construir a tabela da ANOVA para o modelo de efeitos fixos e calcular as esperanças dos quadrados médios envolvidos, sob o modelo de efeitos aleatórios ou misto. Essas esperanças são funções lineares das componentes de variância e daí é possível obter estimadores não-viesados para esses parâmetros.

Os quadrados médios são funções quadráticas dos dados e então, os estimadores das variâncias pelo método da ANOVA são estimadores quadráticos não-viesados. Maiores detalhes sobre o assunto podem ser encontrados em livros de planejamento e modelos lineares como Winer (1971), Searle (1971) e Peres e Saldiva (1982).

O problema dos estimadores obtidos por esse método é que é possivel obter estimativas negativas para as componentes da variância correspondentes aos efeitos aleatórios. Uma 
solução é truncar os estimadores no ponto zero, isto é, o estimador da variância dos erros é o próprio estimador da ANOVA, uma vez que ele é sempre positivo, e o estimador da variância dos efeitos é o estimador obtido na tabelas da ANOVA se este for positivo, e é zero em caso contrário.

Consideremos, então, o modelo dado por (3.1),

$$
y_{t i}=\mu+\alpha_{t}+b_{i}+e_{t i} \quad \begin{aligned}
t & =1,2, \ldots, T \\
i & =1,2, \ldots, n .
\end{aligned}
$$

Esse modelo corresponde ao modelo de planejamento por blocos completos aleatorizados e, como já vimos no capítulo 3, pode ser escrito como

$$
\mathrm{y}=\mathrm{X} \alpha+\mathrm{Zb}+\mathrm{e}
$$

com y dado por (3.3) e

$$
\mathrm{X}=\mathrm{1}_{n} \otimes \mathrm{I}_{T} \quad, \quad \mathrm{Z}=\mathrm{I}_{n} \otimes 1_{T} .
$$

Consideremos agora, as somas de quadrados. A tabela da ANOVA é dada por:

\begin{tabular}{l|c|c|c|c}
\hline Fonte de Variação & SS & G.L. & MS & E(MS) \\
\hline Indivíduo & SSind & $n-1$ & MSind & $\sigma^{2}+T S^{2}$ \\
Tempo & SStem & $T-1$ & MStem & $\sigma^{2}+n S^{2}$ \\
Erro & SSerr & $(n-1)(T-1)$ & MSerr & $\sigma^{2}$ \\
\hline Total & SStot & $n T-1$ & & \\
\hline
\end{tabular}

onde

$$
\operatorname{SSind}=\sum_{i=1}^{n} \sum_{t=1}^{T}\left(\bar{y}_{. i}-\bar{y}_{. .}\right)^{2}=T\left(\sum_{i=1}^{n} \bar{y}_{. i}^{2}-n \bar{y} . .2\right)
$$




$$
\begin{gathered}
\text { SStem }=\sum_{i=1}^{n} \sum_{t=1}^{T}\left(\bar{y}_{t .}-\bar{y}_{. .}\right)^{2}=n\left(\sum_{t=1}^{T} \bar{y}_{t .}^{2}-T \bar{y}_{. .}^{2}\right) \\
\text { SStot }=\sum_{i=1}^{n} \sum_{t=1}^{T}\left(y_{t i}-\bar{y}_{. .}\right)^{2}=\sum_{i=1}^{n} \sum_{t=1}^{T} y_{t i}^{2}-n T \bar{y}_{. .}^{2} \\
\text { SSerr }=\text { SStot }- \text { SSind }- \text { SStem }
\end{gathered}
$$

ou, na forma matricial

$$
\begin{gathered}
\operatorname{SSind}=\frac{1}{T} \mathrm{y}^{\prime}\left(\mathrm{I}_{n T}-\mathrm{X}\left(\mathrm{X}^{\prime} \mathrm{X}\right)^{-1} \mathrm{X}^{\prime}\right) \mathrm{ZZ}^{\prime} \mathrm{y} \\
\text { SSerr }=\mathrm{y}^{\prime}\left(\mathrm{I}_{n T}-\mathrm{X}\left(\mathrm{X}^{\prime} \mathrm{X}\right)^{-1} \mathrm{X}^{\prime}\right)\left(\mathrm{I}_{n T}-\frac{1}{T} \mathrm{ZZ}^{\prime}\right) \mathrm{y} .
\end{gathered}
$$

O cálculo das esperanças das somas de quadrados estão no apêndice D.1 e os estimadores das componentes da variância são:

$$
\hat{\sigma}_{A}^{2}=M S e r r=\frac{1}{(n-1)(T-1)}\left(\sum_{i=1}^{n} \sum_{t=1}^{T} y_{t i}^{2}-n \sum_{t=1}^{T} \bar{y}_{t .}^{2}-T \sum_{i=1}^{n} \bar{y}_{. i}^{2}+n T \bar{y}_{. .}^{2}\right)
$$

e

$$
\hat{S}_{A}^{2}=\frac{1}{T}(M \operatorname{Sind}-M \operatorname{Serr}) .
$$

Se adotarmos a seguinte notação:

$$
A=\sum_{i=1}^{n} \sum_{t=1}^{T}\left(y_{t i}-\bar{y}_{i .}\right)^{2}=\sum_{i=1}^{n} \sum_{t=1}^{T} y_{t i}^{2}-n \sum_{t=1}^{T} \bar{y}_{t .}^{2}
$$

(variação dentro do tempo) e 


$$
B=\sum_{i=1}^{n} \sum_{t=1}^{T}\left(\bar{y}_{. i}-\bar{y}_{. .}\right)^{2}=T\left(\sum_{i=1}^{n} \bar{y}_{. i}^{2}-n \bar{y}_{. .}^{2}\right)
$$

( variação entre a média de indivíduos)

teremos que,

$$
\hat{\sigma}_{A}^{2}=\frac{A-B}{(n-1)(T-1)}
$$

e

$$
\hat{S}_{A}^{2}=\frac{T B-A}{T(n-1)(T-1)} .
$$

\subsubsection{O MÉTODO DE ESTIMAÇÃO DE MÁXIMA VEROSSIMILHANÇA RESTRITA - REML}

Os primeiros pesquisadores a sugerir o uso de técnicas de máxima verossimilhança para a estimação de componentes de variância foram Crump $(1947,1951)$ e Hartley e Rao (1967) baseados na suposição de normalidade dos dados.

A desvantagem do estimador de máxima verossimilhança é que, em geral é viesado e, no caso balanceado, é diferente do estimador da ANOVA que, segundo Graybill e Hultquist (1961) é o melhor estimador quadrático não-viesado em dados balanceados e o melhor não-viesado em dados balanceados com suposição de normalidade.

O problema do viés pode ser superado pelo uso do REML, estimador de máxima verossimilhança restrito ou estimador de máxima verossimilhança residual. Esse nome foi dado ao estimador por causa do uso dos resíduos no procedimento de estimação. 
Essa técnica, inicialmente introduzida para dados balanceados por Anderson e Bancroft (1952), e Thompson Jr. (1962), foi posteriormente estendida por Patterson e Thompson (1971), e talvez essa seja sua principal referência, e por Patterson e Thompson (1975) para dados não balanceados.

Outros autores que pesquisaram o assunto foram Laird e Ware (1982), que descreveram como o algoritmo EM pode ser usado para obter o EMV (estimador de máxima verossimilhança) e o REML, Laird (1982) que introduziu uma classe de EMV's generalizados, que contém tanto o EMV como o REML, Robinson (1987) que descreveu vários métodos para estimar as componentes de variância e Klotz (1990) que obteve o EMV e o REML para a covariância entre efeitos aleatórios e erros para o modelo misto com interação.

A técnica do REML procura maximizar a verossimilhança conjunta de contrastes de erros com esperança zero, uma vez que contrastes sobre efeitos fixos não trazem qualquer informação sobre a estrutura de covariâncias. Este método separa a logaritmo da função de verossimilhança em duas partes, estimando as componentes de variância pela maximização de uma das partes e os efeitos fixos pela maximização da outra.

Para dados balanceados, no caso normal, se o estimador da ANOVA for positivo, ele é idêntico ao REML; caso contrário, o REML é o estimador da ANOVA truncado no zero.

Alguns trabalhos fazem a conexão entre o BLUP e o REML. Entre eles estão Harville (1977), Thompson (1980), Fellner (1986, 1987) e McGilchrist (1994). Segundo Robinson (1991), "REML is the method of estimating variance components that seems to have the best credentials from a classical viewpoint". Speed (1991) acrescenta que, no caso normal, "REML and BLUP are intimately connected. Indeed one view - certainly not the only one - of the REML equations for variance components is that they are simply equating 
observed with expected sums of squares of BLUPs". Isto significa que, sob normalidade, o REML é dado pela solução das seguintes equações:

$$
\begin{gathered}
\sum_{i=1}^{n} \hat{b}_{i}^{2}=\sum_{i=1}^{n} \mathbb{E}\left[\hat{b}_{i}^{2}\right] \\
\sum_{i=1}^{n} \sum_{t=1}^{T} \hat{e}_{t i}^{2}=\sum_{i=1}^{n} \sum_{t=1}^{T} \mathbb{E}\left[\hat{e}_{t i}^{2}\right] .
\end{gathered}
$$

Considerando observações completas e o modelo $y_{t i}=\mu_{t}+b_{i}+e_{t i}$, temos

$$
\begin{gathered}
\hat{b}_{i}=\frac{T S^{2}}{T S^{2}+\sigma^{2}}\left(\bar{y}_{. i}-\bar{y}_{. .}\right)^{2} \\
\hat{\mu}_{t}=\bar{y}_{t .} \\
\hat{e}_{t i}=y_{t i}-\hat{\mu}_{t}-\hat{b}_{i}=\left(y_{t i}-\bar{y}_{t .}\right)-\frac{T S^{2}}{T S^{2}+\sigma^{2}}\left(\bar{y}_{. i}-\bar{y}_{. .}\right) .
\end{gathered}
$$

Calculando $\mathbb{E}\left(\hat{b}_{i}^{2}\right)$ e $\mathbb{E}\left(\hat{e}_{t i}^{2}\right)$, o sistema de equações dado por (5.16) e (5.17), para o modelo que adotamos, é

$$
\begin{gathered}
\frac{T\left(S^{2}\right)^{2}}{\left(T S^{2}+\sigma^{2}\right)^{2}} B=\frac{T(n-1)\left(S^{2}\right)^{2}}{T S^{2}+\sigma^{2}} \\
A+\frac{T^{2}\left(S^{2}\right)^{2}}{\left(T S^{2}+\sigma^{2}\right)^{2}} B-\frac{2 T S^{2}}{T S^{2}+\sigma^{2}} B=\frac{T(n-1) \sigma^{2}}{\left(T S^{2}+\sigma^{2}\right)}\left((T-1) S^{2}+\sigma^{2}\right)
\end{gathered}
$$

onde $A$ e $B$ são os mesmos da seção anterior, dados por (5.12) e (5.13), respectivamente. 
A demonstração dos resultados apresentados nesta seção encontram-se no apêndice D.2.

A solução do sistema acima, obtida por intermédio do pacote computacional Mathematica (Wolfram (1991)), é

$$
\hat{\sigma}^{2}=\frac{A-B}{(n-1)(T-1)} \quad \text { e } \quad \hat{S}^{2}=\frac{T B-A}{T(n-1)(T-1)},
$$

que são os mesmos estimadores obtidos pelo método da ANOVA.

Assim, os estimadores de máxima verossimilhança restritos de $\sigma^{2}$ e $S^{2}$, sob a suposição de normalidade, são

$$
\begin{gathered}
\hat{\sigma}_{R}^{2}=\frac{A-B}{(n-1)(T-1)} \\
\hat{S}_{R}^{2}= \begin{cases}\hat{S}^{2}=\frac{T B-A}{T(n-1)(T-1)} & \text { se } \hat{S}^{2} \geq 0 \\
0 & \text { se } \hat{S}^{2}<0 .\end{cases}
\end{gathered}
$$

A título de observação, sob a suposição de normalidade dos efeitos, os estimadores de máxima verossimilhança das componentes de variância são:

$$
\hat{\sigma}^{2}=\frac{A-B}{n(T-1)} \quad \text { e } \quad \hat{S}^{2}=\frac{T B-A}{n T(T-1)} .
$$

O REML, quando positivo, é o estimador de máxima verossimilhança corrigido para que seja não-viesado. 


\subsubsection{O MÉTODO DE ESTIMAÇÃO QUADRÁTICA NÃO-VIESADA DE NORMA MÍNIMA - MINQUE}

A teoria MINQUE (Minimum Norm Quadratic Unbiased Estimation) foi proposta em 1970 por C.R.Rao, na tentativa de apresentar um método mais geral do que os existentes, para o problema de estimação das componentes de variância. O resultado de sua pesquisa foi uma série de artigos na década de 1970 (Rao (1970, 1971a, 1971b, 1972, 1979)) e a posterior publicação de um livro, cuja referência é Rao e Kleffe (1988). Outros trabalhos dessa época são La Motte (1970) e La Motte (1973a).

Há uma grande variedade de variantes sobre o MINQUE, que podem ser encontradas no livro acima citado, em Chaubey (1984) e Das Peddada (1989). Entre elas podemos citar o MIVQUE (Minimum Variance Quadratic Unbiased Estimation) e o MIMSQE (Minimum Mean Square Quadratic Estimation).

Alguns autores consideraram variações no sentido de obter o MINQUE não-negativo. Relaxando a condição de ser não-viesado, Rao e Chaubey (1978) sugeriram o MINQE. Rao e Subrahmaniam (1971) sugeriram, quando da obtenção de um valor negativo, a sua substituição por um número positivo "pequeno", como por exemplo 0,01 . Chaubey (1983) obteve o CMINQUE ( Closest to MINQUE) e Chaubey (1984) estudou duas outras modificações, uma delas considerando o estimador igual ao MINQUE se positivo, e igual ao CMINQUE se negativo, e a outra igual ao MINQUE se positivo, e igual ao MINQE se negativo.

Mais recentemente, Volaufová e Witkovsky (1992) definiram e obtiveram o MINQUE de funções lineares das componentes de variância no modelo linear misto sob restrições lineares e Heine (1993) considerou o caso não-balanceado e dois procedimentos para estimar 
as componentes de variância. A idéia da teoria MINQUE é obter um estimador quadrático não-viesado que seja próximo do estimador natural dos parâmetros. Para clarear as idéias, consideremos primeiro o modelo linear de Gauss-Markov, de efeitos fixos,

$$
\mathrm{y}=\mathrm{X} \boldsymbol{\beta}+\varepsilon, \quad \varepsilon \sim\left(0, \sigma^{2} \mathrm{I}\right)
$$

onde y é um vetor de dimensão $(d \times 1)$.

Suponha que desejamos estimar uma função linear dos elementos do vetor $\boldsymbol{\beta}, \mathbf{c}^{\prime} \boldsymbol{\beta}$. Se $\varepsilon$ fosse conhecido, o estimador natural de $\mathbf{c}^{\prime} \beta$ seria

$$
\mathrm{a}^{\prime}(\mathrm{y}-\varepsilon)=\mathrm{a}^{\prime} \mathrm{X} \boldsymbol{\beta} \quad \text { com } \mathrm{a}^{\prime} \mathrm{X}=\mathrm{c}^{\prime} .
$$

Como $\varepsilon$ é desconhecido, a idéia é obter o vetor a que minimize $\mathbf{a}^{\prime} \mathbf{y}-\mathbf{c}^{\prime} \boldsymbol{\beta}$, que é igual a $\mathbf{a}^{\prime} \varepsilon$.

Como $\varepsilon$ é uma variável aleatória, podemos minimizar a norma de a , $\|\mathbf{a}\|$, para uma norma adequadamente escolhida, ou seja, queremos obter

$$
\min \left\{\|\mathrm{a}\|^{2}: \mathbf{a}^{\prime} \mathbf{X}=\mathbf{c}^{\prime}\right\}
$$

Se o objetivo for estimar $\sigma^{2}$, a mesma idéia pode ser aplicada. Para $\beta$ conhecido, o estimador natural de $\sigma^{2}$ é

$$
d^{-1} \varepsilon^{\prime} \varepsilon
$$

Queremos obter uma forma quadrática de y,

$$
\mathrm{y}^{\prime} \mathrm{Cy}=(\mathrm{X} \beta+\varepsilon)^{\prime} \mathrm{C}(\mathrm{X} \beta+\varepsilon)
$$


que seja não-viesada para $\sigma^{2}$, e para isso precisamos colocar a restrição de que $\operatorname{tr}(\mathbf{C})=1$, e que seja próxima do estimador natural dado por (5.30). Além disso, $\beta$ é desconhecido, e então, gostaríamos que $y^{\prime} \mathrm{Cy}$ não dependesse de $\beta$. O que garante isso é a restrição $\mathrm{CX}=0$ pois

$$
\mathrm{y}^{\prime} \mathrm{Cy}=\beta^{\prime} \mathrm{X}^{\prime} \mathrm{CX} \beta+\beta^{\prime} \mathrm{X}^{\prime} \mathrm{C}^{\prime} \varepsilon+\varepsilon^{\prime} \mathrm{CX} \beta+\varepsilon^{\prime} \mathrm{C} \varepsilon=\varepsilon^{\prime} \mathrm{C} \varepsilon
$$

Assim, queremos minimizar a diferença entre (5.32) e (5.30), ou seja queremos minimizar

$$
\varepsilon^{\prime} \mathrm{C} \varepsilon-d^{-1} \varepsilon^{\prime} \varepsilon=\varepsilon^{\prime}\left(\mathrm{C}-d^{-1} \mathrm{I}\right) \varepsilon
$$

sujeito às restrições $\mathrm{CX}=0$ e $\operatorname{tr}(\mathrm{C})=1$.

Como $\varepsilon$ é variável aleatória, queremos obter

$$
\min \left\{\left\|\mathrm{C}-d^{-1} \mathbf{I}\right\|: \quad \mathrm{CX}=0, \quad \operatorname{tr}(\mathbf{C})=1\right\} .
$$

Quando a norma escolhida é a norma Euclideana, esses cálculos levam ao estimador de mínimos quadrados usual, dado por

$$
\hat{\sigma}^{2}=(n-p(\mathbf{X}))^{-1}\|\mathbf{y}-\mathbf{X} \hat{\boldsymbol{\beta}}\|^{2}
$$

onde $p(\mathrm{X})=\operatorname{posto}(\mathrm{X})$ e $\hat{\beta}$ é o estimador de mínimos quadrados de $\beta$.

Se relaxarmos a restrição de ser não-viesado, $\operatorname{tr}(\mathrm{C})=1$, chegaremos a

$$
\hat{\sigma}^{2}=d^{-1}\|\mathrm{y}-\mathrm{X} \hat{\boldsymbol{\beta}}\|^{2}
$$


que é o estimador de máxima verossimilhança de $\sigma^{2}$ sob a suposição de normalidade dos erros.

Se relaxarmos a condição $\mathrm{CX}=0$, a diferença que queremos minimizar é

$$
\left(\varepsilon^{\prime} \beta^{\prime}\right)\left(\begin{array}{c|c}
\mathrm{C}-d^{-1} \mathrm{I} & \mathrm{CX} \\
\hline \mathrm{X}^{\prime} \mathrm{C} & \mathrm{X}^{\prime} \mathrm{CX}
\end{array}\right)\left(\begin{array}{c}
\varepsilon \\
\beta
\end{array}\right) .
$$

Consideremos agora o modelo de efeitos mistos, com independência de erros e efeitos, isto é,

$$
\mathrm{y}=\mathrm{X} \alpha+\mathrm{Zb}+\mathrm{e}
$$

com

$$
\begin{array}{ll}
\mathbb{E}(\mathrm{b})=0 & \mathbb{E}(\mathbf{e})=0 \\
\operatorname{Var}(\mathrm{b})=S^{2} \mathrm{I} & \operatorname{Var}(\mathbf{e})=\sigma^{2} \mathbf{I} \\
\operatorname{Cov}(\mathrm{b}, \mathrm{e})=0 . &
\end{array}
$$

Seja $\varepsilon^{\prime}=\left(b^{\prime} e^{\prime}\right)$.

O estimador natural de uma combinação linear de $S^{2}$ e $\sigma^{2}, p_{1} S^{2}+p_{2} \sigma^{2}$, é

$$
p_{1}\left(d^{-1} \mathbf{b}^{\prime} \mathbf{b}\right)+p_{2}\left(d^{-1} \mathrm{e}^{\prime} \mathrm{e}\right)=\varepsilon^{\prime} d^{-1}\left(\begin{array}{cc}
p_{1} & 0 \\
0 & p_{2}
\end{array}\right) \varepsilon=\varepsilon^{\prime} \mathrm{D} \varepsilon
$$

Considerando o estimador quadrático $\mathrm{y}^{\prime} \mathrm{Cy}$, temos que

$$
\begin{aligned}
\mathrm{y}^{\prime} \mathrm{Cy} & =(\mathrm{X} \alpha+\mathrm{Zb}+\mathrm{e})^{\prime} \mathrm{C}(\mathrm{X} \alpha+\mathrm{Zb}+\mathrm{e}) \\
& =\alpha^{\prime} \mathrm{X}^{\prime} \mathrm{CX} \alpha+\alpha^{\prime} \mathrm{X}^{\prime} \mathrm{CZ} b+\alpha^{\prime} \mathrm{X}^{\prime} \mathrm{Ce}+\mathrm{b}^{\prime} \mathrm{Z}^{\prime} \mathrm{CX} \alpha \\
& +\mathrm{b}^{\prime} \mathrm{Z}^{\prime} \mathrm{CZb}+\mathrm{b}^{\prime} \mathrm{Z}^{\prime} \mathrm{Ce}+\mathrm{e}^{\prime} \mathrm{CX} \alpha+\mathrm{e}^{\prime} \mathrm{CZb}+\mathrm{e}^{\prime} \mathrm{Ce}
\end{aligned}
$$




$$
=\left(\begin{array}{lll}
\mathrm{b}^{\prime} & \mathrm{e}^{\prime} & \alpha^{\prime}
\end{array}\right)\left(\begin{array}{ccc}
\mathrm{Z}^{\prime} \mathrm{CZ} & \mathrm{Z}^{\prime} \mathrm{C} & \mathrm{Z}^{\prime} \mathrm{CX} \\
\mathrm{CZ} & \mathrm{C} & \mathrm{CX} \\
\mathrm{X}^{\prime} \mathrm{CZ} & \mathrm{X}^{\prime} \mathrm{C} & \mathrm{X}^{\prime} \mathrm{C}
\end{array}\right)\left(\begin{array}{l}
\mathrm{b} \\
\mathrm{e} \\
\alpha
\end{array}\right),
$$

que pode ser escrito como

$$
\left(\begin{array}{ll}
\varepsilon^{\prime} & \alpha^{\prime}
\end{array}\right)\left(\begin{array}{cc}
\mathrm{U}^{\prime} \mathrm{CU} & \mathrm{U}^{\prime} \mathrm{CX} \\
\mathrm{X}^{\prime} \mathrm{CU} & \mathrm{X}^{\prime} \mathrm{CX}
\end{array}\right)\left(\begin{array}{l}
\varepsilon \\
\alpha
\end{array}\right)
$$

onde $\mathrm{U}=(\mathrm{Z} \mathrm{I})$.

Queremos então minimizar a diferença $\mathrm{y}^{\prime} \mathrm{Cy}-\varepsilon^{\prime} \mathrm{D} \varepsilon$, que leva ao problema de minimizar

$$
\left\|\begin{array}{ll}
\mathrm{U}^{\prime} \mathrm{CU} & \mathrm{U}^{\prime} \mathrm{CX} \\
\mathrm{X}^{\prime} \mathrm{CU} & \mathrm{X}^{\prime} \mathrm{CX}
\end{array}\right\|
$$

Essa mesma idéia foi estendida para o modelo linear geral misto (5.36) abaixo.

$$
\mathrm{y}=\mathrm{X} \alpha+\mathrm{Zb}+\mathrm{e}
$$

$\operatorname{com} \operatorname{Var}(\mathrm{y})=\mathrm{V}_{1} S^{2}+\mathrm{V}_{2} \sigma^{2}$.

Seja

$$
\begin{aligned}
& \mathrm{V}=\mathrm{V}_{1}+\mathrm{V}_{2} \\
& \mathrm{R}=\mathrm{V}^{-1}\left(\mathrm{I}-\mathrm{X}\left(\mathrm{X}^{\prime} \mathrm{V}^{-1} \mathrm{X}\right)^{-1} \mathrm{X}^{\prime} \mathrm{V}^{-1}\right. \\
& \mathrm{Q}_{1}=\mathrm{y}^{\prime} \mathrm{RV} \mathrm{V}_{1} \mathrm{Ry} \\
& \mathrm{Q}_{2}=\mathrm{y}^{\prime} \mathrm{RV}_{2} \mathrm{Ry} .
\end{aligned}
$$

Usando a norma Euclideana, Rao (1971 a) mostrou que o MINQUE para a combinação linear de $S^{2}$ e $\sigma^{2}, p_{1} S^{2}+p_{2} \sigma^{2}$, é dado por

$$
\lambda_{1} Q_{1}+\lambda_{2} Q_{2}
$$


onde $\lambda_{1}$ e $\lambda_{2}$ são soluções do sistema

$$
\begin{aligned}
& \lambda_{1} \operatorname{tr}\left(\mathrm{RV}_{1} \mathrm{RV}_{1}\right)+\lambda_{2} \operatorname{tr}\left(\mathrm{RV}_{2} \mathrm{RV}_{1}\right)=p_{1} \\
& \lambda_{1} \operatorname{tr}\left(\mathrm{RV}_{1} \mathrm{RV}_{2}\right)+\lambda_{2} \operatorname{tr}\left(\mathrm{RV}_{2} \mathrm{RV}_{2}\right)=p_{2} .
\end{aligned}
$$

O modelo que estamos considerando, dado por (3.1), é um caso particular deste com

$$
\begin{aligned}
& \mathbf{V}_{1}=\left(\mathrm{I}_{n} \otimes \mathbf{J}_{T}\right)-\frac{1}{N} \mathbf{J}_{n T} \\
& \mathbf{V}_{2}=\mathrm{I}_{n T}, \\
& \mathbf{R}=\mathrm{I}_{n T}-\frac{1}{n}\left(\mathbf{J}_{n} \otimes \mathbf{I}_{T}\right)-\frac{1}{T+1}\left(\mathrm{I}_{n} \otimes \mathbf{J}_{T}\right)+\frac{1}{(T+1) n}\left(\mathbf{J}_{n} \otimes \mathbf{J}_{T}\right) \\
& \mathbf{Q}_{1}=\frac{T B}{(T+1)^{2}} \quad \mathrm{e} \quad \mathbf{Q}_{2}=A-\frac{(T+2) T}{(T+1)^{2}} B .
\end{aligned}
$$

$A$ e $B$ dados por (5.12) e (5.13), respectivamente.

O sistema composto pelas equações (5.39) e (5.40) passa, então, a ser

$$
\lambda_{1} \frac{T^{2}(n-1)}{(T+1)^{2}}+\lambda_{2} \frac{T(n-1)}{(T+1)^{2}}=p_{1}
$$




$$
\lambda_{2} \frac{T(n-1)}{(T+1)^{2}}+\lambda_{2} \frac{T(n-1)\left(T^{2}+T-1\right)}{(T+1)^{2}}=p_{2} .
$$

Para estimar $\sigma^{2}$ e $S^{2}$ basta tomar $p_{1}=0, p_{2}=1$ e $p_{1}=1, p_{2}=0$, respectivamente, e teremos

$$
\hat{\sigma}_{M}^{2}=\frac{A-B}{(n-1)(T-1)} \quad \text { e } \quad \hat{S}_{M}^{2}=\frac{T B-A}{T(n-1)(T-1)}
$$

ou seja, para o modelo que adotamos, os estimadores da teoria MINQUE, para as componentes de variância, usando norma Euclideana, são os mesmos estimadores obtidos pelo método da ANOVA.

\subsubsection{VARIÂNCIA DOS ESTIMADORES}

Os resultados mostrados até aqui indicam que, para o modelo adotado, o estimador MINQUE das componentes de variância, sob norma Euclideana, é o mesmo estimador da ANOVA e este, quando truncado no ponto zero, é o mesmo que o REML sob normalidade de efeitos e erros.

Por essa razão e pelo fato de ser o mais comumente utilizado, optamos por adotar o estimador da ANOVA, truncado no zero, que é dado por:

$$
\begin{gathered}
\hat{\sigma}^{2}=\hat{\sigma}_{A}^{2}=\frac{A-B}{(n-1)(T-1)} \\
\hat{S}^{2}= \begin{cases}\hat{S}_{A}^{2}=\frac{T B-A}{T(n-1)(T-1)} & \text { se } \hat{S}_{A}^{2} \geq 0 \\
0 & \text { se } \hat{S}_{A}^{2}<0\end{cases}
\end{gathered}
$$




$$
\begin{aligned}
\text { onde } & A=\sum_{i=1}^{n} \sum_{t=1}^{T}\left(y_{t i}-\bar{y}_{t .}\right)^{2} \\
\text { e } & B=\sum_{i=1}^{n} \sum_{t=1}^{T}\left(\bar{y}_{i}-\bar{y}_{. .}\right)^{2} .
\end{aligned}
$$

Os estimadores da ANOVA, $\hat{\sigma}_{A}^{2}$ e $\hat{S}_{A}^{2}$, são não-viesados para $\sigma^{2}$ e $S^{2}$ respectivamente e, portanto, $\hat{\sigma}^{2}$ é não-viesado para $\sigma^{2}$, mas $\hat{S}^{2}$ é viesado e, em média, superestima $S^{2}$.

As variâncias e covariância dos estimadores, cujas deduções encontram-se no apêndice D.3, foram obtidas com o uso das estatísticas $k$, introduzidas por Wishart ( 1952) e Tukey (1956a, 1956b, 1957a, 1957b). Os resultados obtidos foram:

$$
\begin{gathered}
\operatorname{Var}\left(\hat{\sigma}_{A}^{2}\right)=\frac{2}{(n-1)(T-1)} \sigma^{4} \\
\operatorname{Var}\left(\hat{S}_{A}^{2}\right)=\frac{(N-n)[n(N-1)-(N+1)]}{n(n-1)(N-2)(N-3)} S^{4} \\
+\frac{(N-n)\left(-N^{2} n+3 N^{2}-6 N+3 n+3\right)}{N n(n-1)(N-2)(N-3)}\left(S^{2}\right)^{2} \\
+\frac{4}{T(n-1)} S^{2} \sigma^{2}+\frac{2}{T(n-1)(T-1)} \sigma^{4} \\
\operatorname{Cov}\left(\hat{\sigma}_{A}^{2}, \hat{S}_{A}^{2}\right)=-\frac{2}{T(n-1)(T-1)} \sigma^{4}
\end{gathered}
$$

onde $\mathbb{E}\left(e_{t i}^{2}\right)=\sigma^{2} \quad, \quad \mathbb{E}\left(e_{t i}^{4}\right)=3 \sigma^{4}$,

$$
S^{2}=\frac{1}{N-1} \sum_{i=1}^{n} b_{i}^{2} \quad, \quad S^{4}=\frac{1}{N-1} \sum_{i=1}^{n} b_{i}^{4}
$$


Lembramos que obtivemos os estimadores supondo que o conjunto de dados fosse completo. Para calcular as estimativas, poderíamos utilizar somente o conjunto de indivíduos com dados completos, ignorando os indivíduos com dados perdidos; o conjunto de instantes para os quais todos os $n$ indivíduos responderam, descartando os instantes com dados perdidos, ou ainda considerar um método recursivo do tipo do algoritmo EM, mas usando os estimadores da ANOVA no lugar do estimador de máxima verossimilhança. O estudo de métodos recursivos fazem parte de planos de pesquisa futuros.

A variância do efeito aleatório (expressão 5.47) é bastante complicada e é difícil analisála para saber qual a melhor maneira de utilizar os dados para estimar a variância. Já com relação à variância dos erros, podemos tecer alguns comentários.

Seja

$n_{c}$ : número de indivíduos da amostra para os quais os dados dos $T$ instantes estão presentes

$T_{c}$ : número de instantes para os quais todos os $n$ indivíduos responderam.

Se optarmos por utilizar os indivíduos completos, teremos

$$
\operatorname{Var}\left(\hat{\sigma}_{A}^{2}\right)=\frac{2}{\left(n_{c}-1\right)(T-1)} \sigma^{4} ;
$$

se escolhermos utilizar os $T_{c}$ instantes completos,

$$
\operatorname{Var}\left(\hat{\sigma}_{A}^{2}\right)=\frac{2}{(n-1)\left(T_{c}-1\right)} \sigma^{4} .
$$

Isto significa que se $\frac{n_{c}-1}{n-1}>\frac{T_{c}-1}{T-1}$, é melhor utilizar os $n_{c}$ indivíduos completos porque levará a uma menor variância do estimador. 


\subsection{CASO MULTIVARIADO}

O modelo para o caso multivariado pode ser escrito com os dados (y) na forma de vetor, como no capítulo 4, ou então com os dados em forma de matriz.

Seja $\mathrm{Y}$ a matriz de dados. $\mathrm{Y}$ é uma matriz de dimensão $(n T \times p)$, onde $p$ é o número de variáveis observadas.

$$
\mathbf{Y}=\left(\begin{array}{cccc}
y_{111} & y_{211} & \cdots & y_{p 11} \\
y_{121} & y_{221} & \cdots & y_{p 21} \\
\vdots & \vdots & \vdots & \vdots \\
y_{1 T 1} & y_{2 T 1} & \cdots & y_{p T 1} \\
\vdots & \vdots & \vdots & \vdots \\
y_{11 n} & y_{21 n} & \cdots & y_{p 1 n} \\
y_{12 n} & y_{22 n} & \cdots & y_{p 2 n} \\
\vdots & \vdots & \vdots & \vdots \\
y_{1 T n} & y_{2 T n} & \cdots & y_{p T n}
\end{array}\right)
$$

Cada coluna de $\mathrm{Y}$ corresponde a uma variável observada. O modelo será escrito como

$$
\mathrm{Y}=\mathrm{X} \alpha+\mathrm{Zb}+\mathrm{e}
$$

onde $\alpha$ e b são matrizes de dimensão $(T \times p)$ e $(n \times p)$, respectivamente, e

$$
\mathrm{X}=\mathbf{1}_{n} \otimes \mathrm{I}_{T} \quad, \quad \mathrm{Z}=\mathrm{I}_{n} \otimes \mathbf{1}_{T}
$$

Seja $\mathbf{e}_{t i}^{\prime}=\left(e_{1 t i}, e_{2 t i}, \ldots, e_{p t i}\right)$. Então

$$
\operatorname{Var}\left(\mathbf{e}_{t i}^{\prime}\right)=\Sigma=\left(\begin{array}{cccc}
\sigma_{11} & \sigma_{12} & \cdots & \sigma_{1 p} \\
\sigma_{21} & \sigma_{22} & \cdots & \sigma_{2 p} \\
\vdots & \vdots & \vdots & \vdots \\
\sigma_{p 1} & \sigma_{p 2} & \cdots & \sigma_{p p}
\end{array}\right), \quad \text { para } t=1,2, \ldots, T
$$


Seja $\quad S_{j j^{\prime}}=\frac{1}{N-1} \sum_{i=1}^{n} b_{j i} b_{j^{\prime} i} \quad$ para quaisquer $j, j^{\prime}=1,2, \ldots, p$

$\mathrm{e}$

$$
\mathbf{S}=\left(\begin{array}{cccc}
S_{11} & S_{12} & \cdots & S_{1 p} \\
S_{21} & S_{22} & \cdots & S_{2 p} \\
\vdots & \vdots & \vdots & \vdots \\
S_{p 1} & S_{p 2} & \cdots & S_{p p}
\end{array}\right)
$$

Os estimadores não-viesados das matrizes de covariâncias $\mathbf{S}$ e $\boldsymbol{\Sigma}$ são análogas aos estimadores do caso univariado.

Seja $\mathrm{A}=\mathrm{Y}^{\prime}\left(\mathrm{I}_{n T}-\mathrm{X}\left(\mathrm{X}^{\prime} \mathrm{X}\right)^{-1} \mathrm{X}^{\prime}\right) \mathrm{Y}$.

No caso, $\mathrm{I}_{n T}-\mathrm{X}\left(\mathrm{X}^{\prime} \mathrm{X}\right)^{-1} \mathrm{X}^{\prime}=\left(\mathrm{I}_{n} \otimes \mathrm{I}_{T}\right)-\frac{1}{n}\left(\mathrm{~J}_{n} \otimes \mathrm{I}_{T}\right)$.

A esperança dos elementos da diagonal de A já foram calculadas no caso univariado. Em geral, considere $\mathrm{y}_{j}$, a $j$-ésima coluna de $\mathrm{Y}, j=1,2, \ldots, p$. Usando o resultado A.2 do apêndice $A$,

$$
\begin{gathered}
\mathbb{E}\left[\mathrm{y}_{j}^{\prime}\left(\mathbf{I}_{n T}-\mathbf{X}\left(\mathbf{X}^{\prime} \mathbf{X}\right)^{-1} \mathbf{X}^{\prime}\right) \mathbf{y}_{j^{\prime}}\right]= \\
\operatorname{tr}\left[( ( \mathbf { I } _ { n } \otimes \mathbf { I } _ { T } ) - \frac { 1 } { n } ( \mathbf { J } _ { n } \otimes \mathbf { I } _ { T } ) ) \left(\sigma_{j j^{\prime}}\left(\mathbf{I}_{n} \otimes \mathbf{I}_{T}\right)\right.\right. \\
\left.\left.+S_{j j^{\prime}}\left(\mathbf{I}_{n} \otimes \mathbf{J}_{T}\right)-\frac{S_{j j^{\prime}}}{N}\left(\mathbf{J}_{n} \otimes \mathbf{J}_{T}\right)\right)\right]=T(n-1)\left(\sigma_{j j^{\prime}}+S_{j j^{\prime}}\right) .
\end{gathered}
$$

Assim,

$$
\mathbb{E}(\mathrm{A})=T(n-1)(\mathrm{S}+\boldsymbol{\Sigma})
$$

Considere agora, 


$$
\mathbf{B}=\frac{1}{T}\left[\mathbf{Y}^{\prime}\left(\left(\mathbf{I}_{n} \otimes \mathbf{J}_{T}\right)-\frac{1}{n}\left(\mathbf{J}_{n} \otimes \mathbf{J}_{T}\right)\right) \mathbf{Y}\right]
$$

A esperança dos elementos de $\mathbf{B}$ é cada por

$$
\mathbb{E}\left[\frac{1}{T}\left(\mathrm{y}_{j}^{\prime}\left(\left(\mathrm{I}_{n} \otimes \mathrm{J}_{T}\right)-\frac{1}{n}\left(\mathbf{J}_{n} \otimes \mathbf{J}_{T}\right)\right) \mathrm{y}_{j^{\prime}}\right)\right]=(n-1)\left(T S_{j j^{\prime}}+\sigma_{j j^{\prime}}\right)
$$

e portanto

$$
\mathbb{E}(\mathbf{B})=(n-1)(T \mathbf{S}+\mathbf{\Sigma})
$$

Assim, de (5.49) e (5.50), podemos obter estimadores não-viesados das matrizes de covariâncias, que são

$$
\hat{\Sigma}=\frac{1}{(n-1)(T-1)}(\mathrm{A}-\mathrm{B}) \quad \text { e } \quad \hat{\mathrm{S}}=\frac{1}{T(n-1)(T-1)}(T \mathbf{B}-\mathbf{A})
$$

Pode ocorrer, entretanto, que $\hat{S}$ não seja uma matriz positiva definida. Nesse caso, uma alternativa é utilizar um procedimento como o sugerido por Bock e Petersen (1975), que consiste no seguinte.

Sejam $\mathbf{M}_{y}$ e $\mathrm{M}_{e}$ os quadrados médios tais que $\mathbb{I E}\left(\mathrm{M}_{y}\right)=\mathrm{S}+\Sigma$ e $\mathbb{I E}\left(\mathrm{M}_{e}\right)=\Sigma$, ou seja,

$$
\mathrm{M}_{y}=\frac{1}{T(n-1)}(\mathrm{A}) \quad \text { e } \quad \mathrm{M}_{e}=\frac{1}{(n-1)(T-1)}(\mathrm{A}-\mathrm{B}) .
$$

Sejam $\lambda_{1}, \lambda_{2}, \ldots, \lambda_{p}$ as raízes de $\left|\mathbf{M}_{y}-\lambda \mathbf{M}_{e}\right|=0$.

Como $M_{e}$ é positiva definida, existe uma matriz não-singular $\mathbf{P}$ tal que $M_{e}=P^{\prime} P$. Assim, 


$$
\left|\mathbf{M}_{y}-\lambda \mathbf{M}_{e}\right|=\left|\mathbf{M}_{y}-\lambda \mathbf{P}^{\prime} \mathbf{P}\right|=\left|\left(\mathbf{P}^{-1}\right)^{\prime} \mathbf{M}_{y} \mathbf{P}^{-1}-\lambda \mathbf{I}_{p}\right|,
$$

ou seja, $\lambda_{k}, k=1,2, \ldots, p$ são os autovalores de $\left(\mathrm{P}^{-1}\right)^{\prime} \mathbf{M}_{y} \mathrm{P}^{-1}$ e então existe uma matriz $\mathrm{U}$ tal que

$$
\mathrm{U}^{\prime}\left(\mathrm{P}^{-1}\right)^{\prime} \mathrm{M}_{y} \mathrm{P}^{-1} \mathrm{U}=\Lambda
$$

$\mathrm{e}$

$$
\mathrm{U}^{\prime} \mathrm{U}=\mathrm{I}_{p},
$$

onde $\boldsymbol{\Lambda}=\operatorname{diag}\left(\lambda_{1}, \lambda_{2}, \ldots, \lambda_{p}\right)$ (veja Mardia (1979)).

Agora, seja $\mathrm{R}=\mathrm{P}^{-1} \mathrm{U}$. De (5.52) e (5.53), temos que

$$
\mathrm{R}^{\prime} \mathrm{M}_{y} \mathrm{R}=\Lambda
$$

$\mathrm{e}$

$$
\mathrm{R}^{\prime} \mathrm{M}_{e} \mathrm{R}=\mathrm{I}_{p}
$$

Assim,

$$
\hat{\mathrm{S}}=\mathrm{M}_{y}-\mathrm{M}_{e}=\left(\mathrm{R}^{-1}\right)^{\prime}\left(\Lambda-\mathrm{I}_{p}\right) \mathrm{R}^{-1} .
$$

Para garantir que $\hat{\mathbf{S}}$ seja pelo menos positiva semi-definida, basta substituir $\boldsymbol{\Lambda}$ por $\boldsymbol{\Lambda}^{*}$ onde

$$
\Lambda^{*}=\operatorname{diag}\left(\lambda_{1}^{*}, \lambda_{2}^{*}, \ldots, \lambda_{p}^{*}\right) \quad \text { e } \quad \lambda_{k}^{*}=\max \left(\lambda_{k}, 1\right)
$$


Desse modo, $\hat{\mathbf{S}}$ será dada por

$$
\hat{\mathrm{S}}=\left(\mathrm{U}^{-1}\right)^{\prime}\left(\Lambda^{*}-\mathrm{I}_{p}\right) \mathrm{U}^{-1}
$$

que é igual ao estimador original de S, ciado pela expressão (5.51), se este for uma matriz positiva semi-definida.

\subsection{UMA ILUSTRAÇÃO NUMÉRICA}

Como ilustração numérica deste capítulo, voltamos a utilizar os dados de horas trabalhadas na produção (tabela 3.1) e os de total de pessoal ocupado (tabela 4.1), do Levantamento de Conjuntura - Índices FIESP.

Para estimar as componentes de variância, usamos as 11 empresas com dados completos, isto é, ignoramos as empresas 8 e 13 .

As estimativas foram obtidas pela aplicação da expressão (5.51), ou seja,

$$
\hat{\Sigma}=\frac{1}{(n-1)(T-1)}(\mathrm{A}-\mathrm{B}) \quad \text { e } \quad \hat{\mathrm{S}}=\frac{1}{T(n-1)(T-1)}(T \mathrm{~B}-\mathrm{A}),
$$

onde

$$
\begin{aligned}
& \mathbf{A}=\mathbf{Y}^{\prime}\left(\mathrm{I}_{n T}-\mathrm{X}\left(\mathrm{X}^{\prime} \mathrm{X}\right)^{-1} \mathrm{X}^{\prime}\right) \mathrm{Y} \mathrm{e} \\
& \mathbf{B}=\frac{1}{T}\left[\mathbf{Y}^{\prime}\left(\left(\mathrm{I}_{n} \otimes \mathrm{J}_{T}\right)-\frac{1}{n}\left(\mathrm{~J}_{n} \otimes \mathrm{J}_{T}\right)\right) \mathrm{Y}\right] .
\end{aligned}
$$

Os resultados obtidos foram:

$$
\begin{gathered}
\hat{\Sigma}=\left(\begin{array}{rr}
134,611 & 36,378 \\
36,378 & 399,084
\end{array}\right) \\
\hat{\mathbf{S}}=\left(\begin{array}{rr}
9052,093 & 68.116,493 \\
68.116,493 & 539.133,254
\end{array}\right) .
\end{gathered}
$$




\section{CAPÍTULO 6}

\section{ILUSTRAÇÕES NUMÉRICAS}

\subsection{INTRODUÇÃO}

Este capítulo tem como objetivo apresentar algumas ilustrações numéricas dos resultados obtidos nos capítulos anteriores.

Estamos apresentando três exemplos, onde os previsores das observações perdidas foram obtidos pela aplicação do programa computacional Mathematica (Wolfram (1991)).

\subsection{EXEMPLO 1: TOTAL DE PESSOAL OCUPADO E HORAS TRABALHADAS NA PRODUÇÃO}

Este exemplo já foi tratado no capítulo 3, na forma univariada, considerando somente 
horas trabalhadas na produção, e no capíutlo 4 , na forma bivariada, considerando as duas variáveis. Neste capítulo estamos apresentando os resultados encontrados pela aplicação do previsor que estamos propondo e de mais outros dois.

As tabelas 6.1 e 6.2 mostram os resultados obtidos para horas trabalhadas na produção e total de pessoal ocupado, respectivamente. Elas mostram os dados reais da empresa 13 $\left(y_{t i}\right)$, que foram recuperados posteriormente à data de fechamento do levantamento, os resultados do BLUP, caso univariado $\left(\hat{y}_{t i}(u n i)\right)$, os resultados do BLUP, caso multivariado $\left(\hat{y}_{t i}(\right.$ multi $\left.)\right)$ e outros dois previsores: a média das observações presentes no instante cujo dado está perdido $\left(\bar{y}_{t}\right)$ e a média dos dados presentes do indivíduo cuja observação está sendo imputada $\left(\bar{y}_{, i}\right)$. Além disso, as tabelas mostram as estimativas dos erros quadráticos médios dos previsores.

TABELA 6.1: RESULTADOS

IND.PAPEL E PAPELÃO DO ESTADO DE SÃO PAULO

(Estrato: empresa com mais de 500 empregados)

HORAS TRABALHADAS NA PRODUÇÃO (em milhares)

\begin{tabular}{|l|r|r|r|r|r|r|}
\hline & \multicolumn{2}{|c|}{ EMPRESA 8 / JUL } & \multicolumn{2}{c|}{ EMPRESA 13 / ABR } & \multicolumn{2}{|c|}{ EMPRESA 13 / JUL } \\
\cline { 2 - 7 } & Previsão & EQM & Previsão & EQM & Previsão & EQM \\
\hline$y_{t i}$ & - & - & 83,2 & - & 86,2 & - \\
$\hat{y}_{t i}($ uni $)$ & 170,8 & 106 & 85,2 & 218 & 86,0 & 220 \\
$\hat{y}_{t i}($ multi $)$ & 165,7 & 110 & 83,7 & 210 & 84,1 & 211 \\
$\bar{y}_{t .}$ & 182,0 & 10.022 & 180,4 & 9.952 & 182,0 & 10.022 \\
$\bar{y}_{. i}$ & 169,6 & 229 & 83,8 & 267 & 83,8 & 297 \\
\hline
\end{tabular}

Como pode-se verificar em ambas as tabelas, a média no instante $t\left(\bar{y}_{t}\right)$ não foi um bom previsor porque os dados presentes são de empresas, em média maiores do que as empresas com dados perdidos, especialmente a empresa 13. Isso fez com que os previsores superestimassem os dados de interesse. 
TABELA 6.2: RESULTADOS

IND.PAPEL E PAPELÃO DO ESTADO DE SÃO PAULO

(Estrato: empresa com mais de 500 empregados)

TOTAL DE PESSOAL OCUPADO

\begin{tabular}{|l|r|r|r|r|r|r|}
\hline & \multicolumn{2}{|c|}{ EMPRESA 8 JUL } & \multicolumn{2}{c|}{ EMPRESA 13 / ABR } & \multicolumn{2}{c|}{ EMPRESA 13 / JUL } \\
\cline { 2 - 7 } & Previsão & EQM & Previsão & EQM & Previsão & EQM \\
\hline$y_{t i}$ & - & - & 550 & - & 539 & - \\
$\hat{y}_{t i}$ (uni $)$ & 836 & 314 & 557 & 649 & 526 & 653 \\
$\hat{y}_{t i}($ multi $)$ & 837 & 314 & 557 & 649 & 527 & 653 \\
$\bar{y}_{t .}$ & 1378 & 588.581 & 1364 & 584.493 & 1378 & 588.581 \\
$\bar{y}_{. i}$ & 860 & 4.517 & 546 & 5.939 & 546 & 8.252 \\
\hline
\end{tabular}

Com relação à média do indivíduo $i\left(\bar{y}_{. i}\right)$, os resultados foram melhores, pois essas variáveis têm variação pequena ao longo do tempo. Entretanto, sob o modelo adotado, esse previsor é viesado.

O BLUP $\left(\hat{y}_{t i}\right)$ apresentou bons resultados para ambas as variáveis pois esse previsor, de certa forma, corrige a característica acima, da empresa 13 ser menor do que a média do grupo.

O fato de usar as duas variáveis conjuntamente para a previsão $\left(\hat{y}_{t i}(\right.$ multi $\left.)\right)$, trouxe melhora no resultado da empresa 13 , no mês de abril, para a variável horas trabalhadas na produção. No mês de julho o resultado piorou.

Para a variável total de pessoal ocupado, usar as duas variáveis conjuntamente nada acrescentou ao resultado univariado.

O BLUP apresentou resultados bem superiores aos da média no instante $t$ e aos da média do indivíduo $i$, uma vez que o ganho em termos de EQM foi bastante expressivo. Esse alto ganho é devido ao fato que $K$, a razão entre as componentes da variância, é grande. Conforme já concluído no capítulo 3, quanto maior o valor de $K$, maior o ganho em se usar o BLUP no lugar da média no instante $t$. O ganho em termos de EQM para 
a variável total de pessoal ocupado foi bem maior do que o ganho para a variável horas trabalhadas na produção, pois o valor de $K$ para pessoal é bem maior do que o valor de $K$ para horas: $\hat{K}_{P}^{\prime}=1.350,9$ enquanto que $\hat{K}_{H}=67,2$.

\subsection{EXEMPLO 2: TOTAL DE SALÁRIOS NOMINAIS}

Outros dados da pesquisa FIESP que consideramos são os de total de salários nominais, para o período de janeiro a abril de 1994. A tabela 6.3 abaixo mostra os dados, com a ausência da observação da empresa 13 no mês de abril, que foi recuperada posteriormente.

TABELA 6.3: DADOS

IND.PAPEL E PAPELÃO DO ESTADO DE SÃO PAULO

(Estrato: empresa com mais de 500 empregados)

TOTAL DE SALÁRIOS NOMINAIS (em milhões)

\begin{tabular}{|l|r|r|r|r|}
\hline & \multicolumn{5}{|c|}{ MESES/94 } \\
\cline { 2 - 5 } & JAN & FEV & MAR & ABR \\
\hline EMPRESA 1 & 1.072 & 1.661 & 1.797 & 1.837 \\
EMPRESA 2 & 667 & 1.250 & 1.482 & 1.987 \\
EMPRESA 3 & 687 & 1.113 & 1.919 & 2.283 \\
EMPRESA 4 & 442 & 765 & 1.238 & 1.432 \\
EMPRESA 5 & 444 & 1.041 & 1.132 & 1.838 \\
EMPRESA 6 & 351 & 575 & 752 & 1.099 \\
EMPRESA 7 & 334 & 497 & 821 & 1.113 \\
EMPRESA 8 & 131 & 237 & 392 & 438 \\
EMPRESA 9 & 338 & 649 & 709 & 866 \\
EMPRESA 10 & 127 & 236 & 292 & 414 \\
EMPRESA 11 & 233 & 345 & 670 & 693 \\
EMPRESA 12 & 87 & 137 & 241 & 391 \\
EMPRESA 13 & 157 & 242 & 426 & \\
\hline
\end{tabular}

Fonte: FIESP

Os resultados encontrados foram: 


$$
\begin{array}{cl}
\hat{\sigma}_{S}^{2}=44.645,971 \quad \hat{S}_{S}^{2}=221.997,054 \quad K=\frac{\hat{S}_{S}^{2}}{\hat{\sigma}_{S}^{2}}=4,972 \\
y_{T n}=669 & \\
\hat{y}_{T n}=810 & \left(E \hat{Q} M\left(\hat{y}_{T n}\right)\right)=63.476 \\
\bar{y}_{T .}=1.199 & \left(E \hat{Q} M\left(\bar{y}_{T .}\right)\right)=288.845 \\
\bar{y}_{. n}=275 & \left(E \hat{Q} M\left(\bar{y}_{. n}\right)\right)=351.731
\end{array}
$$

Neste caso, a estimativa obtida através do BLUP $\left(\hat{y}_{T_{n}}=810\right)$ foi melhor do que as estimativas obtidas pela média em abril $\left(\bar{y}_{T} .=1.199\right)$ e pela média da empresa 13 $\left(\bar{y}_{n}=275\right)$. Isto se deve ao fato de que, por um lado, a empresa 13 é uma das que tem os menores valores de salários da amostra e por isso a média no instante $T$ superestima a observação perdida. Por outro lado, essa variável tem tendência crescente no tempo e então a média do indivíduo acaba por subestimar a observação ausente, além do que é viesada, pois, neste caso, nitidamente existe efeito do tempo.

\subsection{EXEMPLO 3: OS DADOS DE POTTHOFF e ROY}

O último exemplo que consideramos foi a aplicação de nossos resultados aos dados bastante conhecidos, apresentados por Potthoff e Roy (1964).

Esse experimento foi realizado na Escola de Odontologia da Universidade da Carolina do Norte e, para 11 meninas e 16 meninos, foram medidas a distância, em milímetros, do centro da pituitária até a fissura pterio-maxilar. Essa distância foi medida em quatro idades, 8, 10, 12 e 14 anos. Os dados estão apresentados na tabela 6.4 a seguir. 
TABELA 6.4: DADOS

DISTÂNCIA ENTRE O CENTRO DA PITUITÁRIA

E FISSURA PTERIO-MAXILAR (em milímetros)

\begin{tabular}{|l|l|l|l|l|}
\hline \multirow{2}{*}{ INDIVÍDUO } & \multicolumn{4}{|c|}{ TEMPO } \\
\cline { 2 - 5 } & 8 & 10 & 12 & 14 \\
\hline meninos & & & & \\
1 & 21 & 20 & 21,5 & 23 \\
2 & 21 & 21,5 & 24 & 25,5 \\
3 & 20,5 & 24 & 24,5 & 26 \\
4 & 23,5 & 24,5 & 25 & 26,5 \\
5 & 21,5 & 23 & 22,5 & 23,5 \\
6 & 20 & 21 & 21 & 22,5 \\
7 & 21,5 & 22,5 & 23 & 25 \\
8 & 23 & 23 & 23,5 & 24 \\
9 & 20 & 21 & 22 & 21,5 \\
10 & 16,5 & 19 & 19 & 19,5 \\
11 & 24,5 & 25 & 28 & 28 \\
meninas & & & & \\
12 & 26 & 25 & 29 & 31 \\
13 & 21,5 & 22,5 & 23 & 26,5 \\
14 & 23 & 22,5 & 24 & 27,5 \\
15 & 25,5 & 27,5 & 26,5 & 27 \\
16 & 20 & 23,5 & 22,5 & 26 \\
17 & 24,5 & 25,5 & 27 & 28,5 \\
18 & 22 & 22 & 24,5 & 26,5 \\
19 & 24 & 21,5 & 24,5 & 25,5 \\
20 & 23 & 20,5 & 31 & 26 \\
21 & 27,5 & 28 & 31 & 31,5 \\
22 & 23 & 23 & 23,5 & 25 \\
23 & 21,5 & 23,5 & 24 & 28 \\
24 & 17 & 24,5 & 26 & 29,5 \\
25 & 22,5 & 25,5 & 25,5 & 26 \\
26 & 23 & 24,5 & 26 & 30 \\
27 & 22 & 21,5 & 23,5 & 25 \\
\hline
\end{tabular}

Fonte: University of North Carolina Dental School

Rao (1987) também considerou esse conjunto de dados. Seu interesse era prever a observação no último instante (aos 14 anos), com base nas observações dos 3 instantes 
anteriores. Os métodos de imputação aplicados, cujos detalhes podem ser encontrados em Rao (1987), foram:

- Previsão linear

- Regressão linear

- Regressão inversa

- Modelos de curvas de crescimento

- Regressão individual

- Regressão sobre coeficientes polinomiais

- Calibração

- Previsão de Bayes empírica.

Além disso, ele fez as previsões considerando todo o conjunto de dados ou só parte dele. Primeiro ele utilizou os 4 instantes, depois os 3 últimos $(10,12$ e 14 anos) e por fim só os 2 últimos (12 e 14 anos).

Para escolher qual dos métodos de imputação é o melhor, ele usou a técnica LOO (leave-one-out), descrita em Lachenbruch (1975), que consiste em escolher o método cuja estimativa do erro quadrático médio é a menor. No caso, a técnica do LOO consiste no seguinte:

a) para cada indivíduo $i, i=1,2, \ldots, n$, preve-se $y_{T i}$ usando todo o conjunto de observações mas omitindo a informação $y_{T i}$; 
b) como $y_{T i}, i=1,2, \ldots, n$ é na verdade conhecido, calcula-se

$$
E \hat{Q} M\left(\hat{y}_{T i}\right)=\frac{1}{n} \sum_{i=1}^{n}\left(\hat{y}_{T_{i}}-y_{T_{i}}\right)^{2}
$$

c) o método de previsão escolhido é aquele que tem o menor $E \hat{Q} M\left(\hat{y}_{T_{i}}\right)$.

Aplicamos o BLUP aos dados da tabela 6.4 considerando, como Rao, todas as informações, só os três últimos e só os dois últimos instantes. A tabela 6.5 abaixo, mostra as estimativas do erro quadrático médio para os métodos propostos por Rao e pelo método que estamos propondo.

TABELA 6.5: RESULTADOS

ERRO QUADRÁTICO MÉDIO ESTIMADO

\begin{tabular}{|l|r|r|r|}
\hline MÉTODO & $t_{1}$ a $t_{4}$ & $t_{2}$ a $t_{4}$ & $t_{3}$ e $t_{4}$ \\
\hline Regressão direta & 4,430 & 3,588 & 3,665 \\
Regressão inversa & 6,211 & 5,227 & 4,929 \\
Regressão individual (grau 2) & 47,398 & - & - \\
Regressão individual (grau 1) & 3,998 & 12,426 & - \\
Regr.s/ coef. polinomiais (grau 2) & 4,430 & - & - \\
Regr.s/ coef. polinomiais (grau 1) & 3,288 & 3,585 & - \\
Calibração (grau 2) & 9,483 & - & - \\
Calibração (grau 1) & 3,680 & 8,358 & - \\
Bayes empírica (grau 1) & 2,322 & - & - \\
BLUP & 2,777 & 2,321 & 2,928 \\
\hline
\end{tabular}

A tabela mostra que, considerando todo o conjunto de dados $\left(\begin{array}{lll}t_{1} & \text { a } & t_{4}\end{array}\right)$, o nosso método só foi inferior a um dos métodos propostos por Rao (previsão de Bayes empírica). Já levando em conta todas as combinações consideradas, o método que estamos propondo, aplicado aos três últimos instantes, é tão bom quanto este. No caso, a estimativa do erro quadrático médio foi de 2,321 . 


\section{CONCLUSÕES}

O objetivo inicial deste trabalho era encontrar o "melhor" previsor das observações perdidas, no caso de termos como amostra um painel sorteado de uma população finita. O conceito de melhor, utilizado neste contexto, foi o de previsor linear, não-viesado, de erro quadrático médio mínimo. Esse previsor é conhecido na literatura como BLUP (Best Linear Unbiased Predictor).

Estávamos interessados em um modelo simples (descrito nos capítulos 3 e 4), que traz limitações ao impor uma estrutura de covariâncias constante no tempo, mas acabamos por obter os resultados para um modelo mais geral, que engloba modelos com uma estrutura de covariância mais ampla e também modelos de curva de crescimento. Esse modelo foi descrito no capítulo 2 e é o seguinte:

$$
\mathrm{y}=\mathrm{X} \alpha+\mathrm{Zb}+\mathrm{e}
$$

cujo previsor para as observações ausentes é 


$$
\hat{\mathrm{y}}_{a}=\left(\mathrm{I}-\mathrm{E}^{\prime} \mathrm{QE}\right)^{-1} \mathrm{E}^{\prime} \mathrm{QFF}^{\prime} \mathrm{y} .
$$

Obtivemos também os estimadores dos efeitos fixos e os previsores dos efeitos aleatórios desse modelo (BLUP's), que nada mais são do que os previsores para o caso com observações completas, aplicado ao vetor de observações y, completado pelo previsor dos dados ausentes. No caso dos efeitos fixos, é o estimador de mínimos quadrados generalizado.

Um dos fatos mais interessantes é que os resultados que obtivemos para o modelo mais simples, são interpretáveis e de fácil aplicação, o que os transforma em resultados de grande utilidade.

Outro fato interessante é a conexão que fizemos com os resultados válidos para o modelo usualmente utilizado para populações infinitas, onde os $b_{i}$ 's provém de uma distribuição normal e são considerados independentes. Concluímos que os previsores para as observações ausentes são os mesmos, a menos de uma correção no caso de população finita de tamanho $N$, dada por $\frac{N}{N-1}$. Como adotamos que $\operatorname{Var}\left(b_{i}\right)=\frac{N-1}{N} S^{2}$, temos que para populações infinitas $K=\frac{\operatorname{Var}\left(b_{i}\right)}{\operatorname{Var}\left(e_{t i}\right)}$ enquanto que para populações finitas $K=\frac{N}{N-1} \frac{\operatorname{Var}\left(b_{i}\right)}{\operatorname{Var}\left(e_{t i}\right)}$. Quando o tamanho da população cresce, os previsores se aproximam.

É claro que há muito por ser feito nessa linha de pesquisa. A continuidade deste trabalho consistirá em:

- estudar modelos que permitam considerar outro tipo de estrutura de correlação no tempo, como por exemplo a correlação serial;

- estudar com detalhe outras estruturas para o modelo de previsão, como por exemplo modelos de curvas de crescimento; 
- estudar outros métodos para estimar as variâncias e covariâncias em populações finitas com dados incompletos, incluindo métodos iterativos;

- estudar, no caso multivariado, a falta parcial do questionário - a não-resposta de item;

- estudar o método de estimação de máxima verossimilhança, inclusive a adaptação do algoritmo EM.

Com relação ao último tópico dessa pesquisa futura, e a outros que necessitem da distribuição conjunta de efeitos e erros, já demos um passo, uma vez que obtivemos a função distribuição de probabilidade dos dados completos para o modelo misto aqui considerado.

Dado que os erros tem distribuição normal e são independentes $\left(\mathrm{e} \sim N\left(0, \sigma^{2} \mathrm{I}\right)\right)$, essa função é uma mistura de normais, dada por:

$$
\frac{(N-n)}{N}\left[\sum_{i_{1}=1}^{N} \prod_{t=1}^{T} \Phi\left(y_{t 1}-b_{i_{1}}\right)\left(\sum_{\substack{i_{2}=1 \\ i_{2} \neq i_{1}}}^{N} \prod_{t=1}^{T} \Phi\left(y_{t 2}-b_{i_{2}}\right) \ldots\left(\sum_{\substack{i_{n}=1 \\ i_{n} \neq i_{1}}}^{N} \prod_{t=1}^{T} \Phi\left(y_{t n}-b_{i_{n}}\right)\right)\right)\right]
$$

onde $\Phi$ é a função distribuição da distribuição normal com média zero e variância $\sigma^{2}$.

Acreditamos que resultados interessantes e de grande utilidade prática podem ser obtidos nessa área. 


\section{APÊNDICE A}

\section{RESULTADOS UTILIZADOS}

\section{A.1. PRODUTO DE KRONECKER}

Sejam $\mathbf{A}=\left(a_{i j}\right)$ e $\mathbf{B}=\left(b_{i j}\right)$ matrizes de dimensão $m \times n$ e $p \times q$, respectivamente. $\mathrm{O}$ produto de Kronecker, indicado por $\mathbf{A} \otimes \mathbf{B}$, é uma matriz de dimensão $m p \times n q$, definida por

$$
\mathrm{A} \otimes \mathbf{B}=\left(a_{i j} \mathrm{~B}\right)
$$

Propriedades: $\mathbf{A}, \mathbf{A}_{1}, \mathbf{A}_{2}, \mathbf{B}, \mathbf{B}_{1}, \mathbf{B}_{2}$ matrizes; $a, b$ escalares.

(a) $0 \otimes A=A \otimes 0=0$;

(b) $\left(\mathbf{A}_{1}+\mathbf{A}_{2}\right) \otimes B=\left(\mathbf{A}_{1} \otimes \mathbf{B}\right)+\left(\mathbf{A}_{2} \otimes \mathbf{B}\right)$;

(c) $\mathbf{A} \otimes\left(\mathrm{B}_{1}+\mathrm{B}_{2}\right)=\left(\mathrm{A} \otimes \mathrm{B}_{1}\right)+\left(\mathrm{A} \otimes \mathrm{B}_{2}\right)$; 
(d) $a \mathrm{~A} \otimes b \mathrm{~B}=a b \mathrm{~A} \otimes \mathbf{B}$

(e) $A_{1} A_{2} \otimes B_{1} B_{2}=\left(A_{1} \otimes B_{1}\right)\left(A_{2} \otimes B_{2}\right)$;

(f) $(A \otimes B)^{-1}=A^{-1} \otimes B^{-1}$, se as inversas existem;

$(\mathrm{g})(\mathbf{A} \otimes \mathbf{B})^{-}=\mathbf{A}^{-} \otimes \mathbf{B}^{-}$, para qualquer inversa generalizada;

(h) $(\mathrm{A} \otimes \mathbf{B})^{\prime}=\mathrm{A}^{\prime} \otimes \mathrm{B}^{\prime}$;

(i) $(\mathrm{A} \otimes \mathrm{B})\left(\mathrm{A}^{-1} \otimes \mathrm{B}^{-1}\right)=\mathrm{I}$.

\section{A.2. ESPERANÇA DE FORMAS QUADRÁTICAS}

Sejam $\mathrm{x}$ e $\mathrm{y}$ vetores e A matriz.

Seja $\mathbb{E}(\mathrm{x})=\mu, \operatorname{Var}(\mathrm{x})=\mathrm{C}_{1}, \mathbb{E}(\mathrm{y})=\nu, \operatorname{Var}(\mathrm{y})=\mathrm{C}_{2}, \mathbb{E}\left[(\mathrm{x}-\mu)(\mathrm{y}-\boldsymbol{\nu})^{\prime}\right]=\mathrm{C}_{12}$,

$\mathrm{C}_{21}=\mathrm{C}_{12}^{\prime}$. Então $\mathbb{E}\left(\mathrm{x}^{\prime} \mathrm{A} \mathrm{y}\right)=\operatorname{tr}\left(\mathrm{AC}_{21}\right)+\mu^{\prime} \mathrm{A} \boldsymbol{\nu}$

\section{A.3. INVERSA DE MATRIZES}

(1) Sejam $c$ e $b$ escalares e A uma matriz quadrada de dimensão $n$, dada por

$$
\begin{aligned}
& \mathbf{A}=\left(\begin{array}{cccc}
c+b & c & \cdots & c \\
c & c+b & \cdots & c \\
\vdots & \vdots & & \vdots \\
c & c & \cdots & c+b
\end{array}\right) \\
& \mathbf{A}=c \mathbf{J}_{n}+b \mathbf{I}_{n}
\end{aligned}
$$


A inversa de $\mathrm{A}$ é dada por

$$
\mathrm{A}^{-1}=\left(a_{i j}\right) \text { onde } a_{i j}= \begin{cases}\frac{1}{b}-\frac{c}{(n c+b) b} & , i=j \\ -\frac{c}{(n c+b) b} & , i \neq j\end{cases}
$$

ou seja

$$
\mathrm{A}^{-1}=\frac{1}{b} \mathrm{I}_{n}-\frac{c}{(n c+b) b} \mathrm{~J}_{n}
$$

(2) Este resultado é uma extensão do resultado acima, para o caso em que B e C são matrizes.

Sejam B e C duas matrizes quadradas de dimensão $T, \operatorname{com} \operatorname{posto}(\mathbf{B})=T$.

Seja A uma matriz quadrada de dimensão $n T$, dada por

$$
\mathrm{A}=\left(\begin{array}{cccc}
\mathrm{C}+\mathrm{B} & \mathrm{C} & \cdots & \mathrm{C} \\
\mathrm{C} & \mathrm{C}+\mathrm{B} & \cdots & \mathrm{C} \\
\vdots & \vdots & & \vdots \\
\mathrm{C} & \mathrm{C} & \cdots & \mathrm{C}+\mathrm{B}
\end{array}\right)
$$

ou seja, $\mathrm{A}=\left(\mathrm{I}_{n} \otimes \mathrm{B}\right)+\left(\mathrm{J}_{n} \otimes \mathrm{C}\right)$.

A inversa de $\mathbf{A}$ é dada por

$$
\mathrm{A}^{-1}=\left(\mathrm{I}_{n} \otimes \mathrm{B}^{-1}\right)-\mathrm{J}_{n} \otimes\left[(n \mathrm{C}+\mathrm{B})^{-1} \mathrm{CB}^{-1}\right] .
$$

\section{A.4. IGUALDADE DE MATRIZES}

Sejam A e D matrizes quadradas não singulares de dimensão $m$ e $n$, respectivamente. Seja B uma matriz de dimensão $m \times n$. Então,

$$
\begin{aligned}
\left(\mathbf{A}+\mathbf{B D B}^{\prime}\right)^{-1} & =\mathbf{A}^{-1}-\mathbf{A}^{-1} \mathbf{B}\left(\mathbf{B}^{\prime} A^{-1} \mathbf{B}+\mathbf{D}^{-1}\right)^{-1} \mathbf{B}^{\prime} A^{-1} \\
& =A^{-1}-A^{-1} B E B^{\prime} A^{-1}+A^{-1} B E(E+D)^{-1} \mathbf{E B}\left(A^{\prime}\right)^{-1}
\end{aligned}
$$

onde $E=\left(B^{\prime} A^{-1} B\right)^{-1}$ 


\section{A.5. DERIVADAS DE FUNÇÕES DE MATRIZES}

(1) Sejam A, U, V matrizes e a escalar.
(a) $\partial \mathbf{A}=0$
(b) $\partial(a \mathrm{U})=a \partial \mathrm{U}$;
(c) $\partial(\mathrm{U}+\mathrm{V})=\partial \mathrm{U}+\partial \mathrm{V}$
(d) $\partial(\mathrm{U}-\mathrm{V})=\partial \mathrm{U}-\partial \mathrm{V}$;
(e) $\partial(\mathrm{UV})=(\partial \mathrm{U}) \mathrm{V}+\mathrm{U}(\partial \mathrm{V})$;
(f) $\partial \mathbf{U}^{\prime}=(\partial \mathbf{U})^{\prime}$;
(g) $\partial v e c \mathrm{U}=v e c \partial \mathrm{U}$;
(h) $\partial \operatorname{tr} \mathrm{U}=\operatorname{tr} \partial \mathrm{U}$.

(2) Casos particulares:

A matriz simétrica, $\mathrm{B}, \mathrm{X}$, e $\mathrm{Y}$ vetores de dimensão adequada, $f(\mathrm{X})$ função escalar de $\mathbf{X}$.

\begin{tabular}{l|l}
$f(\mathrm{X})$ & $\partial f(\mathrm{X}) / \partial \mathrm{X}$ \\
\hline $\mathrm{B}^{\prime} \mathrm{X}$ & $\mathrm{B}$ \\
$\mathrm{X}^{\prime} \mathrm{AY}$ & $\mathrm{AY}$ \\
$\mathrm{X}^{\prime} \mathrm{X}$ & $2 \mathrm{X}$ \\
$\mathrm{X}^{\prime} \mathrm{AX}$ & $2 \mathrm{AX}$
\end{tabular}

se $\mathrm{X}$ é simétrica

\begin{tabular}{c|c}
$f(\mathbf{X})$ & $\partial f(\mathbf{X}) / \partial \mathbf{X}$ \\
\hline $\mathbf{Y}^{\prime} \mathbf{X B}$ & $\mathbf{Y B}^{\prime}$ \\
$\mathbf{Y}^{\prime} \mathbf{X Y}$ & $\mathbf{Y Y}^{\prime}$
\end{tabular}

A matriz simétrica, B, X, e Y matrizes de dimensão adequada, a vetor.

\begin{tabular}{l|l}
\multicolumn{1}{c|}{$f(\mathrm{X})$} & $\partial f(\mathrm{X}) / \partial \mathbf{X}$ \\
\hline $\mathbf{a}^{\prime} \mathbf{X} \mathbf{A X} \mathbf{X}^{\prime} \mathbf{a}$ & $2 \mathbf{a a}^{\prime} \mathbf{X} \mathbf{A}$ \\
$\mathbf{a}^{\prime} \mathbf{X A Y} \mathbf{a}$ & $\mathbf{a a}^{\prime} \mathbf{Y A}$ \\
$\operatorname{tr}(\mathbf{Y X})$ & $\mathbf{Y}^{\prime}$ \\
$\operatorname{tr}(\mathbf{Y X B})$ & $\mathbf{Y}^{\prime} \mathbf{B}^{\prime}$
\end{tabular}




\section{APÊNDICE B}

CASO UNIVARIADO

B.1. UMA OBSERVAÇÃO PERDIDA NO ÚLTIMO INSTANTE

$$
\widehat{y}_{T n}=\left\{I_{m}-E^{\prime} \mathrm{QE}\right\}^{-1} \mathrm{E}^{\prime} \mathrm{QFF} \mathrm{F}^{\prime}
$$

onde

$$
\begin{aligned}
& \mathrm{Q}=\mathrm{W}^{-1} \mathrm{X}\left(\mathrm{X}^{\prime} \mathrm{W}^{-1} \mathrm{X}\right)^{-1} \mathrm{X}^{\prime} \mathrm{W}^{-1}+\mathrm{I}_{n T}-\mathrm{W}^{-1} \\
& \mathrm{~W}^{-1}=\left(\mathrm{I}_{n} \otimes \mathrm{I}_{T}\right)-a\left(\mathrm{I}_{n} \otimes \mathrm{J}_{T}\right)+b\left(\mathrm{~J}_{n} \otimes \mathrm{J}_{T}\right) \\
& \left(\mathrm{X}^{\prime} \mathrm{W}^{-1} \mathrm{X}\right)^{-1}=\frac{1}{n}\left[\mathrm{I}_{T}-\frac{(n b-a)}{(T(n b-a)+1)} \mathrm{J}_{T}\right] \\
& a=\frac{K}{T K+1} \quad b=\frac{K}{N(T K+1)(T K(1-f)+1)} \quad K=\frac{S^{2}}{\sigma^{2}} \\
& \mathrm{X}=\mathbf{1}_{n} \otimes \mathrm{I}_{T} .
\end{aligned}
$$


Assim,

$$
\begin{aligned}
& \mathrm{W}^{-1} \mathrm{X}=\left(\mathbf{1}_{n} \otimes \mathrm{I}_{T}\right)+(n b-a)\left(\mathbf{1}_{n} \otimes \mathbf{J}_{T}\right) \\
& \mathrm{W}^{-1} \mathrm{X}\left(\mathrm{X}^{\prime} \mathrm{W}^{-1} \mathrm{X}\right)^{-1}=\frac{1}{n}\left(\mathbf{1}_{n} \otimes \mathrm{I}_{T}\right) \\
& \mathrm{W}^{-1} \mathrm{X}\left(\mathrm{X}^{\prime} \mathrm{W}^{-1} \mathrm{X}\right)^{-1} \mathrm{X}^{\prime} \mathrm{W}^{-1}=\frac{1}{n}\left(\mathrm{~J}_{n} \otimes \mathrm{I}_{T}\right)+\frac{(n b-a)}{n}\left(\mathrm{~J}_{n} \otimes \mathrm{J}_{T}\right) \\
& \mathrm{Q}=\frac{1}{n}\left(\mathrm{~J}_{n} \otimes \mathrm{I}_{T}\right)+a\left(\mathrm{I}_{n} \otimes \mathrm{J}_{T}\right)-\frac{a}{n}\left(\mathrm{~J}_{n} \otimes \mathrm{J}_{T}\right) .
\end{aligned}
$$

Tomando

$$
\underset{(n T \times 1)}{\mathrm{E}}=\left(\begin{array}{c}
0 \\
0 \\
\vdots \\
0 \\
1
\end{array}\right) \underset{(n T \times(n T-1))}{\mathbf{F}}=\left(\begin{array}{cccc}
1 & 0 & \cdots & 0 \\
0 & 1 & \cdots & 0 \\
\vdots & \vdots & & \vdots \\
0 & 0 & \cdots & 1 \\
0 & 0 & \cdots & 0
\end{array}\right)
$$

temos

$$
\begin{aligned}
& \mathbf{E}^{\prime} \mathrm{Q}=(\underbrace{0,0, \ldots, 0, \frac{1}{n}}, \underbrace{0,0, \ldots, 0, \frac{1}{n}}, \ldots, \underbrace{0,0, \ldots, 0, \frac{1}{n}}) \\
& +(\underbrace{0,0, \ldots, 0,0}, \underbrace{0,0, \ldots, 0,0}, \ldots, \underbrace{a, a, \ldots, a, a}) \\
& \mathbf{E}^{\prime} \mathbf{Q E}=\frac{a}{n}, \frac{a}{n}, \ldots, \frac{a}{n}, \frac{a}{n}, \underbrace{\frac{a}{n}, \frac{a}{n}, \ldots, \frac{a}{n}, \frac{a}{n}}, \ldots, \underbrace{\frac{a}{n}, \frac{a}{n}, \ldots, \frac{a}{n}, \frac{a}{n}}) \\
& \mathbf{I}_{m}-\mathbf{E}^{\prime} \mathrm{QE}=\frac{(n-1)(1-a)}{n}
\end{aligned}
$$




$$
\begin{aligned}
& \underset{(n T \times n T)}{\mathbf{F F}^{\prime}}=\left(\begin{array}{ccccc}
1 & 0 & \cdots & 0 & 0 \\
0 & 1 & \cdots & 0 & 0 \\
\vdots & \vdots & & \vdots & \vdots \\
0 & 0 & \cdots & 1 & 0 \\
0 & 0 & \cdots & 0 & 0
\end{array}\right) \\
& \mathrm{E}^{\prime} \mathrm{QFF} \mathrm{F}^{\prime}=(\underbrace{-\frac{a}{n},-\frac{a}{n}, \ldots, \frac{1-a}{n}}, \underbrace{-\frac{a}{n},-\frac{a}{n}, \ldots, \frac{1-a}{n}}, \ldots, \underbrace{\left.a-\frac{a}{n}, a-\frac{a}{n}, \ldots, 0\right)} .
\end{aligned}
$$

A combinação das $(n-1)$ observações no instante $T$ é

$$
\frac{n}{(n-1)(1-a)} \frac{(1-a)}{n} \sum_{i=1}^{n-1} y_{T i}=\frac{1}{n-1} \sum_{i=1}^{n-1} y_{T i}
$$

das $T-1$ observações do $n$-ésimo indivíduo é

$$
\frac{n}{(n-1)(1-a)} \frac{a(n-1)}{n} \sum_{t=1}^{T-1} y_{t n}=\frac{K}{(T-1) K+1} \sum_{t=1}^{T-1} y_{t n}
$$

e das $(n-1)(T-1)$ observações dos indivíduos 1 a $n-1$ nos instantes 1 a $T-1$

$$
-\frac{n}{(n-1)(1-a)} \frac{a}{n} \sum_{t=1}^{T-1} \sum_{i=1}^{n-1} y_{t i}=\frac{-K}{((T-1) K+1)(n-1)} \sum_{t=1}^{T-1} \sum_{i=1}^{n-1} y_{t i} .
$$

Combinando essas três expressões, o BLUP de $y_{T n}$ é dado por

$$
\widehat{y}_{T n}=\bar{y}_{T \cdot}+\left(\frac{K(T-1)}{K(T-1)+1}\right)\left(\bar{y}_{\cdot n}-\bar{y}_{c}\right) \text {. }
$$

\section{B.2. OBTENÇÃO DO BLUP POR MULTIPLICADORES DE LAGRANGE}

Usando multiplicadores de Lagrange, queremos minimizar $f$, dada por

$$
f=S^{2} \sum_{i=1}^{n} \tilde{c}_{. i}^{2}+\sigma^{2} \sum_{i=1}^{n} \sum_{t=1}^{T} \bar{c}_{t i}^{2}+\sum_{t=1}^{T-1} \lambda_{t} \sum_{i=1}^{n} c_{t i}+\lambda_{T}\left(\sum_{i=1}^{n-1} c_{T i}-1\right)+\lambda_{T+1} c_{T n}
$$


Os coeficientes que minimizam $f$ são dados pela solução do sistema de $T(n+1)$ equações abaixo, gerado pelas derivadas de $f$. Assim,

1) $\frac{\partial f}{\partial c_{T j}}=2 S^{2} \tilde{c}_{. j} \frac{\partial \tilde{c}_{. j}}{\partial c_{T j}}+2 \sigma^{2} \bar{c}_{T j} \frac{\partial \bar{c}_{T j}}{\partial c_{T j}}+\lambda_{T}$

$$
=2 S^{2} \sum_{t=1}^{T} c_{t j}+2 \sigma^{2} c_{T j}+\lambda_{T}=0
$$

$(n-1$ equações correspondentes a $t=T$ e $j=1,2, \ldots, n-1)$

2) $\frac{\partial f}{\partial c_{v n}}=2 S^{2} \tilde{c}_{. n} \frac{\partial \tilde{c}_{. n}}{\partial c_{v n}}+2 \sigma^{2} \bar{c}_{v n} \frac{\partial \bar{c}_{v n}}{\partial c_{v n}}+\lambda_{v}$

$$
\begin{aligned}
& =2 S^{2}\left(c_{. n}-1\right)+2 \sigma^{2} c_{v n}+\lambda_{v} \\
& =2 S^{2} \sum_{t=1}^{T} c_{t n}-2 S^{2}+2 \sigma^{2} c_{v n}+\lambda_{v}=0
\end{aligned}
$$

$(T-1$ equações correspondentes a $i=n$ e $v=1,2, \ldots, T-1)$

3) $\frac{\partial f}{\partial c_{v j}}=2 S^{2} \tilde{c}_{. j} \frac{\partial \tilde{c}_{. j}}{\partial c_{v j}}+2 \sigma^{2} \bar{c}_{v j} \frac{\partial \bar{c}_{v j}}{\partial c_{v j}}+\lambda_{v}$

$$
=2 S^{2} \sum_{t=1}^{T} c_{t j}+2 \sigma^{2} c_{v j}+\lambda_{v}=0
$$

$((n-1)(T-1)$ equações correspondentes a $j=1,2, \ldots, n-1$ e $v=1,2, \ldots, T-1)$

4) $\frac{\partial f}{\partial \lambda_{v}}=\sum_{i=1}^{n} c_{v i}=0$

$(T-1)$ equações correspondentes a $v=1,2, \ldots, T-1)$

5) $\frac{\partial f}{\partial \lambda_{T}}=\sum_{i=1}^{n-1} c_{T i}-1=0$

6) $\frac{\partial f}{\partial \lambda_{T+1}}=c_{T n}=0$ 
cuja solução é

$$
\begin{aligned}
& c_{T i} \quad=\frac{1}{n-1}, \quad i=1,2, \ldots, n-1 \\
& c_{t n}=\frac{K}{K(T-1)+1}, \quad t=1,2, \ldots, T-1 \\
& c_{t i}=-\frac{K}{(K(T-1)+1)(n-1)}, \quad i=1,2, \ldots, n-1 \quad \text { e } t=1,2, \ldots, T-1 \\
& c_{T n}=0, \\
& \lambda_{t} \quad=0, \quad t=1,2, \ldots, T-1 \\
& \lambda_{T} \quad=-2 S^{2}\left(\frac{1}{(K(T-1)+1)(n-1)}\right)-\frac{2 \sigma^{2}}{n-1}, \\
& \lambda_{T+1} \quad=0,
\end{aligned}
$$

que leva ao previsor dado por (3.9).

Verificação da solução do sistema de equações

(1) Para qualquer $j=1,2, \ldots, n-1$

$$
\begin{aligned}
& 2 S^{2} \sum_{t=1}^{T} c_{t j}+2 \sigma^{2} c_{T j}+\lambda_{T} \\
& =2 S^{2}\left(\frac{-K(T-1)}{(K(T-1)+1)(n-1)}+\frac{1}{(n-1)}\right)+\frac{2 \sigma^{2}}{(n-1)}-2 S^{2}\left(\frac{1}{(K(T-1)+1)(n-1)}\right)-\frac{2 \sigma^{2}}{(n-1)}=0 .
\end{aligned}
$$

(2) Para qualquer $v=1,2, \ldots, T-1$

$$
\begin{aligned}
& 2 S^{2} \sum_{t=1}^{T} c_{t n}-2 S^{2}+2 \sigma^{2} c_{v n}+\lambda_{v} \\
& =2 S^{2}\left(\frac{K(T-1)}{K(T-1)+1}\right)-2 S^{2}+2 \sigma^{2} \frac{K}{K(T-1)+1}
\end{aligned}
$$


$=2 S^{2}\left(\frac{K(T-1)-K(T-1)-1}{K(T-1)+1}\right)+\frac{2 S^{2}}{K(T-1)+1}=0$.

(3) Para qualquer $j=1,2, \ldots, n-1$ e qualquer $v=1,2, \ldots, T-1$

$$
\begin{aligned}
& 2 S^{2} \sum_{t=1}^{T} c_{t j}+2 \sigma^{2} c_{v j}+\lambda_{v} \\
& =2 S^{2}\left(\frac{-K(T-1)}{(K(T-1)+1)(n-1)}+\frac{1}{(n-1)}\right)+2 \sigma^{2}\left(\frac{-K}{(K(T-1)+1)(n-1)}\right) \\
& =2 S^{2}\left(\frac{1}{(K(T-1)+1)(n-1)}\right)-\frac{2 S^{2}}{(K(T-1)+1)(n-1)}=0 .
\end{aligned}
$$

(4) Para qualquer $v=1,2, \ldots, T-1$

$$
\sum_{i=1}^{n} c_{v i}=-\frac{(n-1) K}{(K(T-1)+1)(n-1)}+\frac{K}{(K(T-1)+1)}=0
$$

(5) $\sum_{i=1}^{n-1} c_{T_{i}}-1=\frac{(n-1)}{(n-1)}-1=0$.

(6) $c_{T n}=0$.

\section{B.3. $m$ OBSERVAÇÕES PERDIDAS NO ÚLTIMO INSTANTE}

Como no caso anterior,

$$
\mathbf{Q}=\frac{1}{n}\left(\mathbf{J}_{n} \otimes \mathbf{I}_{T}\right)+a\left(\mathbf{I}_{n} \otimes \mathbf{J}_{T}\right)-\frac{a}{n}\left(\mathbf{J}_{n} \otimes \mathbf{J}_{T}\right)
$$

com

$$
a=\frac{K}{T K+1}, \quad K=\frac{S^{2}}{\sigma^{2}}, \quad \mathrm{E}^{\prime} \mathrm{E}=\mathrm{I}_{m}, \quad \mathrm{FF}^{\prime}=\left(\begin{array}{cc}
\mathrm{A} & 0 \\
0 & \mathrm{~B}
\end{array}\right)
$$


onde $\mathbf{A}=\mathrm{I}_{(n-m)} \otimes \mathrm{I}_{T} \quad$ e $\quad \mathrm{B}=\left(\begin{array}{cc}\mathrm{I}_{(T-1)} & 0 \\ 0 & 0\end{array}\right) \otimes \mathrm{I}_{m}$

$$
\begin{aligned}
& \mathbf{E}^{\prime} \mathrm{Q}=\left(\mathbf{1}_{m} \mathbf{1}_{n}^{\prime}\right) \otimes(0,0, \ldots, 1 / n)+\left(\begin{array}{ll}
0 & a \mathrm{I}_{m}
\end{array}\right) \otimes \mathbf{1}_{T}^{\prime}-\frac{a}{n} \mathbf{1}_{m} \mathbf{1}_{n T}^{\prime} \\
& \mathrm{E}^{\prime} \mathrm{QE}=a \mathrm{I}_{m}+\frac{1-a}{n} \mathrm{~J}_{m} \\
& \mathrm{I}_{m}-\mathrm{E}^{\prime} \mathrm{QE}=(1-a)\left(\mathrm{I}_{m}-\frac{1}{n} \mathrm{~J}_{m}\right) \\
& \left(\mathrm{I}_{m}-\mathrm{E}^{\prime} \mathrm{QE}\right)^{-1}=\frac{1}{(1-a)} \mathrm{I}_{m}+\frac{1}{(n-m)(1-a)} \mathrm{J}_{m} \\
& \mathrm{E}^{\prime} \mathrm{QFF}=\left(\begin{array}{llll}
\mathrm{C}_{1} & \mathrm{C}_{2} & \cdots & \mathrm{C}_{n}
\end{array}\right)
\end{aligned}
$$

onde

para $i=1,2, \ldots, n-m$,

$$
\underset{(m \times T)}{\mathrm{C}_{i}}=\left(\begin{array}{ccccc}
-\frac{a}{n} & -\frac{a}{n} & \cdots & -\frac{a}{n} & \frac{1-a}{n} \\
-\frac{a}{n} & -\frac{a}{n} & \cdots & -\frac{a}{n} & \frac{1-a}{n} \\
\vdots & \vdots & \vdots & \vdots & \vdots \\
-\frac{a}{n} & -\frac{a}{n} & \cdots & -\frac{a}{n} & \frac{1-a}{n}
\end{array}\right)
$$

e para $j=1,2, \ldots, m$.

$$
\mathrm{C}_{(m \times T)}+j=\left(\begin{array}{ccccc}
-\frac{a}{n} & -\frac{a}{n} & \cdots & -\frac{a}{n} & 0 \\
-\frac{a}{n} & -\frac{a}{n} & \cdots & -\frac{a}{n} & 0 \\
\vdots & \vdots & \vdots & \vdots & \vdots \\
\frac{(n-1) a}{n} & \frac{(n-1) a}{n} & \cdots & \frac{(n-1) a}{n} & 0 \\
\vdots & \vdots & \vdots & \vdots & \vdots \\
-\frac{a}{n} & -\frac{a}{n} & \cdots & -\frac{a}{n} & 0
\end{array}\right) \rightarrow j \text {-ésima linha }
$$

A multiplicação de $\left(I_{m}-E^{\prime} Q E\right)^{-1}$ por $E^{\prime} Q F F^{\prime}$ leva ao BLUP, dado por:

$$
\widehat{y}_{T i}=\bar{y}_{T .}+\left(\frac{K(T-1)}{K(T-1)+1}\right)\left(\bar{y}_{\cdot i}-\bar{y}_{c}\right), \text { para } i=n-m+1, \ldots, n .
$$




\section{B.4. EXEMPLO}

Três indivíduos são observados em três instantes. Para dois deles, a última observação é perdida. Assim, $n=3 T=3 \quad m=2$

Dados

$$
\begin{aligned}
& \begin{array}{c|cc|c}
\text { indivíduo } & \multicolumn{3}{|c}{\text { tempo }} \\
& 1 & 2 & 3 \\
\hline 1 & y_{11} & y_{21} & y_{31} \\
2 & y_{12} & y_{22} & ? \\
3 & y_{13} & y_{23} & ?
\end{array} \\
& \mathbf{Q}=\frac{1}{n}\left(\mathbf{J}_{n} \otimes \mathbf{I}_{T}\right)+a\left(\mathbf{I}_{n} \otimes \mathbf{J}_{T}\right)-\frac{a}{n}\left(\mathbf{J}_{n} \otimes \mathbf{J}_{T}\right) \\
& =\left(\begin{array}{ccccccccc}
\frac{1}{n} & 0 & 0 & \frac{1}{n} & 0 & 0 & \frac{1}{n} & 0 & 0 \\
0 & \frac{1}{n} & 0 & 0 & \frac{1}{n} & 0 & 0 & \frac{1}{n} & 0 \\
0 & 0 & \frac{1}{n} & 0 & 0 & \frac{1}{n} & 0 & 0 & \frac{1}{n} \\
& & & & & & & & \\
\frac{1}{n} & 0 & 0 & \frac{1}{n} & 0 & 0 & \frac{1}{n} & 0 & 0 \\
0 & \frac{1}{n} & 0 & 0 & \frac{1}{n} & 0 & 0 & \frac{1}{n} & 0 \\
0 & 0 & \frac{1}{n} & 0 & 0 & \frac{1}{n} & 0 & 0 & \frac{1}{n} \\
\frac{1}{n} & 0 & 0 & \frac{1}{n} & 0 & 0 & \frac{1}{n} & 0 & 0 \\
0 & \frac{1}{n} & 0 & 0 & \frac{1}{n} & 0 & 0 & \frac{1}{n} & 0 \\
0 & 0 & \frac{1}{n} & 0 & 0 & \frac{1}{n} & 0 & 0 & \frac{1}{n}
\end{array}\right)+\left(\begin{array}{ccccccccc}
a & a & a & 0 & 0 & 0 & 0 & 0 & 0 \\
a & a & a & 0 & 0 & 0 & 0 & 0 & 0 \\
a & a & a & 0 & 0 & 0 & 0 & 0 & 0 \\
& & & & & & & & \\
0 & 0 & 0 & a & a & a & 0 & 0 & 0 \\
0 & 0 & 0 & a & a & a & 0 & 0 & 0 \\
0 & 0 & 0 & a & a & a & 0 & 0 & 0 \\
& & & & & & & & \\
0 & 0 & 0 & 0 & 0 & 0 & a & a & a \\
0 & 0 & 0 & 0 & 0 & 0 & a & a & a \\
0 & 0 & 0 & 0 & 0 & 0 & a & a & a
\end{array}\right) \\
& -\frac{a}{n} \mathrm{~J}_{n T} \\
& \mathbf{E}^{\prime}=\left(\begin{array}{ccccccccc}
0 & 0 & 0 & 0 & 0 & 1 & 0 & 0 & 0 \\
0 & 0 & 0 & 0 & 0 & 0 & 0 & 0 & 1
\end{array}\right) \quad \mathbf{E}^{\prime} \mathbf{E}=\left(\begin{array}{ll}
1 & 0 \\
0 & 1
\end{array}\right)=\mathbf{I}_{2}
\end{aligned}
$$




$$
\mathbf{F}=\left(\begin{array}{ccccccc}
1 & 0 & 0 & 0 & 0 & 0 & 0 \\
0 & 1 & 0 & 0 & 0 & 0 & 0 \\
0 & 0 & 1 & 0 & 0 & 0 & 0 \\
0 & 0 & 0 & 1 & 0 & 0 & 0 \\
0 & 0 & 0 & 0 & 1 & 0 & 0 \\
0 & 0 & 0 & 0 & 0 & 0 & 0 \\
& & & & & & \\
0 & 0 & 0 & 0 & 0 & 1 & 0 \\
0 & 0 & 0 & 0 & 0 & 0 & 1 \\
0 & 0 & 0 & 0 & 0 & 0 & 0
\end{array}\right) \quad F^{\prime}=\left(\begin{array}{ccccccccc}
1 & 0 & 0 & 0 & 0 & 0 & 0 & 0 & 0 \\
0 & 1 & 0 & 0 & 0 & 0 & 0 & 0 & 0 \\
0 & 0 & 1 & 0 & 0 & 0 & 0 & 0 & 0 \\
& & & & & & & & \\
0 & 0 & 0 & 1 & 0 & 0 & 0 & 0 & 0 \\
0 & 0 & 0 & 0 & 1 & 0 & 0 & 0 & 0 \\
0 & 0 & 0 & 0 & 0 & 0 & 0 & 0 & 0 \\
& & & & & & & & \\
0 & 0 & 0 & 0 & 0 & 0 & 1 & 0 & 0 \\
0 & 0 & 0 & 0 & 0 & 0 & 0 & 1 & 0 \\
0 & 0 & 0 & 0 & 0 & 0 & 0 & 0 & 0
\end{array}\right)
$$

$$
\begin{aligned}
& \mathbf{E}^{\prime} \mathbf{Q}=\left(\begin{array}{lllllllll}
0 & 0 & \frac{1}{n} & 0 & 0 & \frac{1}{n} & 0 & 0 & \frac{1}{n} \\
0 & 0 & \frac{1}{n} & 0 & 0 & \frac{1}{n} & 0 & 0 & \frac{1}{n}
\end{array}\right)+\left(\begin{array}{ccccccccc}
0 & 0 & 0 & a & a & a & 0 & 0 & 0 \\
0 & 0 & 0 & 0 & 0 & 0 & a & a & a
\end{array}\right) \\
& -\left(\begin{array}{lllllllll}
\frac{a}{n} & \frac{a}{n} & \frac{a}{n} & \frac{a}{n} & \frac{a}{n} & \frac{a}{n} & \frac{a}{n} & \frac{a}{n} & \frac{a}{n} \\
\frac{a}{n} & \frac{a}{n} & \frac{a}{n} & \frac{a}{n} & \frac{a}{n} & \frac{a}{n} & \frac{a}{n} & \frac{a}{n} & \frac{a}{n}
\end{array}\right)
\end{aligned}
$$

$$
\begin{aligned}
\mathrm{E}^{\prime} \mathrm{QE} & =\left(\begin{array}{cc}
\frac{1}{n} & \frac{1}{n} \\
\frac{1}{n} & \frac{1}{n}
\end{array}\right)+\left(\begin{array}{cc}
a & 0 \\
0 & a
\end{array}\right)-\left(\begin{array}{cc}
\frac{a}{n} & \frac{a}{n} \\
\frac{a}{n} & \frac{a}{n}
\end{array}\right) \\
& =a \mathrm{I}_{m}+\frac{1-a}{n} \mathrm{~J}_{m} \\
\mathrm{I}_{m}-\mathrm{E}^{\prime} \mathrm{QE} & =\mathrm{I}_{m}-a \mathrm{I}_{m}-\frac{(1-a)}{n} \mathrm{~J}_{m} \\
& =(1-a) \mathrm{I}_{m}-\frac{(1-a)}{n} \mathrm{~J}_{m}=(1-a)\left[\mathrm{I}_{m}-\frac{1}{n} \mathrm{~J}_{m}\right] \\
\left(\mathrm{I}_{m}-\mathrm{E}^{\prime} \mathrm{QE}\right)^{-1} & =\frac{1}{(1-a)}\left[\mathrm{I}_{m}+\frac{\frac{1}{n}}{\left(-\frac{m}{n}+1\right)} \mathrm{J}_{m}\right] \\
& =\frac{1}{(1-a)} \mathrm{I}_{m}+\frac{1}{(n-m)(1-a)} \mathrm{J}_{m}
\end{aligned}
$$

$\mathrm{E}^{\prime} \mathrm{QFF}^{\prime}=\left(\begin{array}{ccccccccc}0 & 0 & \frac{1}{n} & 0 & 0 & 0 & 0 & 0 & 0 \\ 0 & 0 & \frac{1}{n} & 0 & 0 & 0 & 0 & 0 & 0\end{array}\right)+\left(\begin{array}{ccccccccc}0 & 0 & 0 & a & a & 0 & 0 & 0 & 0 \\ 0 & 0 & 0 & 0 & 0 & 0 & a & a & 0\end{array}\right)$ 


$$
\begin{aligned}
& -\left(\begin{array}{ccccccccc}
\frac{a}{n} & \frac{a}{n} & \frac{a}{n} & \frac{a}{n} & \frac{a}{n} & 0 & \frac{a}{n} & \frac{a}{n} & 0 \\
\frac{a}{n} & \frac{a}{n} & \frac{a}{n} & \frac{a}{n} & \frac{a}{n} & 0 & \frac{a}{n} & \frac{a}{n} & 0
\end{array}\right) \\
= & \left(\begin{array}{ccccccccc}
-\frac{a}{n} & -\frac{a}{n} & \frac{1-a}{n} & a-\frac{a}{n} & a-\frac{a}{n} & 0 & -\frac{a}{n} & -\frac{a}{n} & 0 \\
-\frac{a}{n} & -\frac{a}{n} & \frac{1-a}{n} & -\frac{a}{n} & -\frac{a}{n} & 0 & a-\frac{a}{n} & a-\frac{a}{n} & 0
\end{array}\right)
\end{aligned}
$$

\section{Previsor}

\section{1 - dado perdido}

$$
\begin{aligned}
& \frac{1}{(1-a)}\left(-\frac{a}{n},-\frac{a}{n}, \frac{1-a}{n}, \frac{(n-1) a}{n}, \frac{(n-1) a}{n}, 0,-\frac{a}{n},-\frac{a}{n}, 0\right) \\
& +\frac{1}{(1-a)(n-m)}\left(-\frac{m a}{n},-\frac{m a}{n}, \frac{m(1-a)}{n}, \frac{(n-m) a}{n}, \frac{(n-m) a}{n}, 0, \frac{(n-m) a}{n}, \frac{(n-m) a}{n}, 0\right) \\
& =\frac{1}{(1-a)(n-m)}(-a,-a,(1-a),(n-m) a,(n-m) a, 0,0,0,0) . \\
& \quad \operatorname{Mas} \frac{a}{1-a}=\frac{K}{(T-1) K+1} \mathrm{e} \\
& \hat{y}_{32}=\frac{1}{(n-m)} y_{31}+\frac{K}{(T-1) K+1}\left(y_{12}+y_{22}\right)-\frac{K}{(T-1) K+1} \frac{1}{(n-m)}\left(y_{11}+y_{21}\right) \\
& \quad=y_{31}+\frac{2 K}{2 K+1} \bar{y}_{\cdot 2}-\frac{2 K}{2 K+1} \bar{y}_{\cdot 1}
\end{aligned}
$$

ou seja

$$
\widehat{y}_{32}=y_{31}+\frac{2 K}{2 K+1}\left(\bar{y}_{\cdot 2}-\bar{y}_{\cdot 1}\right)
$$

\section{$2^{\circ}$ dado perdido}

Analogamente à derivação de $\hat{y}_{32}$, temos

$$
\begin{aligned}
& \frac{1}{(1-a)}\left(-\frac{a}{n},-\frac{a}{n}, \frac{1-a}{n},-\frac{a}{n},-\frac{a}{n}, 0, \frac{(n-1) a}{n}, \frac{(n-1) a}{n}, 0\right) \\
& +\frac{1}{(1-a)(n-m)}\left(-\frac{m a}{n},-\frac{m a}{n}, \frac{m(1-a)}{n}, \frac{(n-m) a}{n}, \frac{(n-m) a}{n}, 0, \frac{(n-m) a}{n}, \frac{(n-m) a}{n}, 0\right) \\
& =\frac{1}{(1-a)(n-m)}(-a,-a,(1-a), 0,0,0,(n-m) a,(n-m) a, 0)
\end{aligned}
$$

$$
\widehat{y}_{33}=y_{31}+\frac{2 K}{2 K+1}\left(\bar{y}_{\cdot 3}-\bar{y}_{\cdot 1}\right)
$$




\section{B.5. EXEMPLO DA OBTENÇÃO DO BLUP PELO USO DE MULTIPLICADORES DE LAGRANGE}

Suponhamos a seguinte situação: três indivíduos observados em três instantes, com duas observações perdidas, uma do segundo indivíduo no segundo instante e a outra do terceiro indivíduo no terceiro instante. O esquema representativo dos dados seria:

$$
\begin{aligned}
& \begin{array}{lll}
y_{11} & y_{21} & y_{31}
\end{array} \\
& \begin{array}{lll}
y_{12} & ? & y_{32}
\end{array} \\
& y_{13} \quad y_{23} \quad ? \\
& \mathbf{E}^{\prime}=\left(\begin{array}{ccccccccc}
0 & 0 & 0 & 0 & 1 & 0 & 0 & 0 & 0 \\
0 & 0 & 0 & 0 & 0 & 0 & 0 & 0 & 1
\end{array}\right) \\
& X=\left(1_{3} \otimes I_{3}\right) \\
& \mathrm{W}=\left[\left(\mathrm{I}_{3} \otimes \mathrm{I}_{3}\right)+K\left(\mathrm{I}_{3} \otimes \mathrm{J}_{3}\right)-\frac{K}{N}\left(\mathrm{~J}_{3} \otimes \mathrm{J}_{3}\right)\right]
\end{aligned}
$$

onde $K=\frac{S^{2}}{\sigma^{2}}$.

A matriz $\mathbf{C}$ é uma matriz de dimensão $(2 \times 9)$ dada por

$$
\mathrm{C}=\left(\begin{array}{lllllllll}
c_{111} & c_{121} & c_{131} & c_{112} & c_{122} & c_{132} & c_{113} & c_{123} & c_{133} \\
c_{211} & c_{221} & c_{231} & c_{212} & c_{222} & c_{232} & c_{213} & c_{223} & c_{233}
\end{array}\right)
$$

onde $h=15 K^{2}+16 K+4$,

$$
\begin{aligned}
& c_{111}=-h\left(3 K^{2}+2 K\right), \quad c_{112}=h\left(9 K^{2}+4 K\right), \quad c_{113}=-h\left(6 K^{2}+2 K\right), \\
& c_{121}=h\left(9 K^{2}+8 K+2\right), \quad c_{123}=h\left(6 K^{2}+8 K+2\right), \quad c_{131}=-h\left(6 K^{2}+3 K\right), \\
& c_{132}=h\left(6 K^{2}+3 K\right)
\end{aligned}
$$

e

$$
\begin{aligned}
& c_{211}=-h\left(3 K^{2}+2 K\right), \quad c_{212}=-h\left(6 K^{2}+2 K\right), \quad c_{213}=h\left(9 K^{2}+4 K\right), \\
& c_{221}=-h\left(6 K^{2}+3 K\right), \quad c_{223}=h\left(6 K^{2}+3 K\right), \quad c_{231}=h\left(9 K^{2}+8 K+2\right), \\
& c_{232}=h\left(6 K^{2}+8 K+2\right) \text {. }
\end{aligned}
$$


Considerando $\Lambda_{i}^{\prime}=211^{\prime} \sigma^{2} \mathrm{~A}^{\prime}$ e $\Lambda_{2}^{\prime}=-211^{\prime} \sigma^{2} \mathrm{~B}^{\prime}$, a equação (3.18) passa a ser

$$
2 \mathrm{Il}^{\prime} \sigma^{2}\left(\mathrm{CW}-\mathrm{E}^{\prime} \mathrm{W}+\mathrm{A}^{\prime} \mathrm{E}^{\prime}-\mathrm{B}^{\prime} \mathrm{X}^{\prime}\right)=0,
$$

ou seja, queremos obter A e B que satisfaçam

$$
\mathrm{CW}-\mathrm{E}^{\prime} \mathrm{W}+\mathrm{A}^{\prime} \mathrm{E}^{\prime}-\mathrm{B}^{\prime} \mathrm{X}^{\prime}=0 .
$$

$$
\begin{aligned}
\text { Sejam } \mathbf{A}^{\prime} & =\left(\begin{array}{ll}
a_{11} & a_{12} \\
a_{21} & a_{22}
\end{array}\right) \text { e } \mathbf{B}^{\prime}=\left(\begin{array}{llll}
b_{11} & b_{12} & b_{13} \\
b_{21} & b_{22} & b_{23}
\end{array}\right) \text {. Então, } \\
\mathbf{B}^{\prime} \mathrm{X}^{\prime} & =\left(\begin{array}{lllllllll}
b_{11} & b_{12} & b_{13} & b_{11} & b_{12} & b_{13} & b_{11} & b_{12} & b_{13} \\
b_{21} & b_{22} & b_{23} & b_{21} & b_{22} & b_{23} & b_{21} & b_{22} & b_{23}
\end{array}\right) \text { e } \\
\mathbf{A}^{\prime} \mathrm{E}^{\prime} & =\left(\begin{array}{lllllllll}
0 & 0 & 0 & 0 & a_{11} & 0 & 0 & 0 & a_{12} \\
0 & 0 & 0 & 0 & a_{21} & 0 & 0 & 0 & a_{22}
\end{array}\right) .
\end{aligned}
$$

A e B podem ser obtidos pela equação (2), uma vez que $\left(C W-E^{\prime} W\right)^{\prime}$ é dada por

$$
\left(\mathrm{CW}-\mathrm{E}^{\prime} \mathrm{W}\right)^{\prime}=\left(\begin{array}{cc}
0 & 0 \\
h\left(12 K^{2}+10 K+2\right) & -h\left(3 K^{2}+K\right) \\
-h\left(3 K^{2}+K\right) & h\left(12 K^{2}+10 K+2\right) \\
0 & 0 \\
h\left(15 K^{3}+7 K^{2}-K-1\right) & h\left(6 K^{2}+2 K\right) \\
-h\left(3 K^{2}+K\right) & h\left(12 K^{2}+10 K+2\right) \\
0 & 0 \\
h\left(12 K^{2}+10 K+2\right) & -h\left(3 K^{2}+K\right) \\
h\left(6 K^{2}+2 K\right) & h\left(15 K^{3}+7 K^{2}-K-1\right)
\end{array}\right)
$$

Assim, os elementos de B e de A são

$$
\begin{aligned}
& b_{11}=b_{21}=0 \\
& b_{12}=b_{23}=h\left(12 K^{2}+10 K+2\right) \\
& b_{13}=b_{22}=-h\left(3 K^{2}+K\right)
\end{aligned}
$$


e

$$
\begin{aligned}
& a_{11}=a_{22}=h\left(-15 K^{3}+5 K^{2}+11 K+3\right) \\
& a_{12}=a_{21}=-3 h\left(3 K^{2}+K\right)
\end{aligned}
$$

o que significa que $\mathrm{A}$ e $\mathrm{B}$ podem ser expressos como

$$
\begin{aligned}
& \mathbf{B}^{\prime}=\left(\mathbf{C W}-\mathbf{E}^{\prime} \mathbf{W}\right)\left(\begin{array}{lll}
1 & 0 & 0 \\
0 & 1 & 0 \\
0 & 0 & 1 \\
0 & 0 & 0 \\
0 & 0 & 0 \\
0 & 0 & 0 \\
0 & 0 & 0 \\
0 & 0 & 0 \\
0 & 0 & 0
\end{array}\right) \\
& \mathbf{A}^{\prime}=\mathbf{B}^{\prime}\left(\begin{array}{ll}
0 & 0 \\
1 & 0 \\
0 & 1
\end{array}\right)-\left(\begin{array}{ll}
1 & 0 \\
0 & 0
\end{array}\right)\left(\mathbf{C W}-\mathbf{E}^{\prime} \mathbf{W}\right)\left(\begin{array}{ll}
0 & 0 \\
0 & 0 \\
0 & 0 \\
0 & 0 \\
1 & 0 \\
0 & 1 \\
0 & 0 \\
0 & 0 \\
0 & 0
\end{array}\right)-\left(\begin{array}{ll}
0 & 0 \\
0 & 1
\end{array}\right)\left(\mathbf{C W}-\mathbf{E}^{\prime} \mathbf{W}\right)\left(\begin{array}{ll}
0 & 0 \\
0 & 0 \\
0 & 0 \\
0 & 0 \\
0 & 0 \\
0 & 0 \\
0 & 0 \\
1 & 0 \\
0 & 1
\end{array}\right)
\end{aligned}
$$

\section{B.6. CÁLCULO DO ERRO QUADRÁTICO MÉDIO DE $\hat{y}_{T n}$}

Seja

$$
\begin{aligned}
x_{t i} & =y_{t i}-\mu_{t}=b_{i}+e_{t i} \\
\hat{y}_{T n}-\mu_{T} & =\bar{y}_{T \cdot}-\mu_{T}+R\left(\bar{y}_{\cdot n}-\bar{y}_{c}\right) \\
& =\frac{1}{n-1} \sum_{i=1}^{n-1} y_{T i}-\mu_{T}+R\left(\frac{1}{T-1} \sum_{t=1}^{T-1} y_{t n}-\frac{1}{(n-1)(T-1)} \sum_{i=1}^{n-1} \sum_{t=1}^{T-1} y_{t i}\right) \\
& =\frac{1}{n-1} \sum_{i=1}^{n-1} x_{T i}+R\left(\frac{1}{T-1} \sum_{t=1}^{T-1} x_{t n}-\frac{1}{(n-1)(T-1)} \sum_{i=1}^{n-1} \sum_{t=1}^{T-1} x_{t i}\right) \\
& =\bar{x}_{T \cdot}+R\left(\bar{x}_{\cdot n}-\bar{x}_{c}\right)=\widehat{x}_{T n} .
\end{aligned}
$$


Para obter o erro quadrático médio de $\widehat{y}_{T n}$, precisamos de algumas variâncias e covariâncias. Então,

$$
\begin{aligned}
& \operatorname{Var}\left(x_{T n}\right)=\operatorname{Var}\left(b_{T}+e_{T n}\right)=\frac{(N-1)}{N} S^{2}+\sigma^{2}
\end{aligned}
$$

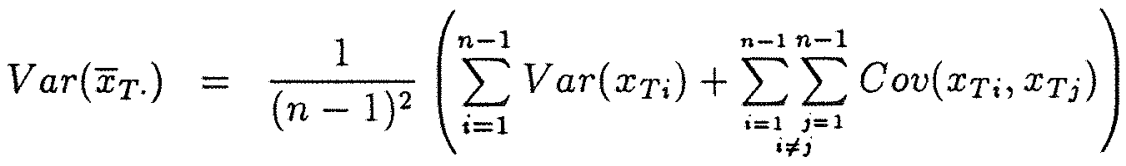

$$
\begin{aligned}
& =\frac{1}{(n-1)^{2}}\left(\sum_{i=1}^{n-1}\left(\frac{N-1}{N} S^{2}+\sigma^{2}\right)+\sum_{i=1}^{n-1} \sum_{\substack{i \neq j \\
i \neq 1}}^{n-1}\left(-\frac{S^{2}}{N}\right)\right) \\
& =\frac{(N-1)}{N(n-1)} S^{2}+\frac{1}{n-1} \sigma^{2}-\frac{(n-2)}{(n-1) N} S^{2}=\frac{N-(n-1)}{N(n-1)} S^{2}+\frac{\sigma^{2}}{n-1} \\
& \operatorname{Var}\left(\bar{x}_{. n}\right)=\frac{1}{(T-1)^{2}}\left(\sum_{t=1}^{T-1} \operatorname{Var}\left(x_{t n}\right)+\sum_{t=1}^{T-1} \sum_{\substack{v=1 \\
t \neq v}}^{T-1} \operatorname{Cov}\left(x_{t n}, x_{v n}\right)\right) \\
& =\frac{1}{(T-1)^{2}}\left(\sum_{t=1}^{T-1}\left(\frac{N-1}{N} S^{2}+\sigma^{2}\right)+\sum_{\substack{t=1 \\
t \neq v}}^{T-1} \sum_{\substack{t-1 \\
T}}^{T-1} \frac{N-1}{N} S^{2}\right) \\
& =\frac{(N-1)}{N(T-1)} S^{2}+\frac{\sigma^{2}}{(T-1)}+\frac{(T-2)(N-1)}{N(T-1)} S^{2}=\frac{(N-1)}{N} S^{2}+\frac{\sigma^{2}}{(T-1)} \\
& \operatorname{Var}\left(\bar{x}_{c}\right)=\frac{1}{(n-1)^{2}(T-1)^{2}}\left(\sum_{t=1}^{T-1} \sum_{i=1}^{n-1} \operatorname{Var}\left(x_{t i}\right)+\sum_{i=1}^{T-1} \sum_{\substack{i=1 \\
i \neq j}}^{n-1} \sum_{j=1}^{n-1} \operatorname{Cov}\left(x_{t i}, x_{t j}\right)\right.
\end{aligned}
$$

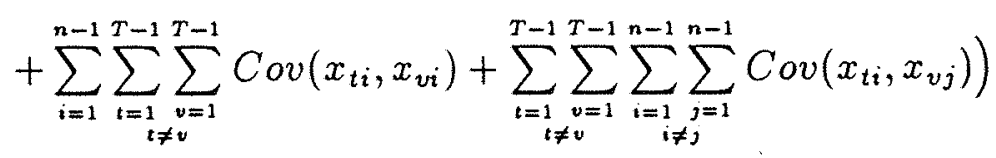

$$
\begin{aligned}
& =\frac{(N-1)}{N(n-1)(T-1)} S^{2}+\frac{\sigma^{2}}{(n-1)(T-1)}-\frac{(n-2)}{(T-1)(n-1) N} S^{2} \\
& +\frac{(N-1)(T-2)}{N(T-1)(n-1)} S^{2}-\frac{(n-2)(T-2)}{N(n-1)(T-1)} S^{2} \\
& =\frac{N-(n-1)}{N(n-1)} S^{2}+\frac{\sigma^{2}}{(n-1)(T-1)}
\end{aligned}
$$




$$
\begin{aligned}
\operatorname{Cov}\left(\bar{x}_{T \cdot}, \bar{x}_{\cdot n}\right) & =\frac{1}{(n-1)(T-1)} \operatorname{Cov}\left(\sum_{i=1}^{n-1} x_{T i}, \sum_{t=1}^{T-1} x_{t n}\right)=-\frac{S^{2}}{N} \\
\operatorname{Cov}\left(\bar{x}_{T .}, \bar{x}_{c}\right) & =\frac{1}{(n-1)^{2}(T-1)} \operatorname{Con}\left(\sum_{i=1}^{n-1} x_{T i}, \sum_{t=1}^{T-1} \sum_{j=1}^{n-1} x_{t j}\right) \\
& =\frac{(N-1)}{N(n-1)} S^{2}-\frac{(n-2)}{(n-1) N} S^{2}=\frac{N-(n-1)}{N(n-1)} S^{2} \\
\operatorname{Cov}\left(\bar{x}_{\cdot n}, \bar{x}_{c}\right) & =\frac{1}{(T-1)^{2}(n-1)} \operatorname{Cov}\left(\sum_{t=1}^{T-1} x_{t n}, \sum_{v=1}^{T-1} \sum_{i=1}^{n-1} x_{v i}\right)=-\frac{S^{2}}{N} \\
\operatorname{Cov}\left(\bar{x}_{T \cdot}, x_{T n}\right) & =\frac{1}{(n-1)} \operatorname{Cov}\left(\sum_{i=1}^{n-1} x_{T i}, x_{T n}\right)=-\frac{S^{2}}{N} \\
\operatorname{Cov}\left(\bar{x}_{\cdot n}, x_{T n}\right) & =\frac{1}{(T-1)} \operatorname{Cov}\left(\sum_{t=1}^{T-1} x_{t n}, x_{T n}\right)=\frac{(N-1)}{N} S^{2} \\
\operatorname{Cov}\left(\bar{x}_{c}, x_{T n}\right) & =\frac{1}{(n-1)(T-1)} \operatorname{Cov}\left(\sum_{t=1}^{T-1} \sum_{i=1}^{n-1} x_{t i}, x_{T n}\right)=-\frac{S^{2}}{N} .
\end{aligned}
$$

Assim,

$$
\widehat{y}_{T_{n}}=\bar{y}_{T}+R\left(\bar{y}_{\cdot n}-\bar{y}_{c}\right)
$$

onde

$$
R=\frac{K(T-1)}{K(T-1)+1}=\frac{S^{2}(T-1)}{S^{2}(T-1)+\sigma^{2}}
$$




$$
\begin{aligned}
& E Q M\left(\widehat{y}_{T n}\right)=\mathbb{E}\left[\left(\hat{y}_{T_{n}}-y_{T_{n}}\right)^{2}\right]=\mathbb{E}\left[\left(\left(\hat{y}_{T_{n}}-\mu_{T}\right)-\left(y_{T_{n}}-\mu_{T}\right)\right)^{2}\right] \\
& =\mathbb{E}\left[\left(\widehat{y}_{T_{n}}-\mu_{T}\right)^{2}\right]-2 \mathbb{E}\left[\left(\hat{y}_{T_{n}}-\mu_{T}\right)\left(y_{T_{n}}-\mu_{T}\right)\right]+\mathbb{E}\left[\left(y_{T_{n}}-\mu_{T}\right)^{2}\right] \\
& =\mathbb{E}\left[\left(\hat{x}_{T n}\right)^{2}\right]-2 \mathbb{E}\left[\hat{x}_{T n} x_{T n}\right]+\mathbb{E}\left[\left(\sim_{T n}\right)^{2}\right] \\
& =\operatorname{Var}\left(\widehat{x}_{T_{n}}\right)-2 \operatorname{Cov}\left(\widehat{x}_{T_{n}}, x_{T_{n}}\right)+\operatorname{Var}\left(x_{T_{n}}\right) \\
& =\operatorname{Var}\left(\bar{x}_{T \cdot}\right)+R^{2} \operatorname{Var}\left(\bar{x}_{\cdot n}\right)+R^{2} \operatorname{Var}\left(\bar{x}_{\mathrm{c}}\right) \\
& +2 R \operatorname{Cov}\left(\bar{x}_{T,}, \bar{x}_{\cdot n}\right)-2 R \operatorname{Cov}\left(\bar{x}_{T}, \bar{x}_{c}\right)-2 R^{2} \operatorname{Cov}\left(\bar{x}_{\cdot n}, \bar{x}_{c}\right) \\
& -2 \operatorname{Cov}\left(\bar{x}_{T .}, x_{T_{n}}\right)-2 R \operatorname{Cov}\left(\bar{x}_{\cdot n}, x_{T_{n}}\right)+2 R \operatorname{Cov}\left(\bar{x}_{c}, x_{T_{n}}\right)+\operatorname{Var}\left(x_{T_{n}}\right) \\
& =\frac{N-(n-1)}{N(n-1)} S^{2}+\frac{\sigma^{2}}{n-1}+R^{2}\left(\frac{(N-1)}{N} S^{2}+\frac{\sigma^{2}}{(T-1)}+\frac{N-(n-1)}{N(n-1)} S^{2}\right. \\
& \left.+\frac{\sigma^{2}}{(n-1)(T-1)}+2 \frac{S^{2}}{N}\right)+2 R\left(-\frac{S^{2}}{N}-\frac{N-(n-1)}{N(n-1)} S^{2}-\frac{(N-1)}{N} S^{2}-\frac{S^{2}}{N}\right) \\
& +2 \frac{S^{2}}{N}+\frac{(N-1)}{N} S^{2}+\sigma^{2} \\
& =\frac{n}{n-1}\left(1-2 R+R^{2}\right) S^{2}+\frac{n}{n-1}\left(1+\frac{R^{2}}{T-1}\right) \sigma^{2} .
\end{aligned}
$$

Substituindo $R$ nessa expressão, temos

$$
\operatorname{EQM}\left(\widehat{y}_{T n}\right)=\frac{n}{n-1}\left[\frac{K T+1}{K(T-1)+1}\right] \sigma^{2}
$$




\section{APÊNDICE C}

\section{CASO MULTIVARIADO}

\section{C.1. CÁlCULO DA INVERSA DE V}

$$
\mathrm{V}=\left(\mathrm{I}_{n} \otimes \mathrm{J}_{T} \otimes \mathrm{S}\right)-\frac{1}{N}\left(\mathrm{~J}_{n} \otimes \mathrm{J}_{T} \otimes \mathrm{S}\right)+\left(\mathrm{I}_{n} \otimes \mathrm{I}_{T} \otimes \mathrm{\Sigma}\right)
$$

$\mathrm{V}:$ matriz de dimensão $(n p T \times n p T)$.

$\mathrm{S}$ e $\boldsymbol{\Sigma}$ : matrizes de dimensão $(p \times p)$, de posto completo.

$\mathrm{A}$ inversa de $\mathrm{V}$ é obtida pela aplicação do resultado $\mathrm{A} .3$ do apêndice $\mathrm{A}$.

Seja $f=\frac{n}{N}$.

$$
\mathrm{V}=\mathrm{I}_{n} \otimes\left(\mathrm{J}_{T} \otimes \mathrm{S}+\mathrm{I}_{T} \otimes \boldsymbol{\Sigma}\right)+\mathrm{J}_{n} \otimes-\frac{1}{N} \mathrm{~J}_{T} \otimes \mathrm{S}
$$

Identificando $\mathbf{B}=\left(\mathrm{J}_{T} \otimes \mathrm{S}\right)+\left(\mathrm{I}_{T} \otimes \boldsymbol{\Sigma}\right)$ e $\mathrm{C}=-\frac{1}{N} \mathrm{~J}_{T} \otimes \mathrm{S}$, temos que 


$$
\begin{aligned}
& \mathrm{B}^{-1}=\left(\mathrm{I}_{T} \otimes \Sigma^{-1}\right)-\mathrm{J}_{T} \otimes(T \mathrm{~S}+\Sigma)^{-1} \mathrm{~S} \Sigma^{-1} \\
& n \mathbf{C}+\mathbf{B}=-f \mathbf{J}_{T} \otimes \mathbf{S}+\mathbf{J}_{2} \otimes \mathbf{S}+\mathbf{I}_{T} \otimes \boldsymbol{\Sigma} \\
& =\mathbf{I}_{T} \otimes \Sigma+\mathbf{J}_{T} \otimes(1-f) \mathrm{S} \\
& (n \mathbf{C}+\mathbf{B})^{-1}=\left(\mathbf{I}_{T} \otimes \Sigma^{-1}\right)-\mathbf{J}_{T} \otimes(T(1-f) \mathbf{S}+\Sigma)^{-1}(1-f) \mathbf{S} \Sigma^{-1} \\
& (n \mathrm{C}+\mathrm{B})^{-1} \mathrm{CB}^{-1}=\left[\left(\mathbf{I}_{T} \otimes \boldsymbol{\Sigma}^{-1}\right)-\mathrm{J}_{T} \otimes(T(1-f) \mathrm{S}+\boldsymbol{\Sigma})^{-1}(1-f) \mathrm{S} \boldsymbol{\Sigma}^{-1}\right] \\
& \cdot\left(-\frac{1}{N} \mathbf{J}_{T} \otimes \mathrm{S}\right)\left[\mathrm{I}_{T} \otimes \mathbf{\Sigma}^{-1}-\mathrm{J}_{T} \otimes(T \mathrm{~S}+\boldsymbol{\Sigma})^{-1} \mathbf{S} \Sigma^{-1}\right] \\
& =\mathrm{J}_{T} \otimes-\frac{1}{N} \Sigma^{-1} \mathrm{~S} \Sigma^{-1}+\mathrm{J}_{T} \otimes \frac{T}{N} \Sigma^{-1} \mathrm{~S}(T \mathrm{~S}+\Sigma)^{-1} \mathrm{~S} \Sigma^{-1} \\
& +\mathbf{J}_{T} \otimes \frac{T}{N}(T(1-f) \mathbf{S}+\boldsymbol{\Sigma})^{-1}(1-f) \mathbf{S} \boldsymbol{\Sigma}^{-1} \mathbf{S} \Sigma^{-1} \\
& -\mathbf{J}_{T} \otimes \frac{T^{2}}{N}(T(1-f) \mathbf{S}+\mathbf{\Sigma})^{-1}(1-f) \mathbf{S} \boldsymbol{\Sigma}^{-1} \mathbf{S}(T \mathbf{S}+\boldsymbol{\Sigma})^{-1} \mathbf{S} \boldsymbol{\Sigma}^{-1} .
\end{aligned}
$$

Seja $\mathbf{A}=T \mathbf{S}+\boldsymbol{\Sigma}$ e $\mathbf{D}=T(1-f) \mathbf{S}+\boldsymbol{\Sigma}$.

$$
\begin{aligned}
\mathrm{V}^{-1}= & \left(\mathrm{I}_{n} \otimes \mathrm{I}_{T} \otimes \Sigma^{-1}\right)-\left(\mathrm{I}_{n} \otimes \mathrm{J}_{T} \otimes \mathrm{A}^{-1} \mathrm{~S} \Sigma^{-1}\right) \\
& +\left(\mathrm{J}_{n} \otimes \mathrm{J}_{T} \otimes \frac{1}{N} \Sigma^{-1} \mathrm{~S} \Sigma^{-1}\right)-\left(\mathrm{J}_{n} \otimes \mathrm{J}_{T} \otimes \frac{1}{N} T \Sigma^{-1} \mathrm{SA} \mathrm{A}^{-1} \mathrm{~S} \Sigma^{-1}\right) \\
& -\left(\mathrm{J}_{n} \otimes \mathrm{J}_{T} \otimes \frac{1}{N} T \mathrm{D}^{-1}(1-f) \mathrm{S} \Sigma^{-1} \mathrm{~S} \Sigma^{-1}\right) \\
& +\left(\mathrm{J}_{n} \otimes \mathrm{J}_{T} \otimes \frac{1}{N} T^{2} \mathrm{D}^{-1}(1-f) \mathrm{S} \Sigma^{-1} \mathrm{SA} \mathrm{A}^{-1} \mathrm{~S} \Sigma^{-1}\right) \\
= & \left(\mathrm{I}_{n} \otimes \mathrm{I}_{T} \otimes \Sigma^{-1}\right)-\left(\mathrm{I}_{n} \otimes \mathrm{J}_{T} \otimes \mathrm{A}^{-1} \mathrm{~S} \Sigma^{-1}\right) \\
& +\left(\mathrm { J } _ { n } \otimes \mathrm { J } _ { T } \otimes \frac { 1 } { N } \left(\Sigma^{-1}-\Sigma^{-1} T S \mathrm{~A}^{-1}-\mathrm{D}^{-1} T(1-f) \mathrm{S} \Sigma^{-1}\right.\right.
\end{aligned}
$$




$$
\begin{aligned}
& \left.\left.+\mathrm{D}^{-1} T(1-f) \mathrm{S} \Sigma^{-1} T \mathrm{SA}^{-1}\right) \mathrm{~S} \Sigma^{-1}\right) \\
= & \left(\mathrm{I}_{n} \otimes \mathrm{I}_{T} \otimes \Sigma^{-1}\right)-\left(\mathrm{I}_{n} \otimes \mathrm{J}_{T} \otimes \mathrm{A}^{-1} \mathrm{~S} \Sigma^{-1}\right) \\
& +\left(\mathrm { J } _ { n } \otimes \mathrm { J } _ { T } \otimes \frac { 1 } { N } \left(\Sigma^{-1}-\Sigma^{-1}(T \mathrm{~S}+\Sigma-\Sigma) \mathrm{A}^{-1}-\mathrm{D}^{-1}(T(1-f) \mathrm{S}+\Sigma-\Sigma) \Sigma^{-1}\right.\right. \\
& \left.\left.+\mathrm{D}^{-1}(T(1-f) \mathrm{S}+\Sigma-\Sigma) \Sigma^{-1}(T \mathrm{~S}+\Sigma-\Sigma) \mathrm{A}^{-1}\right) \mathrm{~S} \Sigma^{-1}\right) \\
= & \left(\mathrm{I}_{n} \otimes \mathrm{I}_{T} \otimes \Sigma^{-1}\right)-\left(\mathrm{I}_{n} \otimes \mathrm{J}_{T} \otimes \mathrm{A}^{-1} \mathrm{~S} \Sigma^{-1}\right) \\
& +\left(\mathrm { J } _ { n } \otimes \mathrm { J } _ { T } \otimes \frac { 1 } { N } \left(\Sigma^{-1}-\Sigma^{-1}+\mathrm{A}^{-1}-\Sigma^{-1}+\mathrm{D}^{-1}+\Sigma^{-1}-\mathrm{A}^{-1}-\mathrm{D}^{-1}\right.\right. \\
& \left.\left.+\mathrm{D}^{-1} \Sigma \mathrm{A}^{-1}\right) \mathrm{~S} \Sigma^{-1}\right)
\end{aligned}
$$

$\mathrm{V}^{-1}=\left(\mathrm{I}_{n} \otimes \mathrm{I}_{T} \otimes \Sigma^{-1}\right)-\left(\mathrm{I}_{n} \otimes \mathrm{J}_{T} \otimes \mathrm{A}^{-1} \mathrm{~S} \Sigma^{-1}\right)+\frac{1}{N}\left(\mathrm{~J}_{n} \otimes \mathrm{J}_{T} \otimes \mathrm{D}^{-1} \Sigma \mathrm{A}^{-1} \mathrm{~S} \Sigma^{-1}\right)$, ou seja,

$$
\mathrm{V}^{-1}=\left(\mathrm{I}_{n} \otimes \mathrm{I}_{T} \otimes \Sigma^{-1}\right)-\left(\mathrm{I}_{n} \otimes \mathbf{J}_{T} \otimes \mathbf{H}\right)+\left(\mathrm{J}_{n} \otimes \mathbf{J}_{T} \otimes \mathrm{G}\right)
$$

onde

$$
\mathbf{H}=(T \mathbf{S}+\boldsymbol{\Sigma})^{-1} \mathbf{S} \boldsymbol{\Sigma}^{-1} \text { e } \quad \mathbf{G}=\frac{1}{N}(T(1-f) \mathbf{S}+\boldsymbol{\Sigma})^{-1} \boldsymbol{\Sigma}(T \mathbf{S}+\boldsymbol{\Sigma})^{-1} \mathbf{S} \boldsymbol{\Sigma}^{-1} .
$$

C.2. CÁlCULO DA MATriz $\left(\mathrm{X}^{\prime} \mathrm{V}^{-1} \mathrm{X}\right)^{-1}$ E DA MATrIZ Q

Cálculo de $\left(\mathrm{X}^{\prime} \mathrm{V}^{-1} \mathrm{X}\right)^{-1}$

$$
\begin{aligned}
\mathrm{X} & =\mathrm{I}_{n} \otimes \mathrm{I}_{T} \otimes \mathrm{I}_{p} \\
\mathrm{~V}^{-1} & =\left(\mathrm{I}_{n} \otimes \mathrm{I}_{T} \otimes \Sigma^{-1}\right)-\left(\mathrm{I}_{n} \otimes \mathrm{J}_{T} \otimes \mathrm{H}\right)+\left(\mathrm{J}_{n} \otimes \mathrm{J}_{T} \otimes \mathrm{G}\right)
\end{aligned}
$$


H e G dados na seção anterior.

$$
\begin{aligned}
\mathrm{X}^{\prime} \mathrm{V}^{-1} & =\left(\mathbf{1}_{n}^{\prime} \otimes \mathrm{I}_{T} \otimes \Sigma^{\cdots 1}\right)-\left(\mathbf{1}_{n}^{\prime} \otimes \mathbf{J}_{T} \otimes \mathbf{H}\right)+\left(\mathbf{I}_{n}^{\prime} \otimes \mathrm{J}_{T} \otimes n \mathrm{G}\right) \\
\mathbf{X}^{\prime} \mathrm{V}^{-1} \mathrm{X} & =\left(\mathbf{I}_{T} \otimes n \Sigma^{-1}\right)-\left(\mathbf{J}_{T} \otimes n \mathrm{H}\right)+\left(\mathbf{J}_{T} \otimes n^{2} \mathbf{G}\right) \\
& =\left(\mathbf{I}_{T} \otimes n \Sigma^{-1}\right)+\left(\mathbf{J}_{T} \otimes\left(n^{2} \mathbf{G}-n \mathbf{H}\right)\right) .
\end{aligned}
$$

Identificando $\mathrm{B}=n \Sigma^{-1}$ e $\mathrm{C}=n^{2} \mathrm{G}-n \mathrm{H}$ e usando o resultado $\mathrm{A} .3$ do apendice $\mathrm{A}$, temos,

$$
\begin{aligned}
\mathrm{B}^{-1} & =\frac{1}{n} \Sigma \\
T \mathrm{C}+\mathrm{B} & =T n^{2} \mathrm{G}-n T \mathrm{H}+n \Sigma^{-1} \\
\left(\mathrm{X}^{\prime} \mathrm{V}^{-1} \mathrm{X}\right)^{-1} & =\left(\dot{I}_{T} \otimes \frac{1}{n} \Sigma\right)-\left(\mathrm{J}_{T} \otimes \frac{1}{n}\left(T n^{2} \mathrm{G}-n T \mathrm{H}+n \Sigma^{-1}\right)^{-1}\left(n^{2} \mathrm{G}-n \mathbf{H}\right) \Sigma\right)
\end{aligned}
$$

Cálculo da matriz Q

$$
\begin{gathered}
\mathrm{Q}=\mathrm{V}^{-1} \mathrm{X}\left(\mathrm{X}^{\prime} \mathrm{V}^{-1} \mathrm{X}\right)^{-1} \mathrm{X}^{\prime} \mathrm{V}^{-1}+\mathrm{I}_{n T p}-\mathrm{V}^{-1} \\
\left(\mathrm{X}^{\prime} \mathrm{V}^{-1} \mathrm{X}\right)^{-1} \mathrm{X}^{\prime} \mathrm{V}^{-1}=\frac{1}{n}\left(1_{n}^{\prime} \otimes \mathrm{I}_{T} \otimes \mathrm{I}_{p}\right)-\frac{1}{n}\left(\mathbf{1}_{n}^{\prime} \otimes \mathrm{J}_{T} \otimes \Sigma \mathrm{H}\right) \\
+\left(\mathbf{1}_{n}^{\prime} \otimes \mathrm{J}_{T} \otimes \Sigma \mathrm{G}\right)-\frac{1}{n}\left(\mathbf{1}_{n}^{\prime} \otimes \mathrm{J}_{T} \otimes\left(T n^{2} \mathrm{G}-n T \mathrm{H}+n \Sigma^{-1}\right)^{-1}\left(n^{2} \mathrm{G}-n \mathrm{H}\right)\right) \\
+\frac{T}{n}\left(1_{n}^{\prime} \otimes \mathrm{J}_{T} \otimes\left(T n^{2} \mathrm{G}-n T \mathrm{H}+n \Sigma^{-1}\right)^{-1}\left(n^{2} \mathrm{G}-n \mathrm{H}\right) \Sigma \mathrm{H}\right) \\
-T\left(\mathbf{1}_{n}^{\prime} \otimes \mathrm{J}_{T} \otimes\left(T n^{2} \mathrm{G}-n T \mathrm{H}+n \Sigma^{-1}\right)^{-1}\left(n^{2} \mathrm{G}-n \mathrm{H}\right) \Sigma \mathrm{G}\right)
\end{gathered}
$$




$$
\begin{aligned}
& =\frac{1}{n}\left(1_{n}^{\prime} \otimes \mathrm{I}_{T} \otimes \mathrm{I}_{p}\right)+\frac{1}{n}\left(1_{n}^{\prime} \otimes \mathrm{J}_{T} \otimes(-\Sigma \mathrm{H}+n \Sigma \mathrm{G}\right. \\
& -\left(T n \mathrm{G}-T \mathrm{H}+\boldsymbol{\Sigma}^{-1}\right)^{-1}(n \mathrm{G}-\mathbf{H})+T\left(T n \mathrm{G}-T \mathbf{H}+\boldsymbol{\Sigma}^{-1}\right)^{-1}(n \mathbf{G}-\mathbf{H}) \Sigma \mathbf{H} \\
& \left.\left.-T n\left(T n \mathrm{G}-T \mathbf{H}+\Sigma^{-1}\right)^{-1}(n \mathrm{G}-\mathbf{H}) \mathbf{\Sigma} \mathbf{G}\right)\right) \\
& =\frac{1}{n}\left(1_{n}^{\prime} \otimes \mathrm{I}_{T} \otimes \mathrm{I}_{p}\right)+\frac{1}{n}\left(1_{n}^{\prime} \otimes \mathrm{J}_{T} \otimes(-\Sigma \mathrm{H}+n \Sigma \mathrm{G}\right. \\
& -\frac{1}{T}\left(T n \mathrm{G}-T \mathrm{H}+\Sigma^{-1}\right)^{-1}\left(T n \mathrm{G}-T \mathbf{H}+\Sigma^{-1}-\Sigma^{-1}\right) \\
& +\left(T n \mathrm{G}-T \mathrm{H}+\boldsymbol{\Sigma}^{-1}\right)^{-1}\left(T n \mathrm{G}-T \mathrm{H}+\boldsymbol{\Sigma}^{-1}-\boldsymbol{\Sigma}^{-1}\right) \boldsymbol{\Sigma} \mathrm{H} \\
& \left.\left.-n\left(T n \mathbf{G}-T \mathbf{H}+\boldsymbol{\Sigma}^{-1}\right)^{-1}\left(T n \mathrm{G}-T \mathbf{H}+\boldsymbol{\Sigma}^{-1}-\boldsymbol{\Sigma}^{-1}\right) \boldsymbol{\Sigma} \mathrm{G}\right)\right) \\
& =\frac{1}{n}\left(1_{n}^{\prime} \otimes \mathrm{I}_{T} \otimes \mathrm{I}_{p}\right)+\frac{1}{n}\left(1_{n}^{\prime} \otimes \mathrm{J}_{T} \otimes(-\Sigma \mathrm{H}+n \Sigma \mathrm{G}\right. \\
& -\frac{1}{T} \mathrm{I}_{p}+\frac{1}{T}\left(T n \mathrm{G}-T \mathrm{H}+\Sigma^{-1}\right)^{-1} \Sigma^{-1}+\Sigma \mathbf{H} \\
& \left.\left.-\left(T n \mathrm{G}-T \mathrm{H}+\boldsymbol{\Sigma}^{-1}\right)^{-1} \mathbf{H}-n \boldsymbol{\Sigma} \mathbf{G}+\left(T n \mathrm{G}-T \mathbf{H}+\boldsymbol{\Sigma}^{-1}\right)^{-1} n \mathrm{G}\right)\right) \\
& =\frac{1}{n}\left(\mathbf{1}_{n}^{\prime} \otimes \mathbf{I}_{T} \otimes \mathbf{I}_{p}\right)+\frac{1}{n}\left(\mathbf { 1 } _ { n } ^ { \prime } \otimes \mathbf { J } _ { T } \otimes \left(-\frac{1}{T} \mathbf{I}_{p}\right.\right. \\
& \left.\left.+\frac{1}{T}\left(T n \mathbf{G}-T \mathbf{H}+\boldsymbol{\Sigma}^{-1}\right)^{-1}\left(\boldsymbol{\Sigma}^{-1}-T \mathbf{H}+T n \mathbf{G}\right)\right)\right) \\
& =\frac{1}{n}\left(\mathbf{1}_{n}^{\prime} \otimes \mathrm{I}_{T} \otimes \mathrm{I}_{p}\right)
\end{aligned}
$$

$$
\begin{gathered}
\mathrm{V}^{-1} \mathrm{X}\left(\mathrm{X}^{\prime} \mathrm{V}^{-1} \mathrm{X}\right)^{-1} \mathrm{X}^{\prime} \mathrm{V}^{-1}=\frac{1}{n}\left(\mathrm{~J}_{n} \otimes \mathrm{I}_{T} \otimes \Sigma^{-1}\right)-\frac{1}{n}\left(\mathrm{~J}_{n} \otimes \mathrm{J}_{T} \otimes \mathrm{H}\right)+\frac{1}{n}\left(\mathrm{~J}_{n} \otimes \mathrm{J}_{T} \otimes n \mathrm{G}\right) \\
\mathrm{Q}=\frac{1}{n}\left(\mathrm{~J}_{n} \otimes \mathrm{I}_{T} \otimes \Sigma^{-1}\right)-\frac{1}{n}\left(\mathrm{~J}_{n} \otimes \mathrm{J}_{T} \otimes \mathrm{H}\right)+\frac{1}{n}\left(\mathrm{~J}_{n} \otimes \mathrm{J}_{T} \otimes n \mathrm{G}\right)
\end{gathered}
$$


$+\left(\mathrm{I}_{n} \otimes \mathrm{I}_{T} \otimes \mathrm{I}_{p}\right)-\left(\mathrm{I}_{n} \otimes \mathrm{I}_{T} \otimes \Sigma^{-1}\right)+\left(\mathrm{I}_{n} \otimes \mathrm{J}_{T} \otimes \mathrm{H}\right)-\left(\mathrm{J}_{n} \otimes \mathrm{J}_{T} \otimes \mathrm{G}\right)$

$\mathrm{Q}=\left(\mathrm{I}_{n} \otimes \mathrm{I}_{T} \otimes \mathrm{I}_{p}\right)+\left(\frac{1}{n} \mathrm{~J}_{n}-\mathrm{I}_{n}\right) \otimes\left(\mathrm{I}_{T} \otimes \Sigma^{-1}-\mathrm{J}_{T} \otimes \mathrm{H}\right)$

C.3. UMA OBSERVAÇÃO PERDIDA - DEMONSTRAÇÃO DE QUE A EXPRESSÃO (4.10) É IGUAL À EXPRESSÃO (4.11)

Seja

$$
\begin{aligned}
\widetilde{\mathrm{C}}_{. i} & =\mathrm{C}_{\cdot i}=\sum_{t=1}^{T} \mathrm{C}_{t i} \operatorname{para} i=1,2, \ldots, n-1 \\
\widetilde{\mathrm{C}}_{\cdot n} & =\mathrm{C}_{\cdot n}-\mathrm{I}_{p}=\sum_{t=1}^{T} \mathrm{C}_{t n}-\mathrm{I}_{p} \\
\overline{\mathrm{C}}_{t i} & =\mathrm{C}_{t i} \text { para } t=1,2, \ldots, T \text { e } i=1,2, \ldots, n-1 \\
& \quad \text { ou para } t=1,2, \ldots, T-1 \mathrm{e} i=1,2, \ldots, n \\
\overline{\mathrm{C}}_{T n} & =\mathrm{I}_{p} .
\end{aligned}
$$

Note que

$$
\begin{aligned}
& \mathbb{E}\left[( \sum _ { t = 1 } ^ { T } \sum _ { i = 1 } ^ { n } \mathrm { C } _ { t i } \mu _ { t } - \mu _ { T } ) \left(\sum_{t=1}^{T} \sum_{i=1}^{n} \mathrm{C}_{t i} \mu_{t}-\mu_{T}+\sum_{t=1}^{T} \sum_{i=1}^{n} \mathrm{C}_{t i} \mathrm{~b}_{i}\right.\right. \\
& \left.\left.-\mathrm{b}_{n}+\sum_{t=1}^{T} \sum_{i=1}^{n} \mathrm{C}_{t i} \mathrm{e}_{t i}-\mathrm{e}_{T n}\right)^{\prime}\right] \\
= & {\left[\left(\sum_{t=1}^{T} \sum_{i=1}^{n} \mathrm{C}_{t i} \mu_{t}-\boldsymbol{\mu}_{T}\right)\left(\sum_{t=1}^{T} \sum_{i=1}^{n} \mathrm{C}_{t i} \mu_{t}-\mu_{T}\right)\right] } \\
= & \left(\sum_{t=1}^{T} \boldsymbol{\mu}_{t} \sum_{i=1}^{n} \mathrm{C}_{t i}-\mu_{T}\right)\left(\sum_{t=1}^{T} \boldsymbol{\mu}_{t} \sum_{i=1}^{n} \mathrm{C}_{t i}-\mu_{T}\right)^{\prime}=\left(\boldsymbol{\mu}_{T}-\boldsymbol{\mu}_{T}\right)\left(\boldsymbol{\mu}_{T}-\boldsymbol{\mu}_{T}\right)^{\prime}=0
\end{aligned}
$$


Usando a notação e resultado acima,

$$
\begin{aligned}
= & \mathbb{E}\left[\left(\mathrm{l}^{\prime} \sum_{t=1}^{T} \sum_{i=1}^{n} \mathrm{C}_{t i} \mathrm{y}_{t i}-\mathrm{l}^{\prime}\left(\mu_{T}+\mathrm{b}_{n}+\mathrm{e}_{T n}\right)\right)^{2}\right] \\
= & \mathrm{l}^{\prime} \mathbb{E}\left[\left(\sum_{t=1}^{T} \sum_{i=1}^{n} \mathrm{C}_{t i} \mathrm{y}_{t i}-\mu_{T}-\mathrm{b}_{n}-\mathrm{e}_{T n}\right)\left(\sum_{t=1}^{T} \sum_{i=1}^{n} \mathrm{C}_{t i} \mathrm{y}_{t i}-\mu_{T}-\mathrm{b}_{n}-\mathrm{e}_{T n}\right)^{\prime}\right] \mathrm{l} \\
= & \mathrm{l}^{\prime} \mathbb{E}\left[\left(\sum_{t=1}^{T} \sum_{i=1}^{n} \mathrm{C}_{t i}\left(\mu_{t}+\mathrm{b}_{i}+\mathrm{e}_{t i}\right)-\mu_{T}-\mathrm{b}_{n}-\mathrm{e}_{T n}\right)\right)\left(\sum _ { t = 1 } ^ { T } \sum _ { i = 1 } ^ { n } \mathrm { C } _ { t i } \left(\mu_{t}\right.\right. \\
& \left.\left.\left.+\mathrm{b}_{i}+\mathrm{e}_{t i}\right)-\mu_{T}-\mathrm{b}_{n}-\mathrm{e}_{T n}\right)^{\prime}\right] \mathrm{l} \\
= & \mathrm{l}^{\prime} \mathbb{E}\left[\left(\sum_{t=1}^{T} \sum_{i=1}^{n} \mathrm{C}_{t i} \mu_{t}-\mu_{T}+\sum_{t=1}^{T} \sum_{i=1}^{n} \mathrm{C}_{t i} \mathrm{~b}_{i}-\mathrm{b}_{n}+\sum_{t=1}^{T} \sum_{i=1}^{n} \mathrm{C}_{t i} \mathrm{e}_{t i}-\mathrm{e}_{T n}\right)\right. \\
& \left.\cdot\left(\sum_{t=1}^{T} \sum_{i=1}^{n} \mathrm{C}_{t i} \mu_{t}-\mu_{T}+\sum_{t=1}^{T} \sum_{i=1}^{n} \mathrm{C}_{t i} \mathrm{~b}_{i}-\mathrm{b}_{n}+\sum_{t=1}^{T} \sum_{i=1}^{n} \mathrm{C}_{t i} \mathrm{e}_{t i}-\mathrm{e}_{T n}\right)^{\prime}\right] \mathrm{l} \\
= & \mathrm{l}^{\prime} \mathbb{E}\left[\left(\sum_{t=1}^{T} \sum_{i=1}^{n} \mathrm{C}_{t i} \mathrm{~b}_{i}-\mathrm{b}_{n}\right)\left(\sum_{t=1}^{T} \sum_{i=1}^{n} \mathrm{C}_{t i} \mathrm{~b}_{i}-\mathrm{b}_{n}\right)^{\prime}\right. \\
& \left.+\left(\sum_{t=1}^{T} \sum_{i=1}^{n} \mathrm{C}_{t i} \mathrm{e}_{t i}-\mathrm{e}_{T n}\right)\left(\sum_{t=1}^{T} \sum_{i=1}^{n} \mathrm{C}_{t i} \mathrm{e}_{t i}-\mathrm{e}_{T n}\right)^{\prime}\right] \mathrm{l} \\
= & \mathrm{l}^{\prime} \mathbb{E}\left[\left(\sum_{i=1}^{n} \mathrm{C}_{. i} \mathrm{~b}_{i}-\mathrm{b}_{n}\right)\left(\sum_{i=1}^{n} \mathrm{C}_{i} \mathrm{~b}_{i}-\mathrm{b}_{n}\right)^{\prime}\right. \\
& \left.+\left(\sum_{t=1}^{T} \sum_{i=1}^{n} \mathrm{C}_{t i} \mathrm{e}_{t i}-\mathrm{e}_{T n}\right)\left(\sum_{t=1}^{T} \sum_{i=1}^{n} \mathrm{C}_{t i} \mathrm{e}_{t i}-\mathrm{e}_{T n}\right)^{\prime}\right] \mathrm{l} \\
= & \mathrm{l}^{\prime} \mathbb{E}\left[\left(\sum_{i=1}^{n} \tilde{\mathrm{C}}_{. i} \mathrm{~b}_{i}\right)\left(\sum_{i=1}^{n} \tilde{\mathrm{C}}_{i} \mathrm{~b}_{i}\right)^{\prime}+\left(\sum_{t=1}^{T} \sum_{i=1}^{n} \overline{\mathrm{C}}_{t i} \mathrm{e}_{t i}\right)^{\prime}\left(\sum_{t=1}^{T} \sum_{i=1}^{n} \overline{\mathrm{C}}_{t i} \mathrm{e}_{t i}\right)^{\prime}\right] \mathrm{l}
\end{aligned}
$$

Mas

$$
\begin{aligned}
& \mathbb{E}\left[\left(\sum_{i=1}^{n} \tilde{\mathrm{C}}_{\cdot i} \mathrm{~b}_{i}\right)\left(\sum_{i=1}^{n} \tilde{\mathrm{C}}_{\cdot i} \mathrm{~b}_{i}\right)^{\prime}\right]=\mathbb{E}\left[\sum_{i=1}^{n} \sum_{j=1}^{n} \tilde{\mathrm{C}}_{. i} \mathrm{~b}_{i} \mathrm{~b}_{j}^{\prime} \tilde{\mathrm{C}}_{\cdot j}^{\prime}\right] \\
= & \sum_{i=1}^{n} \sum_{j=1}^{n} \widetilde{\mathrm{C}}_{\cdot i} \mathbb{E}\left(\mathrm{b}_{i} \mathrm{~b}_{j}^{\prime}\right) \widetilde{\mathrm{C}}_{\cdot j}^{\prime} \\
= & \sum_{i=1}^{n} \tilde{\mathrm{C}}_{\cdot i} \mathbb{E}\left(\mathrm{b}_{i} \mathrm{~b}_{i}^{\prime}\right) \tilde{\mathrm{C}}_{\cdot i}^{\prime}+\sum_{\substack{i=1 \\
j \neq i}}^{n} \sum_{\substack{j=1 \\
n}}^{n} \tilde{\mathrm{C}}_{\cdot i} \mathbb{E}\left(\mathrm{b}_{i} \mathrm{~b}_{j}^{\prime}\right) \tilde{\mathrm{C}}_{\cdot j}^{\prime}
\end{aligned}
$$




$$
\begin{aligned}
& =\sum_{i=1}^{n} \widetilde{\mathrm{C}}_{. i} \frac{(N-1)}{N} \mathrm{~S} \tilde{\mathrm{C}}_{\cdot i}^{\prime}-\sum_{i=1}^{n} \sum_{\substack{j=1 \\
j \neq i}}^{n} \tilde{\mathrm{C}}_{\cdot i} \frac{1}{N} \mathrm{~S} \tilde{\mathrm{C}}_{\cdot j}^{\prime} \\
& =\frac{(N-1)}{N} \sum_{i=1}^{n} \tilde{\mathrm{C}}_{\cdot i} \mathrm{~S} \tilde{\mathrm{C}}_{\cdot i}^{\prime}-\frac{1}{N} \sum_{i=1}^{n} \sum_{j=1}^{n} \widetilde{\mathrm{C}}_{\cdot i} \mathrm{~S} \widetilde{\mathrm{C}}_{\cdot j}^{\prime}+\frac{1}{N} \sum_{i=1}^{n} \tilde{\mathrm{C}}_{. i} \mathrm{~S} \tilde{\mathrm{C}}_{\cdot i}^{\prime} \\
& =\sum_{i=1}^{n} \tilde{\mathrm{C}}_{. i} \mathrm{~S} \widetilde{\mathrm{C}}_{\cdot i}^{\prime}-\frac{1}{N}\left(\sum_{i=1}^{n} \tilde{\mathrm{C}}_{\cdot i}\right) \mathrm{S}\left(\sum_{i=1}^{n} \tilde{\mathrm{C}}_{\cdot i}\right)^{\prime}
\end{aligned}
$$

e

$$
\begin{aligned}
& \mathbb{E}\left[\left(\sum_{t=1}^{T} \sum_{i=1}^{n} \overline{\mathrm{C}}_{t i} \mathrm{e}_{t i}\right)\left(\sum_{t=1}^{T} \sum_{i=1}^{n} \overline{\mathrm{C}}_{t i} \mathrm{e}_{t i}\right)^{\prime}\right] \\
= & \mathbb{E}\left[\sum_{i=1}^{n} \sum_{t=1}^{T} \overline{\mathrm{C}}_{t i} \mathrm{e}_{t i} \mathrm{e}_{t i}^{\prime} \overline{\mathrm{C}}_{t i}^{\prime}\right]=\sum_{i=1}^{n} \sum_{t=1}^{T} \overline{\mathrm{C}}_{t i} \Sigma \overline{\mathrm{C}}_{t i}^{\prime}
\end{aligned}
$$

e portanto

$$
\begin{aligned}
(4.10)= & \mathrm{l}^{\prime}\left[\sum_{i=1}^{n} \tilde{\mathrm{C}}_{. i} \mathrm{~S} \tilde{\mathrm{C}}_{\cdot i}-\frac{1}{N}\left(\sum_{i=1}^{n} \tilde{\mathrm{C}}_{\cdot i}\right) \mathrm{S}\left(\sum_{i=1}^{n} \tilde{\mathrm{C}}_{\cdot i}\right)^{\prime}\right. \\
& \left.+\sum_{i=1}^{n} \sum_{t=1}^{T} \overline{\mathrm{C}}_{t i} \Sigma \overline{\mathrm{C}}_{t i}^{\prime}\right] \mathrm{l}=(4.11)
\end{aligned}
$$

C.4. VERIFICAÇÃO DA SOLUÇÃO DO SISTEMA DE EQUAÇÕES

(1) Para qualquer $j=1,2, \ldots, n-1$

$$
\begin{aligned}
& 2 \mathrm{ll}^{\prime} \sum_{t=1}^{T} \mathrm{C}_{t j} \mathrm{~S}+2 \mathrm{ll}^{\prime} \mathrm{C}_{T j} \Sigma+\Lambda_{T}^{\prime} \\
= & 2 \mathrm{ll}^{\prime}\left(-\frac{(T-1)}{(n-1)} \mathrm{S}(\mathrm{S}(T-1)+\Sigma)^{-1}+\frac{1}{(n-1)} \mathrm{I}_{p}\right) \mathrm{S}+\frac{2 \mathrm{ll}^{\prime}}{(n-1)} \Sigma \\
& -\frac{2 \mathrm{ll}^{\prime}}{(n-1)}\left(-(T-1) \mathrm{S}(\mathrm{S}(T-1)+\Sigma)^{-1} \mathrm{~S}+\mathrm{S}+\Sigma\right) \\
= & \frac{2 \mathrm{ll}^{\prime}}{(n-1)}\left(-(T-1) \mathrm{S}(\mathrm{S}(T-1)+\Sigma)^{-1} \mathrm{~S}+\mathrm{S}+\boldsymbol{\Sigma}\right. \\
& \left.+(T-1) \mathrm{S}(\mathrm{S}(T-1)+\Sigma)^{-1} \mathrm{~S}-\mathrm{S}-\Sigma\right)=\mathbf{0}
\end{aligned}
$$


(2) Para qualquer $v=1,2, \ldots, T-1$

$$
\begin{aligned}
& 2 \mathrm{ll}^{\prime}\left(\sum_{t=1}^{T} \mathrm{C}_{t n}\right) \mathrm{S}-2 \mathrm{ll}^{\prime} \mathrm{S}+2 \mathrm{ll}^{\prime} \mathrm{C}_{v n} \Sigma+\Lambda_{v}^{\prime} \\
= & 2 \mathrm{ll}^{\prime}\left((T-1) \mathrm{S}(\mathrm{S}(T-1)+\Sigma)^{-1} \mathrm{~S}-\mathrm{S}+\mathrm{S}(\mathrm{S}(T-1)+\Sigma)^{-1} \Sigma\right) \\
= & 2 \mathrm{ll}^{\prime}\left(\mathrm{S}(\mathrm{S}(T-1)+\Sigma)^{-1}((T-1) \mathrm{S}+\Sigma)-\mathrm{S}\right)=0
\end{aligned}
$$

(3) Para qualquer $j=1,2, \ldots, n-1$ e qualquer $v=1,2, \ldots, T-1$

$$
\begin{aligned}
& 2 \mathrm{ll}^{\prime}\left(\sum_{t=1}^{T} \mathrm{C}_{t j}\right) \mathrm{S}+2 \mathrm{ll}^{\prime} \mathrm{C}_{v j} \Sigma+\Lambda_{v}^{\prime} \\
= & 2 \mathrm{ll}^{\prime}\left(-\frac{(T-1)}{(n-1)} \mathrm{S}(\mathrm{S}(T-1)+\Sigma)^{-1} \mathrm{~S}+\frac{1}{(n-1)} \mathrm{S}-\frac{1}{(n-1)} \mathrm{S}(\mathrm{S}(T-1)+\Sigma)^{-1} \Sigma\right) \\
= & \frac{2 \mathrm{ll}^{\prime}}{(n-1)}\left(-\mathrm{S}(\mathrm{S}(T-1)+\Sigma)^{-1}((T-1) \mathrm{S}+\Sigma)+\mathrm{S}\right)=0
\end{aligned}
$$

(4) Para qualquer $v=1,2, \ldots, T-1$

$$
\left(\sum_{i=1}^{n} \mathrm{C}_{v i}\right)^{\prime}=\left(-(\mathrm{S}(T-1)+\Sigma)^{-1} \mathrm{~S}+(\mathrm{S}(T-1)+\Sigma)^{-1} \mathrm{~S}\right)=0
$$

(5) $\left(\sum_{i=1}^{n-1} \mathrm{C}_{T i}-\mathrm{I}_{p}\right)^{\prime}=\frac{(n-1)}{(n-1)} \mathrm{I}_{p}-\mathrm{I}_{p}=0$

(6) $\mathrm{C}_{T n}^{\prime}=0$ 


\section{APÊNDICE D}

\section{ESTIMAÇÃO DA VARIÂNCIA}

D.1. ESPERANÇA DOS QUADRADOS MÉDIOS

Seja $B=S S_{\text {ind }}=\frac{1}{T} \mathrm{y}^{\prime}\left(\mathrm{I}_{n T}-\mathrm{X}\left(\mathrm{X}^{\prime} \mathbf{X}\right)^{-1} \mathrm{X}^{\prime}\right) \mathrm{ZZ}^{\prime} \mathrm{y}$.

$\operatorname{Mas} \mathbf{X}\left(\mathbf{X}^{\prime} \mathbf{X}\right)^{-1} \mathbf{X}^{\prime}=\frac{1}{n}\left(\mathbf{J}_{n} \otimes \mathbf{I}_{T}\right)$ e $\mathbf{Z}=\left(\mathrm{I}_{n} \otimes \mathbf{1}_{T}\right)$ e então

$$
\begin{aligned}
S S_{\text {ind }} & =\frac{1}{T}\left(\mathbf{y}^{\prime}\left(\left(\mathbf{I}_{n} \otimes \mathbf{J}_{T}\right)-\frac{1}{n}\left(\mathbf{J}_{n} \otimes \mathbf{J}_{T}\right)\right) \mathrm{y}\right) \\
\mathbf{V} & =\operatorname{Cov}(\mathbf{y})=\left(\sigma^{2} \mathbf{I}_{n T}+S^{2}\left(\mathbf{I}_{n} \otimes \mathbf{J}_{T}\right)-\frac{S^{2}}{N} \mathbf{J}_{n T}\right)
\end{aligned}
$$

e

$$
\boldsymbol{\mu}=\mathbb{E}(\mathrm{y})
$$

Considerando o fato que 


$$
\mathbb{E}\left(\mathrm{y}^{\prime} \mathrm{Cy}\right)=\operatorname{tr}(\mathrm{CV})+\mu^{\prime} \mathrm{C} \mu
$$

(resultado A.2 do Apêndice A) e que $\mu^{\prime} \mathrm{C} \mu=0$, temos que

$$
\begin{aligned}
\mathbb{E}\left(S S_{\text {ind }}\right)= & \frac{1}{T} \operatorname{tr}\left[( ( \mathbf { I } _ { n } \otimes \mathbf { J } _ { T } ) - \frac { 1 } { n } ( \mathbf { J } _ { n } \otimes \mathbf { J } _ { T } ) ) \left(\sigma^{2}\left(\mathbf{I}_{n} \otimes \mathbf{I}_{T}\right)\right.\right. \\
& \left.\left.+S^{2}\left(\mathbf{I}_{n} \otimes \mathbf{J}_{T}\right)-\frac{S^{2}}{N}\left(\mathbf{J}_{n} \otimes \mathbf{J}_{T}\right)\right)\right] \\
= & \frac{1}{T}\left[\sigma^{2} n T+S^{2} n T^{2}-\frac{S^{2} n T^{2}}{N}-\frac{\sigma^{2} n T}{n}-\frac{S^{2} n T^{2}}{n}+\frac{S^{2} n^{2} T^{2}}{n N}\right] \\
= & (n-1)\left(T S^{2}+\sigma^{2}\right) .
\end{aligned}
$$

Seja

$$
\begin{aligned}
A=\mathrm{y}^{\prime}\left(\mathrm{I}_{n T}-\mathrm{X}\left(\mathrm{X}^{\prime} \mathrm{X}\right)^{-1} \mathrm{X}^{\prime}\right) \mathrm{y}=\mathrm{y}^{\prime}\left(\mathbf{I}_{n T}-\frac{1}{n}\left(\mathbf{J}_{n} \otimes \mathrm{I}_{T}\right)\right) \mathrm{y} . \\
\mathbb{E}(A)=\quad \operatorname{tr}\left[( ( \mathrm { I } _ { n } \otimes \mathrm { I } _ { T } ) - \frac { 1 } { n } ( \mathrm { J } _ { n } \otimes \mathrm { I } _ { T } ) ) \left(\sigma^{2}\left(\mathrm{I}_{n} \otimes \mathbf{I}_{T}\right)\right.\right. \\
\left.\left.\quad+S^{2}\left(\mathrm{I}_{n} \otimes \mathrm{J}_{T}\right)-\frac{S^{2}}{N}\left(\mathrm{~J}_{n} \otimes \mathrm{J}_{T}\right)\right)\right] \\
=\sigma^{2} n T+S^{2} n T-\frac{S^{2} n T}{N}-\frac{\sigma^{2} n T}{n}-\frac{S^{2} n T}{n}+\frac{S^{2} n^{2} T}{n N} \\
=\sigma^{2} T(n-1)+S^{2} T(n-1)=T(n-1)\left(\sigma^{2}+S^{2}\right)
\end{aligned}
$$

Assim,

$$
\begin{aligned}
\mathbb{E}\left(S S_{\mathrm{err}}\right) & =\mathbb{E}(A-B)=T(n-1)\left(\sigma^{2}+S^{2}\right)-(n-1)\left(T S^{2}+\sigma^{2}\right) \\
& =(n-1)(T-1) \sigma^{2}
\end{aligned}
$$

O cálculo de $\mathbb{E}\left(S S_{\mathrm{tem}}\right)$ é análogo ao cálculo de $\mathbb{E}\left(S S_{\text {ind }}\right)$. 


\section{D.2. OBTENÇÃO DO REML}

(1) Obtenção de $\hat{b}_{i}$

$$
\begin{aligned}
& \mathrm{y}=\mathrm{X} \alpha+\mathrm{Zb}+\mathrm{e} \\
& \operatorname{Var}(\mathrm{b})=\mathrm{G} \sigma^{2}, \quad \operatorname{Var}(\mathrm{e})=\sigma^{2} \mathrm{I}_{n T} .
\end{aligned}
$$

De (2.21) do capítulo 2, com y conhecido, temos

$$
\widehat{\mathrm{b}}=\mathrm{G}\left(\mathrm{I}_{n}-\mathrm{Z}^{\prime} \mathrm{W}^{-1} \mathrm{ZG}\right) \mathrm{Z}^{\prime}\left(\mathrm{I}_{n T}-\mathrm{X}\left(\mathrm{X}^{\prime} \mathrm{W}^{-1} \mathrm{X}\right)^{-1} \mathrm{X}^{\prime} \mathrm{W}^{-1}\right) \mathrm{y}
$$

com

$$
\begin{aligned}
& \mathrm{G}=K \mathrm{I}_{n}-\frac{K}{N} \mathrm{~J}_{n}, \quad \mathrm{Z}=\mathrm{I}_{n} \otimes \mathbf{I}_{T} \\
& \mathrm{X}\left(\mathrm{X}^{\prime} \mathrm{W}^{-1} \mathrm{X}\right)^{-1} \mathrm{X}^{\prime} \mathrm{W}^{-1}=\frac{1}{n}\left(\mathrm{~J}_{n} \otimes \mathbf{I}_{T}\right) \\
& \mathrm{W}^{-1}=\left(\mathbf{I}_{n} \otimes \mathbf{I}_{T}\right)-a\left(\mathbf{I}_{n} \otimes \mathbf{J}_{T}\right)+b\left(\mathbf{J}_{n} \otimes \mathbf{J}_{T}\right)
\end{aligned}
$$

onde $a=\frac{K}{T K+1}$ e $b=\frac{K}{N(T K+1)(T K(1-f)+1)}$. Assim,

$$
\mathrm{Z}^{\prime} \mathrm{W}^{-1} \mathrm{Z}=c \mathrm{I}_{n}+d \mathbf{J}_{n}
$$

onde $c=\frac{T}{T K+1}, d=\frac{T K}{N(T K+1)(T K(1-f)+1)}$,

$$
\begin{aligned}
& \mathbf{I}_{n}-\mathbf{Z}^{\prime} \mathbf{W}^{-1} \mathbf{Z G}=(1-c K) \mathbf{I}_{n}+\left(d K(1-f)-\frac{c K}{N}\right) \mathbf{J}_{n} \\
& \cdot \mathbf{G}\left(\mathbf{I}_{n}-\mathbf{Z}^{\prime} \mathbf{W}^{-1} \mathbf{Z G}\right) \mathbf{Z}^{\prime}\left(\mathbf{I}_{n T}-\mathbf{X}\left(\mathbf{X}^{\prime} \mathbf{W}^{-1} \mathbf{X}\right)^{-1} \mathbf{X}^{\prime} \mathbf{W}^{-1}\right) \\
& =g\left(\mathbf{I}_{n} \otimes \mathbf{1}_{T}^{\prime}\right)+h\left(\mathbf{J}_{n} \otimes \mathbf{1}_{T}^{\prime}\right)
\end{aligned}
$$

onde

$$
g=K(1-c K)=\frac{K}{T K+1}
$$




$$
\begin{aligned}
h= & K\left(d K(1-f)-\frac{c K}{N}\right)-\frac{K^{2}}{N}(1-c K)-K^{2} f\left(d K(1-f)-\frac{c K}{N}\right) \\
& -\frac{K}{n}(1-c K)-K\left(d K(1-f)-\frac{c K}{N}\right)+\frac{K^{2}}{N}(1-c K)+K^{2} f\left(d K(1-f)-\frac{c K}{N}\right) \\
= & -\frac{K}{n(T K+1)} .
\end{aligned}
$$

E então, para $i=1,2, \ldots, n$,

$$
\begin{aligned}
\hat{b}_{i} & =\frac{(n-1) K}{n(T K+1)} \sum_{t=1}^{T} y_{t i}-\frac{K}{n(T K+1)} \sum_{\substack { t=1 \\
\begin{subarray}{c}{j=1 \\
j \neq i{ t = 1 \\
\begin{subarray} { c } { j = 1 \\
j \neq i } }\end{subarray}}^{N} y_{t j} \\
& =\frac{T S^{2}}{T S^{2}+\sigma^{2}}\left(\bar{y}_{. i}-\bar{y}_{. .}\right)
\end{aligned}
$$

(2) Cálculo de $\mathbb{E}\left(\hat{b}_{i}^{2}\right)$

Do apêndice D.1 temos que

$$
\mathbb{E}\left(S S_{\mathrm{ind}}\right)=\mathbb{E}\left(\sum_{i=1}^{n} \sum_{t=1}^{T}\left(\bar{y}_{\cdot i}-\bar{y}_{. .}\right)^{2}\right)=(n-1)\left(T S^{2}+\sigma^{2}\right)
$$

ou

$$
T \sum_{i=1}^{n} \mathbb{E}\left(\frac{T S^{2}}{T S^{2}+\sigma^{2}}\left(\bar{y}_{\cdot i}-\bar{y}_{. .}\right)\right)^{2}=(n-1) \frac{T^{2}\left(S^{2}\right)^{2}}{\left(T S^{2}+\sigma^{2}\right)^{2}}\left(T S^{2}+\sigma^{2}\right)
$$

o que implica em

$$
\sum_{i=1}^{n} \mathbb{E}\left(\widehat{b}_{i}^{2}\right)=\frac{T(n-1)\left(S^{2}\right)^{2}}{T S^{2}+\sigma^{2}}
$$

(3) Obtenção de $\hat{e}_{t i}$

Podemos escrever o modelo como

$$
y_{t i}=\mu_{t}+b_{i}+e_{t i} .
$$

O estimador de $\mu_{t}$ é $\hat{\mu}_{t}=\bar{y}_{t}$. e então

$$
\widehat{e}_{t i}=\left(y_{t i}-\bar{y}_{t}\right)-\frac{T S^{2}}{T S^{2}+\sigma^{2}}\left(\bar{y}_{\cdot i}-\bar{y}_{. .}\right) .
$$


(4) Cálculo de $\mathbb{E}\left(\hat{e}_{t i}^{2}\right)$

$$
\begin{aligned}
\hat{e}_{t i}^{2}= & \left(y_{t i}-\bar{y}_{t .}\right)^{2}+\frac{T^{2}\left(S^{2}\right)^{2}}{\left(T S^{2}+\sigma^{2}\right)^{2}}\left(\bar{y}_{\cdot i}-\bar{y}_{. .}\right)^{2} \\
& -\frac{2 T S^{2}}{T S^{2}+\sigma^{2}}\left(y_{t i}-\bar{y}_{t .}\right)\left(\bar{y}_{\cdot i}-\bar{y}_{. .}\right) .
\end{aligned}
$$

Usando a mesma notação anterior com

$$
A=\sum_{i=1}^{n} \sum_{t=1}^{T}\left(y_{t i}-\bar{y}_{t .}\right)^{2} \quad \text { e } \quad B=\sum_{i=1}^{n} \sum_{t=1}^{T}\left(\bar{y}_{. i}-\bar{y}_{. .}\right)^{2}
$$

e verificando que

$$
\sum_{i=1}^{n} \sum_{t=1}^{T}\left(y_{t i}-\bar{y}_{i}\right)\left(\bar{y}_{\cdot i}-\bar{y}_{. .}\right)=T \sum_{i=1}^{n}\left(\bar{y}_{\cdot i}-\bar{y}_{. .}\right)^{2}=B
$$

temos que

$$
\sum_{i=1}^{n} \sum_{t=1}^{T} \hat{e}_{t i}^{2}=A+\frac{T^{2}\left(S^{2}\right)^{2}}{\left(T S^{2}+\sigma^{2}\right)^{2}} B-\frac{2 T S^{2}}{T S^{2}+\sigma^{2}} B
$$

e

$$
\begin{aligned}
& \mathbb{E}\left(\sum_{i=1}^{n} \sum_{t=1}^{T} \hat{e}_{t i}^{2}\right)=T(n-1)\left(S^{2}+\sigma^{2}\right)+\frac{T^{2}\left(S^{2}\right)^{2}}{\left(T S^{2}+\sigma^{2}\right)^{2}}(n-1)\left(T S^{2}+\sigma^{2}\right) \\
& -\frac{2 T S^{2}}{T S^{2}+\sigma^{2}}(n-1)\left(T S^{2}+\sigma^{2}\right)=\frac{T(n-1) \sigma^{2}}{\left(T S^{2}+\sigma^{2}\right)}\left((T-1) S^{2}+\sigma^{2}\right)
\end{aligned}
$$

D.3. VARIÂNCIAS DE $\widehat{S}_{A}^{2}, \widehat{\sigma}_{A}^{2}$ E COVARIÂNCIA ENTRE $\widehat{S}_{A}^{2}$ e $\widehat{\sigma}_{A}^{2}$

A obtenção de $\operatorname{Var}\left(\widehat{S}_{A}^{2}\right), \operatorname{Var}\left(\widehat{\sigma}_{A}^{2}\right)$ e $\operatorname{Cov}\left(\widehat{S}_{A}^{2}, \widehat{\sigma}_{A}^{2}\right)$ foi conseguida através do cálculo direto e levou aos mesmos resultados obtidos pela aplicação das fórmulas dadas por Tukey (1956b), que consiste no seguinte. 
Considere os cumulantes de $2^{\underline{a}}$ e $4^{\underline{a}}$ ordem da distribuição normal, com média zero e variância $\sigma^{2}$ (ver Stuart/Ord (1987))

$$
K_{2}=\sigma^{2}, K_{4}=0
$$

Considere também, as estatísticas $k$, utilizadas para a obtenção dos cumulantes de distribuições amostrais, ou seja, para $x_{i}, i=1,2, \ldots, N$, ou seja,

$$
\begin{aligned}
\langle a b\rangle=\frac{1}{N(N-1)} \sum_{\substack{i \\
j \\
j}}^{N} x_{i}^{a} x_{j}^{b}, \\
i \neq j \\
\langle a\rangle=\frac{1}{N} \sum_{i=1}^{n} x_{i}^{a} .
\end{aligned}
$$

As estatísticas $k$ são dadas por

$$
\begin{aligned}
& k_{2}=\langle 2\rangle-\langle 11\rangle \\
& k_{22}=\langle 22\rangle-2\langle 112\rangle+\langle 1111\rangle \\
& k_{4}=\langle 4\rangle-3\langle 22\rangle-4\langle 13\rangle+12\langle 112\rangle-6\langle 1111\rangle .
\end{aligned}
$$

Segundo Tuckey (1956b), para o modelo

$$
y_{t i}=\mu+\alpha_{t}+b_{i}+e_{t i}, \quad i=1,2, \ldots, n ; t=1,2, \ldots, T,
$$

com cumulantes de $b_{i}$ dados por $k_{a}, k_{a b}$ e cumulantes de $e_{t i}$ dados por $\kappa_{a}, \kappa_{a b}$, a variância do estimador da componente da variância correspondente ao efeito aleatório é

$$
\begin{aligned}
\operatorname{Var}\left(\hat{S}_{A}^{2}\right)= & \left(\frac{1}{n}-\frac{1}{N}\right) k_{4}+\left(\frac{2}{n-1}-\frac{2}{N-1}\right) k_{22}+\frac{4}{T(n-1)} k_{2} \kappa_{2} \\
& +\frac{2}{T(n-1)(T-1)} \kappa_{22},
\end{aligned}
$$


a variância do estimador da componente da variância correspondente ao erro é

$$
\operatorname{Var}\left(\hat{\sigma}_{A}^{2}\right)=\left(\frac{1}{n T}\right) \kappa_{4}+\left(\frac{2}{(n-1)(T-1)}\right) \kappa_{22}
$$

e a covariância entre eles é

$$
\operatorname{Cov}\left(\widehat{S}_{A}^{2}, \widehat{\sigma}_{A}^{2}\right)=-\frac{2}{T(n-1)(T-1)} \kappa_{22} .
$$

Assim,

$$
\begin{aligned}
& k_{2}=\frac{1}{N} \sum_{i=1}^{N} b_{i}^{2}-\frac{1}{N(N-1)} \sum_{\substack{i=1 \\
i \neq j}}^{N} \sum_{\substack{j \neq 1 \\
N}}^{N} b_{i} b_{j}=\frac{(N-1)}{N} S^{2}+\frac{S^{2}}{N}=S^{2} \\
& k_{22}=\frac{1}{N(N-1)} \sum_{i=1}^{N} \sum_{j=1}^{N} b_{i}^{2} b_{j}^{2}-\frac{2}{N(N-1)(N-2)} \sum_{i=1}^{N} \sum_{\substack{j=1 \\
i \neq j \\
i \neq j \\
i \neq k \\
j \neq k}}^{N} \sum_{k=1}^{N} b_{i} b_{j} b_{k}^{2} \\
& +\frac{1}{N(N-1)(N-2)(N-3)} \sum_{\substack{i=1 \\
i \neq j, \quad}}^{N} \sum_{\substack{j=1 \\
i \neq 1}}^{N} \sum_{\substack{k=1 \\
i \neq k}}^{N} \sum_{\substack{l=1 \\
j}}^{N} b_{i} b_{j} b_{k} b_{l} \\
& i \neq l, \quad j \neq k \\
& j \neq l, \quad k \neq l \\
& =\frac{(N-1)}{N}\left(S^{2}\right)^{2}-\frac{S^{4}}{N}+\frac{2(N-1)}{N(N-2)}\left(S^{2}\right)^{2}-\frac{4}{N(N-2)} S^{4} \\
& -\frac{6}{N(N-2)(N-3)} S^{4}+\frac{3(N-1)}{N(N-2)(N-3)}\left(S^{2}\right)^{2} \\
& k_{4}=\frac{1}{N} \sum_{i=1}^{N} b_{i}^{4}-\frac{3}{N(N-1)} \sum_{\substack{i=1 \\
i \neq j}}^{N} \sum_{j=1}^{N} b_{i}^{2} b_{j}^{2}-\frac{4}{N(N-1)} \sum_{i=1}^{N} \sum_{\substack{j \neq 1 \\
i \neq j}}^{N} b_{i} b_{j}^{3}
\end{aligned}
$$




$$
\begin{aligned}
& +\frac{12}{N(N-1)(N-2)} \sum_{i=1}^{N} \sum_{\substack{j=1 \\
i \neq j}}^{N} \sum_{k=1}^{N} b_{i} b_{j} b_{k}^{2} \\
& i \neq k \\
& j \neq k \\
& -\frac{6}{N(N-1)(N-2)(N-3)} \sum_{\substack{i=1 \\
i \neq j, \quad}}^{N} \sum_{\substack{j=1 \\
i \neq 1}}^{N} \sum_{\substack{k=1 \\
i \neq k}}^{N} \sum_{\substack{i=1 \\
i}}^{N} b_{i} b_{j} b_{k} b_{l} \\
& i \neq l, \quad j \neq k \\
& j \neq l, \quad k \neq l \\
& =\frac{(N-1)}{N} S^{4}-\frac{3(N-1)}{N}\left(S^{2}\right)^{2}+\frac{3 S^{4}}{N}+\frac{4 S^{4}}{N}-\frac{12(N-1)}{N(N-2)}\left(S^{2}\right)^{2} \\
& +\frac{24 S^{4}}{N(N-2)}+\frac{36}{N(N-2)(N-3)} S^{4}-\frac{18(N-1)}{N(N-2)(N-3)}\left(S^{2}\right)^{2}
\end{aligned}
$$

$\kappa_{22}=\sigma^{4}$ e portanto,

$$
\begin{aligned}
\operatorname{Var}\left(\widehat{S}_{A}^{2}\right)= & \frac{N-n}{N n} k_{4}+\frac{2(N-n)}{(n-1)(N-1)} k_{22}+\frac{4}{T(n-1)} S^{2} \sigma^{2}+\frac{2}{T(n-1)(T-1)} \sigma^{4} \\
= & \frac{(N-n)[n(N-1)-(N+1)]}{n(n-1)(N-2)(N-3)} S^{4}+\frac{(N-n)\left(-N^{2} n+3 N^{2}-6 N+3 n+3\right)}{N n(n-1)(N-2)(N-3)}\left(S^{2}\right)^{2} \\
& +\frac{4}{T(n-1)} S^{2} \sigma^{2}+\frac{2}{T(n-1)(T-1)} \sigma^{4},
\end{aligned}
$$$$
\operatorname{Var}\left(\widehat{\sigma}_{A}^{2}\right)=\frac{2}{(n-1)(T-1)} \sigma^{4}
$$

e

$$
\operatorname{Cov}\left(\widehat{S}_{A}^{2}, \hat{\sigma}_{A}^{2}\right)=-\frac{2}{T(n-1)(T-1)} \sigma^{4}
$$




\section{REFERÊNCIAS}

[1] Airy, G.B. (1861). On the Algebraical and Numerical Theory of Error of Observations and the Combinations of Observations. London: MacMillan.

[2] Albieri, S. (1989). A Ausência de Resposta em Pesquisas: uma Aplicação de Métodos de Imputação. Dissertação de Mestrado, IMPA-RJ ( *).

[3] Ali, M.A. and Abu-Salih, M.S. (1988). On estimation of missing observations in linear regression models. Sankhya $B, 50,404-411(*)$.

[4] Allan, F.E. and Wishart, J. (1930). A method of estimating yield of missing plot in field experimental work. Journal of Agricultural Science, 20,399-406 (*).

[5] Anderson, A.B.; Basilevsky, A. and Hum, D. (1983). Missing Data. Ch.12 in Rossy, P.H.; Writght, J.D.: Anderson, A. (eds). Handbook of Survey Research, Academic Press, 415-494.

[6] Anderson, R.L. and Bancroft, T.A. (1952). Statistical Theory in Research. New York: McGraw-Hill. 
[7] Bannister, J. (1980). Use and abuse of census editing and imputation (with discussion). The Asian and Pacific Census Forum 6, East-West Population Institute, Honolulu (*).

[8] Bartlett, M.S. (1937). Some examples of statistical methods of research in agriculture and applied botany. Journal of the Royal Statistical Society - B , 4, 137-170 $(*)$.

[9] Basilevsky, A.; Sabourin, D.; Hum, D. and Anderson, A. (1985). Missing data estimators in the general linear model: an evaluation of simulated data as an experimental design. Communications in Statistics B , 14, 371-394.

[10] Bennett, C.A. and Franklin, N.L. (1954). Statistical Analysis in Chemistry and the Chemical Industry, Wiley, NY, $724 \mathrm{p}$.

[11] Bock, R.D. and Petersen, A.C. (1975). A multivariate correction for attenuation. Biometrika, 62, 673-678.

[12] Bolfarine, H. and Zacks, S. (1992). Prediction Theory for Finite Populations, Springer-Verlag, NY, $205 \mathrm{p}$.

[13] Bouza, C.N. (1983). Estimation of a difference in finite populations with missing observations. Biometrical Journal, 25, 123-128.

[14] Box, G.E.P. and Tiao, G.C. (1968). Bayesian estimation of means for the random effects model. Journal of the American Statistical Association, 63, 174-181.

[15] Bussab, W.O. e Barroso, L.P. (1992). Painel Multivariado - Análise Através do Modelo de Componentes de Variância.. Instituto de Matemática e Estatística - USP, RT-MAE-9203.

[16] Calvin, J.A. (1993). REML estimation in unbalanced multivariate variance components models using an EM algorithm. Biometrics, 49, 691-701. 
[17] Chaubey, Y.P. (1983). A non-negative estimator of variance components closest to MINQUE. Sankhyã A, 45, 201-211.

[18] Chaubey, Y.P. (1984). On the comparison of some non-negative estimators of variance components for two models. Communications in Statistics B , 13, 619-633.

[19] Chauvenet, W. (1863). A Manual of Spherical and Practical Astronomy, 2: Theory and Use of Astronomical Instruments. Philadelphia, Lippincott.

[20] Chi, E.M. and Reinsel, G.C. (1989). Models for longitudinal data with random effects and AR(1) errors. Journal of the American Statistical Association, 84, 452459.

[21] Chiu, H.Y. and Sedransk, J. (1986). A bayesian procedure for imputing missing values in sample surveys. Journal of the American Statistics Association, 81, 667$676(*)$.

[22] Chow, S.C. and Shao, J. (1988). A new procedure for the estimation of variance components. Statistics and Probability Letters, 6, 349-355.

[23] Cochran, W.G. (1977). Sampling Techniques, Wiley, NY. 428 p.

[24] Cooper, L.G.; DeLeeuw, J. and Sogomonian, A.G. (1991). An imputation methods for dealing with missing data in regression. Applied Stochastic Models and Data Analysis, 7, 213-235 (*).

[25] Cornfield, J. and Tukey, J.W. (1956). Average values of mean squares in factorials. The Annals of Mathematical Statistics, 27, 907-949.

[26] Crump, S.L. (1947). The Estimation of Components of Variance in Multiple Classifications. Ph.D. Thesis, Iowa State University. 
[27] Crump, S.L. (1951). The present status of variance component analysis. Biometrics, $7,1-16$.

[28] Das, K. (1989). Estimation of variance components in an unbalanced two-way mixed model under heteroskedasticity. Journal of Statistical Planning and Inference, 21, 285-291.

[29] Das Peddada, S. (1989). Two non-negative estimators for the model with a common mean. Communications in Statistics B , 18, 501-512.

[30] Dempfle, L. (1977). Comparison of several sire evaluation methods in dairy cattle breeding. Livestock Prod. Science, 4, 129-139.

[31] Dempster, A.P.; Laird, N.M. and Rubin, D.B. (1977). Maximum likelihood estimation from incomplete data via the EM algorithm. Journal of the Royal Statistical Society - B , 39, 1-38.

[32] Duncan, G.J. and Kalton, G. (1987). Issues of design and analysis of surveys across time. International Statistics Review, 55, 97-117.

[33] Eisenhart, C. (1947). The assumptions underlying the analysis of variance. Biometrics, 3, 1-21.

[34] Elian, S.N. (1992). Análise de Regressão em Populações Finitas. Tese de Doutorado, Instituto de Matemática e Estatística - USP, São Paulo.

[35] Fellner, W.H. (1986). Robust estimation of variance components. Technometrics, 28, 51-60.

[36] Fellner, W.H. (1987). Sparse matrices and the estimation of variance components by likelihood methods. Communications in Statistics B , 16, 439-463. 
[37] Fisher, R.A. (1918). The correlation between relatives on the supposition of mendelian inheritance. Trans. R. Soc., Edinburgh., 52, 399-433.

[38] Gauss, K.F. (1809). Theoria motus corporum celestrium in sectionibus conics solem ambientium. Perthes and Besser: Hamburg.

[39] Gaylor, D.W. and Hartwell, T.D. (1969). Expected mean squares for nested classifications. Biometrics, 25, 427-430.

[40] Gilks, W.R.; Wang, C.C.; Yvonnet, B. and Coursaget, P. (1993). Random-effects models for longitudinal data using Gibbs sampling. Biometrics, 49, 441-453.

[41] Gnot, S. and Kleffe, J. (1983). Quadratic estimation in mixed linear models with two variance components. Journal of Statistical Planning and Inference, 8, 267-279.

[42] Goldberger, A.S. (1962). Best linear unbiased prediction in the generalized linear regression model. Journal of the American Statistical Association, 57, 369-375.

[43] Graybill, F.A. and Hultquist, R.A. (1961). Theorems concerning Eisenhart's model II. The Annals of Mathematical Statistics 32, 261-269.

[44] Grizzle, J.E. and Allen, D.M. (1969). Analysis of growth and dose response curves. Biometrics, 25, 357-381.

[45] Hartley, H.O. and Rao, J.N.K. (1967). Maximum likelihood estimation for the mixed analysis of variance model. Biometrika, 54, 93-108.

[46] Hartung, J. (1981). Nonnegative minimum biased invariant estimation in variance component models. The Annals of Statistics, 9, 278-292.

[47] Harville, D.A. (1974). Bayesian inference for variance components using only error contrasts. Biometrika 61, 383-385. 
[48] Harville, D.A. (1976). Extension of the Gauss-Markov theorem to include the estimation of random effects. The Annals of Statistics, 4, 384-395.

[49] Harville, D.A. (1977). Maximum likelihood approaches to variance component estimation and to related problems. Journal of the American Statistical Association, $72,320-340$.

[50] Harville, D.A. (1990). BLUP (Best Linear Unbiased Prediction) and beyond. Advances in Statistical Methods for Genetic Improvement of Livestock. D. Gianola and K. Hammond, eds. Springer, NY, 239-276.

[51] Harville, D.A. and Carriquiry, A.L. (1992). Classical and Bayesian prediction as applied to an unbalanced mixed linear model. Biometrics, 48, 987-1003.

[52] Heine, B. (1993). Nonnegative estimation of variance components in an unbalanced one way random effects model. Communications in Statistics A , 22, 2351-2372.

[53] Henderson, C.R. (1950). Estimation of genetic parameters. The Annals of Mathematical Statistics, 21, 309-310.

[54] Henderson, C.R. (1953). Estimation of variance and covariance components. Biometrics, 19, 226-252.

[55] Henderson, C.R. (1963). Selection index and expected genetic advance. Statistical Genetics and Plant Breeding. 141-163. Nat. Acad. Sci., Nat. Res. Council, Publication 982, Washington, D.C.

[56] Henderson, C.R. (1965). A method of determining if selection will bias estimates. Journal of Animal Science, 24, 849.

[57] Henderson, C.R. (1975). Best linear unbiased estimation and prediction under a selection model. Biometrics, 31, 423-447. 
[58] Hunt, D.N. and Triggs, C.M. (1989). Iterative missing value estimation. Applied Statistics, 38, 293-300(*).

[59] Ibrahim, J.G. (1990). Incomplete data in generalized linear models. Journal of the American Statistical Association, 85, 765-769.

[60] James, W. and Stein, C. (1961). Estimation with quadratic loss. Proc. Fourth Berkeley Symp. Math. Stat. Prob., 1, 361-379.

[61] Jarrett, R.G. (1978). The analysis of designed experiments with missing observations. Applied Statistics 27, 38-46.

[62] Jennrich, R.I. and Schluchter, M.D. (1986). Unbalanced repeated-measures models with structured covariance matrices. Biometrics, 42, 805-820.

[63] Jeske, D.R. and Harville, D.A. (1988). Prediction-interval procedures and (fixedeffects) confidence-interval procedures for mixed linear models. Communications in Statistics A, 17, 1053-1087.

[64] John, J.A. and Prescott, P. (1975). Estimating missing values in experiments. Applied Statistics, 24, 190-192 (*).

[65] Kackar, R.N. and Harville, D.A. (1981). Unbiasedness of two-stage estimation and prediction procedures for mixed linear models. Communications in Statistics A, 10, 1249-1261.

[66] Kackar, R.N. and Harville, D.A. (1984). Approximations for standard errors of estimators of fixed and random effects in mixed linear models. Journal of the American Statistical Association, 79, 853-862.

[67] Kale, B.K. and Chandrasekar, R. (1983). On the equivalence of optimality criteria for vector unbiased statistics. Journal of Indian Statistical Association, 21, 49-58. 
[68] Kalton,G. and Kasprzyk,D. (1982). Imputing for missing survey responses. Proceedings of the Survey Research Methods Section. American Statistical Association, 146-151 (*).

[69] Khatri, C.G. (1966). A note on a MANOVA model applied to problems in growth curve. Annals of the Institute of Statistical Mathematics, 18, 75-86.

[70] Khuri, A.I. and Sahai, H. (1985). Variance components analysis: a selective literature survey. International Statistics Review, 53, 279-300.

[71] Kish, L. (1965). Survey Sampling, Wiley, NY, 643 p.

[72] Kleinbaum, D.G. (1973). A generalization of the growth curve model which allows missing data. Journal of Multivariate Analysis, 3, 117-124.

[73] Klotz, J. (1990). Maximum likelihood estimators for the two way mixed model. Communications in Statistics A 19, 3741-3749.

[74] Lachenbruch, P.A. (1975). Discriminant Analysis. Hafner, New York.

[75] Laird, N.M. (1982). Computation of variance components using the EM algorithm. Journal of Statistical Computation and Simulation, 14, 295-303.

[76] Laird, N.M. (1988). Missing data in longitudinal studies. Statistics in Medicine, 7, 305-315.

[77] Laird, N.M.; Lange, N. and Stram, D. (1987). Maximum likelihood computations with repeated measures: application of the EM algorithm. Journal of the American Statistical Association, 82, 97-105.

[78] Laird, N.M. and Ware, J.H. (1982). Random-effects models for longitudinal data. Biometrics, 38, 963-974. 
[79] La Motte, L.R. (1970). A class of estimators of variance components. Technical Report 10, Department of Statistics, University of Kentucky, Lexington, Kentucky, 13 pages.

[80] La Motte, L.R. (1973a). Quadratic estimation of variance components. Biometrics, 29, 311-330.

[81] La Motte, L.R. (1973b). On non-negative quadratic unbiased estimation of variance components. Journal of the American Statistical Association, 68, 728-730.

[82] Lee, K.R. and Kapadia, C.H. (1992). Mean squared error efficient estimators of the variance components. Metrika 39, 21-26.

[83] Legendre, A.M. (1806). Nouvelles méthodes pour la determination des orbites des cométes; avec un supplément contenant divers perfectionnements de ces méthodes et leur application aux deux cométes de 1805. Courcier, Paris.

[84] Liang, K.Y. and Zeger, S.L. (1986). Longitudinal data analysis using generalized linear models. Biometrika 73, 13-22.

[85] Lindley, D.V. and Smith, A.F.M. (1972). Bayes estimates for the linear model (with discussion). Journal of the Royal Statistical Society B, 34, 1-41.

[86] Lindstrom, M.J. and Bates, D.M. (1988). Newton-Raphson and EM algorithms for linear mixed-effects models for repeated-measures data. Journal of the American Statistical Association, 83, 1014-1022.

[87] Lindstrom, M.J. and Bates, D.M. (1990). Nonlinear mixed effects models for repeated measures data. Biometrics 46, 673-687.

[88] Liski, E.P. (1985). Estimation from incomplete data in growth curves models. Communications in Statistics B 14, 13-27. 
[89] Little, R.J.A. (1988). Comment on analysis of data with missing values. Statistics in Medicine, 7, 347-355.

[90] Little, R.J.A. and Rubin, D.B. (1983). Missing data in large data sets. Statistical methods and the improvement of data quality, Tommy Wright, Academic, NY, 215-243.

[91] Little, R.J.A. and Rubin, D.B. (1987). Statistical Analysis with Missing Data, Wiley, NY, $278 \mathrm{p}(*)$.

[92] Little, R.J.A. and Smith, P.J. (1987). Editing and imputation for quantitative survey data. Journal of the American Statistical Association, 82, 58-68 (*).

[93] Magnus, J.R. and Neudecker, H. (1988). Matrix Differential Calculus with Applications in Statistics and Econometrics, John Wiley \& Sons, 393 p.

[94] Mardia, K.V.; Kent, J.T. and Bibby, J.M. (1979). Multivariate Analysis, Academic Press, NY, 518 p.

[95] Mathew, T.; Sinha, B.K. and Sutradhar, B.C. (1992). Nonnegative estimation of variance components in unbalanced mixed models with two variance components. Journal of Multivariate Analysis, 42, 77-101.

[96] McGilchrist, C.A. (1994). Estimation in generalized mixed models. Journal of the Royal Statistical Society B, 56, 61-69.

[97] Mensah, R.D.; Elswick Jr., R.K. and Chinchilli, V.M. (1993). Consistent estimators of the variance-covariance matrix of the GMANOVA model with missing data. Communications in Statistics A, 22, 1495-1514.

[98] Park, T, and Woolson, R.F. (1992). Generalized multivariate models for longitudinal data. Communications in Statistics B, 21, 925-946. 
[99] Patterson, H.D. and Thompson, R. (1971). Recovery of inter-block information when block sizes are unequal. Biometrika, 58, 545-554.

[100] Patterson, H.D. and Thompson, R. (1975). Maximum likelihood estimation of components of variance. Proceedings of the 8th International Biometric Conference, 197-207.

[101] Peres, C.A. and Saldiva, C.D. (1982). Planejamento de Experimentos, 5o SINAPESimpósio Nacional de Probabilidade e Estatística - Associação Brasileira de Estatística, $98 \mathrm{p}$.

[102] Potthoff, R.F. and Roy, S.N. (1964). A generalized multivariate analysis of variance model useful specially for growth curve problems. Biometrika, 51, 313-326.

[103] Rao, C.R. (1965). The theory of least squares when the parameters are stochastic and its application to the analysis of growth curves. Biometrika, 52, 447-458.

[104] Rao, C.R. (1967). Least squares theory using and estimated dispersion matrix and its application to measurement of signals. Proceedings of the Fifth Berkeley Symposium on Mathem. Statist. Probab. Vol. I, Berkeley and Los Angeles: University of California Press. 355-372.

[105] Rao, C.R. (1970). Estimation of heteroscedastic variances in a linear model. Journal of the American Statistical Association 65, 161-172.

[106] Rao, C.R. (1971a). Estimation of variance and covariance components - MINQUE Theory. Journal of Multivariate Analysis 1, 257-275.

[107] Rao, C.R. (1971b). Minimum variance quadratic unbiased estimation of variance components. Journal of Multivariate Analysis, 1, 445-456.

[108] Rao, C.R. (1972). Estimation of variance and covariance components in linear models. Journal of the American Statistical Association, 67, 112-115. 
[109] Rao, C.R. (1973). Linear Statistical Inference and Its Applications, Wiley, NY - $2^{\underline{a}}$ edição, 625 p.

[110] Rao, C.R. (1979). MINQUE-theory and it relation to ML and MML estimation of variance components. Sankhyã B 41, 138-153.

[111] Rao, C.R. (1987). Prediction of future observations in growth curve models. Statistical Science, 2, 434-471 (*).

[112] Rao, C.R. and Kleffe, J. (1988). Estimation of Variance Components and Applications, North-Holland, Amsterdam, $370 \mathrm{p}$.

[113] Rao, J.N.K. and Subrahmaniam, K. (1971). Combining independent estimators and estimation in linear regression with unequal variances. Biometrics, 27, 971-990.

[114] Rao, P.S.R.S. and Chaubey, Y.P. (1978). Three modifications of the principle of MINQUE. Communications in Statistics A , 7, 767-778.

[115] Rao, P.S.R.S.; Kaplan, J. and Cochran, W.G. (1981). Estimators for the one-way random-effects model with unequal error variances. Journal of the American Statistical Association, 76, 89-97.

[116] Reinsel, G. (1982). Multivariate repeated-measurement or growth curve models with multivariate random-effects covariance estructure. Journal of the American Statistical Association, 77, 190-195.

[117] Reinsel, G. (1984). Estimation and prediction in a multivariate random effects generalized linear model. Journal of the American Statistical Association, 79, 406-414 $(*)$.

[118] Reinsel, G.C. (1985). Mean squared error properties of empirical bayes estimators in a multivariate random effects general linear model. Journal of the American Statistical Association, 80, 642-650. 
[119] Robinson, D.L. (1987). Estimation and use of variance components. The Statistician, 36, 3-14.

[120] Robinson, G.K. (1991). That BLUP is a good thing: the estimation of random effects. Statistical Science, 6, 15-51.

[121] Rubin, D.B. (1976). Inference and missing data. Biometrika, 63, 581-592.

[122] Rubin, D.B. (1987). Multiple Imputation for Nonresponse in Surveys, Wiley, NY, $258 \mathrm{p}(*)$.

[123] Sahai, H.; Khuri, A.I. and Kapadia, C.H. (1985). A second bibliography on variance components. Communications in Statistics A, 14, 63-115.

[124] Schall, R. (1991). Estimation in generalized linear models with random effects. Biometrika, 78, 719-727.

[125] Scheffé, H. (1956). Alternative models for the analysis of variance. The Annals of Mathematical Statistics, 27, 251-271.

[126] Schluchter, M.D. (1988). Analysis on incomplete multivariate data using linear models with structured covariance matrices. Statistics in Medicine, 7, 317-324.

[127] Searle, S.R. (1971). Linear Models, John Wiley \& Sons, NY, 532 p.

[128] Searle, S.R. (1987). Linear Models for Unbalanced Data, John Wiley \& Sons, NY, $536 \mathrm{p}$.

[129] Searle, S.R. (1988). Mixed models and unbalanced data: wherefrom, whereat and whereto? Communications in Statistics A, 17, 935-968. 
[130] Searle, S.R.; Casella, G. and McCulloch, C.E. (1992). Variance Components, Wiley, NY, $501 \mathrm{p}$.

[131] Searle, S.R. and Fawcett, R.F. (1970). Expected mean squares in variance components models having finite populations. Biometrics, 26, 243-254.

[132] Silva, P.L.N. (1989). Crítica e Imputação de Dados Quantitativos Utilizando o SAS. Dissertação de Mestrado, IMPA-RJ (*).

[133] Singh, R. (1985). Estimation from incomplete data in longitudinal surveys. Journal of Statistical Planning and Inference 11, 163-170 (*).

[134] Singh, S.P. and Pratap, M. (1989). A non-iterative method for least squares estimation of missing values in any design. Journal of the Indian Statistical Association 27, 63-71(*).

[135] Speed, T. (1991). Comment on Robinson: estimation of random effects. Statistical Science, 6, 42-44.

[136] Srivastava, M.S. (1985). Multivariate data with missing observations. Communications in Statistics A 14, 775-792.

[137] Stiratelli, R.; Laird, N. and Ware, J.H. (1984). Random-effects models for serial observations with binary response. Biometrics, 40, 961-971.

[138] Stuart, A. and Ord, J.K. (1987). Kendal's Advanced Theory of Statistics - vol. 1, Charles Griffin \& Company Limited, London, 604 p.

[139] Subramani, J. (1991). On invariant quadratic unbiased estimation of variance components. Communications in Statistics A, 20, 1705-1730. 
[140] Subramani, J. (1992). Analysis of means for experimental designs with missing • observations. Communications in Statistics A, 21, 2045-2057.

[141] Subramani, J. and Ponnuswamy, K.N. (1989). A non-iterative least square estimation of missing value in experimental designs. Journal of Applied Statistics, 16, $77-86(*)$.

[142] Tam, S.M. (1986). Optimal prediction in stochastic regression models with application to the analysis of repeated surveys. The Australian Journal of Statistics, 28, 345-353.

[143] Thompson, R. (1980). Maximum likelihood estimation of variance components. Statistics, 11, 545-561.

[144] Thompson Jr., W.A. (1962). The problem of negative estimates of variance components. The Annals of Mathematical Statistics, 33, 273-289.

[145] Tukey, J.W. (1956a). Keeping moment-like sampling computations simple. The Annals of Mathematical Statistics, 27, 37-54.

[146] Tukey, J.W. (1956b). Variances of variance components: I: balanced designs. The Annals of Mathematical Statistics, 27, 722-736.

[147] Tukey, J.W. (1957a). Variances of variance components: II: unbalanced single classifications. The Annals of Mathematical Statistics, 28, 43-56.

[148] Tukey, J.W. (1957b). Variances of variance components: III: third moments in a balanced single classification. The Annals of Mathematical Statistics, 28, 378-384.

[149] Volaufová, J. and Witkovsky, V. (1992). Estimation of variance components in mixed linear models. Applications of Mathematics, 37, 139-148. 
[150] Wang, C.M. (1983). On the analysis of multivariate repeated measures designs. Communications in Statistics A, 12, 1647-1659.

[151] Wang, R.; Sedransk, J. and Jinn, J.H. (1992). Secondary data analysis when there are missing observations. Journal of the American Statistical Association, 87, 952$961(*)$.

[152] Wansbeek, T. and Kapteyn, A. (1989). Estimation of the error-components model with incomplete panels. Journal of Econometrics, 41, 341-361.

[153] Ware, J.H. (1985). Linear models for the analysis of longitudinal studies. The American Statistician, 39, 95-101.

[154] Wesolowska, M.T. and Janczarek (1984). Estimation of covariance matrices in unbalanced random and mixed multivariate models. Biometrical Journal, 26, 665-674.

[155] Wilkinson, G.N. (1958a). Estimation of missing values for the analysis of incomplete data. Biometrics 14, 257-286 (*).

[156] Wilkinson, G.N. (1958b). The analysis of variance and derivation of standard errors for incomplete data. Biometrics, 14, 360-384.

[157] Winer, B.J. (1971). Statistical Principles in Experimental Design, 2a edição, McGraw-Hill, NY, 907 p.

[158] Wishart, J. (1952). Moment coefficients of the k-statistics in samples from a finite population. Biometrika, 39, 1-13.

[159] Wolfram, S. (1991). Mathematica: A System for Doing Mathematics by Computer, $2^{\underline{a}}$ edição, Addison-Westey, California, 961 p. 
[160] Wu, M.C. and Bailey, K. (1988). Analysing changes in the presence of informative right censoring. Statistics in Medicine, 7, 337-346.

[161] Yates, F. (1933). The analysis of replicated experiments when the field results are incomplete. Empire Journal of Experimental Agriculture, 1, 129-142 (*).

[162] Zeger, S.L.; Liang, K.Y. and Albert, P.S. (1988). Models for longitudinal data: a generalized estimating equation approach. Biometrics, 44, 1049-1060. 